



\title{
Pulsed Second Order Magnetic Field Nuclear Magnetic Resonance for Real-Time Fluid Transport Measurements
}

\author{
by
}

Wilfred Kittler

A thesis

submitted to the Victoria University of Wellington in fulfilment of the requirements for the degree of

Doctor of Philosophy

Victoria University of Wellington

2014 



\section{Abstract}

Nuclear magnetic resonance (NMR) measurement techniques are able to characterise a large array of physical properties and systems, both transparent and opaque, in a nondestructive, non-invasive manner. This allows systems to be probed with minimal disturbance, and in most cases, information can be obtained with little to no experimental bias. For these reasons NMR lends itself well to many fields of research. Of particular importance to this research is NMR measurement of the characteristic function which defines fluid transport known as the average propagator. This quantity provides the probability distribution for fluid displacement, and all mobility information of the fluid matter under measurement.

The average propagator is normally measured by NMR with a series of experiments which require an extended period of time. When systems are evolving on a time scale which is shorter than the total experimental time required to measure the average propagator, the measurement cannot be performed as different experiments in the series relate to different states of the system.

In this thesis a method for transforming the slow, serial process of measuring the average propagator into an instantaneous, parallel process has been developed. This allows realtime characterisation of flows, diffusion, and the properties of the pore spaces in porous media containing fluid. The details of the newly developed technique are provided along with new hardware designed and built to perform the new parallel method, allowing instantaneous measurement of the average propagator.

Experimental results are presented for well known model systems. These systems are used because their properties and models describing their behaviour are well understood. The experimental measurements for these model systems were compared to theoretical predic- 
tions to verify the effectiveness of the new average propagator measurement technique, providing a proof of concept, and proving the validity of this new average propagator measurement for real-time characterisation of fluid transport and porous media. 


\section{Acknowledgements}

Many people have given me great deal of support in this process, and in this brief acknowledgement I will attempt to do them justice.

The first person I should thank, not for his support in performing the research contained in this thesis, but for the many opportunities, support, and confidence he gave me, is the late Professor Sir Paul Callaghan. For the brief time I knew him it was always a pleasure to be in his presence, which was certainly in high demand. When he spoke, people listened, and for good reason. I was always hoping some of his brilliance might rub off on me. He was a man who brought out the best in people. He gave me confidence, was, and is still a source of inspiration. Paul also played an important role in bringing me to New Zealand where I met my wife Jane, and made certain I would be able to support my family throughout this process. For all of these things, Paul, I thank you.

My primary supervisor Mark Hunter was the driving force behind this research. In fact before I was studying as a PhD student, Mark was also my supervisor at Magritek Ltd. Mark, I would like to thank you for all your help, especially at the beginning of my study. Mark used to sit with me at the spectrometer explaining the intricacies of NMR, helping me come to grasp with the tasks ahead of me. He has been a dedicated supervisor, a skilled experimentalist, and has kept me on track, always with the next step of this research in mind.

Petrik Galvosas has gone above and beyond his role as my secondary supervisor, providing the guidance for publication, helping me understand how to present and write to the scientific community. He has also asked me some of the deeper questions about this research, inspiring me to develop theory and gain a better understanding. Although perpetually busy, Petrik has always been there with an open door willing to listen and 
help.

I would also like to express my gratitude to the entire physics NMR lab at the Victoria University of Wellington. Everyone from when I began designing electromagnetic coils for Magritek Ltd. in 2009, all the way through completion of this thesis in 2014, thank you for your help.

To my mother, father, and sister, thank you for your support throughout my life. You have always had confidence in me, even when I lacked confidence in myself. I miss you all, and although you are all the way across the Pacific, you have been a source of strength throughout.

To all my children: Taine, Grayson, Ava, Ryder, Taylor, and Chase, thank you for keeping home life action packed, I wouldn't have it any other way. You keep me balanced, and have helped me become a better person. Although none of you quite understand what I do, your faith in me has helped me persevere.

Of course the most important acknowledgement of all goes to my beautiful wife Jane. With a vast lack of experience in science and absolutely no knowledge of physics, after listening to me, you somehow always knew exactly what I should do. By far the best piece of advice was when you told me to start writing this thesis far ahead of time. You have endured this process along side me with the grace that only you possess. You have given me two beautiful children during this research, and while studying and working yourself, kept the family and house ticking over with clockwork precision, something I could not do even if I had all the time in the world. I do not know where you find your energy or patience, but without you this would not have been possible. 


\section{Contents}

1 Introduction 1

2 Fluid Transport in Porous Media $\quad 7$

2.1 Introduction . . . . . . . . . . . . . . . . . . . 7

2.2 Diffusion . . . . . . . . . . . . . . . . . . . . . . . . . . . . 8

2.2 .1 Introduction . . . . . . . . . . . . . . 8

2.2 .2 Brownian Motion . . . . . . . . . . . . . . . . . 8

2.2 .3 Fick's Laws . . . . . . . . . . . . . . . . . . . . . . . . . . 9

2.3 The Propagator . . . . . . . . . . . . . . . . . . . . 10

2.3.1 Probability Density and Fick's Laws . . . . . . . . . . . . . . 10

2.3.2 The Average Propagator . . . . . . . . . . . . . . . . . . . . 12

2.3.3 The Average Propagator for Pipe Flow . . . . . . . . . . . . . . . 13

2.3.4 The Propagator for Restricted Diffusion . . . . . . . . . . . . . . 16

2.4 Porous Media . . . . . . . . . . . . . . . . . . . . . . . . . . . . . . . 18

2.4.1 Definition of Porous Media . . . . . . . . . . . . . . . 18

2.4.2 Properties of Porous Media . . . . . . . . . . . . . . . . . . . . 19

2.5 Conclusions . . . . . . . . . . . . . . . . . . . 24

3 Nuclear Magnetic Resonance $\quad 25$

3.1 The NMR Signal . . . . . . . . . . . . . . . . . . . . . . . 25

3.1.1 Introduction . . . . . . . . . . . . . . . . 25

3.1 .2 Nuclear Spin . . . . . . . . . . . . . . . . . 25

3.1.3 Polarisation . . . . . . . . . . . . . . . . . . . 27 
3.1.4 Excitation . . . . . . . . . . . . . . . . . . 28

3.2 Relaxation Effects . . . . . . . . . . . . . . . . . . . . . . 34

3.2.1 The FID and $T_{2^{*}}$ Relaxation . . . . . . . . . . . . . 34

$3.2 .2 T_{2}$ Relaxation . . . . . . . . . . . . . . . . . . 39

$3.2 .3 T_{1}$ Relaxation . . . . . . . . . . . . . . . . . . 40

3.2.4 Bloch Equations . . . . . . . . . . . . . . . . . 44

3.3 Conclusions . . . . . . . . . . . . . . . . . . . . . 45

4 Pulsed Magnetic Field Gradients in NMR 47

4.1 Introduction . . . . . . . . . . . . . . . . . . . . . . 47

4.2 Constant Magnetic Field Gradients . . . . . . . . . . . . . . . 47

4.3 Spatial Resolution With Constant Magnetic Field Gradients . . . . . . . 50

4.3.1 $k$-Space and the Read Gradient . . . . . . . . . . . . . 50

4.3 .2 Slice Selection . . . . . . . . . . . . . . . . . 55

4.4 Measuring Displacement With Constant Magnetic Field Gradients . . . . 58

4.4.1 The PGSE Experiment \& q-Space . . . . . . . . . . . . . . . 58

4.4.2 Measuring Diffusion with PGSE . . . . . . . . . . . . . . 59

4.4.3 Shaped Gradient Pulses in PGSE Diffusion Measurement . . . . . 65

4.4.4 Measuring the Propagator with PGSE . . . . . . . . . . . . 67

4.4.5 The Stimulated Echo and Pulsed Gradient Stimulated Echo . . . 68

4.4.6 Difftrain . . . . . . . . . . . . . . . . . . . . 72

4.5 Conclusions . . . . . . . . . . . . . . . . . . . . 74

5 Pulsed Second Order Field NMR $\quad 75$

5.1 Introduction . . . . . . . . . . . . . . . . . . 75

5.2 Non-Linear Magnetic Fields . . . . . . . . . . . . . . . . . 75

5.2.1 Effects and Uses of Non-Linear Magnetic Fields in NMR . . . . . 75

5.2.2 Second Order Magnetic Fields . . . . . . . . . . . . . . . 77

5.3 Second Order Field PGSE for Diffusion Measurements . . . . . . . . . . 78

5.3.1 The Basic Pulsed Second Order Field Sequence for Parallel Acquisition of $q$-Space . . . . . . . . . . . . . . . . . . . 78 
5.3.2 Independence of Diffusion Measurements on Slice Thickness . . . 81

5.3.3 Validity of Diffusion Measurements Using $2^{\text {nd }}$ Order Fields . . . . 83

5.3.4 Multiple Observation Time Diffusion Measurements with a Second Order Field in Difftrain . . . . . . . . . . . . . . . 86

5.3.5 Incorporation of a 13-Interval Pulse Sequence in the Modified Difftrain Method . . . . . . . . . . . . . . . . 88

5.4 Time domain signal as the average propagator . . . . . . . . . . . . 90

5.4 .1 Introduction . . . . . . . . . . . . . . . . . . . . 90

5.4.2 Comparison to other displacement measurements . . . . . . . . . 90

5.4 .3 Single-shot propagator measurement . . . . . . . . . . . . 91

5.4.4 Long displacement and strong curvature limit . . . . . . . . . . . 94

5.4 .5 New echo condition . . . . . . . . . . . . . . . . . . . . 95

5.4.6 Phase Evolution Echo Derivation . . . . . . . . . . . . 96

5.4.7 Complex time to real displacement mapping through phase evolution 100

5.4.8 Conditions for the echo as the average propagator . . . . . . . . . 101

$5.4 .9 T_{2}^{*}$ Considerations . . . . . . . . . . . . . . . 103

5.5 Conclusions . . . . . . . . . . . . . . . . . . . . 104

6 Coil Fabrication \& Characterisation 105

6.1 Introduction . . . . . . . . . . . . . . . . . . . . 105

6.2 Design Considerations for Magnetic Field Gradient Coils . . . . . . . . . 106

6.2.1 Introduction to Design Considerations . . . . . . . . . . . . 106

6.2.2 Maxwell's Equations and Magnetic Field Gradients . . . . . . . . 109

6.3 Coil Design for Halbach Geometry . . . . . . . . . . . . . . . . . . . . . . 111

6.3.1 Design Parameters . . . . . . . . . . . . . . . . 111

6.3.2 First Order Field Requirements . . . . . . . . . . . . . . . . . . . 113

6.3.3 Second Order Field Requirements . . . . . . . . . . . . . . . . 116

6.3.4 Modelling Gradient Coil Characteristics . . . . . . . . . . . . . 117

6.3.5 Fabrication \& Mapping . . . . . . . . . . . . . . . . . . . . 124

6.4 Coil Design for Superconducting Geometry . . . . . . . . . . . . . . . . . 130

6.4.1 Design Restrictions . . . . . . . . . . . . . . . . 130 
6.4.2 Fabrication . . . . . . . . . . . . . . . . . . 132

6.5 Conclusions . . . . . . . . . . . . . . . . . . . . . . . . . 132

7 Experimental Results $\quad 133$

7.1 Introduction . . . . . . . . . . . . . . . . . . . 133

7.2 Low Field Parallel Acquisition of $q$-Space \& Single-Shot Diffusion Mea-

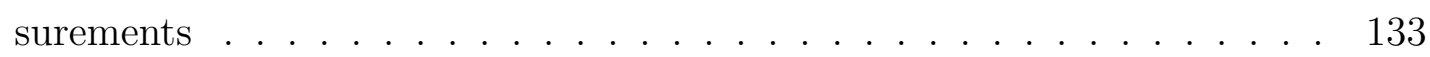

7.2 .1 Introduction . . . . . . . . . . . . . . . . . . . 133

7.2.2 Diffusion Measurements with Slice Selection . . . . . . . . . . . . 134

7.2.3 Echo as the Diffusion Propagator . . . . . . . . . . . . . . . . . . 138

7.2.4 Diffusion Measurements Without Slice Selection . . . . . . . . . . 139

7.3 Low Field Diffusion Measurements Using the Modified Difftrain Pulse Sequence . . . . . . . . . . . . . . . . . . . . . . . . 142

7.3 .1 Introduction . . . . . . . . . . . . . . . . . . . . . . . 142

7.3.2 Samples . . . . . . . . . . . . . . . . . . . . . . 142

7.3.3 Free Diffusion Measurements . . . . . . . . . . . . . . . . . . . . . 143

7.3.4 Restricted Diffusion Measurements Spanning the Short and Long Time Limits . . . . . . . . . . . . . . . . . . . . . . . . . . . 145

7.3.5 Short Time Limit Restricted Diffusion Measurements . . . . . . . 148

7.4 High Field Measurements Using the Modified Difftrain Pulse Sequence . 150

7.4 .1 Introduction . . . . . . . . . . . . . . . . . 150

7.4 .2 Sample Characterisation . . . . . . . . . . . . . . 151

7.4.3 Short Time Limit Restricted Diffusion Measurements . . . . . . . 151

7.5 High Field Single-Shot Propagator Measurements . . . . . . . . . . . . . 154

7.5.1 Introduction . . . . . . . . . . . . . . . . . . . . . 154

7.5.2 Hardware and Experimental Set-Up . . . . . . . . . . . . . . . . 154

7.5.3 Verifying Experimental Requirements for the Echo as the Propagator 155

7.5.4 Propagator Measurements for Ramped Flow . . . . . . . . . . . . 158

7.5.5 Complex Mapping for Large Displacements . . . . . . . . . . . . . 161 
8.1 Results Presented in this Thesis . . . . . . . . . . . . . . . . . 165

8.1.1 Parallel Acquisition of $q$-Space . . . . . . . . . . . . . . . . 165

8.1.2 Diffusion Measurements . . . . . . . . . . . . . . 166

8.1.3 Propagator Measurements . . . . . . . . . . . . . . 167

8.2 Future Work . . . . . . . . . . . . . . . . . . . . 167

8.2.1 Different Samples and Systems . . . . . . . . . . . . . . . 167

8.2.2 Different Methods and Techniques . . . . . . . . . . . . . . 168

8.3 Final Thoughts . . . . . . . . . . . . . . . . . . . . . . 169 



\section{Chapter 1}

\section{Introduction}

Nuclear magnetic resonance is a widely used technique to non-invasively measure the physical properties and characteristics of materials, living organisms, and systems in general. Its success in numerous fields is due largely to its ability to extract information from opaque systems with minimal disturbance, allowing the systems to continue functioning as if nothing had occurred. This passive probing lends itself well to the characterisation of fluid transport and porous media where data can be obtained from a complex network in situ.

Porous media encompasses many materials and systems around us. They range from the human body whose internal transport properties describe our vasculature and neural network down to rocks from which the oil and gas industry drill into and pump from. Predicting and modelling the behaviours of such systems allows calculated and intelligent intervention to alter their performance and acquire a desired outcome whether it be inhibiting neurons from firing to prevent seizures, or increasing the yield of an oil well. The extraction of diffusion, dispersion, and flow information without biasing the measurement by use of NMR techniques greatly improves this ability.

Measurement of the probability distribution for displacement, i.e. the average propagator, provides a method of characterising the mobility of particles in fluid matter. When fluid resides in the pore space of a porous medium, the average propagator provides information about the pore space. As the pore boundaries of the pore space present obstacles 
for displacement, they influence diffusive behaviour, flow, and diffusion within a flow field (dispersion). This biased movement can then be used to extract properties such as pore size, shape, interconnectivity, tortuosity, surface to volume ratio, and permeability [1-34]. When these properties are changing in time, displacement information not only provides a way to characterise the medium, but also information about how the system is evolving.

The characterisation of fluid transport is also of interest to a wide array of basic research and industrial applications. Transient flows through complex networks, pulsed flow, transient effects in non-Newtonian fluids, and start-up or unstable flow fields are of interest to a wealth of processes in engineering and science [7, 25, 35]. Computational fluid dynamics provide numerical simulations which can model such processes [36-39], and velocity imaging methods can rapidly provide maps of the mean displacement per voxel [40-46], but to obtain the full distribution of displacements the average propagator is required. The pulsed gradient spin echo (PGSE) NMR experiment [47] is the conventional technique used to measure the average propagator and to characterise porous media [10$14,20,32,33,41,48-55]$. To obtain the necessary data set for the average propagator, this method requires a series of experiments where the total experimental time is limited by $T_{1}$ relaxation of the nuclei between experiments. The time constant $T_{1}$ determines the time required for the system to return to thermal equilibrium such that another experiment can be performed. For hydrogen in water molecules, the primary nuclei used for NMR, this relaxation is on the order of a few seconds. Normally, five times the time constant $T_{1}$ represents the repetition time between experiments, making the temporal resolution for displacement measurements using conventional PGSE, at minimum, on the order of seconds. If a system is evolving on a time scale faster than this, the data acquired from the series of experiments will relate to different states of the system, and the data set will not provide accurate displacement information.

Traditionally, first order magnetic fields which possess a linear relationship between field strength and position, and therefore a constant magnetic field gradient strength, are pulsed to encode for displacement. To acquire displacement information, a series of experiments are required where the strength of the gradient is varied between experiments. In this research, pulsed second order magnetic fields whose field strength varies as the 
square of two axes are used to encode for displacement. The gradient strength of such a field varies in space, allowing a range of gradients to be applied across the sample in a single experiment. Along with imaging methods, this allows displacement information, and the average propagator, to be obtained in real-time using a single experiment for samples which are homogeneous on a length scale of millimetres or smaller.

Second order magnetic field coils were built for these experimental purposes, characterised with a variety of techniques, and used to implement the new experimental methods. These methods were applied to perform real-time measurements of diffusion coefficients, surface to volume ratios of bead packs of spherical beads, and the average propagator for laminar flows of water through a cylindrical pipe. Well defined systems were chosen for these experiments such that the experimental results could be compared against theoretical predictions, and in all cases were found to be in excellent agreement. These results provide a proof of concept for these new methods of real-time displacement measurements for characterisation of porous media and flows, as well as its viability for use in characterising and monitoring quickly evolving systems which are not yet well defined.

\section{Chapter 2}

Here the phenomenon of self-diffusion is introduced. An explanation of how the diffusive process becomes restricted when particles reside in a porous medium, and how measurement of the apparent diffusion coefficient provides information about the porous medium is described. The probability distribution of displacements, i.e. the average propagator, is introduced, including its relevance in restricted diffusion and characterising flow. Lastly, a definition of porous media is presented along with its defining characteristics.

\section{Chapter 3}

This chapter provides the basic theoretical framework behind the phenomenon known as nuclear magnetic resonance (NMR). Quantum and semi-classical behaviour is considered, and the basic methods through which the NMR signal is generated and decays are 
explained.

\section{Chapter 4}

The pulsing of magnetic fields whose strength varies in space are introduced in this chapter, along with their applications towards measurement techniques in NMR. The theoretical considerations for their use in spatial and displacement encoding are discussed. The theory of spin evolution in the presence of these pulsed fields is described along with the basic pulse sequences used to perform the various encodings and resulting measurements relevant to this research.

\section{Chapter 5}

This chapter builds on all previous chapters, and is the beginning of the original research presented in this thesis. The second order magnetic field used in this research is introduced along with its effects in displacement encoding for NMR measurements. The previously described measurement techniques are combined and modified to propose new parallel measurement techniques, with theoretical and experimental considerations addressed.

\section{Chapter 6}

In this chapter the process of creating the necessary new hardware to enable the proposed measurement techniques is discussed. Specifications for the required hardware are carefully defined along with theoretical modelling of proposed designs, fabrication processes, and characterisation of the custom hardware. 


\section{Chapter 7}

In the experimental results chapter, all proposed measurement techniques are implemented, and results compared against theoretically predictions. Experimental limitations and justification for experimental parameters are discussed. This provides a proof of concept for the real-time acquisition of displacement data for diffusion, surface to volume ratio, and average propagator measurements proposed in chapter 5.

\section{Chapter 8}

Here the experimental results are reflected upon, with a summary and brief analysis of the findings. Modifications to the presented techniques as well as incorporation with other NMR measurements are proposed. Finally, proposed applications of this rapid measurement technique to enable characterisation of systems in which processes are varying on the sub-second time scale are provided. 



\section{Chapter 2}

\section{Fluid Transport in Porous Media}

\section{$2.1 \quad$ Introduction}

Porous media is a broad field which encompasses the majority of materials on this planet. With knowledge of their properties and function, intelligent and planned interaction with them is more likely to result in a desired outcome.

The pore boundaries will influence the diffusion, flow, and dispersion of fluids in the pore space. Through measurement of the average propagator, the probability distribution of particle displacement in time, fluid transport can be characterised. When the fluid resides in the pore space of a porous medium, the pore space boundaries influence its transport, and information is also obtained about the structure of the pore space. This information allows the determination of attributes such as porosity, tortuosity, pore size, pore shape, the surface to volume ratio, and flow fields.

In this chapter a brief overview of diffusion and porous media is given, with detailed descriptions of flow in porous media found elsewhere in standard texts [56, 57]. 


\subsection{Diffusion}

\subsubsection{Introduction}

The theory of diffusion stems back to statistical mechanics and the notion of atoms and molecules. The thermal energies associated with these species causing random motions and displacement, analysed statistically, gives rise to time dependence in concentration gradients, and the concept of self diffusion. In this chapter, an overview is provided of the development of the theory behind diffusion, particularly self-diffusion, the ways in which its behaviour is defined and characterised, and its implications towards NMR measurements.

\subsubsection{Brownian Motion}

One of the first well documented observations of a diffusive process was noted by the botanist Robert Brown [58] in 1827 when studying the movements of pollen grains suspended in water. He noted these pollen grains seemed to move in a random fashion and could not specify the forces acting upon them as he was convinced they were not due to currents or evaporative processes. In 1905, Albert Einstein published a paper which offered a detailed description of the processes at play in Brown's observations which accurately predicted the movements of these pollen grains as being caused by the random movements of the water molecules in which they were suspended [59]. This notion of atoms and molecules had been present for some time with a lack of evidence, but had been postulated and previously used to describe phenomena by Bernoulli, and even derive the laws of thermal dynamics by Boltzmann.

Although the concept of molecules and atoms had not been proven experimentally, Avagadro used this notion to relate the volume of a gas at given temperature and pressure to the number of atoms or molecules present, Fick to describe behaviour of solutes in a concentration gradient, and Bernoulli to describe gas pressure through the forces caused by collisions between gas species and walls. Boltzmann had postulated that for every type of motion, there would be a proportional thermal energy partitioned equally among such motions, namely, the concept of equipartition. Using Bernoulli's ideas on gas pressure, 
he combined the concept of equipartition to that of atoms and molecules, and was able to derive the gas laws. Not only did he provide an explanation of gas properties, but was also able to derive the laws of thermodynamics using statistics for large numbered systems, an acceptable approach when dealing with these small constituents.

In early 1900's, Einstein described the random movement of Brown's pollen grains through the use of Boltzmann's ideas on thermal energy. Einstein claimed the pollen grains were small enough to be influenced by the random motions of the water molecules in which they were suspended, and provided a rule relating Avagadro's number to the displacement in time of the pollen grain. Through optical measurements, a physicist by the the name of Jean Perrin experimentally verified this, writing a number of papers concerning Brownian motion and molecular reality [60-63], and subsequently won the nobel prize by determination of Avagadro's number by this and other techniques.

\subsubsection{Fick's Laws}

Predating the verification of atoms and molecules, in 1855, Adolf Fick presented a description of diffusion to explain the observations of a non-uniform solute concentration in time [64]. This analysis allowed for a continuous distribution of concentration, and from this analysis stemmed two laws which are described in the following.

Fick described the diffusive process using a function representing the concentration of a solute, or particle concentration $n(\mathbf{r}, t)$, which varies in space. Fick's first law relates the concentration gradient to the flux of the particles $\mathbf{J}$, through a diffusion coefficient $D$.

$$
\mathbf{J}=-D \nabla n(\mathbf{r}, t)
$$

Fick's second law is a consequence of conservation of the solute, which requires the time rate of change of the local solute concentration to equal the negative of the particle flux divergence.

$$
-\nabla \cdot \mathbf{J}=\frac{\partial n}{\partial t}
$$


Substituting Eq. 2.2 into Eq. 2.1 yields Fick's second law, also know as the diffusion equation.

$$
\frac{\partial n}{\partial t}=D \nabla^{2} n
$$

As previously stated, Fick's derivation was aimed at describing known processes pertaining to solute gradients. As time evolved, these concentration gradients were known to disappear to produce a uniform solution. This was before the work of Boltzmann, who paved the way for thermodynamics. Einstien was able to take Fick's thoughts on diffusion and employ Boltzmann's work on thermal energies to take diffusion a step further, explaining self-diffusion, the diffusive process in the absence of concentration gradients.

\subsection{The Propagator}

\subsubsection{Probability Density and Fick's Laws}

Similar to the concentration function, Einstein was able to show that the probability density also satisfied Fick's laws. This is of great importance as the propagator, which provides the likelihood of finding a particle which has undergone some displacement in time, will be defined by a conditional probability for displacement, and this displacement information may be acquired using NMR, a process which is discussed in chapters 4 and 5.

This analysis begins by introducing the probability density function $p(x, t)$, which gives the probability of finding a particle at position $x$ at time $t$. The conditional probability $P\left(x_{0}, t_{0} \mid x_{1}, t_{1}\right)$ is then defined as the probability of finding a particle at position $x_{1}$ at time $t_{1}$ given it was at position $x_{0}$ at time $t_{0}$. It is noted that it does not matter where the particle has been for $t<t_{0}$, and such a process which is indifferent to history is known as Markovian, an important property for this analysis.

To determine the probability $p\left(x_{1}, t_{1}\right)$ of finding a particle at some time $t_{1}$ and position $x_{1}$, the product of the probability density and the conditional probability is used, and 
integrated over all possible starting positions such that

$$
p\left(x_{1}, t\right)=\int p\left(x_{0}, t_{0}\right) P\left(x_{0}, t_{0} \mid x_{1}, t_{1}\right) d x_{0} .
$$

This equation states that the probability of finding a particle at some time $t=t_{1}-t_{0}$ will be the product of the probability of finding the particle at some starting point $x_{0}$ at time $t_{0}$ with the conditional probability of finding the particle at position $x_{1}$ some time $t$ later, given it was at position $x_{0}$ at time $t_{0}$, integrated over all possible starting points $x_{0}$. Since this process is Markovian, the position in time does not matter, only the displacement, and the conditional probability for such a situation is further expressed as $P\left(x \mid x^{\prime}, t\right)$ for an initial position $x$, final position $x^{\prime}$, with displacement in time $t$.

Einstein showed that the probability density $p\left(x^{\prime}, t\right)$ obeyed Fick's laws by considering small steps in space and time under Taylor expansions, the analysis of which is beyond the scope of this section. This analysis proceeds using Einstein's result claiming $p\left(x^{\prime}, t\right)$ satisfies Fick's laws for the initial condition $p(x, 0)$, and the relationship

$$
p\left(x^{\prime}, t\right)=\int p(x, 0) P\left(x \mid x^{\prime}, t\right) d x .
$$

By inserting the expression for $p\left(x^{\prime}, t\right)$ in Eq. 2.5 in place of $n(x, t)$ for Fick's second law in Eq. 2.3, and allowing $\nabla^{2}$ to operate on the $x^{\prime}$ variable as it is a Markovian process and may operate on either position variable, the relation

$$
\int p(x, 0) \frac{\partial P\left(x \mid x^{\prime}, t\right)}{\partial t} d x=D \int p(x, 0) \frac{\partial^{2} P\left(x \mid x^{\prime}, t\right)}{\partial x^{\prime 2}} d x
$$

is obtained. Taking the derivative of each side with respect to $x$, and dividing through by $p(x, 0)$, it can be shown that the conditional probability also obeys Fick's laws.

$$
\frac{\partial P\left(x \mid x^{\prime}, t\right)}{\partial t}=D \frac{\partial^{2} P\left(x \mid x^{\prime}, t\right)}{\partial x^{\prime 2}}
$$

This relationship will hold in other dimensions as well, and assuming the medium is isotropic such that diffusion is constant with respect to direction, this relationship may 
be rewritten as

$$
\frac{\partial}{\partial t} P\left(\mathbf{r} \mid \mathbf{r}^{\prime}, t\right)=D \nabla^{2} P\left(\mathbf{r} \mid \mathbf{r}^{\prime}, t\right)
$$

Again, noting the process in question is Markovian, the position variable on which the $\nabla^{2}$ operator is acting is not specified as it may act on either.

For free diffusion in which restriction is not experienced, the initial condition as knowledge of the exact location of a particle where $P\left(\mathbf{r} \mid \mathbf{r}^{\prime}, t\right)=\delta\left(\mathbf{r}-\mathbf{r}^{\prime}\right)$ for any $\mathbf{r}$ or $\mathbf{r}^{\prime}$ can be used. Applying this initial condition to Eq. 2.8, and solving for the conditional probability,

$$
P\left(\mathbf{r} \mid \mathbf{r}^{\prime}, t\right)=(4 \pi D t)^{-3 / 2} \exp \left\{-\frac{\left(\mathbf{r}-\mathbf{r}^{\prime}\right)^{2}}{4 D t}\right\}
$$

This solution for the conditional probability is the propagator for free diffusion, showing a Gaussian distribution which spreads over time, and the likelihood of finding the given particle in space and time.

\subsubsection{The Average Propagator}

When an ensemble of particles is considered and all displacements in space $\mathbf{R}$ are accounted for, the probability distribution for displacements is represented by the average propagator $\bar{P}(\mathbf{R}, t)$. This average propagator is represented as

$$
\bar{P}(\mathbf{R}, t)=\int p(\mathbf{r}) P(\mathbf{r} \mid \mathbf{r}+\mathbf{R}, t) d \mathbf{r},
$$

where $\mathbf{r}$ represents the position of the particle at time $t=0$, and $\mathbf{r}+\mathbf{R}$ represents the position of the particle at time $t$. Again, for free diffusion, solving for Eq. 2.8, the solution is expressed as

$$
\bar{P}(\mathbf{R}, \Delta)=(4 \pi D \Delta)^{-3 / 2} \exp \left\{-\frac{\mathbf{R}^{2}}{4 D \Delta}\right\}
$$

where $\Delta$ replaces $t$ as the observation time over which displacements are considered. The average propagator for free diffusion along the $z$-axis is plotted on the left of Fig. 2.1 for increasing time. As time increases, the average propagator spreads out as larger displacements become more probable. If uniform flow along the $z$ direction is included, 

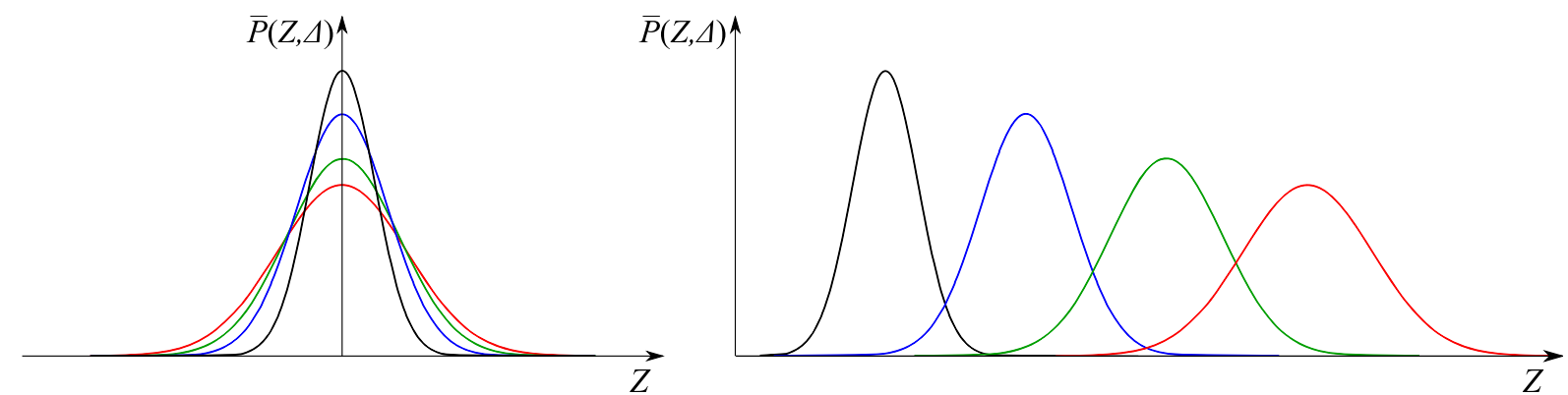

Figure 2.1: Left: Average propagator for free diffusion. As time evolves, the average propagator spreads as larger displacements become increasingly probable. Right: Average propagator for uniform flow along the $z$ direction and diffusion. As time evolves, the propagator spreads due to diffusion and is shifted further along the direction of flow.

this will result in a shift of the diffusion propagator along the direction of flow as time evolves, and is shown on the right of Fig. 2.1. For flow fields which are not uniform, and diffusion between flow lines (dispersion) cannot be neglected, further considerations or simulations are required to predict the average propagator [65]. However, if dispersion can be neglected, the average propagator will be the convolution of the diffusion propagator with the flow propagator.

\subsubsection{The Average Propagator for Pipe Flow}

The propagator measurement technique presented in this thesis was tested by measuring the average propagator of laminar flow of water through a cylindrical pipe, and therefore the theoretical average propagator for such a flow must be determined to verify the experimental results presented later in this thesis.

The flow field inside a pipe for laminar flow of a Newtonian fluid is a parabolic velocity profile described by $v(r)=v_{0}\left(1-r^{2} / a^{2}\right)$, where $a$ is the radius of the pipe, $v_{0}$ is the maximum velocity at the centre of the pipe, $r$ the distance from the axis of the pipe, and the flow direction is taken along the $z$-axis. The average propagator for pipe flow can be calculated by evaluating the equation

$$
\bar{P}_{\text {flow }}(\mathbf{R}, \Delta)=\int_{0}^{a} \int_{0}^{2 \pi} \int_{-L / 2}^{L / 2} r P(r, \theta, z) \times P(r, \theta, z \mid r+R, \theta+\Theta, z+Z, \Delta) d z d \theta d r,
$$


where $L$ is the length of the pipe, $L \gg a$, and $P(r, \theta, z)$ is the probability of finding a particle at the given position [66]. By evaluating this Eq. 2.12, it can be shown that the average propagator for this flow is the well known hat function with equal probability of displacements for $0 \leq Z \leq v_{0} \Delta$.

For the average propagator measurements presented in this research, a thin slice volume of flow is selected for the measurement, with the slice profile modulated by a Gaussian. Assuming a slice is selected along the $x$-axis, the probability a particle will be included in the measurement is

$$
P(x) \propto \exp \left\{\frac{-x^{2}}{b}\right\}
$$

where $b$ is related to the full width at half maximum $(F W H M)$ of the slice by

$$
b=\frac{F W H M}{\sqrt{2 \ln (2)}} .
$$

Converting $P(x)$ into polar coordinates and applying the flow profile for pipe flow, Eq. 2.12 can be rewritten as

$$
\bar{P}_{\text {flow }}(\mathbf{R}, \Delta) \propto \int_{0}^{a} \int_{0}^{2 \pi} \int_{-L / 2}^{L / 2} r \exp \left\{\frac{-r^{2} \cos ^{2}(\theta)}{b}\right\} \delta(R) \delta(\Theta) \delta\left[Z-v_{0}\left(1-\frac{r^{2}}{a^{2}}\right) \Delta\right] d z d \theta d r .
$$

Evaluating the integrals in $\theta$ and $z$, and dropping all constants which only relate to normalisation for a probability distribution, Eq. 2.15 can be rewritten as

$$
\bar{P}_{\text {flow }}(Z, \Delta) \propto \int_{0}^{a} r \exp \left\{\frac{-r^{2}}{2 b}\right\} I_{0}\left(\frac{r^{2}}{2 b}\right) \delta\left[Z-v_{0}\left(1-\frac{r^{2}}{a^{2}}\right) \Delta\right] d r
$$

where $I_{0}$ is the modified Bessel function of the first kind. A change of variables can then be made such that $\alpha=v_{0}\left(1-r^{2} / a^{2}\right) \Delta$, any constants contributing to the amplitude ignored, and Eq. 2.16 rewritten again as

$$
\bar{P}_{\text {flow }}(Z, \Delta) \propto \int_{0}^{v_{0} \Delta} \exp \left\{\frac{-a^{2}}{2 b}\left(1-\frac{\alpha}{v_{0} \Delta}\right)\right\} I_{0}\left[\frac{a^{2}}{2 b}\left(1-\frac{\alpha}{v_{0} \Delta}\right)\right] \delta(Z-\alpha) d \alpha .
$$


Evaluating Eq. 2.17, the average propagator for a Gaussian slice selection of a parabolic flow profile in a circular pipe is obtained (Eq. 2.18).

$$
\bar{P}_{\text {flow }}(Z, \Delta) \propto \exp \left\{\frac{-a^{2}}{2 b}\left(1-\frac{Z}{v_{0} \Delta}\right)\right\} I_{0}\left[\frac{a^{2}}{2 b}\left(1-\frac{Z}{v_{0} \Delta}\right)\right]
$$

To obtain the full average propagator, the effects of diffusion must be included. For an observation time $\Delta$ of $35 \mathrm{~ms}$, the diffusion length $d_{l}=\sqrt{2 D \Delta} \approx 12 \mu \mathrm{m}$. For a pipe radius $a=9 \mathrm{~mm}, d_{l} \ll a$, and the diffusion length will result in minimal dispersion and sampling of different flow lines for a given particle. Any effects of diffusion along the radial direction can therefore be ignored. As derived in Eq. 2.11, but now only considering displacement along the $z$ direction, the average propagator for free diffusion is the well know Gaussian propagator with the form

$$
\bar{P}_{D}(Z, \Delta) \propto \exp \left\{\frac{-Z^{2}}{4 D \Delta}\right\}
$$

To account for diffusion in the total average propagator in this low mixing regime for a Gaussian slice selection of pipe flow, the probability of a particle undergoing displacement $Z$ during the observation time will be the probability of displacement $Z^{\prime}$ due to flow, times the probability of displacement $Z-Z^{\prime}$ due to diffusion, integrated over all possible flow displacements $Z^{\prime}$. This is simply the convolution of the two propagators

$$
\bar{P}(Z, \Delta)=\bar{P}_{\text {flow }} \otimes \bar{P}_{D}
$$

and can be expressed in integral form as

$$
\bar{P}(Z, \Delta)=\int_{0}^{v_{0} \Delta} \bar{P}_{D}\left(Z-Z^{\prime}, \Delta\right) \bar{P}_{\text {flow }}\left(Z^{\prime}, \Delta\right) d Z^{\prime}
$$

Using the result from Eq. 2.18, and the propagator for free diffusion from Eq. 2.19, this expression can be evaluated numerically to obtain the average propagator for Gaussian slice selection of pipe flow where dispersion can be neglected. In Fig. 2.2 the average propagators for the given conditions are calculated and plotted for increasing flow rates. 


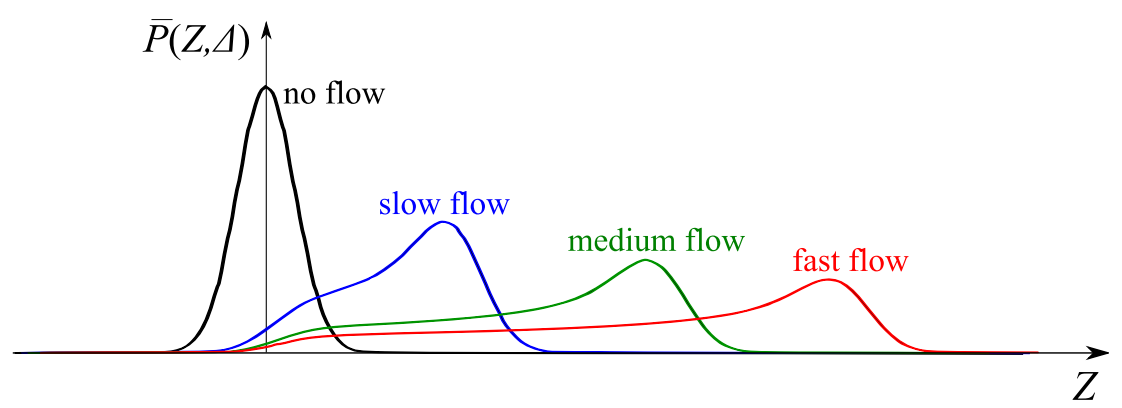

Figure 2.2: Average propagators for a Gaussian slice selection of laminar water flow through a circular pipe for increasing flow rates and constant observation time $\Delta$. For no flow, an average free diffusion propagator is observed which is symmetric in shape. As flow is introduced, the average propagator becomes the convolution of the average free diffusion propagator and the average flow propagator. With increasing flow, the shape of the average propagator becomes more skewed as the slice selection has excluded some of the low velocity regions of flow near the walls of the pipe, but included the higher velocity regions near the pipe axis, and the large displacements due to flow begin to dominate the smaller displacements due to diffusion.

As the flow rate is increased, larger displacements become more probable. The black curve corresponds to no flow, and this average propagator represents displacements due to diffusion alone. It is also noted that even for the highest flow rate shown in red, displacements in the negative $z$ direction are still possible. These displacements are due to particles residing near or at the walls. Since there is no flow velocity at the wall, diffusion of these particles can result in a displacement which opposes the direction of net flow.

\subsubsection{The Propagator for Restricted Diffusion}

Restricted diffusion is experienced when the diffusive motion of particles is constrained or limited by boundaries such as the pore space boundaries in a porous medium. For observation times where the particles experience this restriction, the mean squared displacement will be less than the expected displacement for free diffusion, and for closed geometries in which the particles are diffusing, the mean squared displacement will plateau, representing a length scale determined by the pore size distribution. The probability of finding a 
particle having undergone a displacement in time for such a situation can be expressed by the propagator.

The propagator for diffusion within porous media will be affected by the pore space in which it resides. Solutions for the propagator can be complex, but have been solved for ideal geometries such as cylindrical, spherical, and planar boundaries [67]. The simplest case involves diffusion which is restricted by a flat, smooth, reflecting boundary, resulting in a propagator which is the sum of a Gaussian and the portion of the Gaussian reflected by the pore boundary. In general, the Green's function representing the propagator must satisfy the diffusion equation

$$
\frac{\partial P\left(\mathbf{r} \mid \mathbf{r}^{\prime}, t\right)}{\partial t}=D_{0} \nabla^{2} P\left(\mathbf{r} \mid \mathbf{r}^{\prime}, t\right),
$$

with boundary conditions

$$
D_{0} \hat{n} \cdot \nabla P\left(\mathbf{r} \mid \mathbf{r}^{\prime}, t\right)+\left.\rho P\left(\mathbf{r} \mid \mathbf{r}^{\prime}, t\right)\right|_{\mathbf{r} \in S}=0
$$

subject to the initial conditions

$$
P\left(\mathbf{r} \mid \mathbf{r}^{\prime}, 0\right)=\delta\left(\mathbf{r}-\mathbf{r}^{\prime}\right)
$$

where $\rho$ is the surface relaxivity of the pore. One method of solving this is through a superposition of eigenfunctions using an eigenfunction expansion

$$
P\left(\mathbf{r} \mid \mathbf{r}^{\prime}, t\right)=\sum_{n=1}^{\infty} \psi_{n}(\mathbf{r}) \psi_{n}\left(\mathbf{r}^{\prime}\right) e^{-t / T_{n}}
$$

given the eigenfunctions $\psi_{n}$ also satisfy

$$
D \nabla^{2} \psi_{n}=\frac{-\psi_{n}}{T_{n}}
$$

and the boundary conditions

$$
D \hat{\mathbf{n}} \cdot \nabla \psi_{n}+\left.\rho \psi_{n}\right|_{\mathbf{r} \in S}=0,
$$


where $\hat{\mathbf{n}}$ is the unit vector normal to the surface of the pore and $\mathbf{r} \in S$ denotes that $\mathbf{r}$ belongs to the surface $S$ of the pore [27].

For diffusion at short times where the diffusion length is much less than the mean pore size, a perturbation expansion of the propagator may be used to obtain an expansion for the diffusion coefficient which is also valid at short times. This expansion uses a Laplace transform of the propagator and boundary conditions to derive a perturbation series. The time dependent apparent diffusion coefficient $D_{a p p}(t)$, which is related to the mean squared displacement $\sigma^{2}$ of particles through

$$
\sigma^{2}=2 n t D_{a p p}(t)
$$

can be shown to obey the relationship[13]

$$
\frac{D_{a p p}(t)}{D_{0}}=1-\frac{4}{3 d \sqrt{\pi}} \frac{S}{V_{p}}\left(D_{0} t\right)^{1 / 2}+\frac{\rho S t}{6 V_{p}}+O\left[\left(D_{0} t\right)^{3 / 2}\right],
$$

where $d$ is the number of dimensions in which diffusion is restricted, and $n$ is the number of dimensions in which displacement is measured. This result allows for partially absorbing boundary conditions where $\rho$ is the surface relaxivity of the pore-surface interface.

\subsection{Porous Media}

\subsubsection{Definition of Porous Media}

Porous media can be defined as solid matrices containing spaces in which liquid or gas may be contained. The pore space containing the liquid or gas is often continuous, like the matrix, but may contain closed pores in which the liquid or gas has been trapped upon formation of the matrix.

The matrix can be of homogeneous or heterogeneous nature as shown in Fig. 2.3. This often depends on the size of the volume in question, since some matrices will appear homogeneous on a large scale, but heterogeneous when a small volume is analysed, and vice versa. In addition to the level of homogeneity, porous media can be defined by a number 


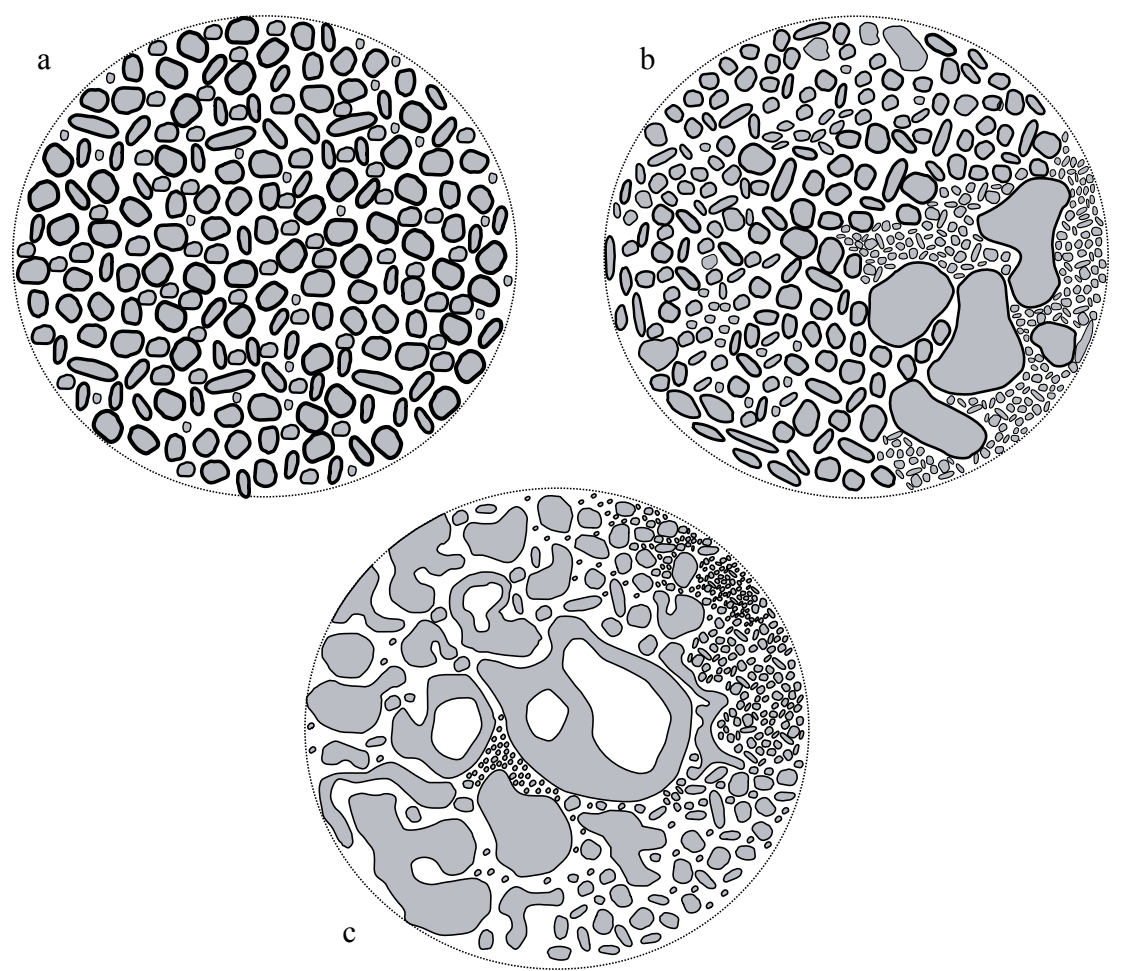

Figure 2.3: Cross sections of a generic porous media. a) Homogeneous porous media. b) Heterogeneous porous media. c) Highly heterogeneous porous media containing some closed pores.

of characteristics relating to the internal structure. In the following, different ways in which porous media are characterised and the associated properties of such matrices are presented.

\subsubsection{Properties of Porous Media}

There are a number of properties which help classify and describe porous media, namely the porosity, homogeneity, pore size distribution, pore geometry, tortuosity, and surface to volume ratio of the pore space. The level of homogeneity is associated with the porosity and pore size distribution throughout the matrix. Although the porosity $\phi$, the fraction of pore space to the total volume, may not change as random volumes are analysed, the pore size and shape may. The term 'pore' can be difficult to define as the pore space 
is usually interconnected and may represent a complex network of irregular pore shapes with connective throats varying in shape and size. This is very different from an ideal matrix whose pores are spherical with regular size and connected by well defined throats. It may be stated that for a constant sized volume chosen randomly from the matrix, if the pore size distribution and porosity are constant across a particular region in the matrix, that the matrix, across that region, is homogeneous.
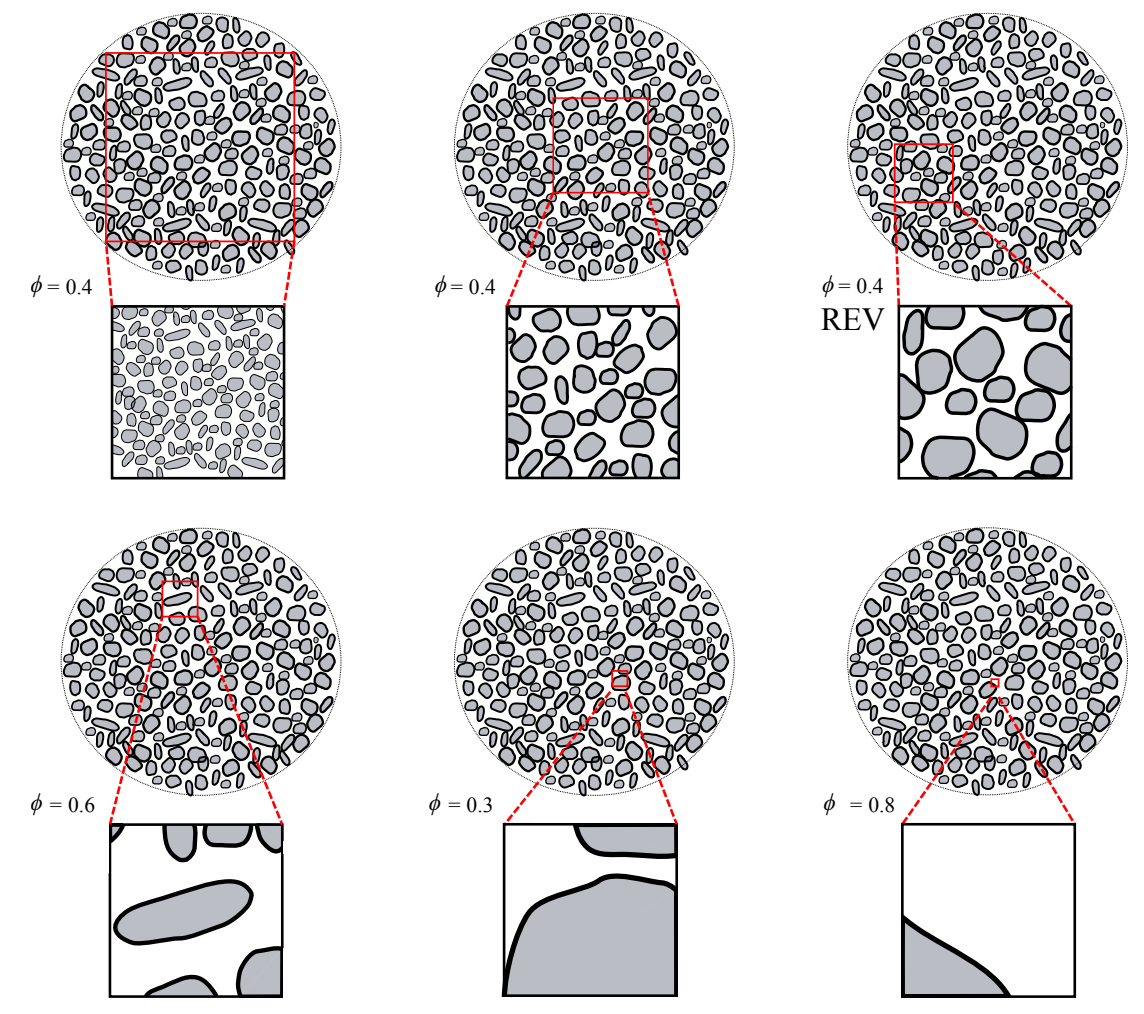

Figure 2.4: Random volumes of decreasing size are taken from the homogeneous matrix shown in Fig. 2.3. As the volume size is decreased, the porosity of the volume begins to fluctuate. The smallest volume taken before porosity fluctuation begins is the REV.

When the size of a random volume element is varied within a matrix, the porous medium may appear heterogeneous or homogeneous. If a matrix is composed of regions of differing pore size distribution and porosity, the matrix may appear heterogeneous for volumes smaller or comparable to the size of these regions, but homogeneous for volumes much larger than the size of these regions. The most common way of classifying such effects is 
through a representative elementary volume (REV). The REV gives a volume size which is the lowest volume such that the matrix appears homogeneous. By taking smaller and smaller unit volumes of the matrix and determining the porosity and pore size distribution of each volume, the volume at which the matrix starts to lose homogeneity can be defined as the REV (Fig. 2.4).

For porous media containing closed pores, the effective porosity of the matrix can also be defined. The effective porosity is defined as the drainable porosity, or the porosity through which flow may occur. Since some of the water or gas can be bound in closed pores, this volume is omitted from the porosity calculation to give the effective porosity. It is further noted that the porosity through which flow may occur is not necessarily equal to the drainable porosity. Some of the pore space may branch off from the main network and result in a dead end. Although this pore space is not closed, it would contribute virtually nothing to the flow characteristics through the matrix.

Another characteristic of porous media is that the porosity may remain constant for varying pore size. Considering a random bead pack of uniform diameter spherical beads, the porosity of the matrix will be constant with respect to the diameter of the beads $\phi=0.36-0.375$. Although the porosity of bead packs remains constant for different bead diameters, the pore and throat size will vary. Therefore, other characteristics must be defined to provide a more complete description of the matrix such as the pore size distribution, tortuosity, and surface to volume ratio of the pore space.

Pore size distributions may be used to give a description of how the pore spaces vary in size inside a matrix. Since the pore structure is interconnected, and may be so in a fashion that varies greatly in pore openings, throats, and pore geometry, where a pore begins and ends, or how to define its size, can vary widely depending on the measurement and definition. One common definition of the pore size is given as the diameter of the largest sphere which will fit inside of the pore [56].

The tortuosity of a matrix provides a description of how twisted the interconnectivity of the pore space in the matrix is. This twisting, or lack of direct pathways between two points in the matrix, will in part define how fluid or gas flows and diffuses through the 
pore space. The tortuosity $\alpha$ may be defined as

$$
\alpha=\left(L / L_{T}\right)^{2}
$$

where $L$ is the straight line distance between two points and $L_{T}$ is the length of the tortuous path [56], as illustrated in Fig. 2.5.
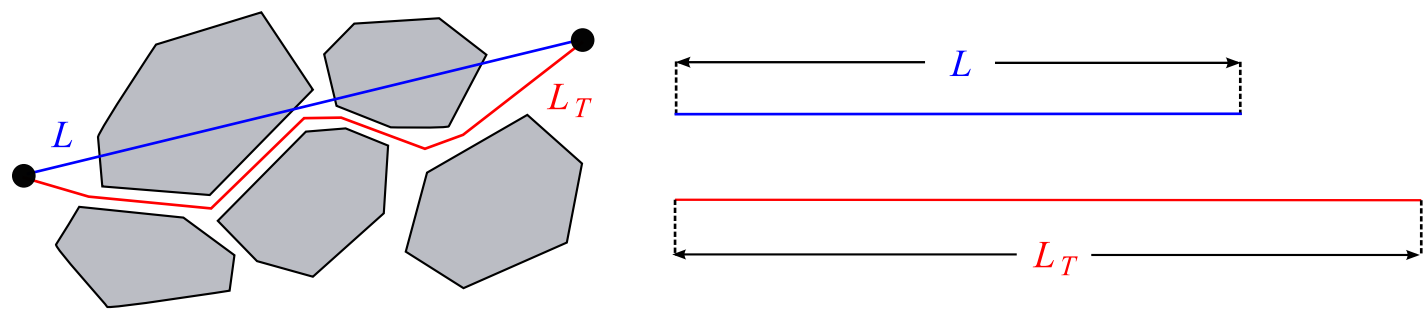

Figure 2.5: Left: Straight line segment between two points shown in blue, and the tortuous path shown in red. Right: Comparison of lengths between the straight line and tortuous paths.

Another definition of the tortuosity involves the formation factor $F$, which is the ratio of the conductivity of a bulk fluid to the conductivity of the same fluid inside a matrix whose pore space has been saturated. This relation for the tortuosity may be defined as

$$
\alpha=F \phi
$$

where $\phi$ is again the porosity of the matrix. The tortuosity of a porous medium may also be defined through the apparent diffusion coefficient of the fluid contained within the pore space. Defining the apparent diffusion coefficient as $D_{a p p}$, the tortuosity of a porous medium may be expressed as

$$
\alpha=D_{\text {app }}(\infty) / D_{0}
$$

where $D_{0}$ is the free diffusion coefficient.

The measurement of the time dependent apparent diffusion coefficient allows measurement of the tortuosity of a porous medium in its long time limit, and the surface to volume ratio in its short time limit. In a porous medium saturated with a fluid which 
is diffusing within the pore space, as time evolves, more and more nuclei will experience diffusive restriction by the walls defining the pore space.
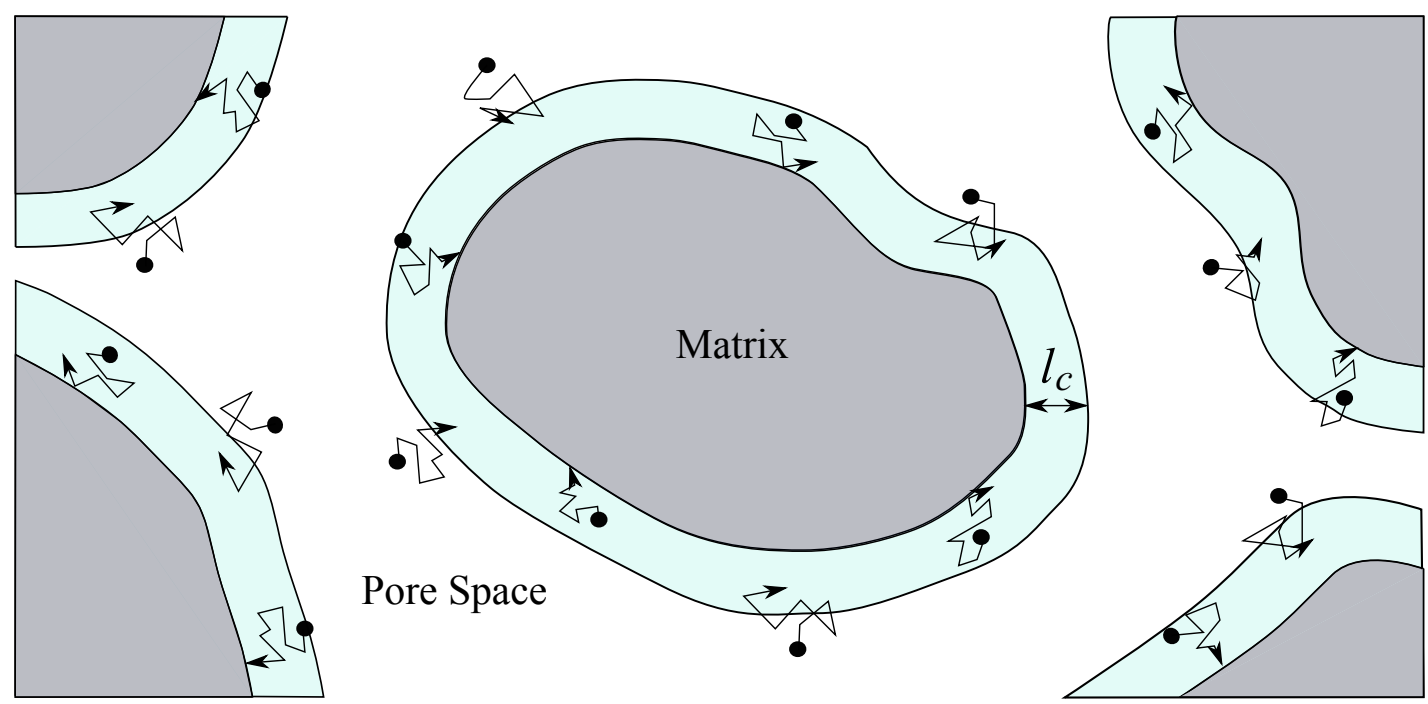

Figure 2.6: A matrix grain shown in gray, and the associated volume of pore space shown in light blue which will experience diffusive restriction due to the matrix grain. Only nuclei within the distance $l_{c}(t)$ will see the walls of the matrix grain.

Taking the characteristic diffusion length $d_{l}(t)=\sqrt{D_{0} t}$ at some time $t$, and assuming this length is small compared to the mean pore diameter $d_{p}$ such that $d_{l}(t) \ll d_{p}$, the volume of the nuclei which have experienced diffusive restriction due to locally flat pore boundaries of surface area $S$ is expressed as $S d_{l}(t)$, and the fraction of nuclei experiencing these boundaries as $S d_{l}(t) / V_{p}$ where $V_{p}$ is the pore space volume. As shown by by Mitra et al $[13,27]$, the time dependent apparent diffusion coefficient may be expressed as

$$
\frac{D_{a p p}(t)}{D_{0}}=1-\frac{4}{3 d \sqrt{\pi}} \frac{S}{V_{p}}\left(D_{0} t\right)^{1 / 2}+O\left(D_{0} t\right)
$$

where $d$ is the number of dimensions in which diffusive restriction is experienced. With this relationship, in the short time limit, the slope of $D_{a p p}(t) / D_{0}$ plotted against $t^{1 / 2}$ will yield the surface to volume ratio of the pore space. 


\subsection{Conclusions}

A basic description of self-diffusion, the diffusive process in the absence of concentration gradients, has been presented, along with implications on how the diffusive process is influenced when the diffusing particles reside in the pore space of a porous medium. A definition of porous media has also been provided with defining properties used to characterise the pore space of the porous medium.

The concept of the average propagator, the probability distribution for displacement of particles in space and time, has been introduced. Its use in the characterisation of flow and characterisation of porous media through measurement of restricted diffusion is a central part of this research. The average propagator is measurable through the experimental techniques presented in chapter 4 and chapter 5. Before discussing the experimental techniques for measurement of the average propagator and diffusion, the basics of nuclear magnetic resonance are presented next in chapter 3. 


\section{Chapter 3}

\section{Nuclear Magnetic Resonance}

\subsection{The NMR Signal}

\subsubsection{Introduction}

The basis of nuclear magnetic resonance was proposed in 1946 by Felix Bloch [68] in his paper "Nuclear Induction," and has been evolving into increasingly complicated and elaborate techniques allowing measurement of complex behaviour and interactions of nuclear species with themselves and their surroundings. NMR has found a home in multiple facets of science, industry, and medicine, proving to be a diverse and elegant measurement technique. In this chapter the basics of NMR and the inherent restrictions for experimental measurements are presented, with more detailed descriptions found elsewhere [69-71].

\subsubsection{Nuclear Spin}

The phenomena known as nuclear magnetic resonance stems from the quantum mechanical property of spin and its associated magnetic moment. Spin is a name given to the angular momentum possessed by particles and nuclei. This spin is not associated with a rotational movement as in classical physics, but is solely a quantum mechanical attribute of nuclei and certain particles.

Nuclei are composed of protons and neutrons whose spins are combined to determine the 
total spin of the nucleus. Both protons and neutron possess a spin of $1 / 2$. If a proton is bound with another proton, their spins will pair such that one has a spin of $+1 / 2$ and the other a spin of $-1 / 2$, giving the nucleus a total spin of 0 . The spins of protons will pair with spins of other protons, and those of neutrons with neutrons, making the total spin of the nucleus a sum of the spins of the unpaired protons and unpaired neutrons. For
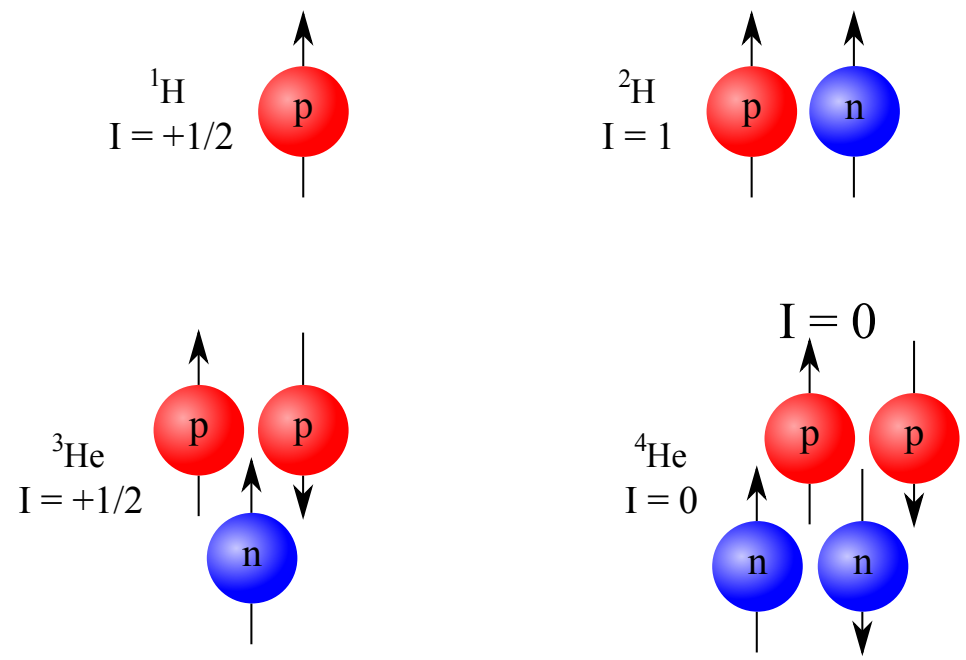

Figure 3.1: Nuclear Spin Composition

the cases shown in Fig. 3.1, determining the net spin of the nucleus is straight forward and simple. Since the model for nuclei is composed of shells similar to that of electronic structure, every second additional proton or neutron may not pair with the previous one to cancel out the net spin. Instead, additional protons or neutrons may further add to the total spin of the nucleus, resulting in some nuclei with a net spin $>1$.

With a non-zero net spin, and a magnetic moment associated with the spin, a nucleus with non-zero net spin will possess a non-zero magnetic moment. As seen by an outside observer, the net magnetic moment of a given nuclei will depend on the element in question as its strength will be affected by the shielding of its electronic structure and the nuclear composition. The most commonly used nucleus for NMR measurement is the hydrogen nucleus, composed of a single proton. Since protons have spin of $1 / 2$, this gives the hydrogen nucleus a total spin of $1 / 2$. 


\subsubsection{Polarisation}

Since a nuclear magnetic moment is associated with the spin state of each nucleus, spin polarisation is required to produce a net magnetic moment in the ensemble. Conventionally, polarisation is achieved through the technique of thermal polarisation in a static magnetic field. If one considers the magnetic moments in a nuclear ensemble, taking a classical perspective, in the absence of an external magnetic field, each magnetic moment will be oriented randomly in space. This random orientation of magnetic moments will result in a net magnetic moment which is equal to zero. Upon exposure to an external magnetic field, these magnetic moments, on average, partially align with the field. As alignment becomes energetically favourable, and the average direction of the magnetic moments becomes defined, a net magnetic moment will be produced. Assuming a semiclassical perspective on this process and considering the potential spin states, the nuclear spin will possess either an orientation of spin up or spin down for a spin one half $I=1 / 2$ nucleus, and a magnetic quantum number of $m= \pm 1 / 2$. In the absence of an external magnetic field, the population of these two states will be equal since they are energetically degenerate, and no net spin or magnetic moment will exist. When the ensemble is placed in an external magnetic field, the degeneracy of these spin states will be lifted. With the spin state aligned with the external magnetic field energetically favourable, it will possess a larger spin population than the anti-aligned state, and spin polarisation, and therefore a non-zero net magnetic moment, will exist.

The magnetic moment $\mu$ of a nucleus may be represented as a magnetic dipole. Since the nuclear magnetic dipole is directly related to the spin state of the nucleus, it has only two available directions with respect to the external magnetic field, either aligned or anti-aligned $m= \pm 1 / 2$ (Fig. 3.2). This yields energies of

$$
U= \pm \mu_{z} B
$$

making the aligned state slightly energetically favourable, creating an energy difference between the two states of

$$
\Delta U=2 \mu_{z} B .
$$



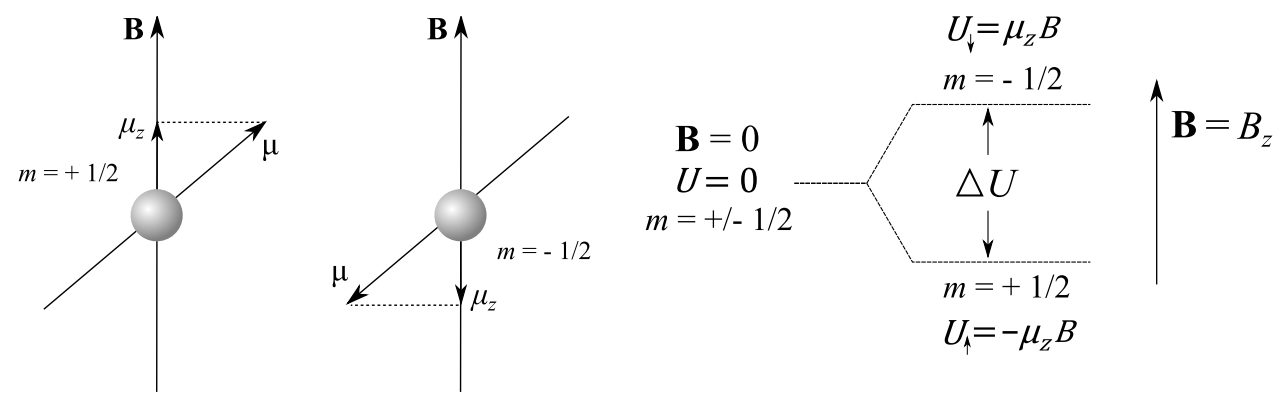

Figure 3.2: Left: Aligned and anti-aligned spin states for a spin 1/2 nucleus. Right: Energy levels associated with the two spin states. When an external magnetic field is applied, the degeneracy of the states is lifted, and an energy gap is created between the two states.

At thermal equilibrium, the polarisation $P$ of the nuclear ensemble can be calculated using the Boltzmann distribution

$$
P=\frac{N_{\uparrow}-N_{\downarrow}}{N_{\uparrow}+N_{\downarrow}}=\frac{\mathrm{e}^{-U_{\uparrow} / k_{B} T}-\mathrm{e}^{-U_{\downarrow} / k_{B} T}}{\mathrm{e}^{-U_{\uparrow} / k_{B} T}+\mathrm{e}^{-U_{\downarrow} / k_{B} T}} \approx \frac{\mu_{z} B_{0}}{2 k_{B} T},
$$

where $N_{\uparrow}$ is the population of the aligned state, $U_{\uparrow}$ is the energy of the aligned state, $N_{\downarrow}$ is the population of the anti-aligned state, $U_{\downarrow}$ is the energy of the anti-aligned state, $k_{B}$ is the Boltzmann constant, $T$ is the temperature, and $B_{0}$ is the strength of the static magnetic field produced by the NMR magnet.

\subsubsection{Excitation}

Once spin polarisation has been achieved to produce a non-zero magnetic moment in the nuclear population, radio frequency electromagnetic radiation is used to tip this net magnetic moment away from the external magnetic field $\mathbf{B}_{0}$. This process is known as spin excitation. A radio frequency $(\mathrm{RF})$ coil produces a field known as the $\mathbf{B}_{1}$ field, and is built to resonate at the Larmor frequency.

The Larmor frequency corresponds to the frequency at which the magnetic moment of a nucleus will precess in an applied magnetic field. This is a characteristic frequency determined by the strength of the NMR magnet $B_{0}$, any other additional magnetic fields, 
and the nucleus in question. Equating the energy gap between spin states of a nucleus in an applied magnetic field $B_{0}$ to the energy of electromagnetic radiation from the $\mathrm{RF}$ coil at the Larmor frequency, and neglecting the shielding effects of the atom's electrons,

$$
2 \mu_{z} B_{0}=\hbar \omega_{L}
$$

where $\omega_{L}$ is the Larmor frequency. The nuclear magnetic moment $\mu_{z}$ is also related to the nuclear spin by the gyromagnetic ratio $\gamma$ through

$$
\mu_{z}=\gamma L_{z}=\gamma \hbar / 2
$$

Substituting Eq. 3.5 into Eq. 3.4, the Larmor frequency is related to the gyromagnetic ratio by the external magnetic field

$$
\omega_{L}=\gamma B_{0}
$$

It is noted that this is the Larmor frequency for a nucleus experiencing only the $\mathbf{B}_{0}$ field, and it is the total magnetic field which will determine the Larmor frequency. The Larmor frequency for the nuclei experiencing only the $\mathbf{B}_{0}$ field from the NMR magnet is defined as an 'on resonance' frequency $\omega_{0}$ by

$$
\omega_{0}=\gamma B_{0}
$$

This will be of importance later as other magnetic fields are superimposed on the $\mathbf{B}_{0}$ field, and the Larmor frequency $\omega_{L}$ will vary from $\gamma B_{0}$.

Since each nucleus in the population possesses angular momentum and a magnetic moment, one can consider a unit volume with net angular momentum $\mathbf{L}$ and magnetisation $\mathbf{M}$ which are determined by the nuclear ensemble (Fig. 3.3).

The torque $\mathbf{T}$ produced on this unit volume may be expressed as

$$
\mathbf{T}=\frac{d \mathbf{L}}{d t}
$$




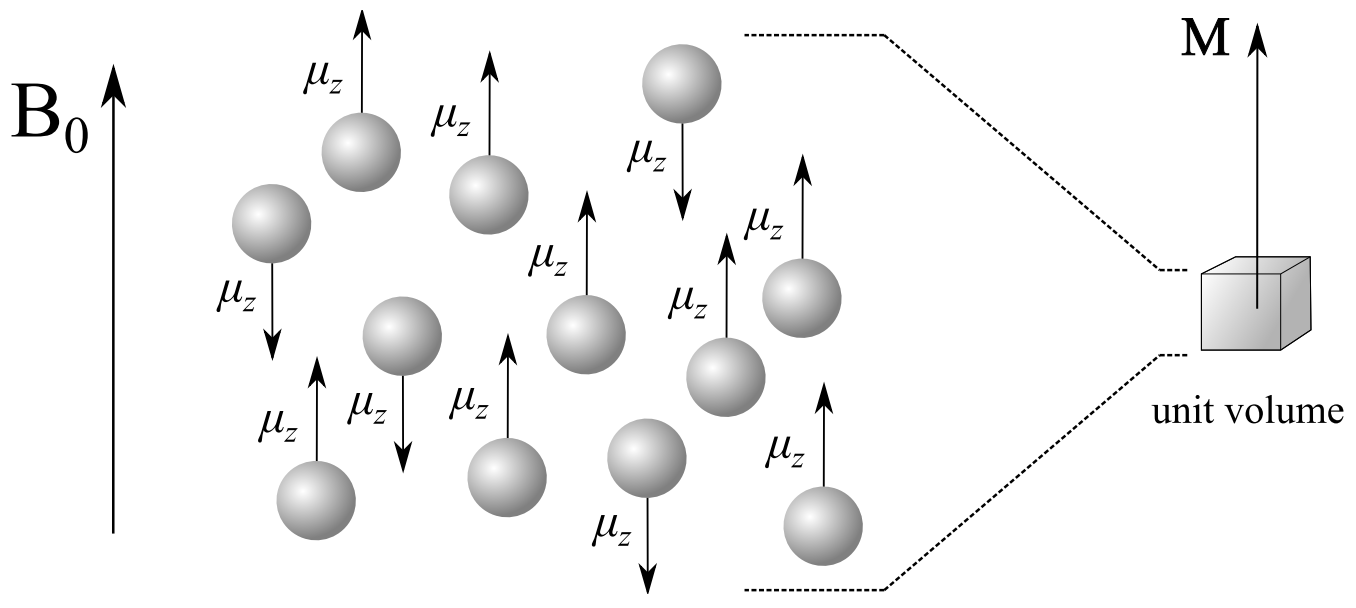

Figure 3.3: Unit volume with net magnetisation $\mathbf{M}$ parallel to its net angular momentum L.

where $\mathbf{L}$ is the spin angular momentum of the unit volume in question. Neglecting shielding of the external field $\mathbf{B}_{0}$ experienced at the nucleus due to its electrons, the torque is also related to magnetisation $\mathbf{M}$ through the equation

$$
\mathbf{T}=\mathbf{M} \times \mathbf{B}_{0}
$$

The magnetic moment of a nucleus is related to its spin angular momentum by the gyromagnetic ratio $\gamma$ where

$$
L_{z}=\frac{\mu_{z}}{\gamma}
$$

with $L_{z}$ representing the nuclear spin angular momentum and $\mu_{z}$ the nuclear magnetic moment. Similarly, the magnetic moment and spin angular momentum per unit volume may also be related to $\gamma$ by

$$
\mathbf{L}=\frac{\mathbf{M}}{\gamma}
$$

Substituting Eq. 3.11 and Eq. 3.8 into Eq. 3.9 yields a relationship of

$$
\frac{d \mathbf{M}}{d t}=\gamma\left(\mathbf{M} \times \mathbf{B}_{0}\right)
$$


To visualise how the magnetisation is tipped by an incident radio frequency electromagnetic field perpendicular to $\mathbf{B}_{0}$, a transformation into the rotating frame at the Larmor frequency can be made. In this rotating frame, precession of nuclear magnetic moments about $\mathbf{B}_{0}$ will disappear as the reference frame is rotating at the same rate. Since there appears to be no torque caused by $\mathbf{B}_{0}$ on a magnetic moment tipped away from $\mathbf{B}_{0}$, it is deemed a fictitious field in this frame and may be ignored in the rotating frame analysis. Any applied $\mathbf{B}_{1}$ field will have a polarisation which is linear, circular, or a combination of the two, with circular being more efficient but linear being more common. A linearly polarised $\mathbf{B}_{1}$ field may be expressed as two counter-rotating circularly polarised fields superimposed on one another.

$$
B_{1} \cos \left\{\omega_{0} t\right\}=\frac{B_{1}}{2} \exp \left\{\mathrm{i} \omega_{0} t\right\}+\frac{B_{1}}{2} \exp \left\{-\mathrm{i} \omega_{0} t\right\}
$$

In the rotating reference frame, only one of these terms will appear static, with the other appearing to rotate at twice the Larmor frequency. For this reason, the off resonance term appearing to rotate at $2 \omega_{0}$ in the rotating reference frame may be ignored. As a result, the following analysis for a circularly polarised $\mathbf{B}_{1}$ field will hold for a linearly polarised $\mathbf{B}_{1}$ field, with the only difference being a time varying magnitude of $\mathbf{B}_{1}$. In a reference frame rotating at the same frequency as the $\mathbf{B}_{1}$ field, the direction of $\mathbf{B}_{1}$ will appear static and analysis is simplified. The direction of the $\mathbf{B}_{1}$ field in the rotating frame is determined by the phase of the transmitter driving the RF coil, a parameter which is controlled experimentally. Upon application of the $B_{1}$ field at the Larmor frequency, in this case a circularly polarised field perpendicular to the $\mathbf{B}_{0}$ field, a torque will be experienced by the magnetic moment. The magnetisation will then rotate about $\mathbf{B}_{1}$ at an angular frequency of

$$
\omega_{1}=\gamma B_{1}
$$

and the magnetisation will undergo a tip angle $\theta$ of

$$
\theta=\omega_{1} t_{p}
$$


where $t_{p}$ is the duration of the RF pulse (Fig. 3.4).
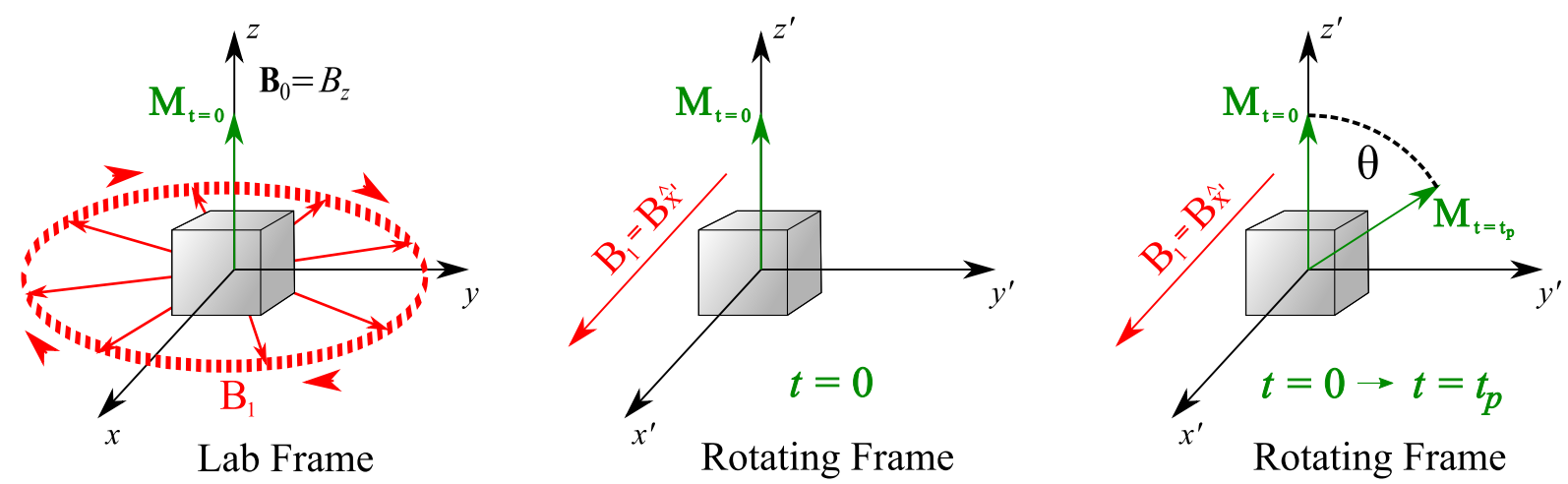

Figure 3.4: Application of a circularly polarised $\mathbf{B}_{1}$ field. Left: Lab frame at the beginning of the RF pulse. Centre: Reference frame rotating at $\omega_{0}$ at the beginning of the RF pulse. Right: During the RF pulse, the magnetisation experiences a torque about $\mathbf{B}_{1}$ and is rotated by an angle $\theta$ about the direction of the $\mathbf{B}_{1}$ field.

The magnetic field can be written as a sum of the $\mathbf{B}_{1}$ field along the $x^{\prime}$-axis and the $\mathbf{B}_{0}$ field along the $z^{\prime}$-axis, and Eq. 3.12 results in the relationships

$$
\begin{gathered}
\frac{d M_{x^{\prime}}}{d t}=\gamma M_{y^{\prime}}\left(B_{0}-\omega / \gamma\right) \\
\frac{d M_{y^{\prime}}}{d t}=\gamma B_{1}-\gamma M_{x^{\prime}}\left(B_{0}-\omega / \gamma\right) \\
\frac{d M_{z}}{d t}=-\gamma M_{y^{\prime}} B_{1}
\end{gathered}
$$

where $\omega$ is the true precession frequency of the magnetic moment due to slight off resonance excitation or any other magnetic fields present. These equations are an incomplete representation of the Bloch equations, used to classically describe quantum phenomena, and the evolution of spin ensembles in NMR. This interpretation is acceptable since the time dependence of quantum mechanical expectation values will evolve and adhere with classical equations of motion. Since the spin and magnetic moment vectors are always parallel to one another, Fig. 3.5 is an appropriate interpretation of the magnetic moment per unit volume in a nuclear ensemble for the given conditions [68].

Once the magnetic moment has been tipped away from $\mathbf{B}_{0}$, it will experience a torque 

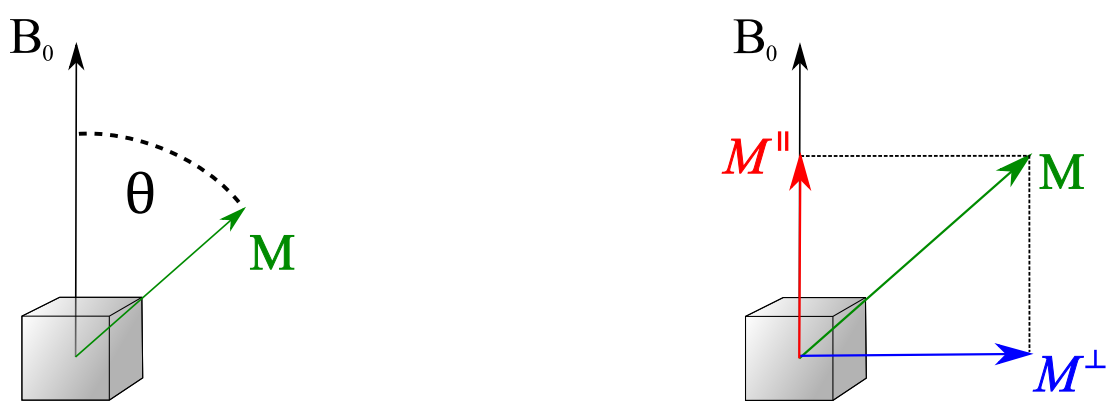

Figure 3.5: Left: The magnetisation has been tipped away from $\mathbf{B}_{0}$ by an RF pulse. Right: The magnetisation will have a component parallel to and perpendicular to the $\mathbf{B}_{0}$ field. The component $M^{\perp}$ will undergo precession about $\mathbf{B}_{0}$ due to the torque described by Eq. 3.12 .

about the external magnetic field. This torque will cause precession about $\mathbf{B}_{0}$ at the Larmor frequency. Of interest is the component of magnetisation which is perpendicular to the $\mathbf{B}_{0}$ field since it is the component which will undergo precession (Fig. 3.6).

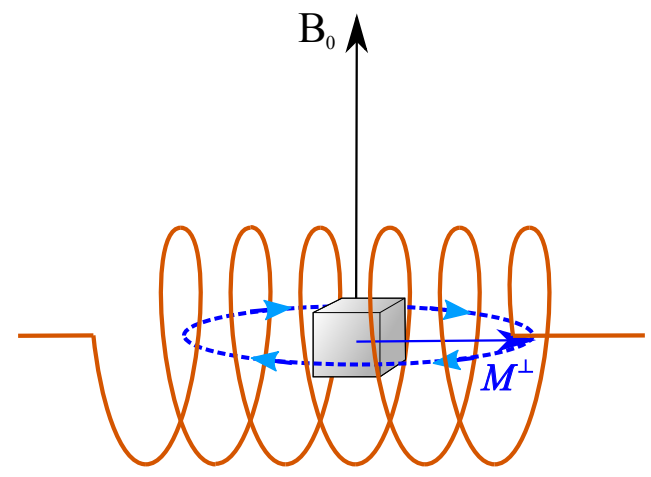

Figure 3.6: Example of a solenoid receiver coil used to measure the changing magnetic flux in space and time due to the perpendicular component of the excited magnetisation precessing about $\mathbf{B}_{0}$.

As the magnetisation precesses about $\mathbf{B}_{0}$, the perpendicular component of the magnetisation $M_{\perp}$ will cause the magnetic field to vary rapidly in space and time. This changing magnetic field is harnessed through electromagnetic induction to produce a signal voltage $E$ in an RF coil, which may or may not be the same coil used for excitation. The induced 
voltage in the receiver coil may be expressed as

$$
E=\frac{d \Phi_{B}}{d t} \propto M^{\perp}(t) \exp \left\{\mathrm{i} \omega_{0} t\right\}
$$

where $\Phi_{B}$ is the magnetic flux through the coil. An example of a simple solenoid receiver coil is shown in Fig. 3.6.

\subsection{Relaxation Effects}

\subsubsection{The FID and $T_{2^{*}}$ Relaxation}

After excitation of the nuclear ensemble where at least part of the net magnetisation is tipped perpendicular to $\mathbf{B}_{0}$, precession of this perpendicular component will induce a signal voltage in the receiver coil which decays in time. This signal is called the free induction decay (FID). The magnetisation giving rise to this signal can be expressed as

$$
M^{\perp}(t)=M^{\perp}(0) \exp \left\{-t / T_{2^{*}}\right\}
$$

where $T_{2^{*}}$ is a time constant characterising the rate of decay. Therefore, the induced voltage in the receiver coil can be expressed as

$$
E(t)=E_{0} \exp \left\{i \omega_{0} t\right\} \exp \left\{-t / T_{2^{*}}\right\},
$$

where $E_{0}$ is the signal amplitude directly after excitation. Conventionally the receiver is set on resonance $\omega_{0}$, and the amplitude of the oscillating voltage is measured (Fig. 3.7). The amplitude of the transverse component of the magnetisation, and therefore the signal voltage, decays in time due to what is known as $T_{2^{*}}$ relaxation. Since the precession frequency is directly proportional to the magnetic field strength, field inhomogeneities will cause the nuclei to precess at different frequencies. Depending on the location of the spins, some will precess slower, and some faster, resulting in the accumulation of phase relative to on resonance precession. Ideally, a magnet would produce a perfectly homogeneous magnetic field, but due to manufacture and design limitations, the field will always have 


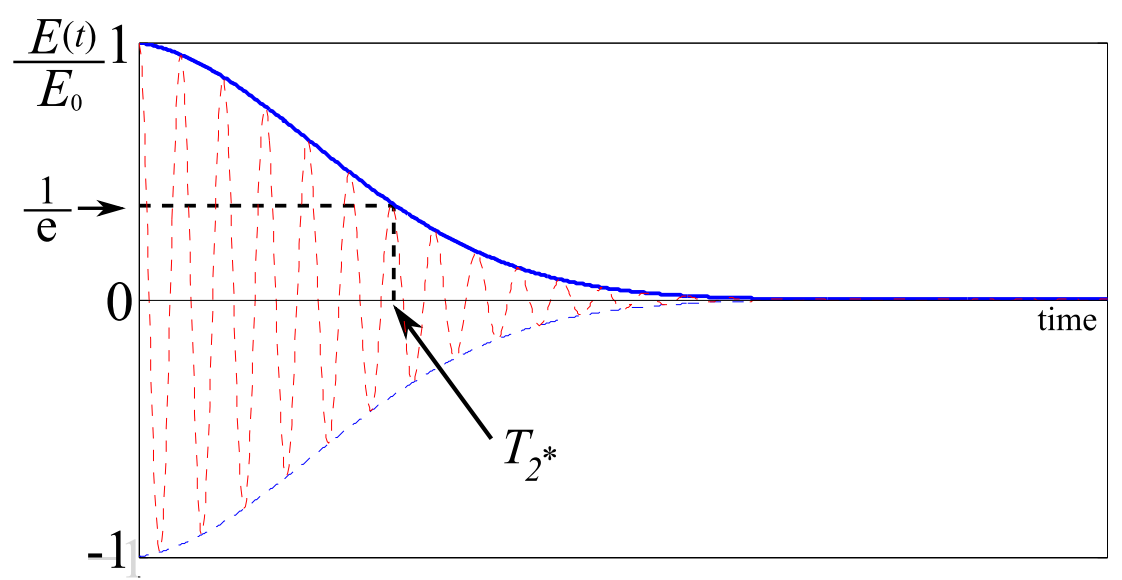

Figure 3.7: Example of a normalised free induction decay (FID) NMR signal. Signal frequency shown in dashed red is not to scale for illustrative purposes. With the receiver set on resonance, the dark blue curve represents the measured voltage.

slight variations. Mismatching of magnetic susceptibility between interfaces of fluid and any material or sample holder present can also contribute to field inhomogeneity.

The entire nuclear ensemble may be represented as a collection of unit volumes, and the magnetisation of a single unit volume tipped into the transverse plane may be expressed as the magnetisation density at that position evolved in time by a frequency dependent on the magnetic field at the given position $\mathbf{r}$, such that

$$
M^{\perp}(\mathbf{r}, t)=M_{0}^{\perp}(\mathbf{r}) \exp \{\mathrm{i} \omega(\mathbf{r}) t\}
$$

where $M_{0}^{\perp}(\mathbf{r})$ is the magnetisation density. In Fig. 3.8, multiple unit volumes are shown dephasing, each with their own spatially dependent frequency of precession $\omega(\mathbf{r})$. The spatially dependent precession frequency $\omega$ for a unit volume is given by

$$
\omega(\mathbf{r})=\omega_{0}+\Omega(\mathbf{r})
$$

with $\Omega(\mathbf{r})$ representing the offset frequency from resonance defined as

$$
\Omega(\mathbf{r})=\gamma\left(B(\mathbf{r})-B_{0}\right)
$$




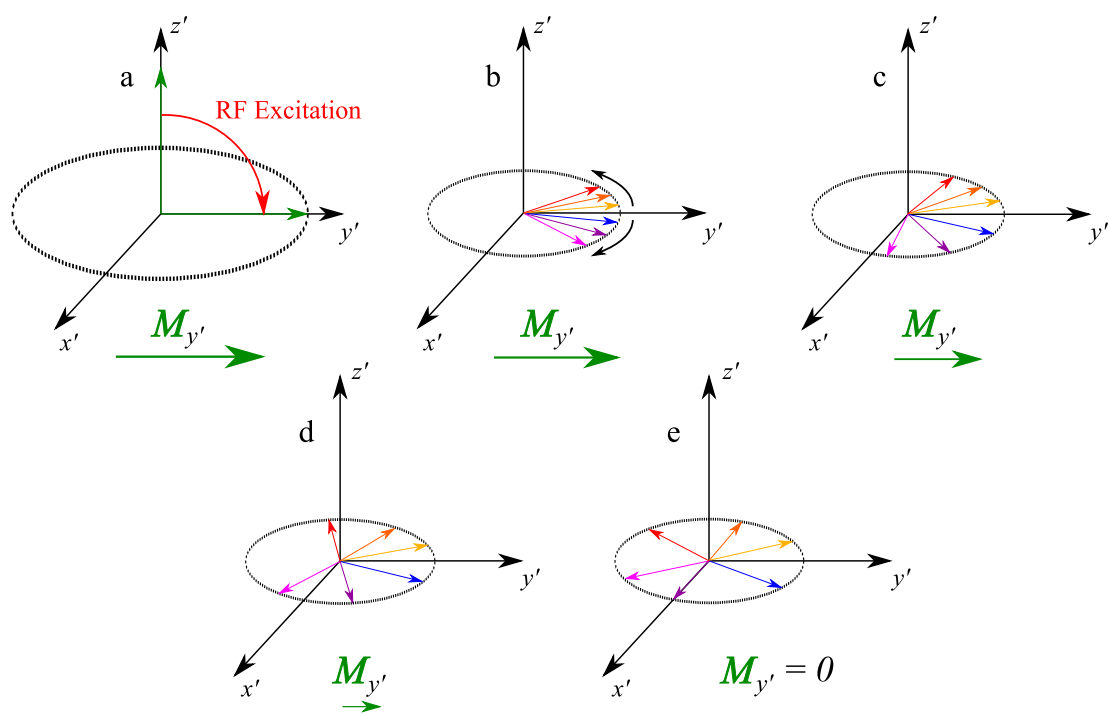

Figure 3.8: Time series phase evolution of the magnetisation of unit volumes at different positions in the NMR magnet. a) Excitation by a $90^{\circ} \mathrm{RF}$ pulse. b-d) Spins dephasing such that the constructive interference from magnetisation in each unit volume produces a decreasing net magnetisation. e) Spins are completely dephased and magnetisation from the unit volumes destructively interferes such that no net magnetisation exists in the transverse plane.

For convenience, Eq. 3.23 is substituted into Eq. 3.22 to arrive at

$$
M^{\perp}(\mathbf{r}, t)=M_{0}^{\perp}(\mathbf{r}) \exp \left\{\mathrm{i}\left(\omega_{0}+\Omega(\mathbf{r})\right) t\right\}
$$

The relation in Eq. 3.25 shows the accumulation of phase $\phi(\mathbf{r})=\Omega(\mathbf{r}) t$ with respect to the magnetisation precessing on resonance. The phase accumulation and the resulting $T_{2^{*}}$ relaxation may be accounted for by applying an $\mathrm{RF}$ pulse which rotates the magnetisation by $180^{\circ}$ about one of the transverse axes.

After an elapsed time of $t_{1}$, a given unit volume will have acquired a net phase shift with respect to resonance of

$$
\phi(\mathbf{r})=\Omega(\mathbf{r}) t_{1} .
$$




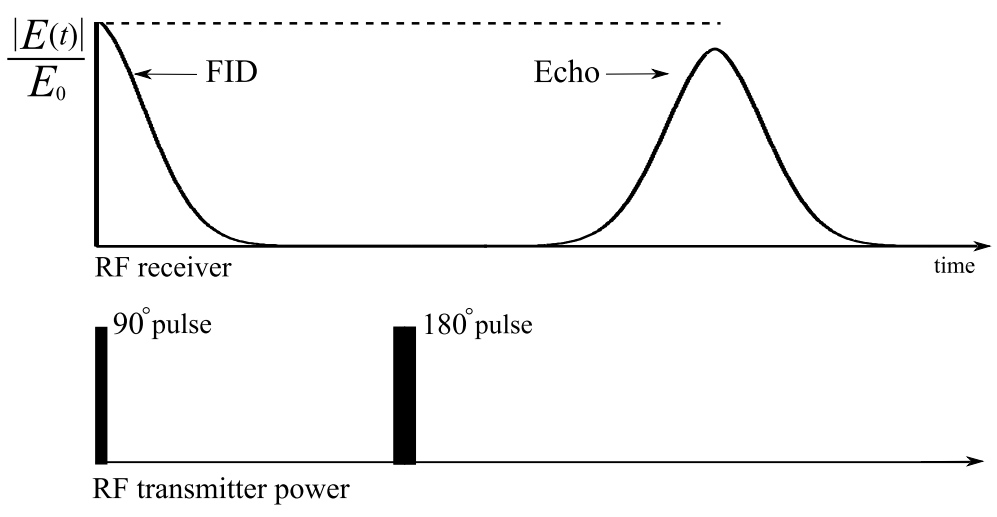

Figure 3.9: Top: Normalised RF receiver voltage showing FID and Hahn echo, with lettering corresponding to subfigures in Fig. 3.10. Bottom: RF transmitter power showing timing of $90^{\circ}$ and $180^{\circ}$ pulses.

Upon application of a $180^{\circ} \mathrm{RF}$ pulse of duration $\Delta t_{180^{\circ}}$, assuming $\Delta t_{180^{\circ}} \ll t_{1}$, this phase will switch in sign such that

$$
\phi(\mathbf{r})=-\Omega(\mathbf{r}) t_{1}
$$

and the magnetisation for the unit volume becomes

$$
M^{\perp}(\mathbf{r}, t)=M_{0}^{\perp}(\mathbf{r}) \exp \left\{\mathrm{i}\left(\omega_{0}+\Omega(\mathbf{r})\left(t-t_{1}\right)-\Omega(\mathbf{r}) t_{1}\right)\right\}
$$

As time continues to pass, the net phase shift with respect to resonance will decrease. Here it is shown that at a time $t_{2}$ such that $t_{2}=2 t_{1}$, the net phase acquired with respect to on resonance precession will return to zero, and the unit volume will be in phase with magnetisation precessing on resonance. This will hold true for any unit volume, and all unit volumes will rephase simultaneously to constructively interfere, forming a Hahn echo [72] as shown in Fig. 3.9, with phase evolution shown in Fig. 3.10. Upon rephasing of spins and constructive interference of the magnetisation from the unit volumes, the spins will again begin to dephase due to field inhomogeneities. As illustrated in Fig. 3.9, the rephasing of magnetisation from the unit volumes will be incomplete due to phase accumulation which is irreversible. This irreversible dephasing is the result of random processes such as diffusion within inhomogeneous magnetic fields. 

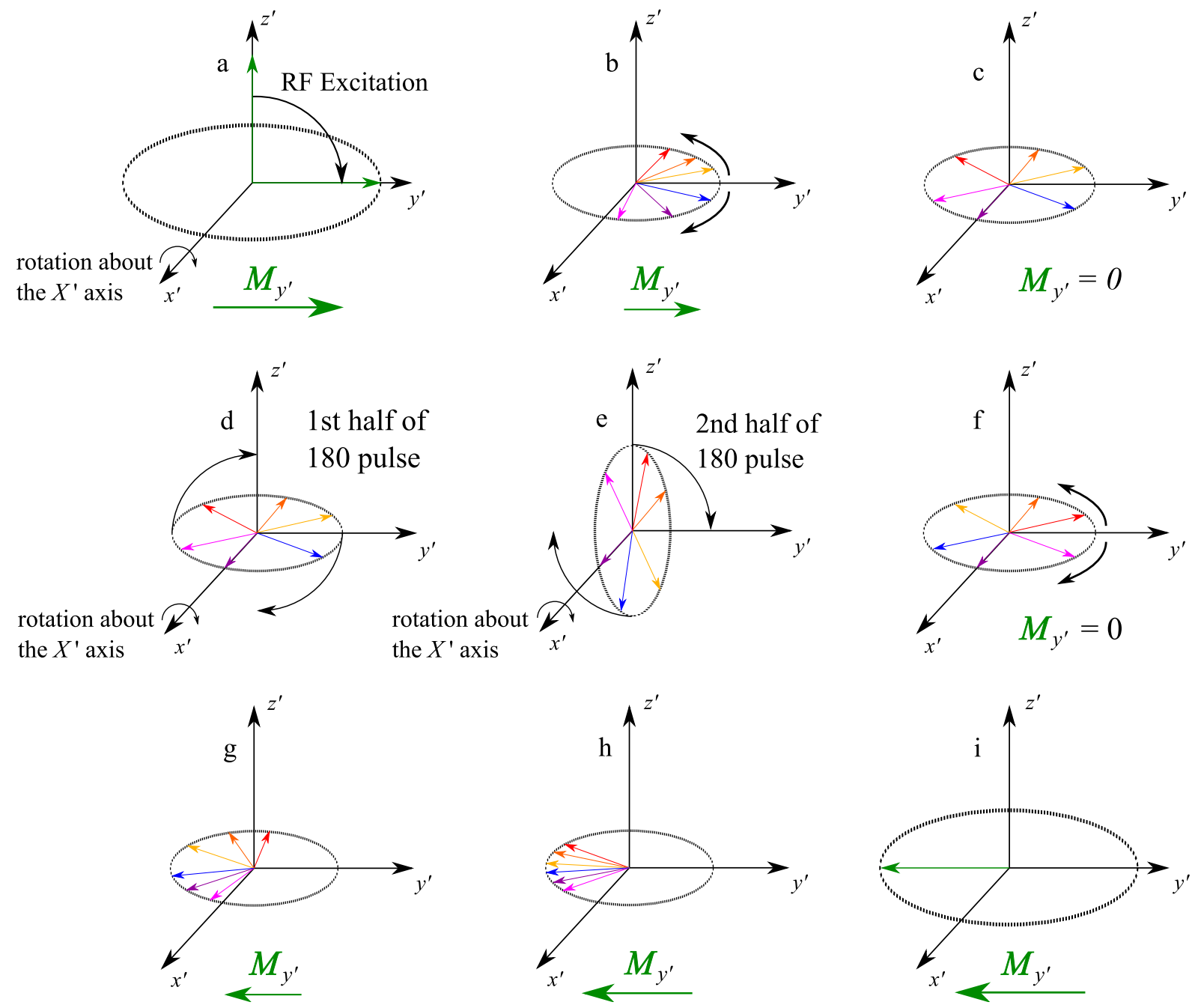

Figure 3.10: Time series phase evolution of the magnetisation of unit volumes at different positions in the NMR magnet. a) Excitation by a $90^{\circ} \mathrm{RF}$ pulse. b) Spins dephasing. c) Spins are completely dephased. d-e) $180^{\circ} \mathrm{RF}$ pulse is applied rotating the spins $180^{\circ}$ about the $x$ ' axis. f) Spins which were ahead in phase are now behind, vice versa, and phase accumulation due to fixed field inhomogeneities result in the spins beginning to rephase. g-h) Spins continue to rephase. i) Magnetisation from the unit volumes adds constructively to form a Hahn echo. 


\subsection{2 $\quad T_{2}$ Relaxation}

As discussed in the previous subsection on $T_{2^{*}}$ relaxation, the amplitude of a Hahn echo will fail to reach the original FID amplitude since irreversible dephasing of the spin ensemble will result in residual random phase accumulation. The irreversible dephasing resulting in transverse relaxation is referred to as $T_{2}$, or spin-spin relaxation.

The spin-spin relaxation is a driving force towards a thermal equilibrium amongst the spin ensemble. This process is a relaxation in which the spins dephase from one another such that their phases evolve towards equal distribution. This evolution towards a thermal equilibrium amongst the spins results in destructive interference between the associated magnetic moments of the unit volumes. The process of transverse relaxation may be expressed as

$$
\frac{d M^{\perp}}{d t}=-\frac{M^{\perp}}{T_{2}}
$$

assuming the substance composed by the nuclei is isotropic. Eq. 3.29 is satisfied with the relation

$$
M^{\perp}(t)=M^{\perp}(0) \exp \left\{-t / T_{2}\right\}
$$

To observe the irreversible spin-spin relaxation process, the spin ensemble may be repeatedly refocused to observe multiple echoes. Continuing on from the Hahn echo, a series of $180^{\circ} \mathrm{RF}$ pulses can be applied. This technique is known as a Carr Purcell Meiboom Gill (CPMG) sequence [73], and acts to refocus the spin ensemble multiple times, creating an echo train. Since only the reversible phase accumulation will be refocused, there will be a decreased spin coherence in the echo train as time evolves due to the accumulation of irreversible phase shifts throughout the spin ensemble. This loss of spin coherence results in a loss of constructive interference between the magnetic moments of each unit volume, and a decreasing net magnetic moment in the transverse plane. The time between the $90^{\circ}$ and $180^{\circ}$ pulses referred to as $t_{1}$ in subsection 3.2.1 is further denoted as $\tau$. In Fig. 3.11, a CPMG pulse sequence is shown where $180^{\circ}$ pulses are applied every $2 \tau$ after the first $180^{\circ}$ pulse to produce a series of echoes also spaced in time by $2 \tau$. To measure the $T_{2}$ time constant of the nuclei in their environment, the echo amplitudes, or the average 


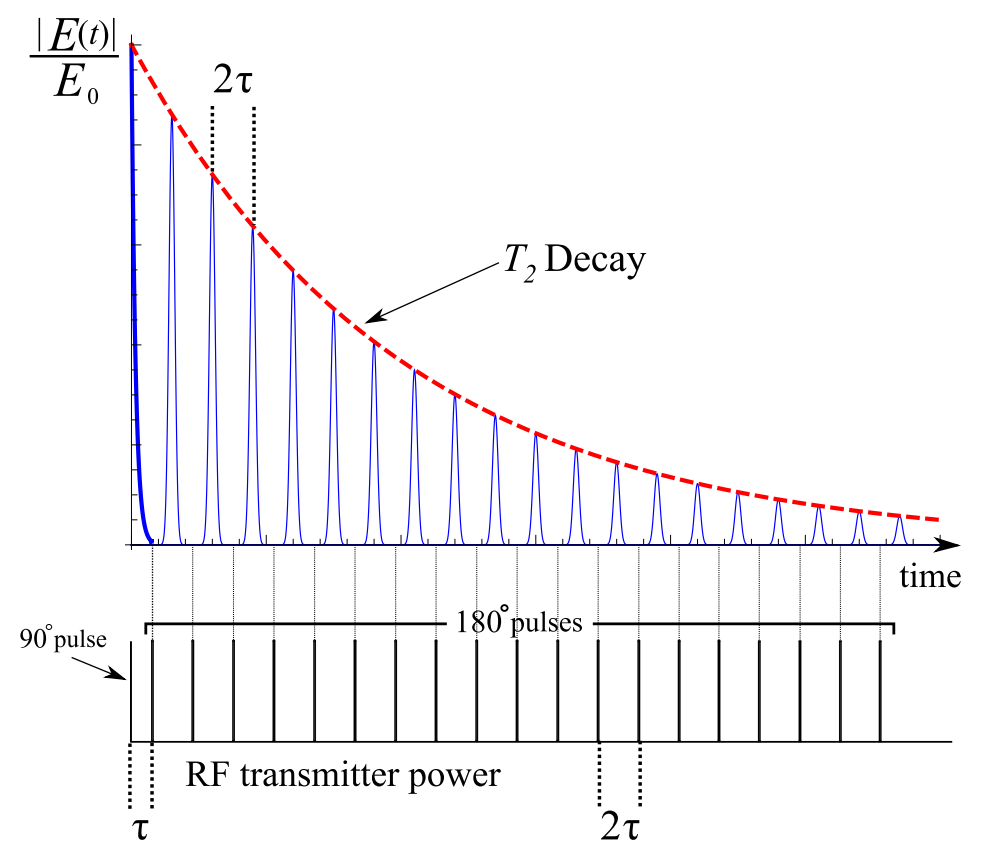

Figure 3.11: Top: Normalised RF receiver voltage showing initial FID and echo train. Bottom: RF transmitter power showing timing of RF pulses with respect to the RF receiver.

of a few points around each echo peak, are taken as the data points of interest. The fit of an exponential decay to these data points may be used to describe the $T_{2}$ of the system and the degree to which irreversible transverse relaxation is affecting the coherence of the nuclear spins.

\subsection{3 $T_{1}$ Relaxation}

The final form of relaxation which will be discussed is spin-lattice relaxation, or alternatively known as longitudinal or $T_{1}$ relaxation. The process of spin-lattice relaxation involves the exchange of energy between the nuclear spins and their surroundings which encompass the lattice with which they are in thermal equilibrium. As previously discussed, a nuclear ensemble will have a net spin and associated net magnetic moment equal to zero in the absence of a magnetic field. When an external magnetic field is applied, the Zeeman effect lifts the degeneracy of these spin states, creating an energy 
difference between them, making one energetically favourable. The result is a spin polarisation and associated longitudinal magnetisation $M^{\|}$which grows in time as depicted in Fig. 3.12. This process may be described by

$$
\frac{M^{\|}(t)}{d t}=-\frac{M^{\|}(t)-M_{e q}^{\|}}{T_{1}},
$$

where $M_{e q}^{\|}$is the magnetisation parallel to the $\mathbf{B}_{0}$ field at thermal equilibrium. This expression is satisfied by the relation

$$
M^{\|}(t)=M_{e q}^{\|}-\left[M_{e q}^{\|}-M^{\|}(0)\right] \exp \left\{-t / T_{1}\right\}
$$

where $M^{\|}(t)$ is the magnetisation along the direction of $\mathbf{B}_{0}$ as a function of time. If $\mathbf{B}_{0}$ is turned on at time $t=0$, the polarisation parallel to the $\mathbf{B}_{0}$ field can be expressed as

$$
M^{\|}(t)=M_{e q}^{\|}\left[1-\exp \left\{-t / T_{1}\right\}\right]
$$

and is depicted in Fig. 3.12. Since the thermal polarisation effects result in a net magnetisation aligned with $\mathbf{B}_{0}$, and $\mathbf{B}_{0}$ is conventionally represented as a static magnetic field pointing along the $z$-axis, or longitudinal direction, spin-lattice relaxation is also referred to as longitudinal relaxation. The characteristic time defining this relaxation process resulting in spin polarisation at thermal equilibrium is $T_{1}$.

After thermal polarisation has occurred and thermal equilibrium reached, tipping the magnetic moment of the nuclear ensemble away from $\mathbf{B}_{0}$ places the system in a nonequilibrium state. As soon as this occurs, the system will begin to progress back towards a state of thermal equilibrium until a constant, net magnetic moment of the nuclear ensemble is once again aligned with $\mathbf{B}_{0}$. This time dependent progression of magnetisation back towards thermal equilibrium will adhere to Eq. 3.32. The consequence of this progression is that of a time delay between excitation pulses for which the full magnetisation may be tipped back into the transverse plane. Usually a time delay of $5 \times T_{1}$ is used such that $99 \%$ of $M_{e q}^{\|}$has been recovered.

Measurement of the characteristic time $T_{1}$ of spin-lattice relaxation can be performed 


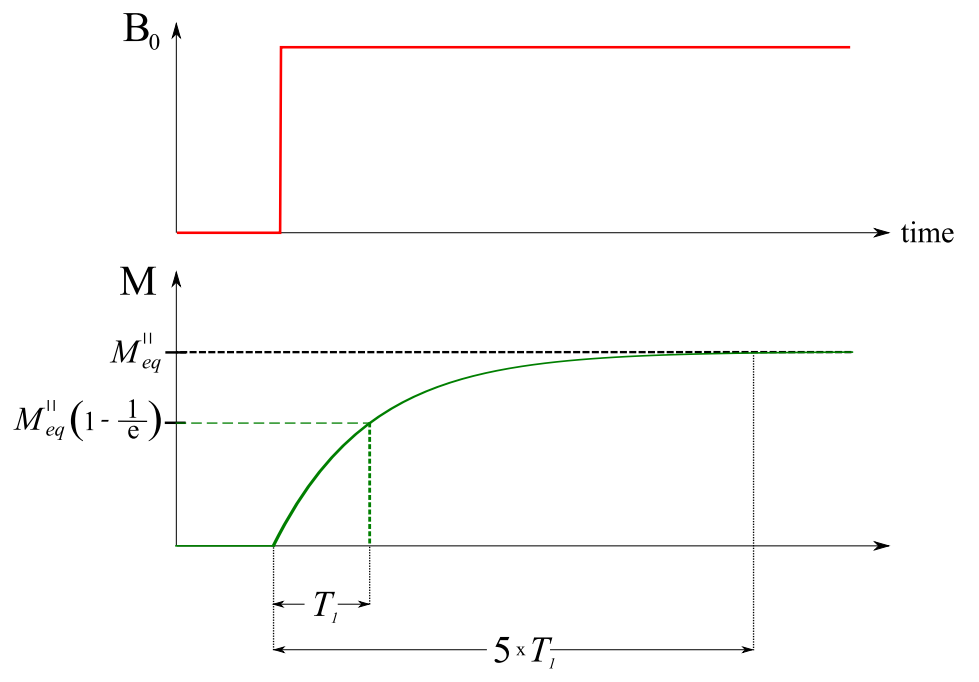

Figure 3.12: Top: A magnetic field $\mathbf{B}_{0}$ is turned on at some point in time. Bottom: The net magnetic moment parallel to $\mathbf{B}_{0}$ in a nuclear ensemble begins to increase in time upon application of the magnetic field.

through a variety of methods [74]. Here, a single method of $T_{1}$ measurement through a basic inversion recovery pulse sequence is presented to illustrate the recovery of net magnetisation in time after spin excitation due to spin-lattice relaxation.

The basic inversion recovery method of measuring $T_{1}$ begins by allowing the applied magnetic field to bring the nuclear ensemble into thermal equilibrium, producing a constant net magnetisation in the nuclear ensemble. After sufficient time for thermal polarisation has passed, a $180^{\circ} \mathrm{RF}$ pulse is applied to tip the magnetisation such that it opposes the applied $\mathbf{B}_{0}$ field. As time evolves, this magnetisation will undergo longitudinal relaxation, with the net spin and magnetisation building back up to its previous state in thermal equilibrium, as depicted in the top row of illustrations in Fig. 3.13. Before the magnetisation is allowed to fully recover and reach thermal equilibrium, a $90^{\circ} \mathrm{RF}$ pulse may be applied to tip this magnetisation into the transverse plane as shown in the bottom row of illustrations in Fig. 3.13, and the resulting FID is measured. Since it is the sign and amplitude of the longitudinal magnetisation which determines the sign and amplitude of the acquired FID, this allows the measurement of the longitudinal magnetisation at the time of the $90^{\circ} \mathrm{RF}$ recall pulse. After the FID is acquired, the system is allowed to return 

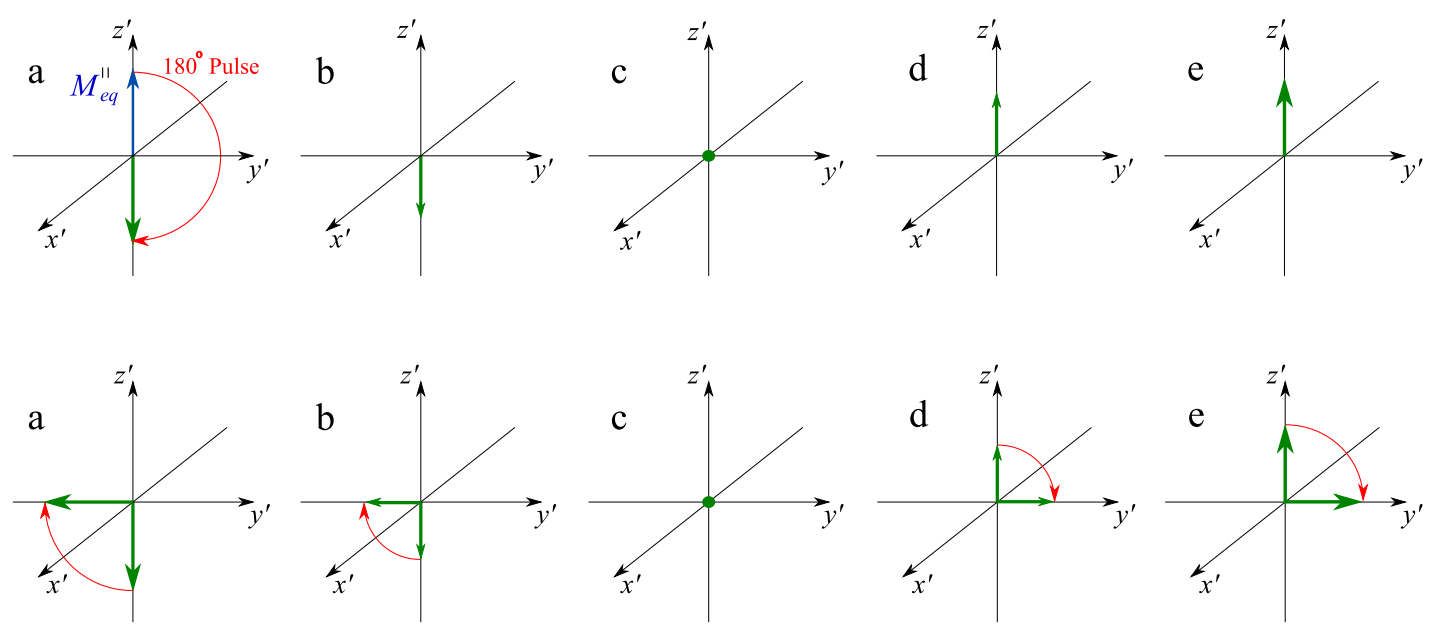

Figure 3.13: Top: a) The nuclear ensemble is in thermal equilibrium with net magnetisation lying along $B_{0}$ in the $\hat{z}$ direction. A $180^{\circ} \mathrm{RF}$ pulse is applied tipping the magnetisation such that it points in the $-\hat{z}$ direction. b-e) Time series of the build up of the net magnetisation in the $\hat{z}$ direction as spin-lattice relaxation works to bring the system back into thermal equilibrium. Bottom: The illustrations represent the same times as in the top row, but this time a $90^{\circ}$ pulse is applied to tip the magnetisation into the transverse plane allowing an FID to be measured.

to thermal equilibrium. Again, a $180^{\circ} \mathrm{RF}$ pulse is applied and a different recovery time elapses before the next $90^{\circ} \mathrm{RF}$ pulse is applied and resulting FID acquired. By varying the time between the $180^{\circ}$ and $90^{\circ} \mathrm{RF}$ pulses, the build up of longitudinal magnetisation along $\mathbf{B}_{0}$ will be recorded at different recovery times, resulting in a curve representing the spin polarisation process and the characteristic time for spin-lattice relaxation defined by $T_{1}$ (Fig. 3.14).

The time constant $T_{1}$ characterises a time restraint for the minimum repetition time of excitation pulses for a given longitudinal magnetisation recovery. For a series of experiments where a large parameter space must be sampled, or for signal averaging to increase signal to noise, $T_{1}$ defines the repetition time in such a series of experiments. To decrease total experimental times, creative pulse sequences are developed to sweep through as much of a parameter space as possible using a single excitation or small tip angle RF pulses. These sequences will minimise the number of excitation pulses or repetition times needed to sample the required parameter space, reducing or eliminating the portion of 


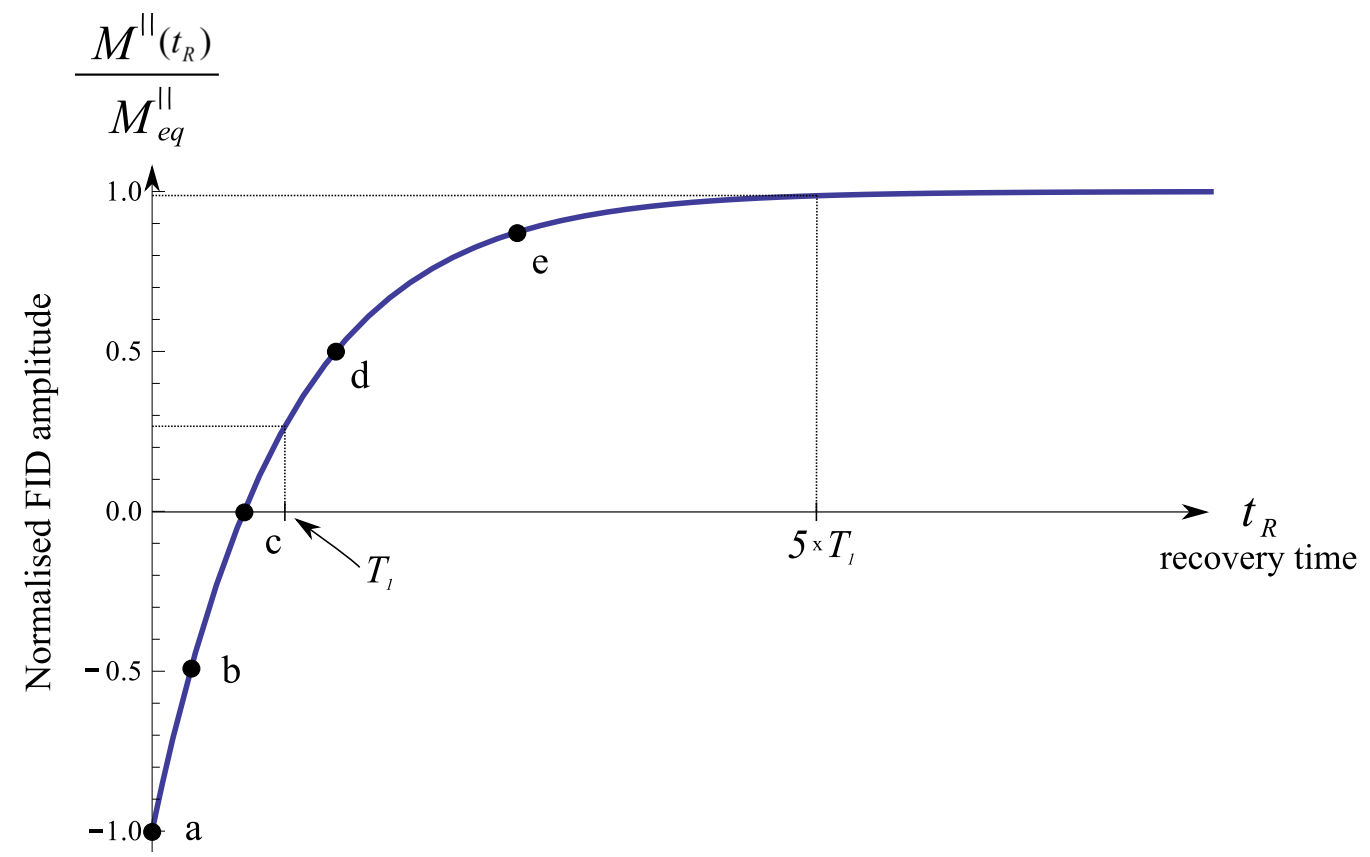

Figure 3.14: The FID amplitude is measured for the recovery times a-e shown in Fig. 3.13. The curve represents the build up of magnetisation due to the spin-lattice relaxation process which is directly proportional to the FID amplitude.

experimental time determined by $T_{1}$.

\subsubsection{Bloch Equations}

Taking the previously obtained results from this chapter about excitation and relaxation, Eq. 3.12, Eq. 3.29, and Eq. 3.31 can be combined in the rotating frame to arrive at the relations

$$
\begin{gathered}
\frac{\partial M_{x^{\prime}}}{\partial t}=\gamma M_{y}\left(B_{0}-\Omega / \gamma\right)-\frac{M_{x}}{T_{2}} \\
\frac{\partial M_{y^{\prime}}}{\partial t}=\gamma B_{1}-\gamma M_{x}\left(B_{0}-\Omega / \gamma\right)-\frac{M_{y}}{T_{2}},
\end{gathered}
$$

and

$$
\frac{\partial M_{z}}{\partial t}=-\gamma M_{y^{\prime}} B_{1}-\frac{M_{z}-M_{e q}^{z}}{T_{1}}
$$


where the z-axis is taken to lie along the direction of $\mathbf{B}_{0}, \Omega$ is the offset frequency from resonance due to additional magnetic fields, and $\mathbf{B}_{1}$ is taken to point along the $x^{\prime}$-axis in the rotating frame. These Bloch equations describe the evolution of spins, providing a method to predict and check behaviour in NMR experiments [68].

\subsection{Conclusions}

In this chapter a basic introduction to nuclear magnetic resonance has been presented. The concepts of nuclear spin and resulting net magnetisation in an NMR magnet have been discussed along with the use of RF pulses to excite and manipulate the polarised spin ensemble. Through these effects and methods NMR signals can be created, and provide the basis for the measurement techniques presented in the next two chapters.

The processes affecting NMR signal formation and decay have been briefly discussed, and relaxation effects responsible for net magnetisation and signal decay have been described. The longitudinal relaxation presents a limitation in repetition time for measurements requiring a series of experiments. This limitation is the obstacle which the new techniques developed in this research has sought to alleviate. Before presenting the new measurement techniques, relevant conventional techniques for spatial and displacement encoding are presented in the following chapter. 



\section{Chapter 4}

\section{Pulsed Magnetic Field Gradients in NMR}

\subsection{Introduction}

Magnetic field gradients are a key component of many NMR experiments. The spatial dependence of field strength results in a spatial dependence of the frequency at which the nuclei precess. This is the basis for spatial encoding and may be implemented in a variety of ways to extract information linked to space and time from the nuclei and the medium in which they are contained, allowing for a wide range of measurements. In this section an overview of the use of electromagnetic gradient coils will be given, along with their applications which are relevant to this research, with detailed discussion and uses of gradient coils in NMR provided elsewhere [66, 69, 75]. Of main interest are selective excitation of volumes, 1D imaging, and the measurement of displacements in time.

\subsection{Constant Magnetic Field Gradients}

Conventionally, the term 'gradient' in an NMR experiment refers to the use of an electromagnetic coil which produces a constant, non-zero magnetic gradient field in a single direction. To produce a gradient in an arbitrary direction, the superposition of three 
orthogonal gradients may be used such that

$$
B_{z}(\mathbf{r})=\left(B_{0}+\mathbf{g} \cdot \mathbf{r}\right)
$$

with

$$
\mathbf{g}=g_{1} \hat{x}+g_{2} \hat{y}+g_{3} \hat{z}
$$

where $g_{1}, g_{2}$, and $g_{3}$ represent the strength of the constant gradient fields produced by individual $x, y$, and $z$-gradient coils respectively.

The ability to turn the electromagnetic gradient coils on and off through the use of external power supplies allows the total magnetic field profile to be manipulated in time, and therefore the frequency of nuclear spin precession varied over space and time at will. Substituting Eq. 4.1 into Eq. 3.6, it is shown that

$$
\begin{aligned}
\omega_{L}(\mathbf{r}) & \left.=\gamma\left(B_{0}+\mathbf{g} \cdot \mathbf{r}\right)\right) \\
& =\omega_{0}+\Omega(\mathbf{r}),
\end{aligned}
$$

where $\omega_{0}$ is the resonant frequency without the applied gradient field, and $\Omega(\mathbf{r})$ is the spatially dependent offset frequency from resonance due to the applied gradient fields where inhomogeneities in the $\mathbf{B}_{0}$ field are neglected. In turn, this spatially dependent frequency gives rise to spatially dependent phase, where the amount of phase acquired during a gradient pulse of time $\delta$ will impart a phase shift off resonance phase of

$$
\phi(\mathbf{r})=\Omega(\mathbf{r}) \delta .
$$

For simplicity and illustrative purposes, the application of a lone $z$-gradient pulse is considered. Ignoring phase due to inhomogeneities and random effects, at some time after excitation all spins will still be in phase. Once the $z$-gradient is turned on, the Larmor frequency of these spins becomes spatially dependent on $z$, and will begin to accumulate phase relative to the spins precessing at $\omega_{0}$. The offset frequency is now dependent on position, and can be expressed as

$$
\Omega(z)=z \gamma g
$$



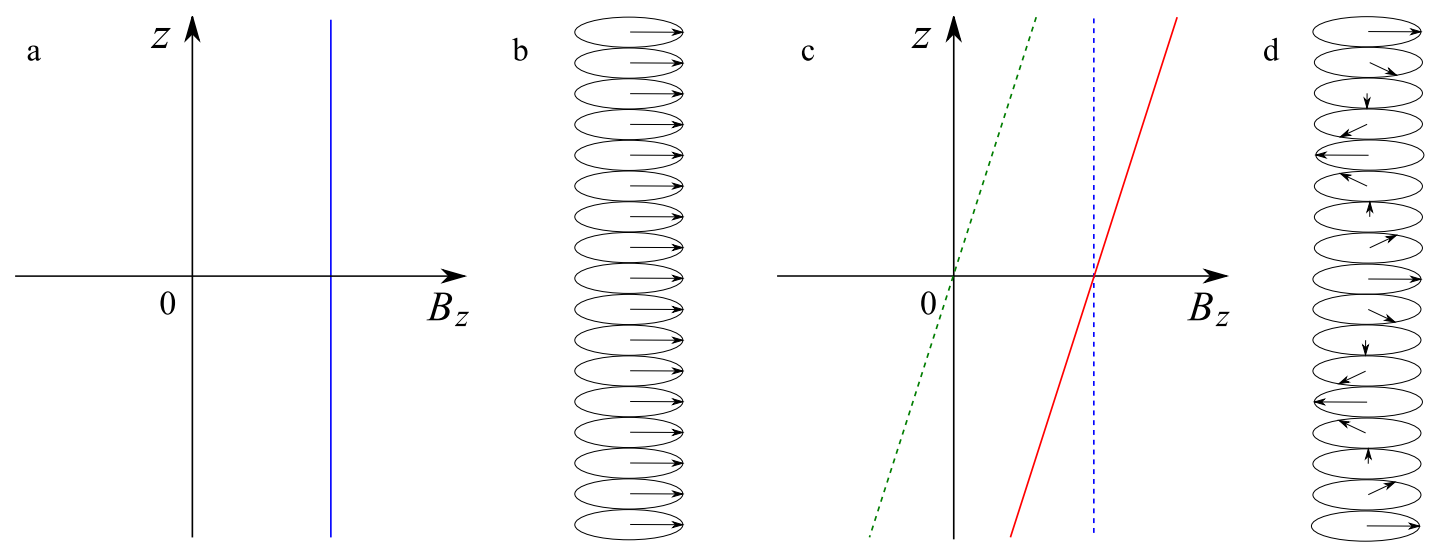

Figure 4.1: a: A homogeneous field $B_{0}=B_{z}$ is experienced by a spin ensemble. b) The spins are shown in a reference frame rotating at $\omega_{0}$ and no phase accumulation is present. c: A gradient field (dashed green) is added to the $B_{0}$ field (dashed blue) to give the total field shown in red. The Larmor frequency of the spins becomes spatially dependent on $z$. d: After some time $\delta$ has passed, spins will have accrued phase relative to on resonance spins precessing at $\omega_{0}$, according to their position in the gradient field.

The phase $\phi$ accumulated with respect to on resonance precession after a gradient pulse duration of $\delta$ is then expressed as

$$
\phi(z)=z \gamma g \delta
$$

This spatially dependent frequency and the phase it imparts is the basis for spatial encoding.

In this research three main techniques for spatial encoding are used: frequency dependence during signal acquisition for spatial resolution of signal, spatially selective excitation of the nuclei, and residual phase with respect to on resonance phase due to displacement of spins occurring between a pair of gradient pulses. 


\subsection{Spatial Resolution With Constant Magnetic Field Gradients}

\subsection{1 $k$-Space and the Read Gradient}

The application of a constant non-zero magnetic field gradient results in a Larmor frequency which is linearly dependent on position. During acquisition, if this magnetic gradient field is left on, the nuclei will precess at an offset frequency from resonance given by

$$
\Omega(\mathbf{r})=\gamma \mathbf{g} \cdot \mathbf{r}
$$

and the NMR signal will be a superposition of spatially dependent frequencies. The effect of this spatially dependent frequency during acquisition is that the signal acquired will be the inverse Fourier transform of the spin density in the direction of the applied gradient. Since this gradient is used to read out the spatial dependence of the signal, it is referred to as a 'read' gradient. To clarify the Fourier relationship between frequency and position, a reciprocal space, namely $k$-space, is used in the following signal analysis. The notion of $k$-space was introduced by Mansfield in 1973 [76], and is used to represent a reciprocal space vector

$$
\mathbf{k}(t)=\gamma \mathbf{g}(t)
$$

which possesses units of radians per meter. The phase evolution of the magnetisation is defined by substituting Eq. 4.7 into Eq. 3.25 to obtain

$$
M^{\perp}(\mathbf{r}, t)=M^{\perp}(\mathbf{r}, 0) \exp \{\mathrm{i} \gamma \mathbf{g}(\mathbf{t}) \cdot \mathbf{r}\}
$$

where inhomogeneities in the $\mathbf{B}_{0}$ field are neglected. Eq. 4.8 can then be substituted into Eq. 4.9 to obtain the phase evolution of the magnetisation in space using reciprocal space such that

$$
M^{\perp}(\mathbf{r}, t)=M^{\perp}(\mathbf{r}, 0) \exp \{\mathrm{ik} \cdot \mathbf{r}\}
$$


To obtain a Fourier relationship between position and $k$-space, the normalised signal is defined as

$$
E_{N}(t)=\int M^{\perp}(\mathbf{r}, t) / M^{\perp}(\mathbf{r}, 0) d \mathbf{r},
$$

and the normalised spin density as

$$
\rho(\mathbf{r})=M^{\perp}(\mathbf{r}, 0) / \int M^{\perp}(\mathbf{r}, 0) d \mathbf{r}
$$

to arrive at the relationships

$$
E_{N}(\mathbf{k})=\int \rho(\mathbf{r}) \exp \{\mathrm{ik} \cdot \mathbf{r}\} d \mathbf{r}
$$

and

$$
\rho(\mathbf{r})=(2 \pi)^{-1} \int E_{N}(\mathbf{k}) \exp \{-\mathrm{ik} \cdot \mathbf{r}\} d \mathbf{k}
$$

The read image takes advantage of the dependence of $\mathbf{k}$ on time by applying a nonzero magnetic field gradient pulse which is constant in amplitude across the acquisition window, yielding a linear relationship between $\mathbf{k}$ and $t$. To acquire a read image, a pulse sequence is used which is similar to a Hahn echo, but with the addition of read gradient pulses (Fig.4.2). Typically, a single gradient coil is used to apply a gradient field of constant strength along a single axis. The gradient pulses used first dephase the spin ensemble before the acquisition window, and then begin to rephase the ensemble at the beginning of the acquisition window, such that the rephasing of the spin ensemble due to $T_{2^{*}}$ effects and gradients occur simultaneously (Fig. 4.2). During the first gradient pulse used to dephase the spins, $k$-space is traversed in the negative direction. At the end of this first gradient pulse, the position in $k$-space is defined as

$$
k_{z}=-\gamma g \delta
$$

where $\delta$ is the duration of the first gradient pulse, a $z$-gradient pulse is applied, and the amplitude of this gradient is $g$. As the positive gradient pulse is applied at the beginning 


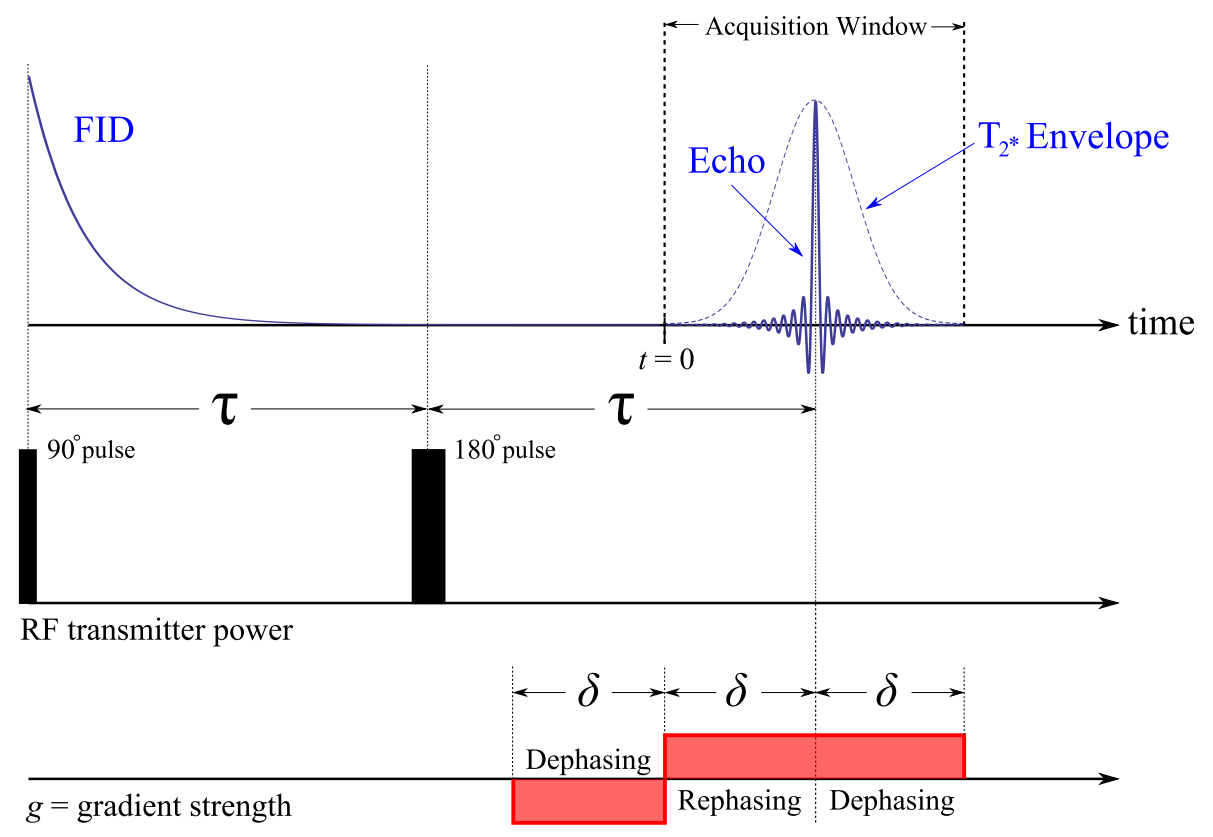

Figure 4.2: Pulse sequence for acquiring a read image. A Hahn echo is used with the addition of a negative gradient pulse to dephase the spins before the acquisition window, and a positive gradient to rephase the spins during the acquisition window. The gradient pulses are timed such that the Hahn echo and rephasing due to the read gradients occur simultaneously.

of the acquisition window, $k$-space is traversed in the positive direction such that

$$
k_{z}(t)=-\gamma g \delta+\gamma g t
$$

where time is measured from the beginning of the acquisition window. The duration of the acquisition window is equal to the duration of the second read gradient pulse, and the data for $k$-space is acquired for $-\gamma g \delta \leq k_{z} \leq \gamma g \delta$, giving a symmetric sampling of $k$-space data. When $t=\delta$, the spin ensemble forms an echo before being dephased by the remaining portion of the second read gradient pulse as shown in Fig. 4.3.

Since the echo shape contains information about the spin density distribution, parameters must be set such that the echo shape is not significantly affected by the $T_{2^{*}}$ amplitude envelope. For this reason, it is preferable that the rephasing of spins by the read gradient 


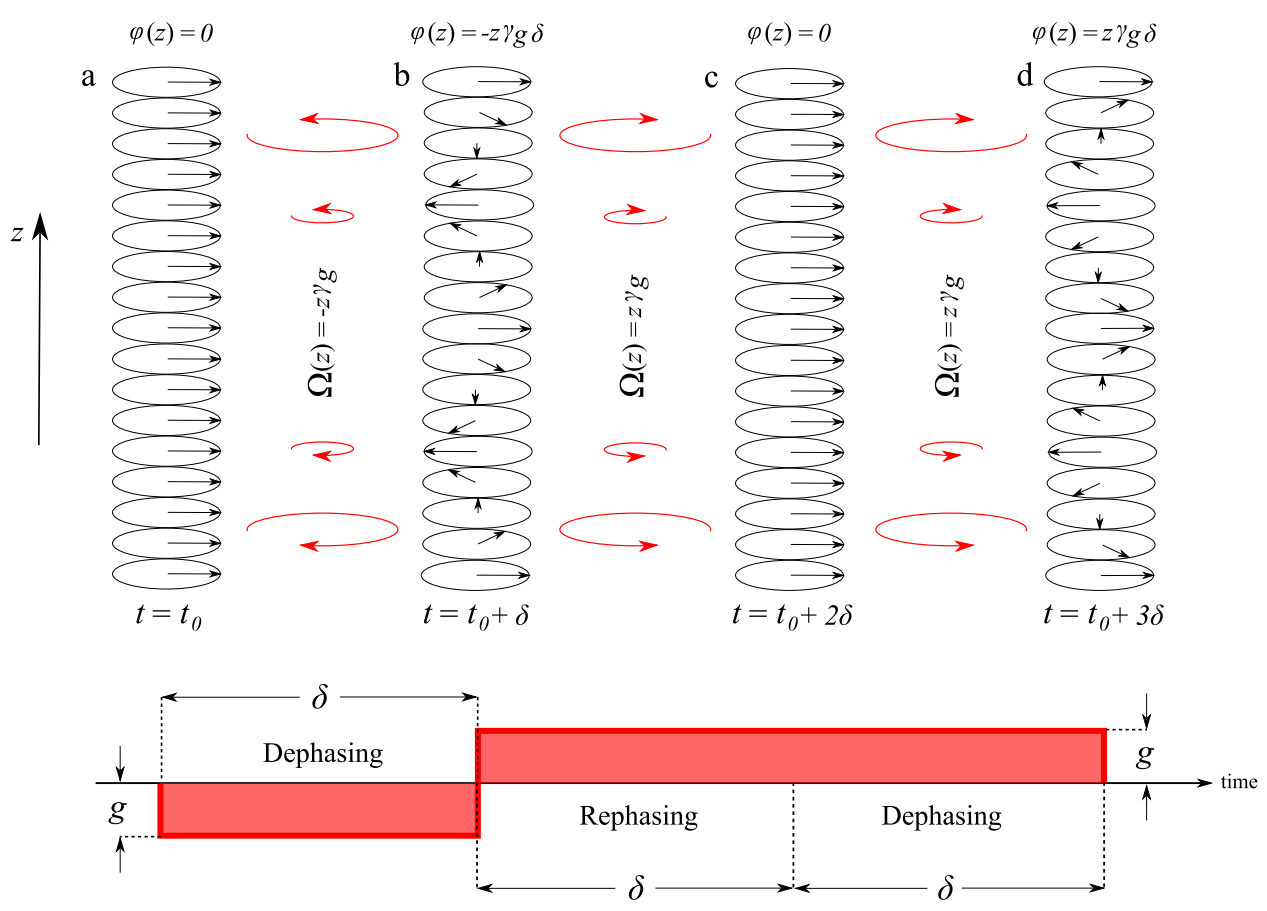

Figure 4.3: Top: Phase evolution along the $z$-axis due to read gradients in a read image pulse sequence. Bottom: Gradient strength as a function of time. a) The read gradient is turned on and spins begin to precess at their position dependent frequency. b) A phase accumulation of $\varphi(z)$ has occurred. The sign of the gradient is reversed and the spins begin to rephase as the sign of their offset frequency $\Omega(z)$ is reversed. c) Spins continue precessing at $\Omega(z)$, have rephased, and produce an echo. d) The spins have again dephased and the echo has vanished.

occurs at the same time as the rephasing due to $T_{2^{*}}$ effects, allowing the echo to occur under the least rapidly changing part of the envelope, with maximum signal envelope amplitude (Fig. 4.2). The amplitude of the gradient also plays a role as a higher gradient will cause a more abrupt refocusing of spins and an echo which is more compact in time, helping alleviate influence on its shape due to $T_{2^{*}}$ effects. This is an important consideration when information is obtained directly through the echo shape, an effect which is discussed in detail in section 5.3.

Other considerations when acquiring a read image are that of the dwell time, i.e. the time over which each data point is acquired, and the duration of the acquisition window. The 
dwell time will determine the frequency range sampled, and therefore the field of view, and the duration of the acquisition window will determine the resolution of the image. The linear relationship between $k_{z}$ and $t$ shown in Eq. 4.16 allows a simple conversion

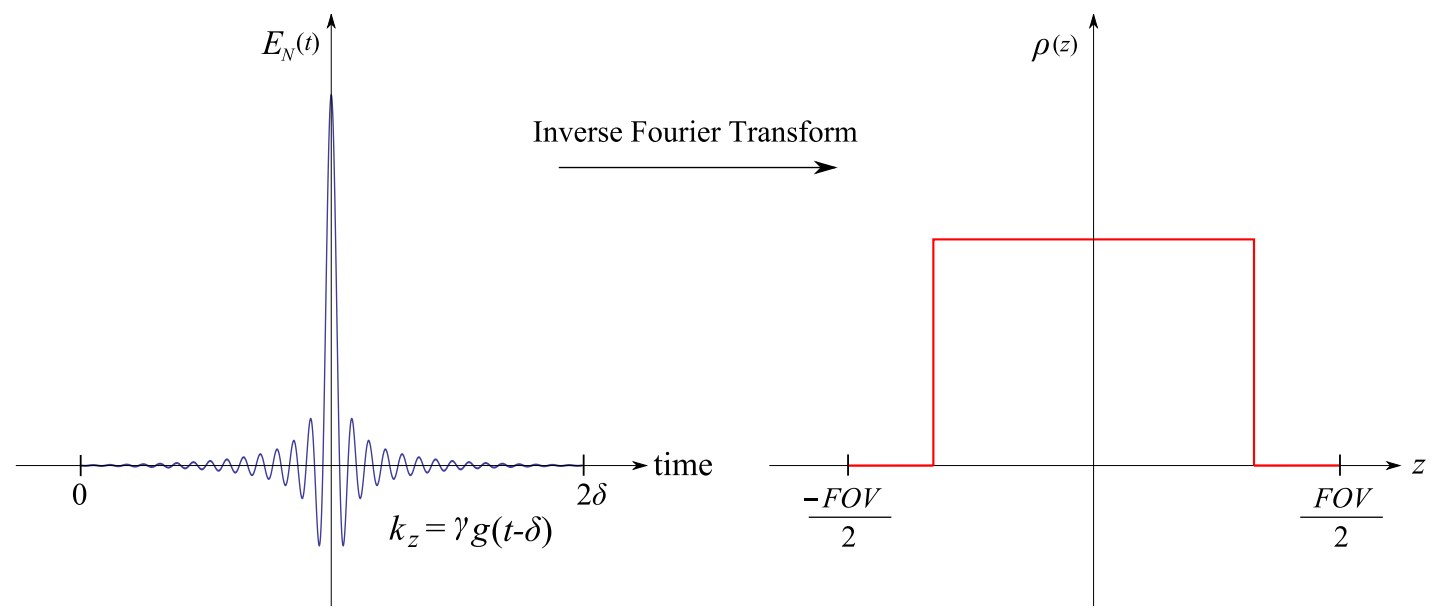

Figure 4.4: Left: An echo is acquired during the application of a read gradient. The echo acquired would be a series of points, but for illustrative purposes, is shown as a smooth curve. Right: An inverse Fourier transform is applied to the echo to produce a one dimensional image of the sample. Note, the one dimensional image shown is a rectangle, whose Fourier transform is a sinc function, the echo shape shown on the left.

factor of $\gamma g$ to be applied, transforming the signal as a function of time to the signal as a function of $k$-space. The inverse Fourier transform may then be applied to acquire the spin density $\rho(z)$ as shown in Fig. 4.4, and the field of view $(F O V)$ in real space calculated using the spacing of data points in $k$-space where

$$
F O V=2 \pi / \Delta k_{z}
$$

The read image is a crucial component of the methods developed in this research as it allows the extraction of spatial information using a single echo. This read image gives a spin density which is the integral over the entire cross section of the sample along the axis of the gradient. For some of the new experimental methods presented in this thesis, signal from only a thin slice of these cross sections is desired. The process of slice selection allows such regions to be selected, and is discussed in the next subsection. 


\subsubsection{Slice Selection}

In magnetic resonance imaging, the technique of slice selection allows an additional dimension of spatial resolution through selective excitation of a particular volume in the sample. Once again, a conventional gradient coil producing a constant, non-zero magnetic field is used for spatial encoding, this time with a 'soft' RF pulse to selectively excite or further manipulate a subset of the spin ensemble.

Hard and soft pulses differ mainly in the bandwidth they carry. The bandwidth of a pulse may be approximated by taking the inverse of the pulse duration, giving an estimation of the frequency range present in the pulse. A typical RF pulse, or 'hard' pulse, has a constant amplitude with a duration of $10-100 \mu$ s for a $90^{\circ}$ pulse. Hard pulses are considered anything with a duration such that their associated bandwidth is able to excite the entire nuclear ensemble, whose Larmor frequency may vary across the sample due to $B_{0}$ inhomogeneities, chemical shift, or gradient fields. The soft pulse has a longer duration, typically on the order of milliseconds, over which the amplitude is smaller and usually modulated. A soft pulse is composed of a relatively small bandwidth of frequencies compared to the hard pulse, allowing for excitation of a subset of the Larmor frequencies present. Pulses which are able to excite only a subset of the frequency distribution in the sample are considered soft.

A soft pulse $B_{1}(t)$ may be expressed as

$$
B_{1}(t)=A(t) \exp \left\{\mathrm{i} \omega_{0} t\right\}
$$

where $A(t)$ is the shaped amplitude envelope which modulates the amplitude of the central frequency component $\omega_{0}$. Taking the Fourier transform of $A(t)$,

$$
A(\Omega)=\int A(t) \exp \{\mathrm{i} \Omega t\} d t
$$

where $A(\Omega)$ describes the amplitude profile of the $B_{1}$ field as a function of offset frequency from resonance such that $\Omega=\omega-\omega_{0}$. This profile gives the bandwidth $\Delta \Omega$ of the soft pulse, which determines the window of frequencies about $\omega_{0}$ which will be affected by the soft pulse. 


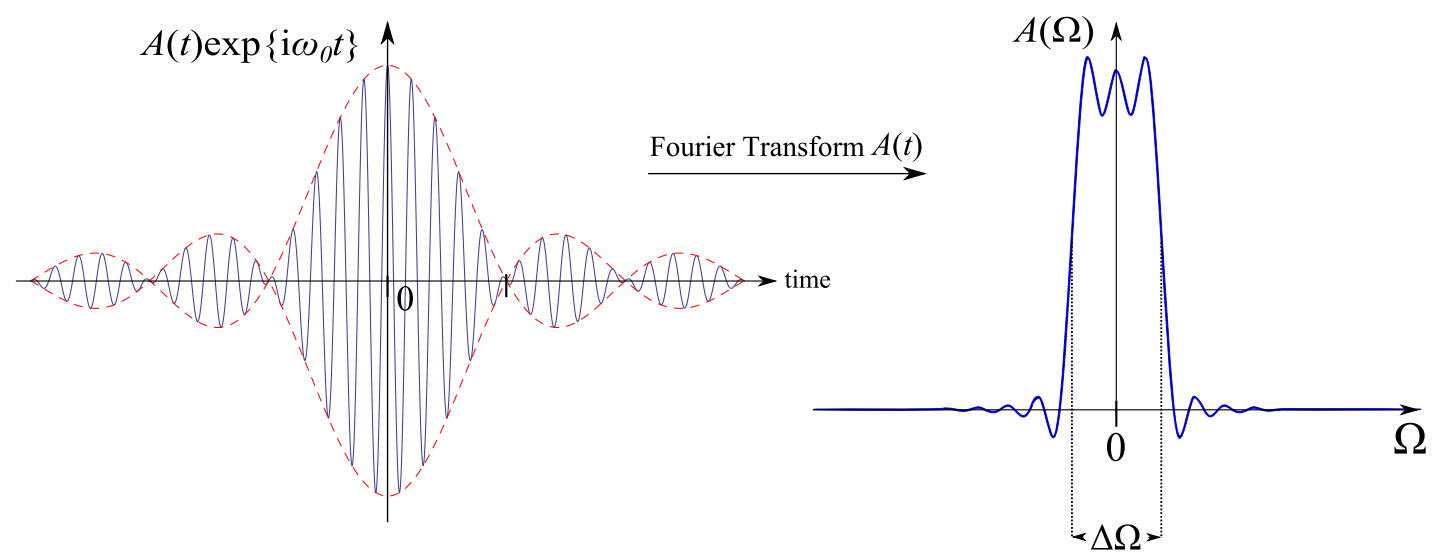

Figure 4.5: Left: A Sinc shaped soft RF pulse truncated to 3 lobes modulates the amplitude of the resonant frequency component $\omega_{0}$. For this example $A(t)$ is the Sinc function. Right: The associated bandwidth $\Delta \Omega$ of the Sinc pulse is obtained by performing a Fourier transform, showing the $B_{1}$ field strength $A(\Omega)$ as a function of offset frequency $\Omega$.

The example of a soft pulse composed of a truncated Sinc function is presented in this description, with the understanding that other soft pulse shapes may be used instead. The Sinc pulse and its Fourier transform are shown in Fig. 4.5. The amplitude modulation of $\omega_{0}$ by a Sinc function (left 4.5 ) results in the associated $B_{1}$ amplitude profile $A(\Omega)$ across a range of offset frequencies $\Omega$ (right 4.5). This illustrates the relationship of the shaped amplitude of the soft pulse and its duration to the bandwidth excited by the pulse.

The slice selection process can be performed by applying a soft RF pulse in the presence of a gradient field. The gradient field will affect the Larmor frequency distribution of the nuclei, making them linearly dependent on space for a constant gradient field. Considering a $z$-gradient which creates a magnetic field of

$$
B_{z}=z g
$$

where $g$ is the strength of the gradient in Tesla per meter, the Larmor frequency offset $\Omega$ from resonance will vary in space as

$$
\Omega(z)=z \gamma g
$$


This relationship between the Larmor frequency offset and position from Eq. 4.21 is

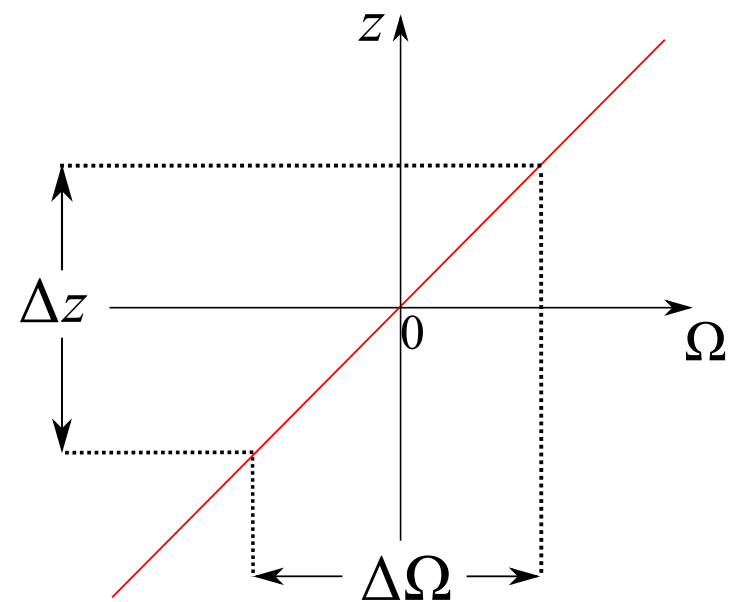

Figure 4.6: The relationship between position $z$ and offset frequency $\Omega$ from Eq. 4.21 is plotted in red. The bandwidth $\Delta \Omega$ of the soft pulse will only excite spins within the corresponding slice thickness $\Delta z$ as described in Eq. 4.22, and illustrated by the dashed lines.

illustrated in Fig. 4.6 in red. Since the soft pulse will only excite spins within its bandwidth, the excited slice thickness $\Delta z$ can be found by relating it to the bandwidth of the soft pulse $\Delta \Omega$ through Eq. 4.21 such that

$$
\Delta \Omega=\Delta z \gamma g
$$

This relationship, and the corresponding slice which is selectively excited by this process, is illustrated by the dashed lines in Fig. 4.6. 


\subsection{Measuring Displacement With Constant Mag- netic Field Gradients}

\subsubsection{The PGSE Experiment \& $q$-Space}

Another application of magnetic gradient fields in this research is that of gradient pulses used to sample $q$-space, the conjugate to displacement space, to measure self-diffusion and probability distributions for particle displacement. The notion of $q$-space differs from $k$ space in the sense that it allows measurement of displacement rather than position [66, 77]. A vector $\mathbf{q}$ can be defined in $q$-space where

$$
\mathbf{q}=\gamma \delta \mathbf{g}
$$

such that $\mathbf{q}$ is the conjugate to a displacement vector $\mathbf{r}-\mathbf{r}^{\prime}$, similar to how $\mathbf{k}$ is the conjugate to $\mathbf{r}$. Again, this spatial encoding is implemented through a pair of gradient pulses which act to dephase and rephase the spin ensemble, but are now separated by an observation time $\Delta$. A basic pulsed gradient spin echo (PGSE) pulse sequence uses a Hahn echo in conjunction with a pair of gradient pulses shown by $g$ in Fig. 4.7. The echo condition for this pulse sequence is defined as

$$
\int_{0}^{t} g^{*}(t) d t=0
$$

where $g^{*}(t)$ represents the effective gradient field which is time dependent (Fig. 4.7). The effective gradient differs from the applied gradient as it takes into account the history of the RF pulses and how they have altered the phase accumulation. For example, after a $90^{\circ} \mathrm{RF}$ pulse, when a positive gradient is applied, the value of $\mathbf{g}^{*}$ will simply be $\mathbf{g}$. If a $180^{\circ}$ pulse is then applied, another positive gradient pulse will result in the value of $\mathbf{g}^{*}$ as $-\mathbf{g}$ since the $180^{\circ}$ pulse changed the sign of the accumulated phase. Here it is noted that the even though Eq. 4.24 is fulfilled directly after the second gradient pulse shown in Fig. 4.7 , the echo does not occur until a time of $2 \tau$ since the gradients due to inhomogeneities 


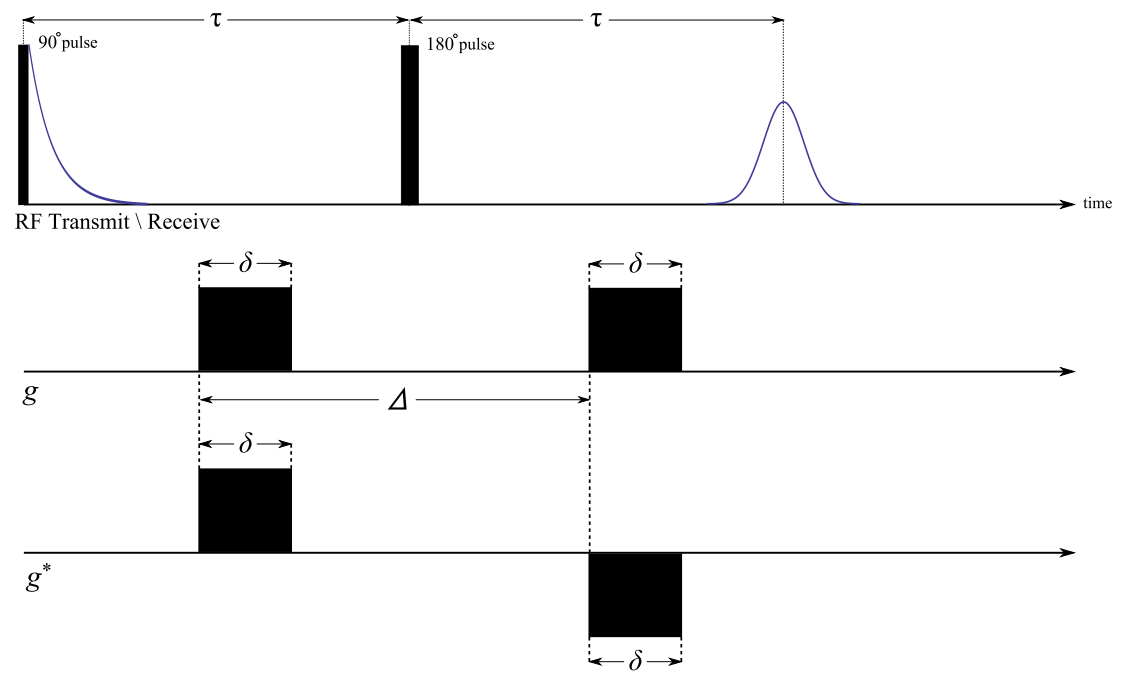

Figure 4.7: PGSE pulse sequence showing the effective gradient $g^{*}$.

in the static $B_{0}$ field must also be considered.

To sample $q$-space with the conventional PGSE pulse sequence shown in Fig. 4.7, a series of experiments is required. In such a series the observation time $\Delta$ is held constant, while the gradient strength, and therefore the parameter $\mathbf{q}$, is varied between experiments. This provides a method of acquiring $E(\mathbf{q})$, the echo signal as a function of $\mathbf{q}$. This data set yields information about the displacement of particles during the observation time $\Delta$, and can be used to measure the diffusion coefficient and the average propagator.

\subsubsection{Measuring Diffusion with PGSE}

The encoding for self-diffusion through gradient pulses was first shown by Stejskal and Tanner in 1965 [47], and has since developed into a complex measurement technique allowing the extraction of information relating to pore sizes $[33,54,55]$, surface to volume ratio and tortuosity [3, 52], as well as diffusion tensor imaging [78] in porous media. A data set which contains information for multiple $q$-space values or multiple observations times $\Delta$ can be used to extract the diffusion coefficient. Acquisition of such a data set with conventional PGSE experiments results in a series of experiments, and a total experimental time which is limited by the rate of thermal polarisation between experiments. 
To alleviate this time restriction, rapid measurement techniques have been developed for multidimensional diffusion ordered spectroscopy [79-82], and single-shot diffusion measurements which rely on chirped RF pulses which sweep over a large range of frequencies in the presence of gradients [79-83], second order magnetic fields [84], multiple excitations and multiple echoes [85-89], as well as multiple observation times [1, 22, 48, 90]. With the measurement technique presented in this research, the diffusion coefficient can be measured at fully correlated and well defined observation times such that all $q$-space measurements stem from the same initial point in time, a necessary condition for characterising systems which are evolving rapidly. All data is acquired using a single echo and a single excitation pulse, with the full relevant range of $q$-space sampled. This new method is discussed in detail in chapter 5. In the following, the experimental and theoretical considerations for the measurement of diffusion with the basic PGSE sequence using a constant magnetic field gradient is presented.

As discussed in the previous subsection, $q$-space data can be obtained through a series of PGSE experiments where the gradient strength is varied between experiments. This process is illustrated in Fig. 4.8. As shown by the series of three PGSE experiments, the echo amplitude decreases with increasing gradient strength. This is due to the diffusive attenuation experienced by incomplete rephasing of the spin ensemble from the second gradient pulse, an effect which is amplified for increasing gradient strength for a given observation time.

The measurement of signal attenuation is the effect through which the rate of diffusion is determined. After the first gradient pulse is applied, the spins are encoded with a phase dependent on their position in the gradient field. During the observation time $\Delta$, these spins will undergo random displacements in space. Since the phase imparted to spins due to the gradient field is dependent on their position, the phase imparted by the second gradient pulse may not cancel out the phase imparted by the first. As the displacements are random, the residual phase will also have a random distribution, resulting in an incomplete refocusing of the spins, and attenuation of the total signal acquired. In Fig. 4.9, the phase evolution of spins for the PGSE sequence shown in Fig. 4.8 is illustrated. For this illustration, a simplified case is presented where only two pairs of spins have swapped position along the $z$-axis. To determine the spin phase distributions, one can 

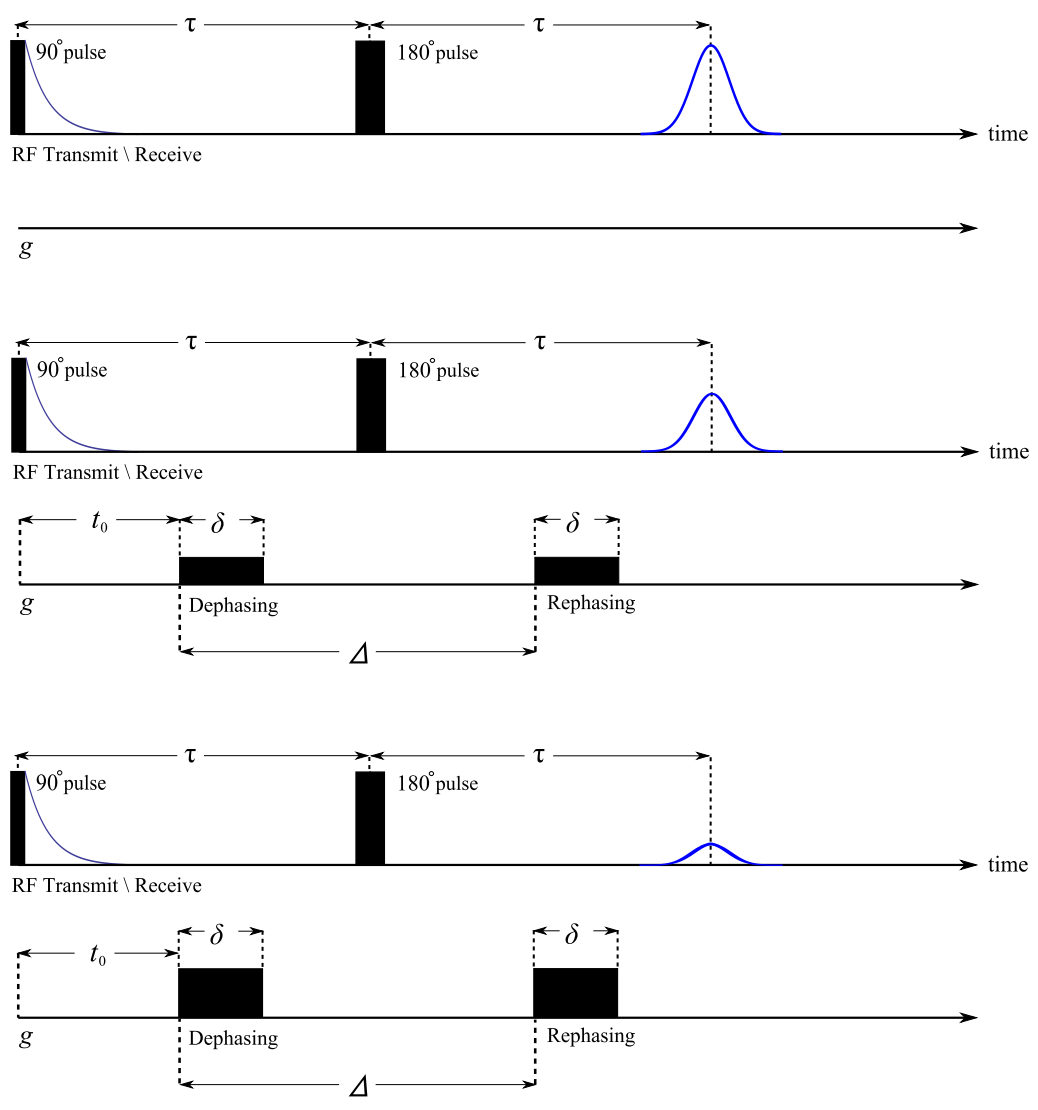

Figure 4.8: A series of three PGSE experiments. As the strength of the gradient pulses is increased, a decrease in echo amplitude is observed.

refer back to the Bloch equations, in particular Eq. 3.34 and Eq. 3.35. Terms accounting for displacement are now included to obtain

$$
\frac{\partial M_{x}}{\partial t}=\gamma M_{y}\left(B_{0}-\Omega / \gamma\right)-\frac{M_{x}}{T_{2}}+\nabla \cdot \mathbf{D} \nabla M_{x}-(\mathbf{v} \cdot \nabla) M_{x}
$$

and

$$
\frac{\partial M_{y}}{\partial t}=\gamma B_{1}-\gamma M_{x}\left(B_{0}-\Omega / \gamma\right)-\frac{M_{y}}{T_{2}}+\nabla \cdot \mathbf{D} \nabla M_{x}-(\mathbf{v} \cdot \nabla) M_{y} .
$$

Here, $\mathbf{D}$ is the diffusion tensor which, in this case, will be treated as a constant for isotropic, unrestricted diffusion, and $\mathbf{v}$ is the velocity of the spins accounting for flow. When considering the effect of the gradients, $B_{0}$ and $B_{1}$ are omitted, and only considering 

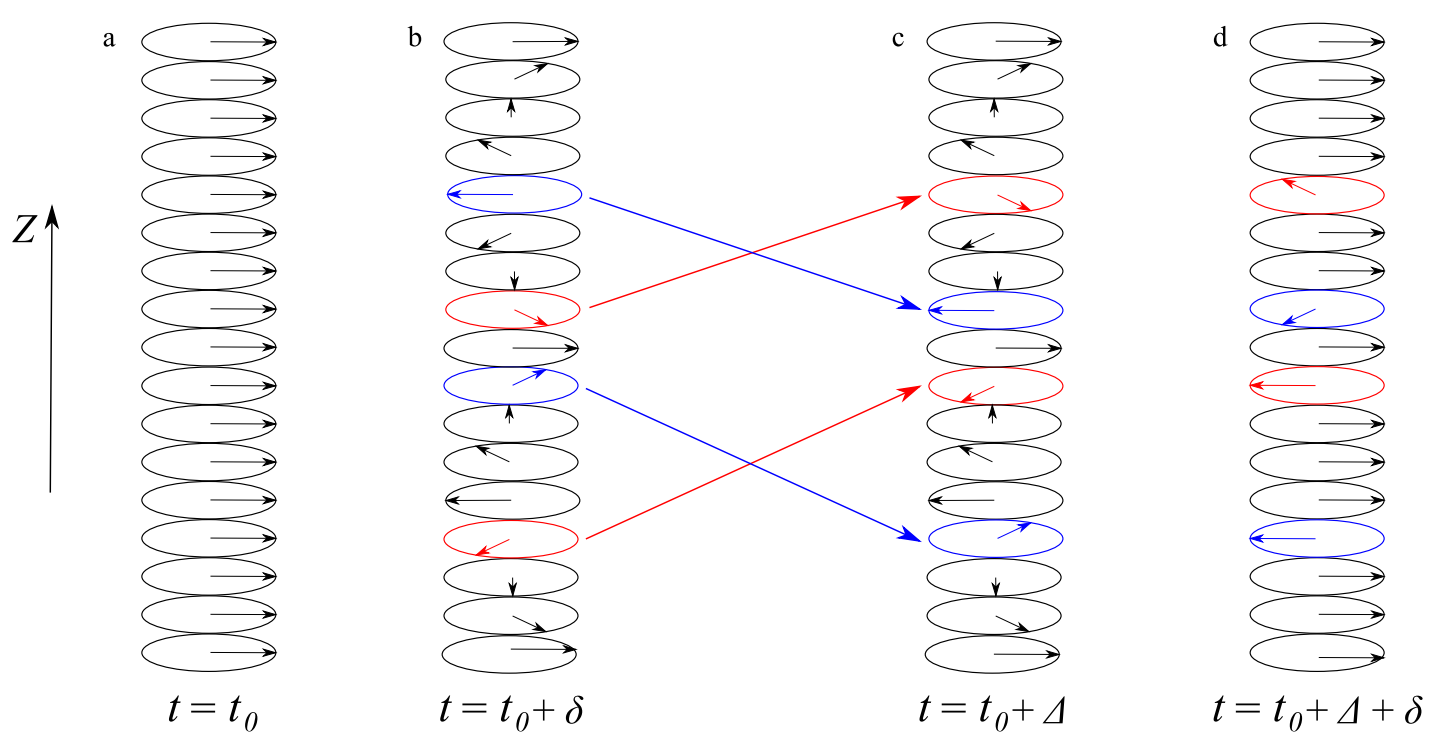

Figure 4.9: Phase evolution due to gradients in a PGSE pulse sequence. a) Spins along the z-axis are in phase before the first gradient pulse. b) After the first $z$-gradient pulse, phase dependent on position along the $z$-axis has been accumulated. c) After an encoding time $\Delta$, diffusion has occurred and spins have migrated to different positions. Here a simple case is presented where two pairs of spins have swapped position along the $z$-axis. d) After the second gradient pulse, there is incomplete rephasing of the spins as they are no longer in their original positions.

the offset frequency due to the gradients, $\mathbf{r} \cdot \mathbf{g}^{*}$ is substituted for $\Omega / \gamma$. Letting

$$
M^{\perp}=M_{x}+i M_{y}
$$

and by multiplying Eq. 4.26 by i and adding it to Eq. 4.25 , it is found that

$$
\frac{\partial M^{\perp}}{\partial t}=\left[-\mathrm{i} \gamma \mathbf{r} \cdot \mathbf{g}^{*}(t)-1 / T_{2}+D \nabla^{2}-(\mathbf{v} \cdot \nabla)\right] M^{\perp},
$$

where $\mathbf{g}^{*}$ is the effective gradient. This analysis continues by considering the case for no net flow such that $\mathbf{v}=0$. Omitting the term for velocity, a solution to Eq. 4.28 is given by

$$
M^{\perp}(\mathbf{r}, t)=A(t) \exp \left\{-\mathrm{i} \gamma \mathbf{r} \cdot \int_{0}^{t} \mathbf{g}^{*}\left(t^{\prime}\right) d t^{\prime}\right\} \exp \left\{t / T_{2}\right\}
$$


With the gradient pulses matched in strength and duration, the integral of $g^{*}(t)$ will be zero, and

$$
M^{\perp}(\mathbf{r}, t)=A(t) \exp \left\{t / T_{2}\right\}
$$

where $A(t)$ is a term which encompasses the effects of displacement on the NMR signal. In the case of diffusion with no net flow, $A(t)$ will be strictly real. Substituting Eq. 4.30 into Eq. 4.28, and considering the case for no net flow such that $\mathbf{v}=0$,

$$
\frac{\partial A(t)}{\partial t}=-D \gamma^{2}\left(\int_{0}^{t} \mathbf{g}^{*}\left(t^{\prime}\right) d t^{\prime}\right)^{2} A(t) .
$$

This may be integrated over time to quantify the effect of diffusion on the NMR echo signal as

$$
A(t)=\exp \left\{-D \gamma^{2} \int_{0}^{t}\left(\int_{0}^{t^{\prime}} \mathrm{g}^{*}\left(t^{\prime \prime}\right) d t^{\prime \prime}\right)^{2} d t^{\prime}\right\} .
$$

This term will always be $<1$ and will act to attenuate the NMR signal. Performing the integral of Eq. 4.32 over the basic PGSE pulse sequence, an expression for $A(t)$ can be obtained to provide a relationship between the experimental parameters in the pulse sequence and the NMR signal.

To determine $A(t)$, the timing of the gradient pulses and gradient amplitudes is defined as shown in Fig. 4.8, and the integral in Eq. 4.32 evaluated over the entire sequence. This analysis continues with the innermost integral of Eq. 4.32

$$
\int_{0}^{t^{\prime}} \mathbf{g}^{*}\left(t^{\prime \prime}\right) d t^{\prime \prime}
$$

The value of this integral is illustrated in Fig. 4.10. Here it is shown that only the time interval between $t_{0}$ and $t_{0}+\delta+\Delta$ will be non-zero, and the innermost integral of Eq. 4.32 may be evaluated in a piecewise fashion to obtain the relationship

$$
\begin{aligned}
\int_{0}^{t}\left(\int_{0}^{t^{\prime}} \mathbf{g}^{*}\left(t^{\prime \prime}\right) d t^{\prime \prime}\right)^{2} d t^{\prime}= & \int_{t_{0}}^{t_{0}+\delta}\left[g\left(t^{\prime}-t_{0}\right)\right]^{2} d t^{\prime}+\int_{t_{0}+\delta}^{t_{0}+\Delta}(g \delta)^{2} d t^{\prime} \\
& +\int_{t_{0}+\Delta}^{t_{0}+\Delta+\delta}\left[g \delta-g\left(t^{\prime}-t_{0}-\Delta\right)\right]^{2} d t^{\prime} .
\end{aligned}
$$




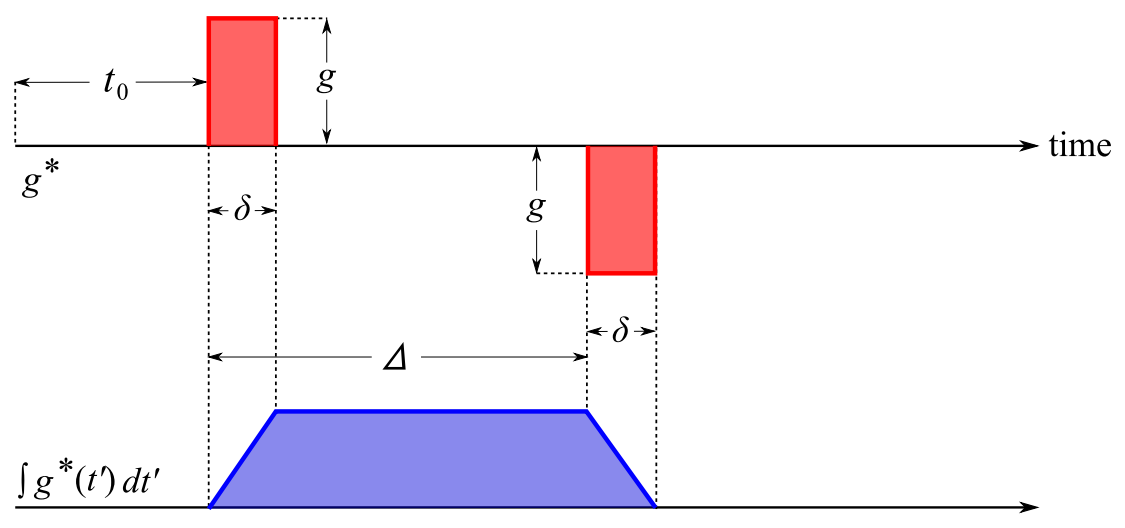

Figure 4.10: Integral of $\mathbf{g}^{*}$ over time for the PGSE pulse sequence shown in Fig. 4.8.

Performing the integrals in Eq. 4.34, and simplifying the result,

$$
\int_{0}^{t}\left(\int_{0}^{t^{\prime}} \mathbf{g}^{*}\left(t^{\prime \prime}\right) d t^{\prime \prime}\right)^{2} d t^{\prime}=g^{2} \delta^{2}(\Delta-\delta / 3) .
$$

Substituting Eq. 4.35 into Eq. 4.32, the expression for the modulation function for signal attenuation due to diffusion in a PGSE pulse sequence is obtained

$$
A\left(t_{0}+\delta+\Delta\right)=\exp \left\{-D \gamma^{2} g^{2} \delta^{2}(\Delta-\delta / 3)\right\}
$$

Eq. 4.29 may now be rewritten using the solution for $A(t)$ for a PGSE pulse sequence where $t>t_{0}+\delta+\Delta$, yielding

$$
M^{\perp}=\exp \left\{-D \gamma^{2} g^{2} \delta^{2}(\Delta-\delta / 3)\right\} \exp \left\{t / T_{2}\right\}
$$

Since the transverse magnetisation $M^{\perp}$ will be proportional to the NMR signal acquired, the echo signal amplitude can be rewritten as

$$
E(t) \propto \exp \left\{-D \gamma^{2} g^{2} \delta^{2}(\Delta-\delta / 3)\right\} \exp \left\{-2 \tau / T_{2}\right\}
$$


Keeping the echo time $2 \tau$ constant, and normalising the echo signal with respect to the echo obtained for $g=0$, the transverse relaxation term vanishes such that

$$
E_{N}=\exp \left\{-D \gamma^{2} g^{2} \delta^{2}(\Delta-\delta / 3)\right\}
$$

where $E_{N}$ is the normalised signal amplitude. Letting $q=\gamma g \delta$, a relationship between the signal as a function of $q$ and $\Delta$ is obtained for constant echo times.

$$
E_{N}(q, \Delta)=\exp \left\{-D q^{2}(\Delta-\delta / 3)\right\}
$$

Since $q$ and $\Delta$ are controlled experimental parameters and $\gamma$ is known, by varying $q$ and/or $\Delta$ over a series of scans and fitting the data to this model, the diffusion coefficient $D$ can be extracted.

\subsubsection{Shaped Gradient Pulses in PGSE Diffusion Measurement}

When varying parameters over a series of scans to measure the diffusion coefficient, it is desirable to collect multiple data points over a large range of attenuation to obtain a good fit. To cause significant diffusive attenuation, either long encoding times $\Delta$ or large gradient pulses resulting in large $q$ values may be used. Since $T_{2}$ effects will cause additional attenuation in the echo amplitude, long encoding times may not be desirable, but instead high gradient fields may be used while holding the observation time constant. When pulsing high currents through a gradient coil to produce strong gradients, to better control the shape of the pulse, ramping of current is a standard practice to prevent the amplifier from oscillating, overshooting, or lagging behind the desired pulse shape (Fig. 4.11). For the ramped pulses shown in Fig. 4.12, a ramping time of $\epsilon$ and duration at maximum amplitude $\delta-\epsilon$ results in a total area under the gradient pulse $g \delta$. Eq. 4.32 can then be evaluated to find a new expression for $A(t)$. With the ramp time taken into account, a similar solution is obtained, but now with a small correction term [91]. The relationship between the normalised signal and the diffusion coefficient can now be 


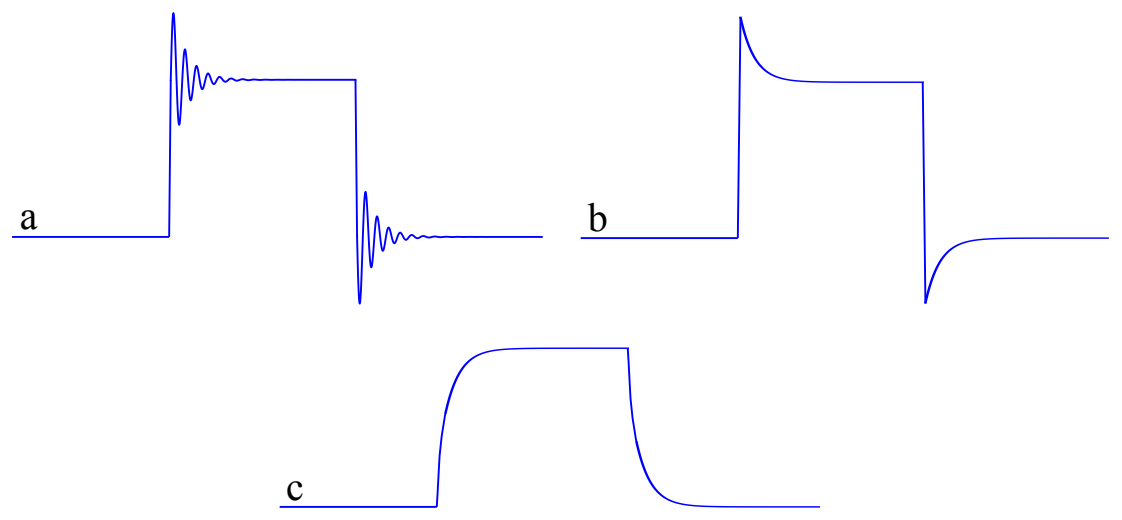

Figure 4.11: Potential responses from an amplifier in an attempt to produce a square pulse profile of high current. a) Amplifier oscillation. b) Amplifier overshooting the desired current level. c) Amplifier lagging behind the desired current level.

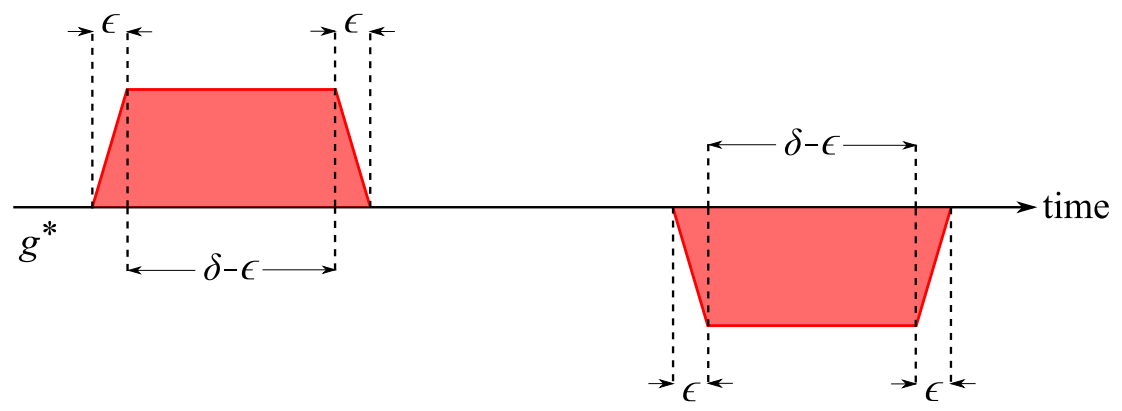

Figure 4.12: $g^{*}$ for ramped gradient pulses with ramp time $\epsilon$. Note $g^{*}$ is well defined, thus, $A(t)$ will also be well defined.

expressed as

$$
E_{N}(q, \Delta)=\exp \left\{-D q^{2}\left[\delta^{2}(\Delta-\delta / 3)+\epsilon^{3} / 30-\delta \epsilon^{2} / 6\right]\right\}
$$

In most cases the diffusion measurement is not significantly affected by these correction terms. Many other ramping shapes may be used, and again Eq. 4.32 may be evaluated to find $A(t)$, given the ramps and amplifier response are well defined [91]. 


\subsubsection{Measuring the Propagator with PGSE}

As discussed in chapter 2, the average propagator yields the probability distribution of particle displacement in space over some time interval. To relate the NMR signal to the average propagator, this analysis begins by accounting for the phase evolution of spins due to the basic PGSE sequence.

The phase factor of a spin at position $\mathbf{r}$ during a gradient pulse is given by $\exp \{\mathbf{i q} \cdot \mathbf{r}\}$, and upon application of a $180^{\circ}$ pulse, this phase factor will switch in sign. The weighted phase contribution from the first gradient pulse in the basic PGSE sequence can therefore be represented as

$$
\rho(\mathbf{r}) \exp \{-\mathbf{i q} \cdot \mathbf{r}\}
$$

The probability of a spin moving to a position $\mathbf{r}^{\prime}$ during the observation time $\Delta$, given it was at position $\mathbf{r}$ during the first gradient pulse, will be $P\left(\mathbf{r} \mid \mathbf{r}^{\prime}, \Delta\right)$. This results in a weighted phase factor due to the second gradient pulse of

$$
P\left(\mathbf{r} \mid \mathbf{r}^{\prime}, \Delta\right) \exp \left\{\mathbf{i q} \cdot \mathbf{r}^{\prime}\right\}
$$

The NMR signal due to spins starting at position $\mathbf{r}$ which have moved to position $\mathbf{r}^{\prime}$ during the observation time $\Delta$ will therefore be the product of Eq. 4.42 and Eq. 4.43. Since the NMR signal will be composed of all spins in the system, and all possible finishing positions $\mathbf{r}^{\prime}$ must be accounted for, the integrals over all starting and finishing positions must be performed. This yields a relationship of

$$
E(\mathbf{q})=\iint \rho(\mathbf{r}) \exp \{-\mathrm{iq} \cdot \mathbf{r}\} P\left(\mathbf{r} \mid \mathbf{r}^{\prime}, \Delta\right) \exp \left\{\mathrm{iq} \cdot \mathbf{r}^{\prime}\right\} d \mathbf{r} d \mathbf{r}^{\prime}
$$

The exponentials in this integral can be combined, and Eq. 4.44 rewritten as

$$
E(\mathbf{q})=\iint \rho(\mathbf{r}) P\left(\mathbf{r} \mid \mathbf{r}^{\prime}, \Delta\right) \exp \left\{\mathbf{i q} \cdot\left(\mathbf{r}^{\prime}-\mathbf{r}\right)\right\} d \mathbf{r} d \mathbf{r}^{\prime}
$$


Letting $\mathbf{R}=\mathbf{r}^{\prime}-\mathbf{r}$, Eq. 4.45 can be rewritten in terms of the starting position $\mathbf{r}$ and displacement $\mathbf{R}$ as

$$
E(\mathbf{q})=\iint \rho(\mathbf{r}) P(\mathbf{r} \mid \mathbf{r}+\mathbf{R}, \Delta) \exp \{\mathbf{i q} \cdot \mathbf{R}\} d \mathbf{r} d \mathbf{R}
$$

Using the definition for the average propagator $\bar{P}(\mathbf{R}, \Delta)=\int \rho(\mathbf{r}) P(\mathbf{r} \mid \mathbf{r}+\mathbf{R}, \Delta) d \mathbf{r}$, Eq. 4.46 is rewritten in single integral form as

$$
E(\mathbf{q})=\int \bar{P}(\mathbf{R}, \Delta) \exp \{\mathbf{i q} \cdot \mathbf{R}\} d \mathbf{R}
$$

This illustrates the Fourier relationship between the NMR signal $E(\mathbf{q})$ and the average propagator $\bar{P}(\mathbf{R}, \Delta)$. By acquiring the data set $E(\mathbf{q})$ and taking its Fourier transform, the average propagator is obtained.

\subsubsection{The Stimulated Echo and Pulsed Gradient Stimulated Echo}

The PGSTE sequence is important to this research as it can also be used to collect the data set $E(\mathbf{q}, \Delta)$. Similar to the PGSE sequence which is based on the Hahn echo, the pulsed gradient stimulated echo (PGSTE) is based on a stimulated echo. This subsection begins by describing the basic stimulated echo pulse sequence before incorporating gradient pulses to form a PGSTE.

The stimulated echo pulse sequence uses three $90^{\circ} \mathrm{RF}$ pulses to excite, store, and then recall magnetisation to form an echo. The first RF pulse tips the magnetisation into the transverse plane where it will dephase due to $T_{2^{*}}$ effects. The second RF pulse stores part of this magnetisation along the $z$-axis. After the storage pulse, the $M_{z}$ component may be stored for an extended period of time as it is immune to effects from transverse relaxation and gradient fields since it is not under precession, but is still subject to $T_{1}$ relaxation. The third $\mathrm{RF}$ pulse recalls the stored magnetisation into the transverse plane. Upon application of the third $90^{\circ}$ pulse, the previously stored magnetisation will begin to rephase, and an echo is formed. Since the echo will never form until the third RF pulse 
is applied, the echo is referred to as a stimulated echo.

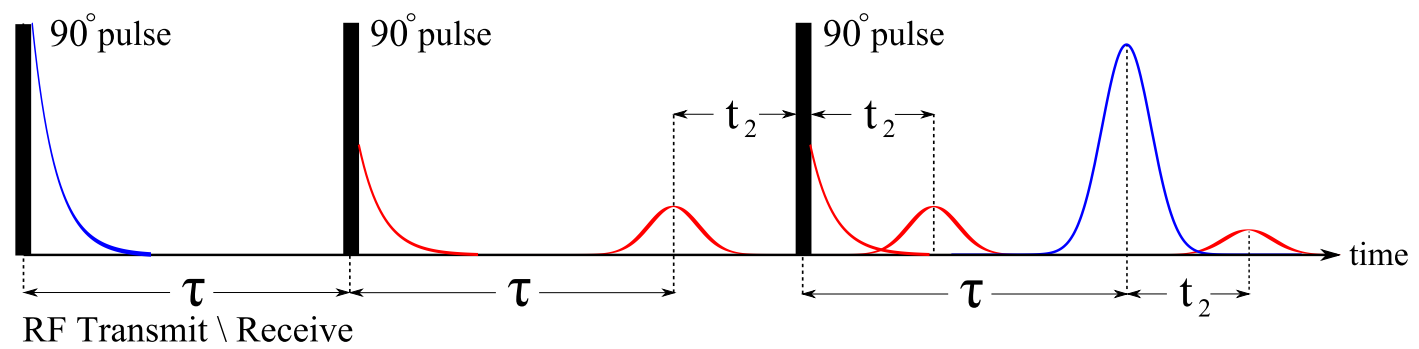

Figure 4.13: A typical stimulated echo pulse sequence. Ater the first RF excitation pulse and time $\tau$, a second RF pulse stores the magnetisation along the z-axis. After a storage period a third RF pulse recalls the stored magnetisation and the desired echo (blue) is formed as the spins rephase a time $\tau$ after the recall pulse. The undesired FIDs and echoes are shown in red. Here, only the amplitudes are shown for all signals formed in this sequence. Depending on the RF pulse phases, these signals may have positive and negative amplitudes, and can interfere constructively and destructively.

The three $90^{\circ}$ pulses used in the stimulated echo pulse sequence will cause multiple FIDs to form due to remaining or recovered magnetisation along the $z$-axis. The different portions of excited magnetisation resulting in these FIDs may also result in other echoes which are not desired. Although the RF pulses are designed to act strictly as $90^{\circ}$ pulses, they will have a small $180^{\circ}$ component to them (Fig. 4.13) which will cause portions of magnetisation in the transverse plane to refocus and form undesired echoes. The FIDs and echoes in this pulse sequence will have amplitudes which are positive and negative depending on the phases of the RF pulses used. In Fig. 4.13, only the magnitude of these signals are shown to simplify the illustration, but it should be noted that since some signals may be positive, and some negative, they may interfere constructively and destructively. By signal averaging over a series of experiments where the phases of the RF pulses are varied between experiments, the undesired signals can be averaged out to zero, and the stimulated echo signal acquired without interference.

The PGSTE pulse sequence uses the stimulated echo pulse sequence with the addition of a pair of gradient pulses to encode for displacement (Fig. 4.14). This is similar to the PGSE pulse sequence, but the formation of the stimulated echo in the PGSTE sequence differs significantly from the rephasing of spins in a PGSE sequence. To visualise this, 
the phase imparted only by gradients in a PGSTE sequence is considered, with the understanding that dephasing and rephasing of spins due to $T_{2^{*}}$ effects will follow the same analysis.

The evolution of the magnetisation due to a typical PGSTE pulse sequence is shown in Fig. 4.14. After the first $z$-gradient pulse has imparted spatially dependent phase along the $z$-axis, the storage pulse rotates the transverse magnetisation component perpendicular to the RF pulse onto the $z$-axis, while the transverse magnetisation component parallel to the RF pulse remains in the transverse plane.

After the storage RF pulse, another gradient pulse is applied which is called a homo-spoil gradient, depicted in blue in Fig. 4.14. The purpose of this pulse is to further dephase the magnetisation remaining in the transverse plane such that it does not interfere with the stimulated echo signal. This process results in a loss of half the available phase information since only the phase information from the stored magnetisation will be retained. To acquire the full phase information with a PGSTE, two experiments are required for each $\mathbf{q}$ or $\Delta$ value where the phase of the storage pulse is changed by $90^{\circ}$ in between the two experiments. This is an important factor in this research because the new techniques presented in chapter 5 are aimed at performing single-shot measurements. In the case of diffusion no phase information is required, and the PGSTE remains suitable for singleshot diffusion measurements. For flow measurements all phase information is required. Since the PGSTE is unable to acquire all the phase information in a single scan, it is an unsuitable technique for single-shot flow measurements.

Following the homo-spoil gradient pulse comes the third $90^{\circ}$ pulse, or recall pulse, which rotates the magnetisation stored along the $z$-axis back into the transverse plane. Here, the spins will rephase in a slightly different manner compared to the PGSE sequence since the phase is not continuously distributed along the $z$-axis. In this case, there will only be two available phases for the spins which are recalled, either 0 or $\pi$. This results in alternating regions of phase with length $\pi / \gamma \delta g$. The final gradient pulse acts dually as a spoiler gradient to dephase the undesired FID forming from the recall RF pulse, and also as a rephasing gradient for the stored magnetisation which is tipped back into the transverse plane to form the stimulated echo. As long as this gradient pulse is matched in strength and duration with the first gradient pulse, the regions of phase will re-align 

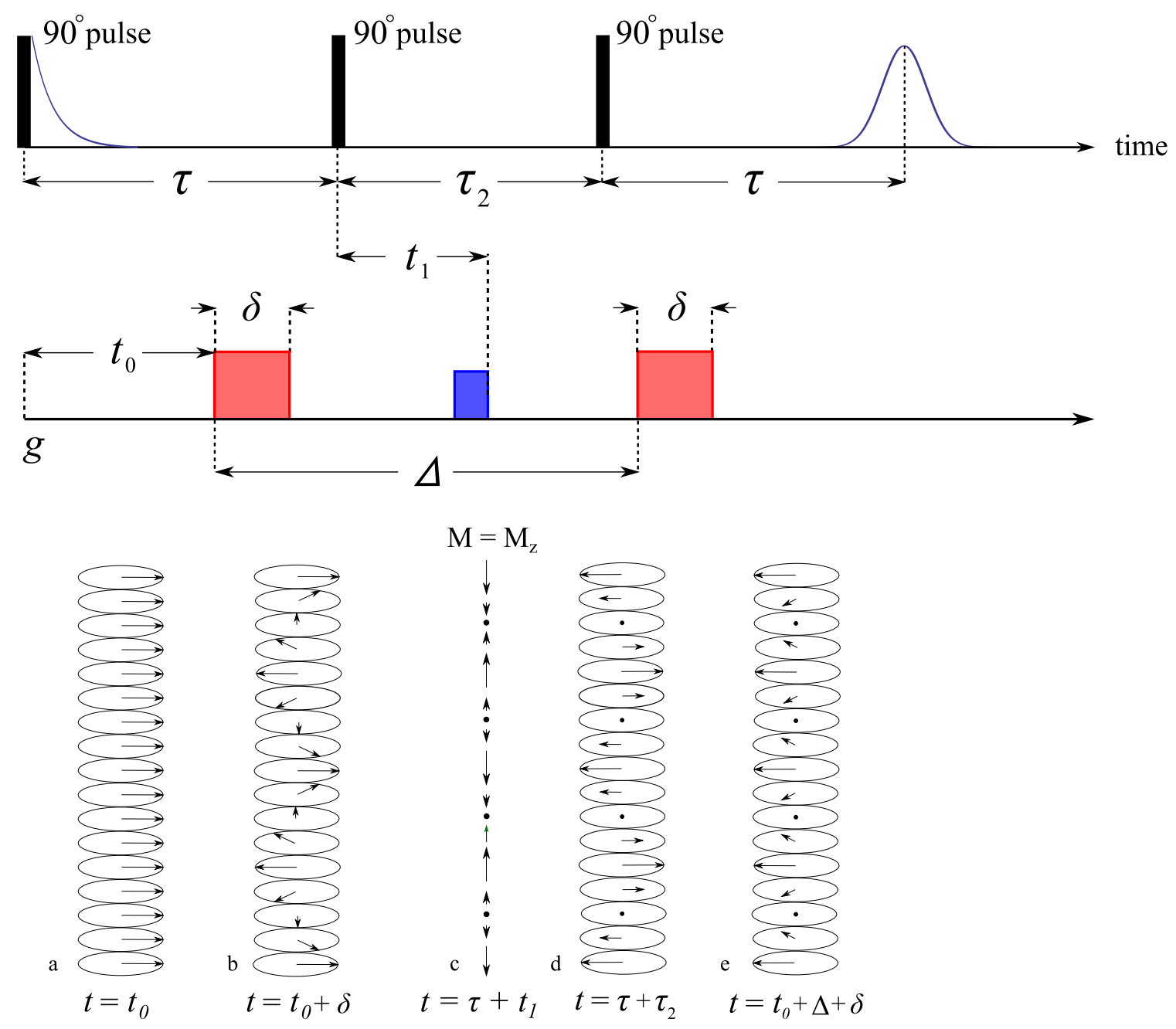

Figure 4.14: PGSTE pulse sequence and resulting magnetisation evolution. a) In phase spins along the $z$-axis at the beginning of the first $z$-gradient pulse. b) Spins have acquired phase $\phi(z)$ after the first z-gradient pulse. c) After the second RF pulse rotates the magnetisation, the homo-spoil gradient pulse is applied, and only the magnetisation lying along the $z$ axis remains. This magnetisation is immune to gradient fields and $T_{2}$ relaxation while it is stored. d) The third RF pulse rotates the stored magnetisation back into the transverse plane. e) The second gradient pulse rephases the magnetisation. At time $\tau$ after the third RF pulse, the $T_{2^{*}}$ effects are undone and a partial echo is formed. 
with one another as shown in Fig. 4.14, constructively interfering to form an echo. The optimal level of constructive interference which may be achieved for this pulse sequence is a factor of $1 / \sqrt{2}$ of the magnetisation which was stored along the $z$-axis. Along with the storage pulse which stores a factor of $1 / \sqrt{2}$ of the originally excited magnetisation, the stimulated echo suffers an inherent reduction in echo amplitude of at least $1 / 2$. Not shown in Fig. 4.14 is the element of diffusion, which will also contribute to signal attenuation, enabling measurement of the diffusion coefficient.

\subsubsection{Difftrain}

The DiffTrain [90] pulse sequence (Fig. 4.15) is based on the PGSTE pulse sequence, but with a fraction of the stored magnetisation recalled at various points in time to form a train of echoes. To recall a fraction of the stored magnetisation, an RF pulse of tip angle $\alpha_{1}<90^{\circ}$ is used to partially tip the stored magnetisation back into the transverse plane such that the fraction $\cos \left\{\alpha_{1}\right\}$ of the stored magnetisation is left along the $z$-axis. The recalled fraction $\sin \left\{\alpha_{1}\right\}$ of the magnetisation is subject to the rephasing diffusion gradient pulse, and will form an echo, while the stored component does not experience any phase accumulation as it is not under precession. After the echo is acquired, another recall pulse $\alpha_{2}<90^{\circ}$ is applied and the process repeated. Given an initial stored magnetisation of $M_{0}^{S T}$, the magnetisation tipped into the transverse plane by the second recall pulse is $M_{0}^{S T} \cos \left\{\alpha_{1}\right\} \sin \left\{\alpha_{2}\right\}$, and the magnetisation left in storage along the $z$-axis is $M_{0}^{S T} \cos \left\{\alpha_{1}\right\} \cos \left\{\alpha_{2}\right\}$. This analysis can be repeated to determine the amount of recalled and stored magnetisation remaining after each recall RF pulse.

The Difftrain pulse sequence is used for measurement of the diffusion coefficient in this research. This pulse sequence utilises a single value of $q$ and forms multiple stimulated echoes, each corresponding to a different observation time $\Delta$ to acquire a data set $E(\mathbf{q}, \Delta)$. The echoes formed from Difftrain will experience different amounts of $T_{1}$ relaxation during the varying storage times, and may also vary in the amount of magnetisation recalled for each echo depending on the RF power used for each recall pulse. This can result in varying echo amplitudes throughout the echo train in the absence of diffusion gradient pulses. Since diffusive attenuation must be measured with respect to the signal ampli- 


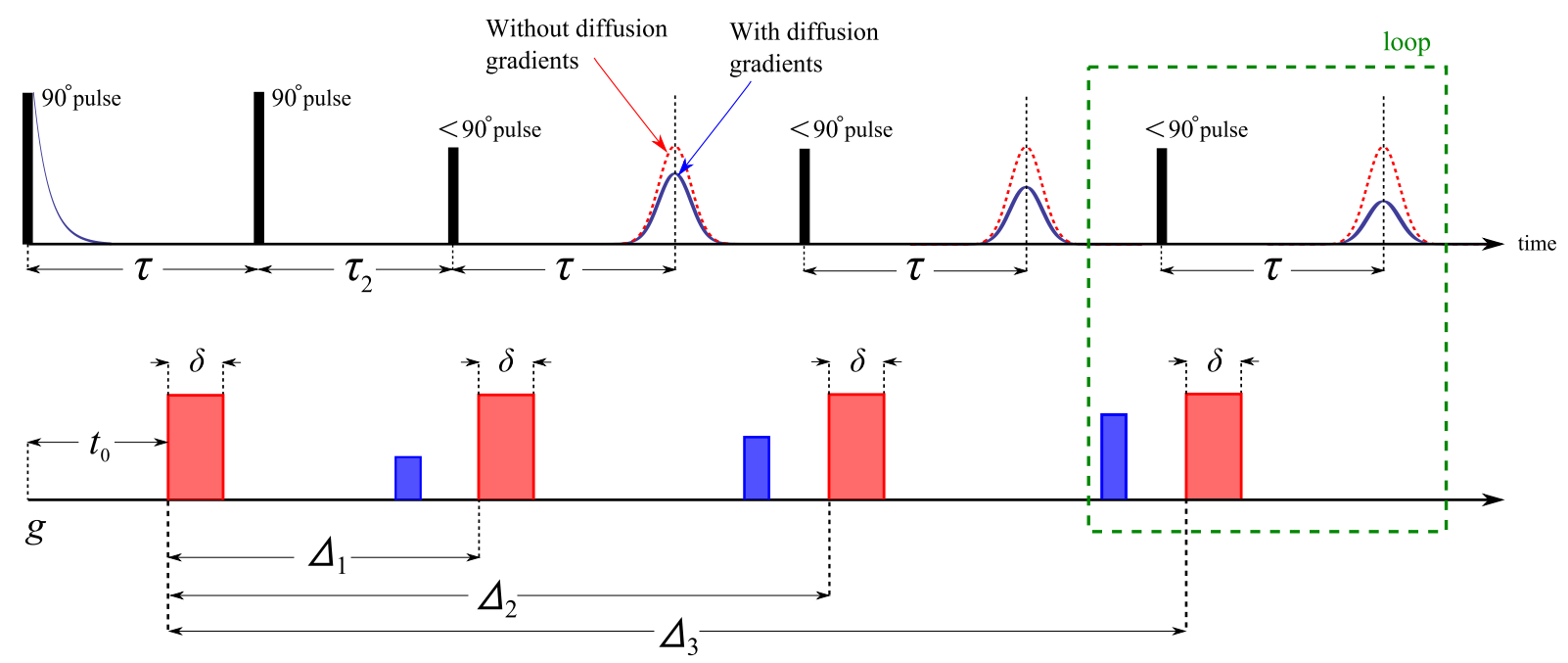

Figure 4.15: Difftrain pulse sequence forming multiple stimulated echoes. Diffusion gradient pulses are shown in red, and homo-spoil gradient pulses shown in blue. The amplitude of the homo-spoil gradient pulses are varied to avoid refocusing of previously recalled magnetisation.

tude in the absence of diffusion gradient pulses, a reference scan is required to perform a diffusion measurement. By acquiring the echoes without the application of diffusion gradient pulses $E(0, \Delta)$, the echo amplitudes with the application of diffusion gradient pulses $E(\mathbf{q}, \Delta)$ can be normalised such that

$$
E_{N}(\mathbf{q}, \Delta)=\frac{E(\mathbf{q}, \Delta)}{E(0, \Delta)}
$$

This normalised data set provides the correct measure of diffusive attenuation experienced at each observation time, and can therefore be related to the diffusion coefficient through Eq. 4.40.

When using the pulse sequence DiffTrain, multiple homo-spoil gradients must be used to dephase magnetisation remaining in the transverse plane before a new stimulated echo is formed. A record must be kept for phase accumulation throughout the pulse sequence such that the associated coherence pathways of the different portions of recalled magnetisation do not result in additional echoes which might otherwise interfere with 
the stimulated echoes. By carefully managing the timing of the pulse sequence and strengths of the homo-spoil gradients used throughout the pulse sequence, the refocusing of previously recalled magnetisation can be avoided.

\subsection{Conclusions}

Using a pulsed magnetic field whose strength varies linearly in space, both spatial and displacement encoding can be achieved with NMR techniques. This chapter has provided the reader with the basic methods and theory of spatial and displacement encoding relevant to this research. When acquiring displacement space data, or $q$-space data, PGSE experiments using pulsed linear magnetic fields require a series of experiments to obtain all relevant displacement information. In the following chapter, the methods discussed in this chapter are combined, and with the use of a second order magnetic field, a technique for the parallel acquisition of all relevant displacement space data in a single scan experiment is proposed. 


\section{Chapter 5}

\section{Pulsed Second Order Field NMR}

\subsection{Introduction}

This chapter marks the beginning of the original work presented in this thesis. The second order magnetic field is described, and along with the spatial and displacement encoding techniques discussed in chapter 4 , all of these components are combined to develop the newly proposed methods of parallel data acquisition for real-time NMR measurements of homogeneous samples.

\subsection{Non-Linear Magnetic Fields}

\subsubsection{Effects and Uses of Non-Linear Magnetic Fields in NMR}

Traditionally, the magnetic field gradients used for spatial encoding in NMR possess a field strength which varies linearly in space. Although these fields are commonly referred to as linear gradients, they are in fact constant gradients since their gradient strength is constant in space. These fields are widely used for spatial encoding because the resulting linear relationship between spin precession frequency and position provides a straight forward mapping between frequency or phase and real space. Since electromagnetic gradient coils are never perfect in design, the gradient strength produced by these coils will 
have variations in space. In addition to this, other field inhomogeneities arise from unmatched magnetic susceptibilities in the sample and an imperfect $\mathbf{B}_{0}$ field. These field inhomogeneities can result in distortion of images and error in measurements.

In MRI, inhomogeneities in the $\mathbf{B}_{0}$ field and constant gradients result in a relationship between frequency or phase and position which is no longer linear, and may cause image distortions when a Fourier transform is applied directly to the $k$-space data to obtain an image. Some methods of image reconstruction and distortion correction are performed by remapping the image through knowledge of the magnetic fields. Once the magnetic fields have been mapped, a spherical harmonic decomposition of these field can be used to obtain the correct spatial orientations and sizes of the voxels comprising the image $[92,93]$. Other methods use correction functions which derive their parameters from imaging phantoms of well known dimensions, and apply these corrections to account for voxel shifts and variations in intensity due to non-linearities in the gradient fields [94, 95]. In NMR diffusion measurements, non-uniform gradients may cause signal attenuation to be misinterpreted due to a varying gradient strength across the sample, and has shown to induce errors of -10 to $20 \%$, and in extreme cases of non-uniformity, up to $30 \%$ overestimation of the diffusion coefficient [96]. Since magnetic susceptibility mismatches and non-linear gradient fields produced by gradient coils may result in a field not expected by a simple superposition of an ideal gradient field on the $\mathbf{B}_{0}$ field, care must be taken to analyse the result of such an arrangement. Work has been done to predict the effects of such situations in periodic porous media [19], general cases of non-uniform field gradients [97], and controlled variation of spatially distributed gradients [98]. Although many efforts are made to build gradient coils which produce a gradient which is highly constant in space, advantages arise from using fields over which the gradient value is spatially dependent.

Particular interest has been shown in the effects of so called parabolic fields which produce a gradient value which changes in a controlled manner with respect to position. Pure parabolic magnetic fields are impossible to produce due to Maxwell's equations necessitating the existence of concomitant fields. The fields referred to as parabolic are second order magnetic fields which vary in a quadratic fashion with respect to multiple axes. An imaging technique known as o-space imaging has been shown to provide an 
efficient parallel imaging method for multiple surface RF coils where the image is projected onto concentric rings using a second order field [99, 100]. The effect of second order fields on diffusion has also been investigated in detail for pulsed second order field coils $[84,101,102]$, as well as for the effects due to field profiles which may vary parabolically across pore spaces in which diffusion occurs [103].

\subsubsection{Second Order Magnetic Fields}

One of the second order magnetic fields used in this research is a field which produces a parabolic field profile along both the $x$ and $y$ axes. The first description provided in this subsection considers the case where the static magnetic field $\mathbf{B}_{0}$ lies along the $y$-axis. This is a highly unusual scenario in NMR, but since the majority of experiments performed in this research are performed on a magnet producing such a field, commonly referred to as Halbach geometry, this is the first case which is considered. To build a gradient coil which produces a parabolic field where the $y$-component of the magnetic field varies with respect to the $y$-axis, the desired relationship is

$$
B_{y}(y)=\frac{C}{2} y^{2}
$$

where $C$ is the curvature of the field determining the rate of change of the gradient value with respect to the position along the $y$-axis. From Maxwell's equations, $B_{y}$ must satisfy Laplace's equation (Eq. 6.4) such that

$$
\nabla^{2} B_{y}=\partial^{2} B_{y} / \partial x^{2}+\partial^{2} B_{y} / \partial y^{2}+\partial^{2} B_{y} / \partial z^{2}=0
$$

Substituting Eq. 5.1 into Eq. 5.2, it is found that Eq. 5.1 is not a solution, and there must exist a dependence of $B_{y}$ on $x, z$, or both. To have spatial dependence on only two axes and to satisfy Eq. 5.2, $\partial^{2} B_{y} / \partial x^{2}=-C$. Therefore, to obtain a parabolic dependence of $B_{y}$ along the $y$-axis, for this example, there must exist an equal and opposite parabolic dependence of $B_{y}$ along the $x$-axis such that

$$
B_{y}=\frac{C}{2}\left(x^{2}-y^{2}\right)
$$


and no matter what restrictions are imposed, there will always exist a spatial dependence of $B_{y}$ on a minimum of two axes for a second order magnetic field. This field, which produces a saddle shaped field strength profile in the $x y$ plane, is the field which is most similar to a parabolic field which can be created for a $\mathbf{B}_{0}$ field in the $y$ direction. The gradient strength of this field is given by $g=C \sqrt{x^{2}+y^{2}}$. Therefore, this field produces a gradient strength which varies linearly in space, making it a true linear gradient.

For NMR magnet geometry where the static magnetic field lies along the magnet bore or $z$ axis, commonly referred to as superconducting geometry, a field of $B_{z}=\frac{C}{2}\left(x^{2}-z^{2}\right)$ is desired. This field can be obtained simply by rotating the coil producing a $B_{y}=\frac{C}{2}\left(x^{2}-y^{2}\right)$ field $90^{\circ}$ about the $x$ axis. This field is also a linear gradient, and its gradient strength can be expressed as $g=C \sqrt{x^{2}+z^{2}}$.

\subsection{Second Order Field PGSE for Diffusion Measure- ments}

\subsubsection{The Basic Pulsed Second Order Field Sequence for Par- allel Acquisition of $q$-Space}

The parallel acquisition of $q$-space using pulsed second order magnetic fields in a PGSE pulse sequence is the basis of this research. The proposed pulse sequence is shown in Fig. 5.1. This modified PGSE pulse sequence combines the slice selection, read image, and uses a pulsed second order magnetic field to encode for displacement instead of a first order magnetic field.

The second order magnetic fields used in this research for Halbach geometry with $\mathbf{B}_{0}$ lying along the $y$ direction obey the relationship

$$
B_{y}=\frac{C}{2}\left(x^{2}-y^{2}\right)
$$




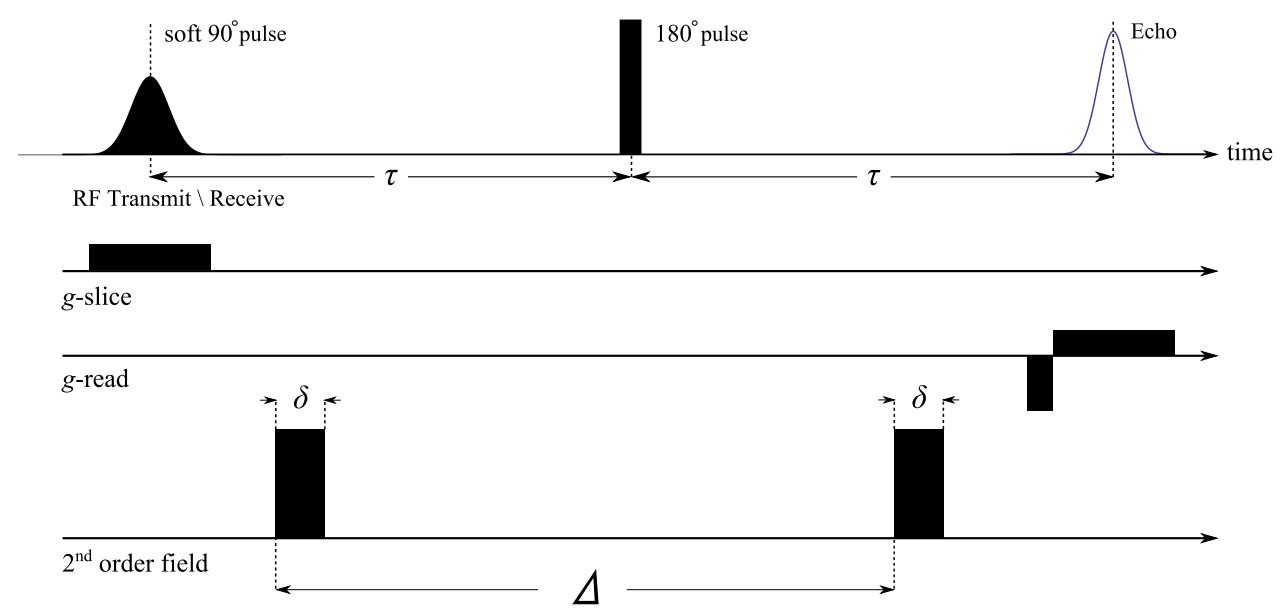

Figure 5.1: Proposed pulse sequence for the parallel acquisition of $q$-space.

and for a $\mathbf{B}_{0}$ field lying along the $z$ direction for superconducting geometry, they obey the relationship

$$
B_{z}=\frac{C}{2}\left(x^{2}-z^{2}\right),
$$

where $C$ is the curvature of the second order field. In this analysis the case for Halbach NMR magnet geometry is considered such that $\mathbf{B}_{0}$ lies along the $y$ axis. When only a thin slice of the sample is excited with a selective RF pulse and slice gradient, the dependence of the gradient strength due to the second order field on the slice direction can be neglected. For a thin slice taken in the $x$ direction about $x=0$, as $x \approx 0$ over the entire slice, the field strength experienced by the slice due to the second order magnetic field becomes $B_{y}=\frac{C}{2} y^{2}$, and appears parabolic along the $y$-direction (Fig. 5.2). The gradient of this field for the slice selected region in space will then vary linearly with respect to $y$ as $g=-C y$.

To discriminate between regions in space experiencing different gradient strengths, a read gradient is used to acquire a one dimensional profile along the length of the slice. From prior discussion in subsection 4.3.1 on the read image, it is recalled that the spin density at each point in space may be obtained by taking the Fourier transform of the echo during the application of a read gradient. Considering a homogeneous sample, this image would appear constant in amplitude over the sample when no second order field pulses 

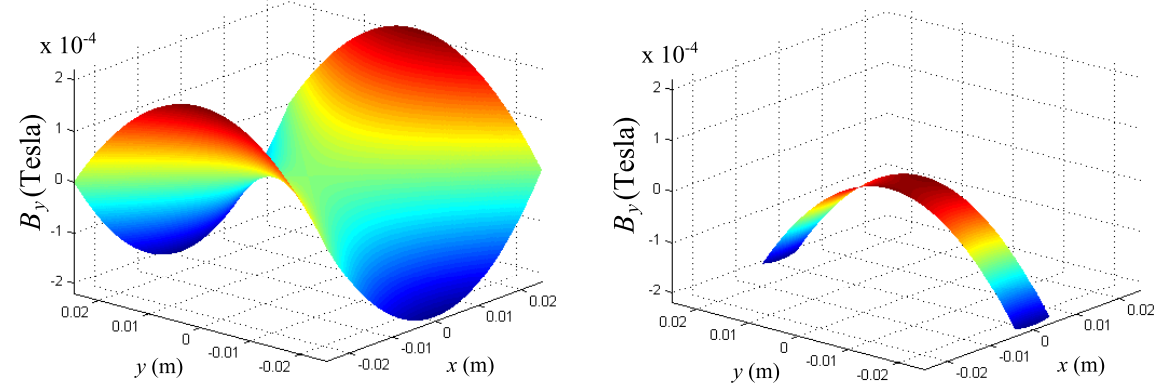

Figure 5.2: Magnetic field produced by the second order magnetic field coil. Left: Field profile of the $B_{y}$ component of the field over a large volume. Right: Field profile of the $B_{y}$ component of the field as seen by an $8 \mathrm{~mm}$ slice selected in the $x$-direction.

are applied. If the image is acquired with the inclusion of the second order field pulses, the position can be mapped onto the gradient value, and therefore, onto $q$ as shown in Fig. 5.3 using the relationship

$$
q(y)=-\gamma \delta C y
$$

The data set $E(q)$ is now acquired in parallel by taking the Fourier transform of a single
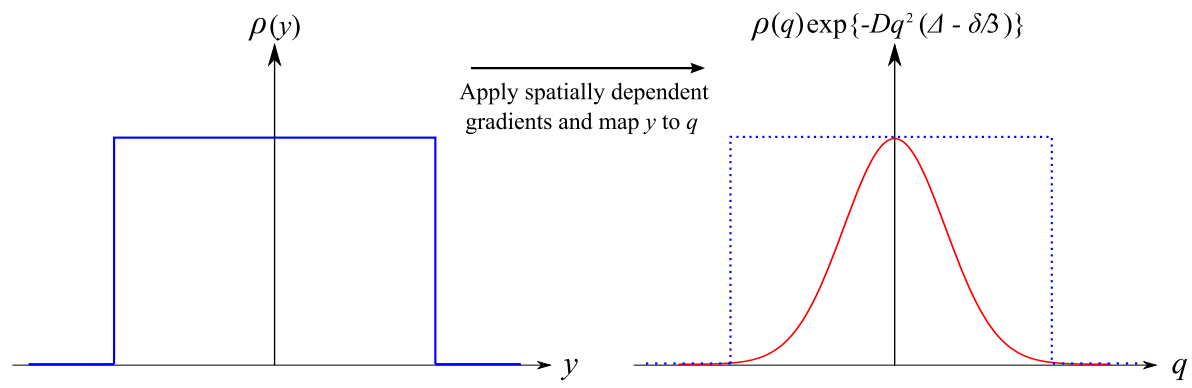

Figure 5.3: Left: Image acquired without the second order field pulses. Right: A diffusion encoded (solid red) image formed when the second order field pulses are included.

echo and mapping real space onto $q$-space through Eq. 5.6. With the data set $E(q)$ acquired in a single experiment, this allows the measurement of the diffusion coefficient and the average propagator in a single experiment. 


\subsubsection{Independence of Diffusion Measurements on Slice Thick- ness}

In the absence of a slice selection for the pulse sequence shown in Fig. 5.1, the real space to $q$-space mapping is lost, but the ability to measure the diffusion coefficient retained with an increase in signal to noise.

Considering the case for Halbach geometry, since the gradient strength of the second order field is now

$$
g=C \sqrt{x^{2}+y^{2}}
$$

for a given position $y$, a range of gradient strengths are sampled in the $x$ direction. To illustrate the relationship between the read image amplitude and the diffusion coefficient, an equation for the read image is developed in the following.

To derive the signal amplitude as a function of position along the $y$-read direction for an $x$-slice centred about $x=0$, the integral over the slice thickness must be taken for each position along the $y$-read direction. Integrating over the slice thickness $d$, and using Eq. 4.39 multiplied by the spin density $\rho$ for the signal contribution at each point in space, the signal as a function of $y$ becomes

$$
E(y)=\int_{-d / 2}^{d / 2} \rho(\mathbf{r}) \exp \left\{-q^{2} D \Delta\right\} d x .
$$

Here, the case of $\delta \ll \Delta$ has been considered such that $\Delta-\delta / 3$ from Eq. 4.39 has been replaced by $\Delta$ in Eq. 5.8. With $q=\gamma \delta g$, and the gradient magnitude $g=C \sqrt{x^{2}+y^{2}}$, Eq. 5.8 is rewritten as

$$
E(y)=\int_{-d / 2}^{d / 2} \rho(\mathbf{r}) \exp \left\{-\gamma^{2} \delta^{2} C^{2}\left(x^{2}+y^{2}\right) D \Delta\right\} d x
$$

The $y$-dependence of the exponent can now be removed from the integral. Defining an image modulation function $A(\rho, d)$ as

$$
A(\rho, d)=\int_{-d / 2}^{d / 2} \rho(\mathbf{r}) \exp \left\{-(\gamma \delta C x)^{2} D \Delta\right\} d x
$$


Eq. 5.9 may be written as

$$
E(y)=A(\rho, d) \exp \left\{-(\gamma \delta C y)^{2} D \Delta\right\}
$$

This is the result for the thin slice diffusion encoded image, modulated by the function $A(\rho, d)$. Here $\rho=\rho(\mathbf{r})$, and will contain information about the sample geometry, while $d$ contains information about the slice thickness. The integration limits in Eq. 5.10 may also be offset to account for a slice not centred about the origin. As a result, the modulation function may possess spatial dependence.

The function $A(\rho, d)$ can be evaluated for different sample geometries, slice thicknesses, and slice positions. For a square prism sample with faces perpendicular and parallel to the read gradient direction, this function will integrate to a constant with no dependence on $y$ for any slice thickness $d$. The diffusion encoded image will retain its Gaussian shape with only its amplitude affected by this factor. Since it is the variance of the Gaussian and not its amplitude which contains the diffusive information, a Gaussian curve may be fit directly to this image to determine $D$.

The function $A(\rho, d)$ can possess dependence on the read direction $y$ for other geometries. If $\int_{-d / 2}^{d / 2} \rho(x) d x$ is not constant across the sample in the read direction, this will be the case. For a slice thickness encompassing an entire cylinder whose axis is aligned along the $z$ direction, the integration limits become $-\sqrt{a^{2}-y^{2}}$ and $\sqrt{a^{2}-y^{2}}$, where $a$ is the radius of the cylinder. Performing the integral, and considering a homogeneous medium with uniform spin density within the sample, $A$ will assume the form

$$
A(y)=\rho \sqrt{\frac{\pi}{b}} \operatorname{Erf}\left(\sqrt{b a^{2}-b y^{2}}\right)
$$

where $b=\delta^{2} \gamma^{2} C^{2} D \Delta$. The fitting function used to determine $D$ from the image is no longer Gaussian, but the product of a Gaussian and the modulation function $A(y)$. If the cylinder's axis was oriented along the $y$ direction, the function $A(\rho, d)$ would integrate to a constant independent of position along the read direction, and a Gaussian could be fit directly to the image to determine $D$.

Choosing sample geometry and orientation in the second order magnetic field allows for 
simplification of the curve fitting process to determine the diffusion coefficient in the absence of slice selection. In this research only samples and sample orientations are chosen such that $A$ remains constant along the read direction, and a Gaussian curve can be fit directly to the diffusion encoded image to make the diffusion measurement.

It must be noted that this technique has been previously implemented by Loening et al. [84] using a $Z^{2}$ shim coil for superconducting geometry with a slightly different pulse sequence. Unlike the name of the coil may imply, no $Z^{2}$ coil can produce a field whose strength is only dependent on the $z$-axis, but instead produces a field of $B_{z}=C\left(z^{2}-y^{2} / 2-x^{2} / 2\right)$, a consequence of Maxwell's equations which are discussed in detail in subsection 6.2.2. Such a field makes it difficult to acquire $q$-space data in parallel as a well defined mapping between real space in one dimension and $q$-space is difficult to obtain, but can still be used to make single-shot diffusion measurements. Shim coils are not designed to be pulsed with high current or produce strong fields, and consequently are not well suited for the experiments proposed in this chapter. In the case of Loening et al. [84], long gradient pulses which began before spin excitation and ended during the storage period of a PGSTE pulse sequence were used such that the effects of eddy currents resulting from switching their shim coil on and off were minimised. Also, a long, weak read gradient was used to obtain a spectrum, and diffusion coefficients for multiple species were determined. This was not the case for the proposed experiments in this chapter as strong, short second order field pulses are used in conjunction with a true imaging gradient of significant strength to measure the diffusion coefficient of a single species.

\subsubsection{Validity of Diffusion Measurements Using $2^{\text {nd }}$ Order Fields}

So far it has been assumed that Eq. 4.39 holds for diffusive attenuation in the second order magnetic field for the proposed pulse sequence, and therefore the normalised signal as a function of the read direction $E_{N}(y)$ for an infinitely thin slice may be written as

$$
E_{N}(y)=\exp \left\{-(\gamma \delta C y)^{2} D \Delta\right\}
$$


where $g=\gamma \delta C y$. This result is based on the assumption that a diffusing spin has experienced dephasing and rephasing gradient pulses which are matched in strength. Since displacements are being measured in a gradient field whose strength varies is space, this is not the case, and the validity of this assumption must be assessed.

To verify Eq. 5.13, the signal amplitude as a function of position is derived starting with the phase and amplitude contribution to each point in space by spins diffusing within the sample. The phase $\phi$ accumulated by a spin diffusing in the second order magnetic field due to the dephasing and rephasing diffusion gradient pulses is

$$
\phi=\delta \gamma\left[\frac{C}{2}\left((y-Y)^{2}-y^{2}\right)\right],
$$

where $Y$ is the displacement occurring during the observation time $\Delta$. The amplitude contribution to the signal for diffusion in the Gaussian regime is given by the free diffusion propagator

$$
\bar{P}_{D}(Y, \Delta) \propto \exp \left\{\frac{Y^{2}}{4 D \Delta}\right\}
$$

a normal probability distribution for displacement $Y$.

For this analysis a few assumptions must be made. It is first assumed that diffusion during the application of the gradient pulses can be neglected for $\delta \ll \Delta$. It is then assumed that the read image is acquired instantaneously after the rephasing diffusion gradient pulse, a reasonable assumption for a short acquisition window. With these assumptions, the signal as a function of position is obtained by integrating the amplitude and phase contribution to a given point $y$ in the read image due to all spins such that

$$
E_{N}(y)=\frac{1}{\sqrt{4 D \Delta \pi}} \int \exp \left\{-\frac{Y^{2}}{4 D \Delta}\right\} \exp \left\{\mathrm{i} \gamma \delta \frac{C}{2}\left((y-Y)^{2}-y^{2}\right\} d Y\right. \text {. }
$$

Expanding the term in the phase contribution of the integral, and expressing the phase as a product of two exponentials, Eq. 5.16 can be rewritten as

$$
E_{N}(y)=\frac{1}{\sqrt{4 D \Delta \pi}} \int \exp \left\{-\frac{Y^{2}}{4 D \Delta}\right\} \exp \left\{\mathrm{i} \gamma \delta \frac{C}{2} Y^{2}\right\} \exp \{-\mathrm{i} \delta \gamma C y Y\} d Y
$$


When considering the normal distribution of the propagator, it is noted that only spins undergoing displacement within the diffusion length $d_{l}=\sqrt{2 D \Delta}$ will contribute significantly to the signal at position $y$. This means that only displacements of $|Y| \leq \sqrt{2 D \Delta}$ will be considered. A characteristic length scale $l_{c}$ of the second order field can then be defined as

$$
l_{c}=\sqrt{\frac{2}{\gamma \delta C}} .
$$

A condition for simplification of Eq. 5.17 can then be defined as

$$
l_{c} \gg|Y|
$$

If the condition in Eq. 5.19 holds for all relevant values of $Y$, in this case for all $|Y| \leq$ $\sqrt{2 D \Delta}$, then

$$
\exp \left\{\gamma \delta \frac{C}{2} Y^{2}\right\} \approx 1
$$

and Eq. 5.17 can be rewritten as

$$
E_{N}(y) \approx(4 D \Delta \pi)^{-1 / 2} \int \exp \left\{-\frac{Y^{2}}{4 D \Delta}\right\} \exp \{-\mathrm{i} \gamma \delta C y Y\} d Y
$$

This integral can be evaluated, and the familiar form obtained

$$
E_{N}(y) \approx \exp \left\{-(\gamma \delta C y)^{2} D \Delta\right\}
$$

The condition in Eq. 5.19 relates to the linearity of the second order field over relevant displacements in the field. Depending on the degree of curvature $C$ and pulse duration $\delta$, linear approximation of the local second order field will be accurate over displacements $Y$ which do not exceed some maximum value. Therefore, if the second order field is well approximated by a linear field over a range which encompasses all relevant displacements, the result for diffusion in a second order field is well approximated by that for diffusion in local constant gradients.

Given that the second order field is well approximated by a local linear field over displace- 
ments $Y$ during the observation time $\Delta$, it should also be well approximated by a local linear field during a finite diffusion gradient pulse duration of $\delta$. With this assumption, corrections for the finite duration of the diffusion gradient pulses should hold, such as the familiar replacement of $\Delta$ by $\Delta-\delta / 3$ in Eq. 5.22 such that

$$
E_{N}(y) \approx \exp \left\{-(\gamma \delta C y)^{2} D(\Delta-\delta / 3)\right\}
$$

This is a result which forms the basis for the diffusion measurements presented in chapter 7 of this thesis.

\subsubsection{Multiple Observation Time Diffusion Measurements with a Second Order Field in Difftrain}

The implementation of the pulsed second order magnetic field and read gradient in the pulse sequence Difftrain, as described in section 4.4.6, is illustrated in Fig. 5.4. This is analogous to the implementation of the read gradient and second order fields in the PGSE pulse sequence for single-shot diffusion measurements. Since only a fraction of the stored magnetisation is recalled for each echo in Difftrain, no slice selection is employed such that the entire sample volume is excited, and the amount of stored magnetisation and signal to noise for each echo is maximised. Since Difftrain harbours undesirable coherence pathways, the phase history and evolution of spins must be carefully managed to prevent unwanted refocusing of magnetisation which may interfere with the desired displacement encoded images.

The modifications made to the conventional Difftrain pulse sequence begin with the inclusion of a read gradient. The read gradient is essential as it provides a 1D image along the length of the slice. This allows discrimination between regions experiencing different gradients strengths such that the image may be related to the diffusion coefficient. Similar to the stimulated echo and conventional Difftrain pulse sequences, a homo-spoil gradient pulse is applied between the storage and first recall RF pulses, and after each echo to spoil any magnetisation remaining in the transverse plane. Unlike the conventional DiffTrain pulse sequence, the second order magnetic field used to encode for diffusion will differ in 


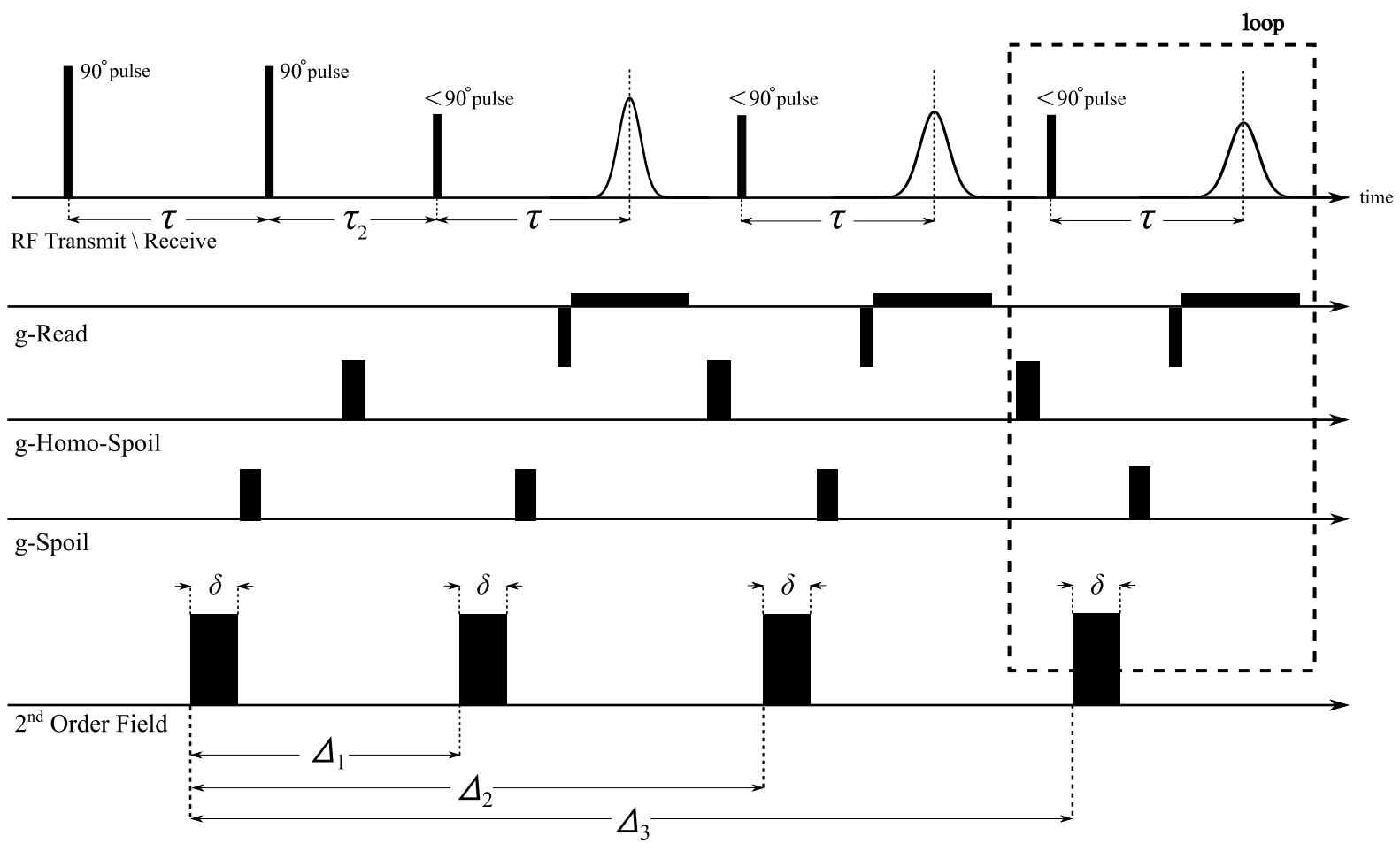

Figure 5.4: The modified DiffTrain pulse sequence including components enabling measurement of the apparent diffusion coefficient at multiple encoding times.

how it spoils the FID resulting from the recall RF pulses.

Each recall RF pulse will excite magnetisation without phase history into the transverse plane causing a free induction decay (FID). Such magnetisation is further referred to as unwanted magnetisation as it may interfere with the desired stimulated echo signals. Phase cycling is usually performed to cancel out these effects. Since a single-shot experiment is desired, no phase cycling can be implemented, and spoiler gradient pulses must be used to dephase this unwanted magnetisation. Normally, the gradient pulses used to encode for diffusion in Difftrain act as spoilers. With the diffusion gradient pulses applied by a second order field, the unwanted magnetisation is no longer spoiled in the same fashion because the second order field does not have a constant gradient strength in space, and close to the origin the gradient strength is weak. This results in unwanted magnetisation insufficiently spoiled about the origin. For this reason, an additional constant 
gradient field pulse is used as a spoiler after each recall RF pulse to uniformly dephase the unwanted magnetisation. In order to prevent spoiling of the stimulated echo, a pulse of equal duration and amplitude is applied before the storage RF pulse. By doing this, the spoiler after the recall pulse refocuses the desired echo while spoiling the unwanted magnetisation. Since diffusion is occurring in the time interval between these spoiler pulses, there will not be complete refocusing of the echo, and some signal attenuation will be induced by these pulses. For a conventional diffusion measurement this effect would need to be carefully accounted for. In this case, the diffusion measurement is made by fitting a curve to the read image, with the curve shape, and not its amplitude, providing the diffusion measurement. With signal attenuation due to these spoiler pulses constant across the entire image, these effects are of no consequence other than a decreased image amplitude, and do not affect the diffusion measurement.

The result of this scheme is a series of stimulated echoes, each encoded with displacement information for a different observation time. With each echo producing a diffusion encoded image, and each image providing a measure of the diffusion coefficient for the corresponding observation time, the diffusion coefficient is measured at multiple observation times in a single experiment.

\subsubsection{Incorporation of a 13-Interval Pulse Sequence in the Mod- ified Difftrain Method}

When using a stronger $B_{0}$ field, significant internal gradients can arise from susceptibility mismatching inside the pore space of porous media. Since the internal gradients act to dephase the spin ensemble, this will cause a loss of net magnetisation, and a reduced signal to noise in the desired echo signals. To minimise this effect, a 13-interval pulse sequence was incorporated into the modified Difftrain pulse sequence [22, 104].

The pulse sequence begins similarly to the before mentioned modified Difftrain pulse sequence in subsection 5.2.4, but breaks up the initial displacement encoding into two separate pulses. These pulses are of opposite amplitude, but because they are separated by a $180^{\circ} \mathrm{RF}$ pulse, they act to impart phase in the same direction. The $90^{\circ}$ storage $\mathrm{RF}$ pulse is applied a time $2 \tau$. If no gradient pulses had been applied, this would be the 


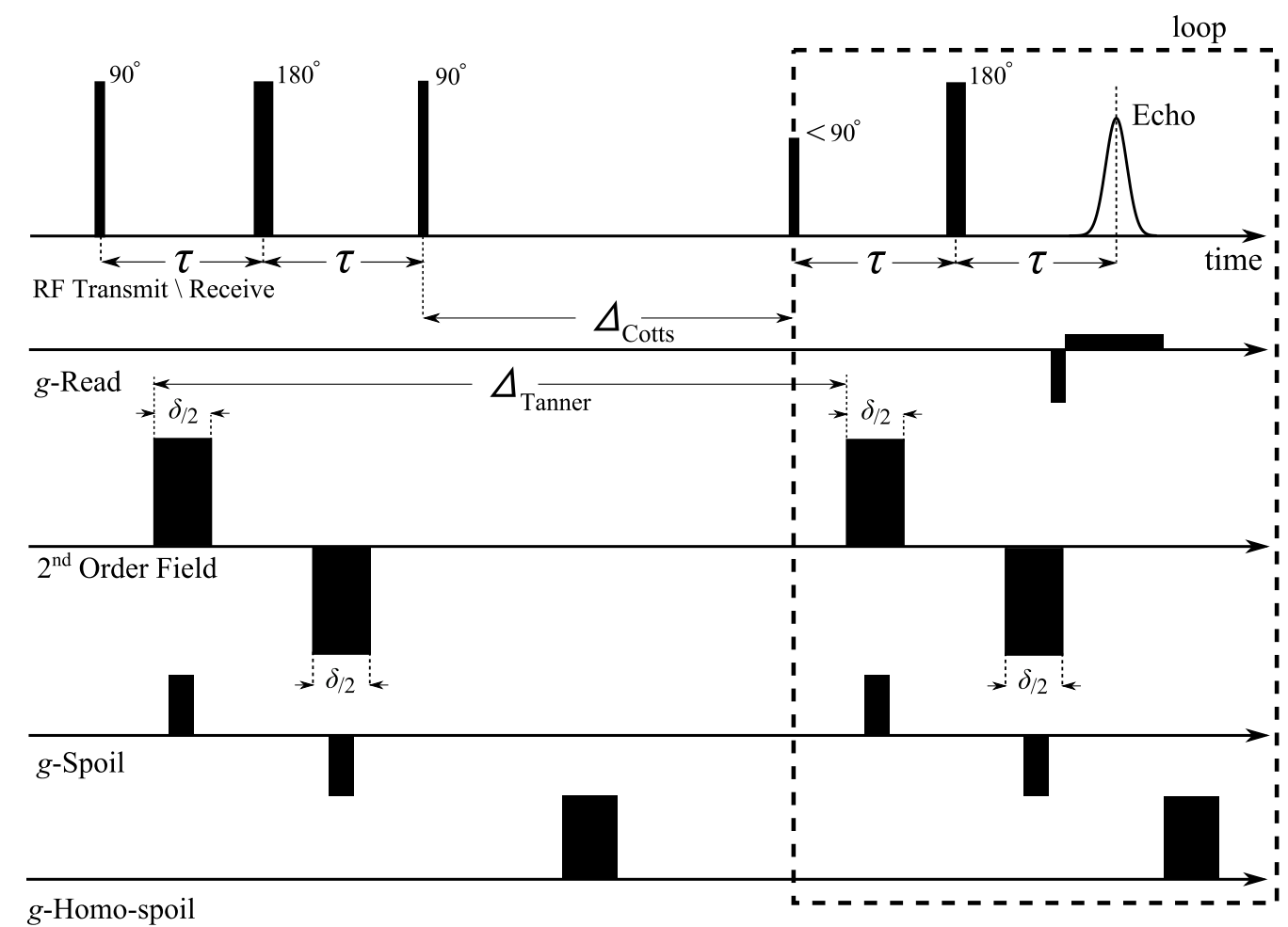

Figure 5.5: Modified Difftrain pulse sequence for a single-shot measurement of $D_{a p p}(\Delta)$ at multiple $\Delta$ with incorporation of a 13 -interval pulse sequence.

point in time where a Hahn echo would form. Therefore, the storage RF pulse stores the prepared magnetisation at a point in time where the effects of the internal gradients are minimised.

The effective observation time for each echo will increase as the looped section of the pulse sequence is repeated. Tanner [105] defined $\Delta$ as the time between the beginning of the dephasing and rephasing diffusion gradient pulses, and Cotts et al. [104] defined $\Delta$ as the storage time. Using the notation in Fig. 5.5, the $i^{\text {th }}$ echo will have an effective corresponding observation time of

$$
\Delta_{i, \mathrm{eff}}=\Delta_{\mathrm{Cotts}}+(3 \tau / 2)-\delta / 12+(i-1) t_{\mathrm{loop}},
$$


where $t_{\text {loop }}$ is the time it takes to perform the looped portion of the pulse sequence $[1,104]$. To help avoid any unwanted refocusing of magnetisation from previous recall RF pulses, the homo-spoil gradient was varied from loop to loop [22].

This version of the modified Difftrain pulse sequence, unlike the version shown in Fig. 5.4 and described in subsection 5.2.4, will correct for background gradients due to field inhomogeneities and internal gradients caused by susceptibility mismatches in the sample. Although this version is complex and more difficult to implement, some porous media cannot be characterised in a single-shot technique without the incorporation of the 13interval pulse sequence as internal gradients would otherwise decrease the signal to noise to an insufficient level.

\subsection{Time domain signal as the average propagator}

\subsubsection{Introduction}

As discussed in subsection 5.2.1, the pulse sequence for the parallel acquisition of $q$ space can be used to acquire the data set $E(\mathbf{q})$ in a single scan by mapping the real space image to $q$-space. With the inverse Fourier transform of the real space image yielding the time domain signal (i.e. the echo), and the inverse Fourier transform of $E(\mathbf{q})$ yielding the average propagator, since the real space image is $E(\mathbf{q})$ under a linear mapping between space and $\mathbf{q}$, it follows that the echo itself is the average propagator under a linear mapping between time and displacement. Therefore, the pulse sequence described in subsection 5.2.1 providing the parallel acquisition of $q$-space is the method through which the time domain signal directly yields the average propagator. In this section, the theory behind this phenomenon will be discussed in detail.

\subsubsection{Comparison to other displacement measurements}

Temporal resolution of transient or short lived processes requires cutting edge NMR techniques, which result in a loss of spatial resolution or signal to noise for increased temporal resolution. Advances in hardware, along with post processing techniques such 
as Bayesian approaches and compressed sensing, have resulted in rapid imaging techniques [43-46], the fastest of which are able to resolve transient processes on the order of tens of milliseconds $[35,106,107]$. Although they are extremely fast, for a high resolution a large number of pixels are necessary which reduces the signal to noise per pixel and increases experimental time. In addition to this, they are only able to provide a mean velocity per pixel, and can suffer from artefacts arising from motion in the presence of the imaging gradients. The pulsed gradient spin-echo NMR technique can provide a full probability distribution for displacements known as the average propagator [13, 41, 50], but requires a series of experiments over an extended time, and cannot resolve transient and quickly changing processes. When the total experimental time for a series of PGSE experiments is reduced, the signal to noise, and or displacement resolution, is also reduced. The NMR technique presented in this thesis allows for the preservation of displacement resolution in the average propagator with a modified PGSE experiment, while achieving temporal resolution on the order of the fastest velocity imaging techniques available.

\subsubsection{Single-shot propagator measurement}

The single-shot propagator measurement shown in Fig. 5.6 is similar to the sequence shown in Fig. 5.1, but uses timing which is better defined to simplify the analysis presented in the following discussion. The single-shot flow propagator measurements presented in the experimental results in chapter 7 of this thesis were performed in a superconducting magnet where $B_{0}$ lies along the $z$ direction. Therefore, the second order field used for those experiments obeys the relation $B_{z}=C\left(x^{2}-z^{2}\right) / 2$, where $C$ is the curvature of the field. With a thin slice selection taken in the $x$ direction and centred about $x=0$, the field experienced by the thin slice due to the second order field can be approximated as $B_{z} \approx C z^{2} / 2$, and appears parabolic. Since the displacement encoding is performed along the $z$-axis, a $z$-read gradient will be applied during the acquisition window to form the read image.

To determine the read image amplitude as a function of $z$, the signal can be represented by the integral of the average propagator $\bar{P}(Z, \Delta)$ for all spins which have undergone some displacement $Z$ during the observation time $\Delta$, which are at position $z$ during the 


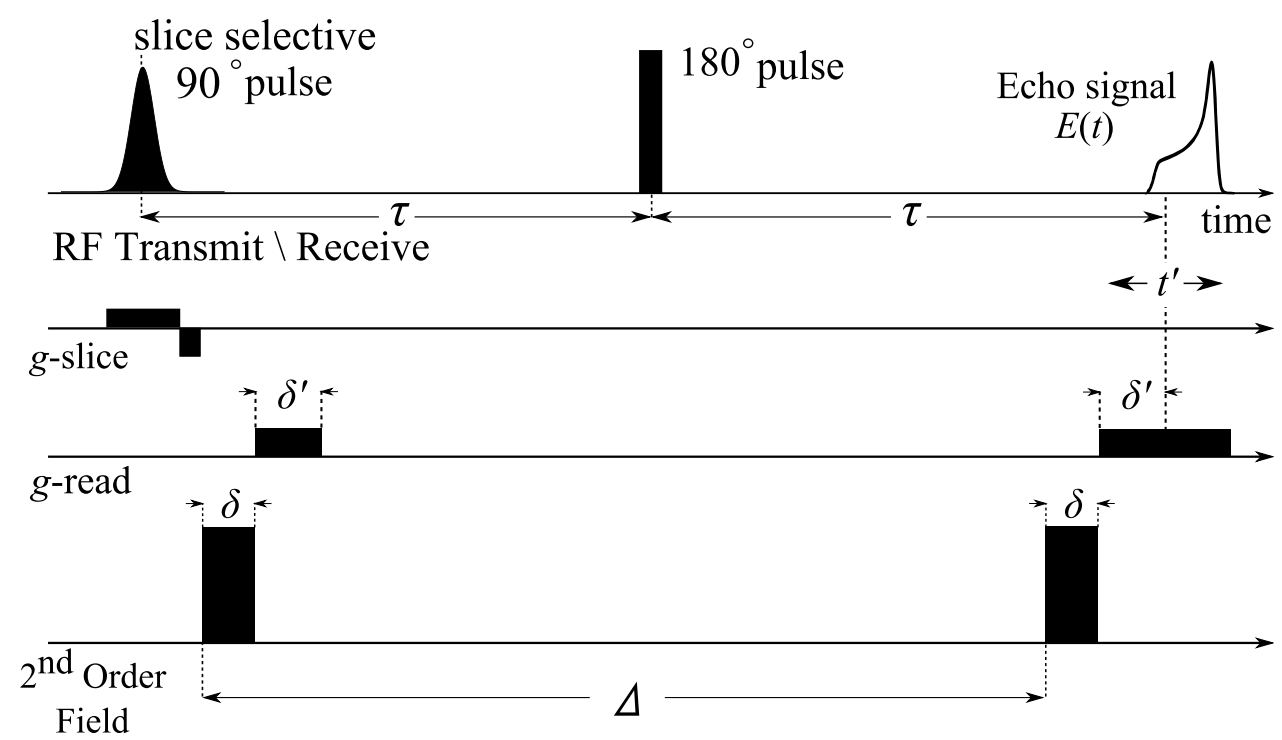

Figure 5.6: Single-shot PGSE-imaging sequence for propagator measurement. The lines $g$-slice and $g$-read depict the pulsed linear magnetic fields used for slice selection in the $x$ direction and the imaging read gradient in the $z$ direction respectively. The second order field is used to encode for displacement. A Gaussian shaped $90^{\circ}$ pulse is used in conjuction with the $g$-slice gradient to excite a thin slice volume of Guassian profile along the $x$ direction.

acquisition window, multiplied by the corresponding phase factor $\exp \left\{\mathrm{i} \phi_{2^{\text {nd }}}(z, Z)\right\}$ such that

$$
E(z) \propto \int \bar{P}(Z, \Delta) \exp \left\{\mathrm{i} \phi_{2^{\text {nd }}}(z, Z)\right\} d Z .
$$

The phase imparted to spins undergoing displacement $Z$ during $\Delta$ from the first second order field pulse will be

$$
\phi_{2^{n d}, 1}=\gamma \delta C(z-Z)^{2} / 2
$$

The phase for these same spins due to the second second order field pulse will be

$$
\phi_{2^{n d}, 2}=\gamma \delta C z^{2} / 2
$$


The phase shift due to the second order field pulses can then be expressed as the difference of Eq. 5.26 and Eq. 5.27 such that

$$
\phi_{2^{\text {nd }}}(z, Z)=\gamma \delta \frac{C}{2}\left[(z-Z)^{2}-z^{2}\right]
$$

Substituting for $\phi_{2^{n d}}$ in Eq. 5.25, and representing the phase term as the product of two exponentials, Eq. 5.25 can be rewritten as

$$
E(z)=\int P(Z, \Delta) \exp \{-\mathrm{i} \gamma \delta C z Z\} \exp \left\{\mathrm{i} \gamma \delta \frac{C}{2} Z^{2}\right\} d Z
$$

Using the length scale defined in Eq. 5.18 where

$$
l_{c}=\sqrt{\frac{2}{\gamma \delta C}},
$$

if $l_{c} \gg|Z|^{\max }$, where $|Z|^{\max }$ is the maximum possible displacement, then the term $\gamma \delta \frac{C}{2} Z^{2} \ll 1$, and $\exp \left\{\mathrm{i} \gamma \delta \frac{C}{2} Z^{2}\right\} \approx 1$. With the gradient strength due to the second order field expressed as $g \approx-C z$, it follows that

$$
q=-\gamma \delta C z
$$

and Eq. 5.29 can be rewritten as

$$
E(z)=\int P(Z, \Delta) \exp \{\mathrm{i} q Z\} d Z
$$

Eq. 5.32 now represents a Fourier relationship between the encoded read image and the average propagator. Since the inverse Fourier transform of the encoded read image under the real space to $q$-space mapping (Eq. 5.31) is the average propagator, and the inverse Fourier transform of the real space image is the echo, it follows that the echo, when normalised and plotted against displacement space, is the average propagator.

The mapping between time and displacement space can be obtained by first calculating 
the field of displacements $(F O D)$ as

$$
F O D=\frac{1}{\Delta q}
$$

where $\Delta q$ is the spacing of data points in $q$ after mapping real space onto $q$-space with Eq. 5.31. By replacing the range of time in the acquisition window with the FOD, time is mapped onto displacement space.

\subsubsection{Long displacement and strong curvature limit}

For an entirely real echo representing the average propagator, the condition $l_{c} \gg|Z|^{\max }$, and therefore $\exp \left\{\mathrm{i} \gamma \delta \frac{C}{2} Z^{2}\right\} \approx 1$ must hold. If there is strong curvature and duration of the second order field $\delta C$, and displacements $Z$ are large, this condition will not hold. However, the average propagator can still be recovered by applying a phase correction to the echo.

From Eq. 5.29, a substitution can made by defining an average propagator which contains phase information $\bar{P}_{\phi}(Z, \Delta)$, such that

$$
\bar{P}_{\phi}(Z, \Delta)=\bar{P}(Z, \Delta) \exp \left\{\mathrm{i} \gamma \delta \frac{C}{2} Z^{2}\right\}
$$

Eq. 5.29 can then be rewritten as

$$
E(z)=\int \bar{P}_{\phi}(Z, \Delta) \exp \{-\mathrm{i} \gamma \delta C z Z\} d Z
$$

Substituting $q=-\gamma \delta C z$, Eq. 5.35 is rewritten as

$$
E(z)=\int \bar{P}_{\phi}(Z, \Delta) \exp \{-\mathrm{i} q Z\} d Z
$$

The $z$ axis may again be rescaled with the relation from Eq. 5.31, and real space mapped onto $q$-space. This time, a Fourier relationship is revealed between $q$-space and complex displacement space. Since the inverse Fourier transform which is used to yield $\bar{P}_{\phi}$ is the acquired echo, which can be complex, this implies that the mapping between time and 
displacement will now be complex. To derive the complex mapping between time and displacement, the formation of the echo from spins being displaced between second order field pulses is studied in more detail.

\subsubsection{New echo condition}

The relationship between time and displacement space is most easily obtained starting with the expression for the echo condition $\int \mathbf{g}^{*}(t) d t=0$. Using pulsed second order fields, the gradient strength becomes dependent on position, and the new echo condition can now be represented as

$$
\int g^{*}(z(t), t) d t=0
$$

Assuming a narrow pulse approximation such that no displacement occurs during the second order field pulses, and letting time $t^{\prime}$ represent time measured from the centre of the acquisition window in the positive and negative direction as illustrated in Fig. 5.6, Eq. 5.37 can be rewritten as

$$
-C \delta Z-g_{R} t^{\prime}=0
$$

for particles experiencing displacement $Z$ during the observation $\Delta$, where $g_{R}$ is the strength of the read gradient. Rearranging Eq. 5.38 for $Z$, the relationship between time and displacement space is obtained

$$
Z=-\frac{g_{R} t^{\prime}}{C \delta}
$$

This time dependent echo condition, and mapping between time and displacement space, provide insight into how the echo represents the average propagator. The echo signal measured in the time domain is no longer the simultaneous refocusing of the entire spin ensemble as in conventional PGSE, but is now a continuous series of individual echoes (Fig. 5.7). Each of these echoes is formed by the refocusing of a subset of spins having experienced the same displacement $Z$ during the observation time, with an amplitude directly related to the number of spins in the subset. Taking the magnitude of the echo, normalising it such that its integral is unity, and mapping it to displacement space 
using Eq. 5.39, it becomes the probability distribution for displacement, and the average propagator measured directly through acquisition of the entire echo signal (Fig. 5.7). This

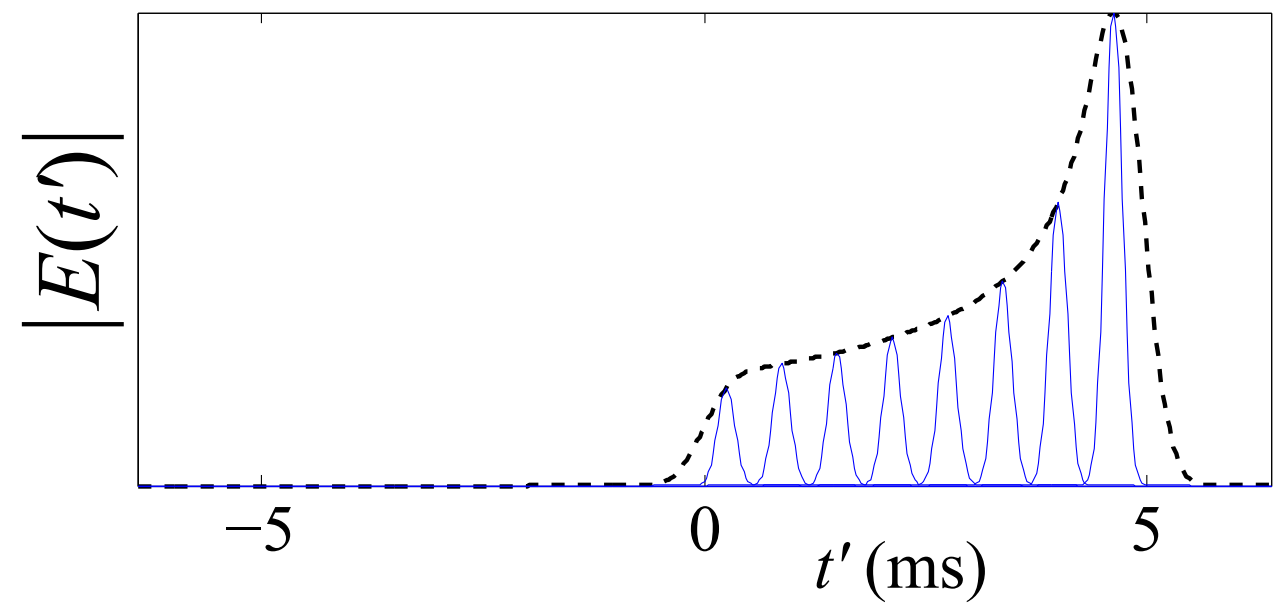

Figure 5.7: The amplitude of an echo formed from the pulse sequence shown in Fig. 5.6 for an undefined flow is plotted against $t^{\prime}$, the time measured from the centre of the acquisition window. The entire echo signal is now composed of a continuous series of individual echoes. Each individual echo is formed by the refocusing of a subset of spins which has experienced the same displacement $Z$ during the observation time $\Delta$, and has an amplitude proportional to the number of spins in the subset. Under the mapping in Eq. 5.39 and normalisation of the entire echo signal $E\left(t^{\prime}\right)$, this represents the average propagator $\bar{P}(Z, \Delta)$.

result, and the full complex mapping between time and displacement space, can also be obtained without a narrow pulse approximation by accounting for phase accumulation throughout the pulse sequence.

\subsubsection{Phase Evolution Echo Derivation}

In the previous subsection 5.3.5, the mapping between time and displacement was obtained, but no phase information was available. In this and the following subsection (5.3.6 \& 5.3.7), the mapping between time and displacement, and the phase correction for the echo to obtain the average propagator, and respectively derived in detail by carefully analysing the phase evolution of the system. 
The complete evolution of phase $\phi$ for a spin throughout the proposed pulse sequence in Fig. 5.6 can be determined by integrating the angular frequency off resonance

$$
\omega_{o s}(t)=\gamma B_{z}^{*}(z(t))
$$

such that

$$
\phi=\gamma \int_{0}^{t} B^{*}(z(t)) d t
$$

where $z(t)$ represents the path traversed in the $z$ direction, and $B^{*}(z(t))$ is the effective magnetic field applied by the pulsed gradients as a spin moves along some path. The function $B^{*}$ is similar to the effective gradient $g^{*}$ in the sense that it changes sign upon application of a $180^{\circ} \mathrm{RF}$ pulse. In this analysis, Eq. 5.41 will be evaluated for the subset of spins starting at position $z_{0}$ at the beginning of the first second order field pulse, which undergo displacement $Z$ during the observation time $\Delta$. This integral can be evaluated separately for each gradient pulse. For the first second order field pulse, the position dependent magnetic field in time is taken as $\frac{\gamma C}{2}(z(t))^{2}$, where $z(t)=z_{0}+Z_{p}(t)$, with $Z_{p}(t)$ representing a time dependent displacement path. Evaluating Eq. 5.41 for the first second order field pulse yields

$$
\phi_{2^{\text {nd }, 1}}=\frac{\gamma C}{2} \int P\left(Z_{p}\right) \int_{0}^{\delta}\left[z_{0}+Z_{p}(t)\right]^{2} d t d Z_{p},
$$

where $P\left(Z_{p}\right)$ denotes the probability of particles traversing some path $Z_{p}$, and the integral over $d Z_{p}$ is taken over all possible paths. As it is only the subset of spins undergoing displacement $Z$ during the observation time $\Delta$ which are being considered, the position of the particles during the second second order field pulse will be $z(t)=z_{0}+Z+Z_{p^{\prime}}(t)$. The phase due to the second second order field pulse can then be expressed as

$$
\phi_{2^{\text {nd }, 2}}=\frac{-\gamma C}{2} \int P\left(Z_{p^{\prime}}\right) \int_{0}^{\delta}\left[z_{0}+Z+Z_{p^{\prime}}(t)\right]^{2} d t d Z_{p^{\prime}} .
$$


Since the terms $z_{0}$ and $Z$ have no path dependence, the integrals for these terms can be performed, and the phase accumulations for each of the second order field pulses rewritten as

$$
\phi_{2^{\text {nd }, 1}}=\frac{\gamma C}{2}\left\{z_{0}^{2} \delta+\int P\left(Z_{p}\right) \int_{0}^{\delta}\left[2 z_{0} Z_{p}(t)+Z_{p}^{2}(t)\right] d t, d Z_{p}\right\}
$$

and

$$
\phi_{2^{\text {nd }, 2}}=-\frac{\gamma C}{2}\left\{z_{0}^{2} \delta+2 z_{0} Z \delta+Z^{2} \delta+\int P\left(Z_{p^{\prime}}\right) \int_{0}^{\delta}\left[2 z_{0} Z_{p^{\prime}}(t)+2 Z Z_{p^{\prime}}(t)+Z_{p^{\prime}}^{2}(t)\right] d t d Z_{p^{\prime}}\right\} .
$$

Here it is noted that the probability distributions for all possible paths during the first and second second order field pulses are equal, and therefore

$$
\begin{aligned}
& \int P\left(Z_{p}\right) \int_{0}^{\delta}\left[2 z_{0} Z_{p}(t)+Z_{p}^{2}(t)\right] d t d Z_{p} \\
= & \int P\left(Z_{p^{\prime}}\right) \int_{0}^{\delta}\left[2 z_{0} Z_{p^{\prime}}(t)+Z_{p^{\prime}}^{2}(t)\right] d t d Z_{p^{\prime}} .
\end{aligned}
$$

Summing Eq. 5.44 and Eq. 5.45, and cancelling terms using the relationship from 5.46, the total phase due to the second order field pulses $\phi_{2^{\text {nd }}}$ is expressed as

$$
\phi_{2^{\text {nd }}}=\frac{-\gamma C}{2}\left[2 z_{0} Z \delta+Z^{2} \delta+\int P\left(Z_{p^{\prime}}\right) \int_{0}^{\delta} 2 Z Z_{p^{\prime}}(t) d t d Z_{p^{\prime}}\right]
$$

Up until the centre of the acquisition window, the read gradient pulses will result in signal attenuation due to diffusion, and a net phase shift $\phi_{r, 0}$ due to flow for any subset of spins undergoing the same displacement $Z$ during $\Delta$. For this reason, it is convenient to define the phase imparted by the read gradients as a function of $t^{\prime}$, the time measured from the centre of the acquisition window, which can be positive and negative. The position of a spin at time $t^{\prime}$ can then be represented as $z_{0}+Z+Z^{\prime}+Z_{p^{\prime \prime}}(t)$, where $Z^{\prime}$ is a displacement occurring between the beginning of the second second order pulse and the centre of the acquisition window, and $Z_{p^{\prime \prime}}$ is a time dependent displacement path. The 
total phase shift $\phi_{r}$ from the centre of the acquisition window due to the read gradient pulses can then be expressed as

$$
\phi_{r}=\phi_{r, 0}-\gamma g_{R} \int_{-\infty}^{\infty} P\left(Z^{\prime}, \delta+\delta^{\prime}\right) \int P\left(Z_{p^{\prime \prime}}\right) \int_{0}^{t^{\prime}}\left(z_{0}+Z+Z^{\prime}+Z_{p^{\prime \prime}}(t)\right) d t d Z_{p^{\prime \prime}} d Z^{\prime}
$$

where $P\left(Z^{\prime}, \delta+\delta^{\prime}\right)$ is the probability a spin has been displaced a distance $Z^{\prime}$ during the time $\delta+\delta^{\prime}$, and $\delta+\delta^{\prime}$ is the time between the beginning of the second second order pulse and the centre of the acquisition window. Here, the integrals can be performed for the $z_{0}$ and $Z$ terms as they are independent on the path and $Z^{\prime}$. Also, the $Z^{\prime}$ term has no path dependence, and the $Z_{p^{\prime \prime}}(t)$ term has no $Z^{\prime}$ dependence. Therefore, Eq. 5.48 can be rewritten as

$$
\phi_{r}=\phi_{r, 0}-\gamma g_{R} t^{\prime} z_{0}-\gamma g_{R} Z \int_{-\infty}^{\infty} Z^{\prime} P\left(Z^{\prime}, \delta+\delta^{\prime}\right) d Z^{\prime}-\gamma g_{R} \int P\left(Z_{p^{\prime \prime}}\right) \int_{0}^{t^{\prime}} Z_{p^{\prime \prime}}(t) d t d Z_{p^{\prime \prime}}
$$

The total phase $\phi_{t o t}$ due to all gradient pulses can be expressed as

$$
\phi_{t o t}=\phi_{r}+\phi_{2^{\text {nd }}}
$$

When $\phi_{t o t}$ is independent of $z_{0}$, all spins which have undergone displacement $Z$ during the observation time $\Delta$ will be in phase. Setting the sum of terms containing $z_{0}$ from $\phi_{t o t}$ equal to zero, and recalling that $\phi_{r, 0}$ is independent of $z_{0}$, this results in the relationship

$$
-\gamma C z_{0} Z \delta-\gamma g_{R} z_{0} t^{\prime}=0
$$

Rearranging Eq. 5.51 for $Z$ in terms of $t^{\prime}$,

$$
Z=-\frac{g_{R} t^{\prime}}{C \delta}
$$

and the previously derived mapping between time and displacement is once again obtained without a narrow pulse approximation. 


\subsubsection{Complex time to real displacement mapping through phase evolution}

To determine the exact complex relationship between time and displacement, the echo condition from Eq. 5.52 is substituted into $\phi_{t o t}$, and the bulk phase shift due to the read gradients evaluated. Complete knowledge of all probabilities presented in this derivation is required to make this calculation, which may not be available. In fact, if all information about the probabilities is known, there is no need to measure the average propagator. However, if the assumption is made that the spins possess a constant velocity throughout the experiment, Eq. 5.41 can be evaluated by expressing the time dependent position as

$$
z(t)=z_{0}+v t
$$

where $v$ is the velocity. Letting $v=Z / \Delta$, the time dependent position is rewritten as

$$
z(t)=z_{0}+\frac{Z t}{\Delta}
$$

Using the assumption in Eq. 5.54, evaluation of Eq. 5.41 for the second order field pulses yields

$$
\phi_{2^{\text {nd }}}=-\gamma C Z \delta\left(\frac{Z}{2}-z_{0}-\frac{Z \delta}{2 \Delta}\right)
$$

and evaluation of Eq. 5.41 for the read gradient pulses up until the centre of the acquisition window yields

$$
\phi_{r, 0}=-\gamma g_{R} Z \delta^{\prime}
$$

where $\delta^{\prime}$ is the duration of the dephasing read gradient pulse as illustrated in Fig. 5.6, and is also equal to half the duration of the acquisition window. To determine the total phase due to the read gradients $\phi_{r}$, Eq. 5.41 is evaluated from the centre of the acquisition window until time $t^{\prime}$ for the read gradient, where $t^{\prime}$ can be positive or negative, and is added to Eq. 5.56 to obtain

$$
\phi_{r}\left(t^{\prime}\right)=-\gamma g_{R} Z \delta^{\prime}-\frac{\gamma g_{R} t^{\prime}}{2 \Delta}\left[Z t^{\prime}+2 Z\left(\delta+\delta^{\prime}+\Delta\right)\right]
$$


The total approximate phase $\phi_{t o t}=\phi_{2^{\text {nd }}}+\phi_{r}$, assuming constant velocity and substituting for $Z$ in terms of $t^{\prime}$ using the echo condition from Eq. 5.52, yields

$$
\begin{aligned}
\phi_{t o t}\left(t^{\prime}\right)= & -t^{\prime 3} \frac{\gamma g_{R}^{2}}{2 C \delta \Delta} \\
& -t^{\prime 2} \frac{\gamma g_{R}^{2}\left(3 \delta+2 \delta^{\prime}+3 \Delta\right)}{2 C \delta \Delta} \\
& -t^{\prime} \frac{\gamma g_{R}^{2} \delta^{\prime}}{C \delta},
\end{aligned}
$$

This expression provides the approximate phase correction for each point in time to transform the complex echo signal $E\left(t^{\prime}\right)$ to a real valued function. When the time to displacement mapping from Eq. 5.52 is also applied, and the echo signal $E\left(t^{\prime}\right)$ normalised such that it represents a probability distribution whose integral is equal to unity, the average propagator is obtained without taking the magnitude of the echo signal.

\subsubsection{Conditions for the echo as the average propagator}

For well defined real to $q$-space and time to displacement mappings to exist, the displacement encoded image must be made within the bandwidth of the RF coil, over a homogeneous region of read, second order, $B_{0}$, and RF fields, and the field experienced by the excited slice due to the second order coil must be well approximated by a parabola. Along with a homogeneous sample with constant spin density in the read direction, these conditions ensure the image amplitude at each point in space provides meaningful $q$-space data. For the inverse Fourier transform of this image, i.e. the echo, to represent the average propagator, this displacement encoded image must also experience full attenuation within the homogeneous regions of all magnetic fields.

If the echo is not fully attenuated within the mentioned regions, the resulting image will be the product of some function which modulates the image shape in the absence of the second order pulses, and a Gaussian. For the simplest case, consider a sample of finite length $L$ within the homogeneous regions of the coils, without full attenuation of the image at the edge of the sample. This would yield an image which is the product of a hat function $f(z)=H(|z|-L / 2)=1$ for $-L / 2 \leq z \leq L / 2$, and a Gaussian

$g(z)=\exp \left\{-z^{2} / 2 \sigma^{2}\right\}$ where $\sigma$ relates to the full width at half maximum of the Gaussian and is large enough such that its amplitude is above noise level at $z= \pm L / 2$. The inverse 
Fourier transform of this image, i.e. the echo, can be expressed as

$$
E\left(t^{\prime}\right) \propto \hat{f}(z) \otimes \hat{g}(z)
$$

the convolution of the Fourier transforms of the two functions. In this case, the echo would be the convolution of a Sinc function with a Gaussian, and would not represent the average propagator. When full attenuation down to noise level is achieved at the edge of the sample, the influence of this hat function is no longer apparent in the image. Therefore, the structure of the echo will only relate to the average propagator, and not the spin density.

In the case of the flow propagator measurements presented in this thesis, a pipe of length much greater than the uniformity of the read gradient, slice gradient, and RF fields is used, but has constant spin density. This image, in the absence of the second order pulses, will appear warped due to the effects of these non-uniformities. If, however, the displacement encoded image is formed such that the image is attenuated down to noise level within the uniform regions of these fields, the echo will not be a convolution of the average propagator and the inverse Fourier transform of a function defining the image warping, but will accurately represent the average propagator itself.

With an increasing value of $C$ to ensure full attenuation of the read image within the uniform regions of the coils, for a given slice thickness, the parabolic field approximation will become less and less accurate. This is because for a given slice thickness, larger gradient strengths will be experienced by the edge of the slice as $C$ is increased, and the approximation of $B_{z}=C\left(x^{2}-z^{2}\right) / 2 \approx-C z^{2} / 2$ becomes less accurate. Therefore, for an increasing second order pulse amplitude, or increasing $C$ to ensure full image attenuation at the edge of the image, thinner and thinner slices will be necessary to ensure the parabolic field approximation holds. Only with all the before mentioned conditions will the real space to $q$-space, and therefore the time to displacement space mapping, be well defined, and the echo represent the average propagator. 


\subsection{9 $T_{2}^{*}$ Considerations}

Although great advances have been made in producing NMR magnets with high uniformity, there will always be inhomogeneities in the field which cause dephasing of the spin ensemble. This effect is seen in the free induction decay (FID), which is the initial NMR signal following the first $90^{\circ}$ excitation pulse (Fig. 5.8). Once a $180^{\circ}$ is applied at time $t=\tau$ and the accumulated phases are inverted, these inhomogeneities will begin to rephase the spin ensemble, forming a Hahn echo at time $t=2 \tau$ (Fig. 5.8). The decay

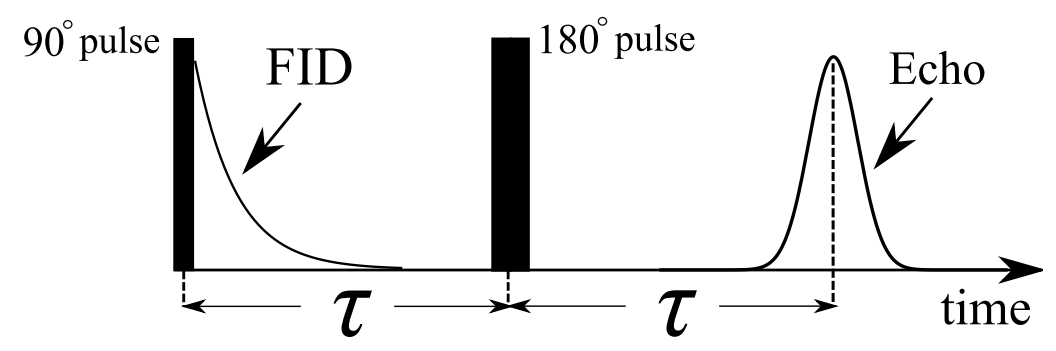

Figure 5.8: A Hahn echo pulse sequence. The FID signal occurs directly after the initial $90^{\circ}$ excitation pulse, and decays in time due to $B_{0}$ field inhomogeneities with a characteristic exponential decay time of $T_{2}^{*}$. At time $\tau$ the $180^{\circ}$ pulse inverts the accumulated phases, and the field inhomogeneities begin to rephase the spin ensemble. At time $2 \tau$ a Hahn echo is formed.

rate of the FID is characterised by the time constant $T_{2}^{*}$. The smaller the value of $T_{2}^{*}$, the greater the inhomogeneities in the static magnetic field, and the faster the FID signal decays. When the spin ensemble is refocused, the Hahn echo $E_{\text {Hahn }}\left(t^{\prime}\right)$ will be composed of back to back FIDs. The width of the Hahn echo is also related to the time constant $T_{2}^{*}$.

The shape of $E_{\mathrm{Hahn}}\left(t^{\prime}\right)$ will act as an amplitude envelope for any further modification of the echo signal. For this reason, care must be taken to ensure the echo $E\left(t^{\prime}\right)$ formed from the pulse sequence in Fig. 5.6 occurs during a region in time where the Hahn echo has a relatively constant magnitude. If this is not the case, the average propagator will be significantly modulated by the amplitude envelope presented by the Hahn echo such that

$$
E\left(t^{\prime}\right) \propto \bar{P}(Z, \Delta) E_{\text {Hahn }}\left(t^{\prime}\right)
$$


For such a scenario, a reference scan must be taken to determine $E_{\text {Hahn }}\left(t^{\prime}\right)$ such that the average propagator can be determined from $E\left(t^{\prime}\right)$.

\subsection{Conclusions}

This chapter has presented a method and theoretical considerations for the parallel acquisition of $q$-space. By combining imaging techniques with a PGSE sequence and encoding for displacement with a second order magnetic field, a one dimensional image of the sample can be mapped from real space to $q$-space, and the echo signal can be mapped from time to displacement space. With this spin echo signal, all relevant displacement information is obtained in a single scan, and the echo itself represents the average propagator. This chapter has also presented a method for the measurement of the diffusion coefficient at multiple observation times in a single scan experiment. This is a new feat which enables a single-shot surface to volume ratio or tortuosity measurement for a porous medium, is order of magnitude faster than other available NMR methods for this measurement, and can therefore resolve this quantity in systems which are evolving on a much faster time scale. Another significant result of this technique is that the observation times for all diffusion measurements are fully correlated back to the same initial event in time, i.e. the first second order field pulse used to encode for displacement.

To implement the techniques presented in this chapter new hardware is required to produce the necessary magnetic fields. In the following chapter design, fabrication, and experimental considerations are discussed in detail, and the necessary gradient coil hardware developed. 


\section{Chapter 6}

\section{Coil Fabrication \& Characterisation}

\subsection{Introduction}

The design and fabrication of custom hardware to apply magnetic fields which vary in space and time are what enables these new experimental techniques to be performed. To implement these new experimental techniques, a second order magnetic field and two first order fields are required. The proposed experiments are performed on two separate systems, with the experimental results presented in chapter 7 . For the low field system (0.05 $\mathrm{T}$ Halbach array) where $\mathbf{B}_{0}$ lies along the $y$-axis, an entirely new gradient stack containing two first order magnetic field coils and a second order magnetic field coil was required. For the high field system (1.5 T superconductor) where $\mathbf{B}_{0}$ lies along the $z$-axis, a three axis imaging gradient stack was already available, and only an insert containing a second order field coil was necessary. The process of building these components occurred entirely in the lab. In this chapter the design considerations, fabrication process, and characterisation are discussed in detail. 


\subsection{Design Considerations for Magnetic Field Gra- dient Coils}

\subsubsection{Introduction to Design Considerations}

The field produced by an electromagnetic gradient coil must obey Maxwell's equations. At the site of the sample in an NMR system where the field is to be generated, it is safe to assume that in the absence of quickly changing fields, the displacement current will obey $\partial \mathbf{D} / \partial t=0$, and the current density $\mathbf{J}=0$ as no current carrying conductors would be present. This results in the relations

$$
\nabla \cdot \mathbf{B}=0
$$

and

$$
\nabla \times \mathbf{B}=0
$$

Taking the cross product of Eq. 6.2 yields

$$
\nabla \times \nabla \times \mathbf{B}=\nabla(\nabla \cdot \mathbf{B})-\nabla^{2} \mathbf{B}=0
$$

Substituting Eq. 6.1 into Eq. 6.3,

$$
\nabla^{2} \mathbf{B}=0
$$

showing the magnetic field for these conditions will obey Laplace's equation. This is a convenient result for design as it allows the use of Laplace eigenmode solutions, such as spherical harmonics, to design electromagnetic coils for use in NMR.

Electromagnetic gradient coils have a long history whose design using spherical harmonics dates back to the 1950's [108-110]. Any magnetic field may be decomposed into an orthogonal set of spherical harmonics, when superimposed on one another, represent the original field. Considering only the $z$-component of the magnetic field in an NMR magnet, 
a solution to Eq. 6.4 may be written as

$$
B_{z}(x, y, z)=\sum_{n=0, m=0}^{\infty} r^{n} P_{n}^{m}(\cos \theta) \times\left[a_{n, m} \cos \{m \varphi\}+b_{n, m} \sin \{m \varphi\}\right],
$$

where $r, \theta$, and $\varphi$ are spherical coordinates, $P_{n}^{m}(\cos \theta)$ are the associated Legendre polynomials of order $m$ and degree $n$, and $a_{n, m}$ and $b_{n, m}$ are coefficients. The idea behind the use of spherical harmonics in NMR is to design coils producing pure magnetic field spherical harmonics, which can systematically shim out unwanted magnetic field spherical harmonics causing inhomogeneities in the $\mathbf{B}_{0}$ field. This same technique may be used to create a coil which produces a highly pure first order spherical harmonic which represents a constant, non-zero magnetic field gradient in space, and also a second order spherical harmonic producing a second order magnetic field. The design of such coils is constrained by the magnet in which it resides, as well as limitations in experimental application such as coil inductance and the power supplies used to drive them.

The first set of physical limitations in coil design are those imposed by the NMR magnet for which it is intended. Normally, gradient coils are composed of wire wound onto, or patterned copper sheets on the surface surrounding the sample inside the NMR magnet. This provides a size constraint since NMR magnets are designed to produce a high field, and consequently do not harbour much free space inside their bore. The spatial constraints determine the maximum efficiency $e$ of the gradient coil, which is given by the gradient per amp $(g / A)$ per square root of the coil resistance such that

$$
e=\frac{g / A}{\sqrt{R}}
$$

given all the available space is used. Once this is determined, the diameter of the wire or thickness of copper sheet is chosen such that the resistance of the coil presents an ideal load for the chosen amplifier to drive against, usually 1-2 $\Omega$.

The sample volume over which the NMR magnet is designed to produce a strong homogeneous field also influences the design of the gradient coil since the gradient coil must produce the desired field within some tolerance or uniformity over the same volume. In 
addition to these spatial restraints on the coil size, the NMR magnet usually offers conductive surfaces over which eddy currents may be induced when the gradient coil is pulsed with current. In an attempt to create a higher gradient field at the sample with higher currents, strong eddy currents may be induced and persist after the coil is switched on or off. If a signal is to be acquired before these eddy currents have dissipated, the field at the sample may be altered to the point where the signal is no longer meaningful.

Further constraints on gradient coil design include those inherent to the coil and those by power sources which will drive the coil. For many experiments, high gradient fields are desirable and may be attained through a large number of turns on the coil, resulting in a high rating of gradient per amp, and also by driving the coil with a high current. When using a coil with a large number of turns, the inductance of the coil also becomes large. This inductance will limit the ability of the current supply to switch it on and off cleanly and abruptly. Switching times of the gradient coil between current levels can be slowed by high inductance and amplifier limitations, which become a hindrance for many pulse sequences, especially fast imaging experiments. The current supplies used to drive the coil also impose limitations as high level current pulses may be difficult to reproduce and match with precision. Precautions must be taken to alleviate this problem by tailoring the current pulses through controlled ramping of the current amplitude. This helps prevent distortions in pulse shapes due to rise and fall delays or oscillations, creating pulses which are more predictable and reproducible.

All these restrictions on design, and consequences of design, present a complex, interdependent parameter space. In an attempt to create a higher gradient field through higher current pulses, eddy currents and switching times can impose time restrictions, and matching of gradient pulses can become difficult. The spatial restrictions of the NMR magnet dictate the amount of wire which may be fit into the gradient stack, and consequently, this limits the efficiency of the gradient coils. In an attempt to fit more turns of into a gradient coil to produce a higher rating of gradient strength per amp, thinner wire may be used resulting in a higher resistance which the current supply may not be suited to drive. All of these considerations result in a delicate balance between attaining the experimental requirements of the gradient coils, while working within the physical limitations imposed by the magnet and the current supplies, and allowing the 
level of unwanted effects to play a role which is manageable for the pulse sequences and experiments in this research.

\subsubsection{Maxwell's Equations and Magnetic Field Gradients}

The fields produced by magnetic field gradient coils will adhere to Maxwell's equations. To investigate the consequences of Maxwell's equations on the fields required to perform the experimental methods proposed in chapter 5, Eq. 6.1 is evaluated to obtain the relationship

$$
\partial B_{x} / \partial x+\partial B_{y} / \partial y+\partial B_{z} / \partial z=0
$$

To design a $y$-gradient coil for Halbach geometry to vary $B_{y}$ in the $y$ direction such that

$$
\partial B_{y} / \partial y \neq 0
$$

the implications of Eq. 6.7 are that

$$
\partial B_{x} / \partial x+\partial B_{z} / \partial z \neq 0
$$

and it remains that $\partial B_{x} / \partial x$ and $\partial B_{z} / \partial z$ cannot both equal 0 . Therefore, the desired gradient of $B_{y}$ in the $\hat{y}$ direction cannot exist without a non-zero gradient of another component of the magnetic field, namely the concomitant fields. For conventional gradient coils producing first order magnetic fields, the effects of the concomitant fields are sometimes encountered and are well understood [111, 112].

Although this is not an ideal scenario for the use of magnetic gradient fields in NMR, the strong magnetic field of the NMR magnet helps to eliminate the effect of the concomitant components. Considering the total field $\mathbf{B}_{t o t}$ from the NMR magnet and the electromagnetic gradient coil, the total field is expressed as

$$
\mathbf{B}_{\text {tot }}=x \frac{\partial B_{x}}{\partial x} \hat{x}+\left(B_{0}+y \frac{\partial B_{y}}{\partial y}\right) \hat{y}+z \frac{\partial B_{z}}{\partial z} \hat{z} .
$$


Taking the magnitude of Eq. 6.10 yields

$$
\begin{aligned}
\left|\mathbf{B}_{\text {tot }}\right| & =\left[\left(x \frac{\partial B_{x}}{\partial x}\right)^{2}+\left(B_{0}+y \frac{\partial B_{y}}{\partial y}\right)^{2}+\left(z \frac{\partial B_{z}}{\partial z}\right)^{2}\right]^{1 / 2} \\
& =\left[B_{0}^{2}+2 B_{0} y \frac{\partial B_{y}}{\partial y}+\left(x \frac{\partial B_{x}}{\partial x}\right)^{2}+\left(y \frac{\partial B_{y}}{\partial y}\right)^{2}+\left(z \frac{\partial B_{z}}{\partial z}\right)^{2}\right]^{1 / 2} .
\end{aligned}
$$

By expanding Eq. 6.11, and keeping only the first two terms, it can be shown that

$$
\left|\mathbf{B}_{t o t}\right| \approx B_{0}+y \frac{\partial B_{y}}{\partial y}+\frac{1}{2} B_{0}^{-1}\left[\left(x \frac{\partial B_{x}}{\partial x}\right)^{2}+\left(y \frac{\partial B_{y}}{\partial y}\right)^{2}+\left(z \frac{\partial B_{z}}{\partial z}\right)^{2}\right]
$$

For almost all NMR magnets and gradient fields, the condition

$$
B_{0} \gg\left[\left(x \frac{\partial B_{x}}{\partial x}\right)^{2}+\left(y \frac{\partial B_{y}}{\partial y}\right)^{2}+\left(z \frac{\partial B_{z}}{\partial z}\right)^{2}\right]
$$

is satisfied, and it is safe to assume

$$
\left|\mathbf{B}_{\text {tot }}\right| \approx B_{0}+y \frac{\partial B_{y}}{\partial z}
$$

This analysis shows that the concomitant fields for first order magnetic fields can be ignored in most cases. Therefore, for the methods proposed in this thesis, they are ignored with the understanding that their effects are sometimes encountered and are well understood $[111,112]$.

As previously shown in subsection 5.1.2, the concomitant fields for second order magnetic field coils cannot be ignored since they will act along the direction of the $\mathbf{B}_{0}$ field. In an attempt to create a second order magnetic field obeying the relationship

$$
B_{y}=\frac{C}{2} y^{2}
$$

as a result of Eq. 6.4, it was found that this field was impossible to create, but a field of

$$
B_{y}=\frac{C}{2}\left(x^{2}-y^{2}\right)
$$


could be created. This field produces a gradient strength of

$$
g=C \sqrt{x^{2}+y^{2}}
$$

To alleviate the dependence of the gradient strength on the $x$-axis, a slice selection was included for the parallel acquisition of $q$-space presented in subsection 5.2.1. This results in a gradient strength dependent only on the $y$-axis, allowing real space to mapped onto $q$-space, and all $q$-space data to be acquired in parallel. Hence, the inclusion of the slice selection in the proposed method for the parallel acquisition of $q$-space is a result of Maxwell's equations and concomitant fields.

\subsection{Coil Design for Halbach Geometry}

\subsubsection{Design Parameters}

In designing the required gradient coils for this research, many constraints and requirement were taken into account to determine the parameter space within which the design must take place. These include spatial constraints due to the NMR magnet and RF coil, gradient strength requirements associated with the coils, amplifiers, and experiments, as well as uniformity of the field produced by the coils and the sample volume over which the fields must be applied.

To begin the design for a gradient stack, the spatial dimensions within which it resides are first considered. The NMR magnet for which this gradient stack was designed is a $2 \mathrm{MHz}$ Rock Core Analyser (Magritek Ltd). This magnet is an array of permanent magnets which creates a $B_{0}$ field perpendicular to the bore axis. The $122 \mathrm{~mm}$ inner diameter of the magnet bore and $76 \mathrm{~mm}$ outer diameter of the RF coil and its components present the inner and outer constraints for the diameter of the gradient stack as shown in Fig. 6.1. In addition to this, to allow for wiring and mounting of the gradient stack within the magnet, the total allowable length for which the wire of the coils may occupy is 300 $\mathrm{mm}$.

With the spatial dimensions defined, space is allocated to each of the three gradient coils 


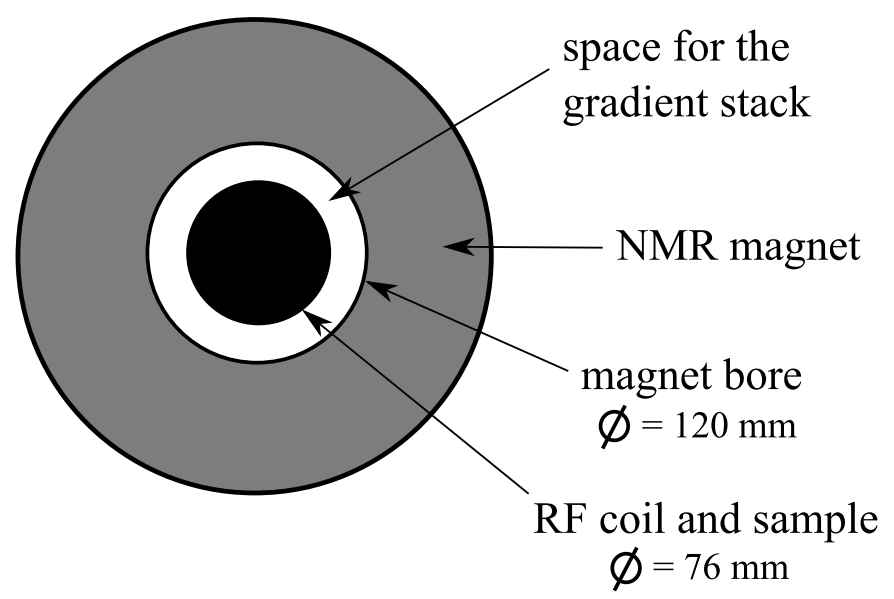

Figure 6.1: Top view of the Halbach NMR magnet. The housing for NMR magnet array is shown in grey, and the space allocated for the RF coil and sample in black. The white area indicates the available space for the gradient stack.

which will reside in the gradient stack. A former, the core upon which the gradient stack is built, was chosen as a fibre glass tube of inner diameter $80 \mathrm{~mm}$, outer diameter $84 \mathrm{~mm}$, and length $385 \mathrm{~mm}$. This former was chosen as it provides a strong, non-magnetic, electrically insulating material which does not emit an NMR signal or harbour eddy currents which will interfere with the experiments. With an outer radial constraint for the gradient stack of $r=60 \mathrm{~mm}$, leaving $1 \mathrm{~mm}$ of radial space around the edge of the stack so it may easily fit into the magnet bore, and an inner radial constraint of $r=42 \mathrm{~mm}, 18 \mathrm{~mm}$ of radial space is left in which three gradient coils must be wound.

In many systems the gradient coils are actively shielded by an extra electromagnetic shielding coil wired in series with the primary gradient coil, and cooled either by air or water flowing through a cooling coil embedded inside the stack. To further determine the amount of space for each layer of the gradient stack, the shielding and cooling requirements for the experiments which this coil is to be used were assessed. Since the pulse sequences presented in chapter 5 do not have strict requirements for signal acquisition to occur directly after the ramping of a strong gradient pulse, eddy currents due to this ramping, which may interfere with signal acquisition, are allowed to be induced in the magnet bore and fade away during a delay period before signal acquisition. In addition 
to this, the bore of this magnet was modified and cut to make the circulation of eddy currents difficult, reducing the lifetime of any eddy currents. Since eddy currents are not of great concern, active shielding was not included in the design. The other component which may take up significant space in the stack is a cooling coil used to dissipate the resistive heating of the gradient stack due to pulsing current through the gradient coils. In the proposed experiments only a single scan is required, and no heavy duty cycle or power dissipation will be placed on the gradient stack, resulting in no need for active cooling of the coil. As a result, only the three unshielded gradient coils were built into the gradient stack, with all $18 \mathrm{~mm}$ of radial space allocated to them.

\subsubsection{First Order Field Requirements}

The requirements of the first order magnetic field gradient coils to be used in this research are determined by demands of the slice selection and read gradient imaging. It is convenient for both first order magnetic field coils to be used interchangeably for slice selection and read images, and were therefore built to the same specifications, first considering the requirements for slice selection.

To determine the requirements for slice selection, the $1 \mathrm{~ms}$ maximum RF pulse duration imposed by the RF amplifier contained in the Kea ${ }^{2}$ spectrometer provided by Magritek Ltd. was first considered. The bandwidth for such a pulse, assuming a rough estimate where the bandwidth is the inverse of the duration, is $1 \mathrm{kHz}$.

The signal amplitude will decrease with decreasing slice thickness, and any slice thinner than some minimum thickness will no longer provide adequate signal to noise for a single-shot measurement. As shown in Fig. 6.2, the signal to volume ratio for water is $1 \mu \mathrm{V} / \mathrm{mL}$. For a $1 \mathrm{~mm}$ thick slice of a cylinder with $\varnothing=35 \mathrm{~mm}$ and height of $60 \mathrm{~mm}$, a total signal amplitude of $2.1 \mu \mathrm{V}$ is obtained. This slice will then be imaged, and therefore this total signal will be distributed among many voxels, making the signal to noise ratio of each voxel equal to the total signal divided by the number of voxels across the selected volume, divided by the rms noise level. For a $1 \mathrm{~mm}$ slice and 16 voxels across the sample, this yields a signal to noise ratio per voxel of only 1.3, and it is safely assumed that any slice $<1 \mathrm{~mm}$ in thickness will produce a signal to noise ratio which is far below the level 

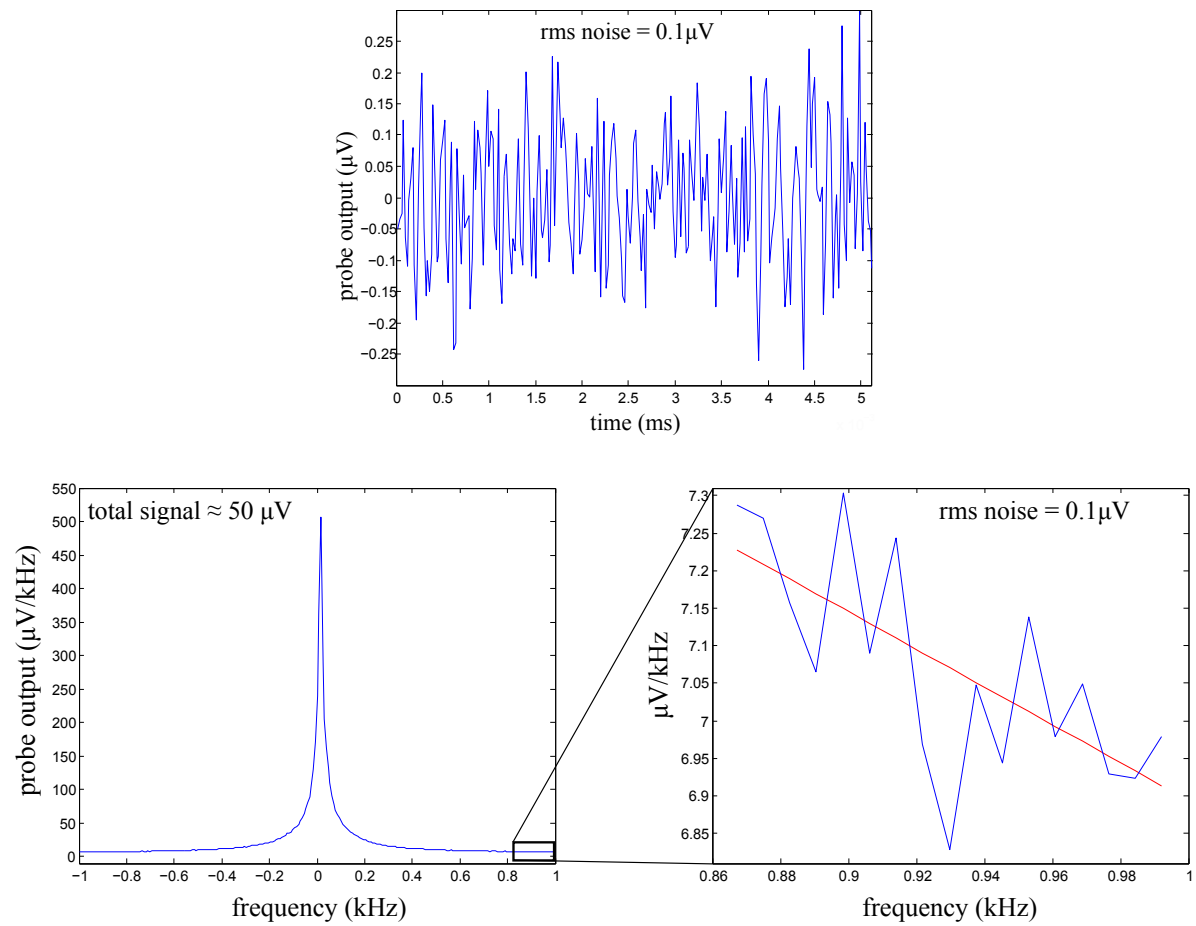

Figure 6.2: Top: Noise scan with $20 \mu$ s dwell time, 256 points. Left: Magnitude of a FID spectrum for $50 \mathrm{~mL}$ of water with a total area of $50 \mu \mathrm{V}$. Right: The tail end of the spectrum is magnified and a linear fit is applied. The rms noise is calculated with respect to this fit showing the noise level still agrees with the previous noise measurement of $0.1 \mu \mathrm{V}$.

necessary for a single-shot technique. Taking an overestimate in the thinness for slice selection of $1 \mathrm{~mm}$, and using a $1 \mathrm{kHz}$ bandwidth for the soft pulse, the gradient necessary to obtain such a slice is calculated using the relationship

$$
B W=\gamma g \Delta x
$$

where $B W$ is the bandwidth of the RF pulse, $g$ is the gradient strength in Tesla per meter, and $\Delta x$ is the slice thickness. This yields a gradient strength of $g=23.5 \mathrm{mT} / \mathrm{m}$. This determines the total gradient strength necessary for such a slice selection. For the coil design a requirement must be defined in gradient per amp. Since these gradient coils will be driven by B-AFPA 40 amplifiers from Bruker, the maximum stable current 
available is $40 \mathrm{~A}$. Therefore, the gradient requirement of the first order magnetic fields for slice-selection will be

$$
g / A=.0235 / 40=0.588 \mathrm{mT} / \mathrm{m} / \mathrm{A}
$$

where the rating is in units of milli-Tesla per meter per amp.

The limiting factors for the read gradient are the linewidth of the NMR magnet, the bandwidth of the RF receiver coil, and the bandwidth of the spectrometer. When performing a read image, the signal must be spread over a bandwidth $B W$ such that $B W \gg \Delta f_{0}$, where $\Delta f_{0}$ is the bandwidth of the frequencies produced across the sample due to inhomogeneities in the $B_{0}$ field. For a sample diameter and length of $\varnothing=35 \mathrm{~mm}, l=60$ $\mathrm{mm}$, this $2 \mathrm{MHz}$ NMR magnet produces an unshimmed linewidth of 40 parts per million, or $\Delta f \approx 80 \mathrm{~Hz}$. Although shimming with the gradient stack will produce a narrower linewidth as it reduces the amount of inhomogeneity in the $B_{0}$ field, it is not known to what degree it will do so until after it is built and used to shim. Making a safe assumption that the bandwidth of signal from the sample composing the one dimensional read image should satisfy $B W>100 \Delta f$, it is determined that $B W>8 \mathrm{kHz}$. This bandwidth is less than the $25 \mathrm{kHz}$ bandwidth of the RF coil, and well within the bandwidth of the spectrometer. Again, using the relationship from Eq. 6.18, a 40 A max current, and the sample diameter, a rating for the coil is found such that

$$
g / A=\frac{100 \Delta f}{\gamma \varnothing I_{\max }} \approx .134 \mathrm{mT} / \mathrm{m} / \mathrm{A} .
$$

The requirement from slice selection of $g / A>0.588 \mathrm{mT} / \mathrm{m} / \mathrm{A}$ is more demanding than this requirement for the read gradient, and is therefore used as the minimum gradient rating for both first order magnetic fields.

The final constraint in the requirements for the first order magnetic field gradient coils is that of gradient strength uniformity in space. To help prevent misshapen slices or skewed and distorted read images, the percent deviation of the gradient strength over the entire sample with respect to the gradient strength at the centre of the sample should not exceed a given tolerance. The sample is again assumed to possess a diameter of $\varnothing=35 \mathrm{~mm}$, and 
height of $60 \mathrm{~mm}$. As a rule of thumb, and a common tolerance in commercial gradient coils, the uniformity of the gradient strength should be within $5 \%$ deviation over the entire volume of the sample.

\subsubsection{Second Order Field Requirements}

The second order coil is used to encode for $q$-space, and in most of the experiments, induce diffusive attenuation. This coil will produce a parabolic magnetic field profile when used in conjunction with slice selection, and it is preferable that the gradient strength varies as linearly as possible with respect to position along the direction of the read gradient. The range of gradient strengths experienced across the sample should provide a full acquisition of the relevant $q$-space. This means that at the edges of the sample where the gradient will be largest, diffusive attenuation should occur all the way down to the noise level. This will yield the full range of relevant $q$-space, with the highest applied $q$-value relating to the smallest resolvable displacement.

To determine the level of diffusive attenuation necessary to reach the noise level, the signal per $\mathrm{mL}$ of water is determined by taking the spectrum of an FID for $50 \mathrm{~mL}$ of water. The spectrum is integrated and then divided by the volume of water used, giving the signal per volume of water equal to $1 \mu \mathrm{V} / \mathrm{mL}$. The slice thickness for the proposed experiments is then overestimated as $10 \mathrm{~mm}$. Using the same sample dimensions for analysis as in subsection 6.3.2, this gives an overestimate of the amount of signal which will need to be attenuated down to the noise level, and is found to be a total signal of $21 \mu \mathrm{V}$, or $1.3 \mu \mathrm{V} /$ point assuming 16 voxels across a read image of the slice.

To find the necessary gradient strength for diffusive attenuation of this signal down to noise level, the normalised signal amplitude as a function of gradient strength is used. Taking the logarithm of Eq. 4.40, and using the realtionship $q=\gamma \delta g$, the amount of diffusive attenuation can be expressed as

$$
\ln \left[E_{N}(g, \delta, \Delta)\right]=-D \gamma^{2} g^{2} \delta^{2}(\Delta-\delta / 3)
$$


Here, $E_{N}$ is determined using the normalised signal for diffusive attenuation down to noise level such that

$$
E_{N}=\frac{E_{\text {noise }}}{E_{\text {sig }}}=\frac{0.1 \mu \mathrm{V}}{1.3 \mu \mathrm{V}}=0.0769 .
$$

Substituting $E_{N}$ into Eq. 6.21,

$$
-2.56=-D \gamma^{2} g^{2} \delta^{2}(\Delta-\delta / 3)
$$

The curvature of the second order field $C$ characterises the field and the rate of change of the gradient strength. Letting $g / A=C y$ where $A$ is the maximum available current from the B-AFPA 40 amplifier, $y$ is the position at the edge of the sample, and dividing both sides of Eq. 6.23 by the maximum available current of $40 \mathrm{~A}$, the condition for attenuation down to noise level at the edge of the sample becomes

$$
-0.0016 \times 10^{-4} A^{-2}=-D \gamma^{2}(C y)^{2} \delta^{2}(\Delta-\delta / 3) .
$$

Assuming the diffusion coefficient of water at room temperature $D=2 \times 10^{-9} \mathrm{~m}^{2} / \mathrm{s}$, a gradient pulse length of $\delta=5 \mathrm{~ms}$, an observation time of $\Delta=30 \mathrm{~ms}$, and the gyromagnetic ratio of protons as $42.57 \mathrm{MHz} / \mathrm{T}$ such that $\gamma=2 \pi \times 42.57 \times 10^{6}$, the necessary gradient strength per amp at the edge of the sample is determined to be $C y \approx 4.0 \mathrm{mT} / \mathrm{m}$. For a $35 \mathrm{~mm}$ diameter sample, the edge of the sample is found at $y=17.5 \mathrm{~mm}$. Solving for $C$ for the given experimental parameters and geometry, a minimum value for the coil parameter $C$ is determined such that

$$
C>0.23 \mathrm{~T} / \mathrm{m}^{2}
$$

\subsubsection{Modelling Gradient Coil Characteristics}

The two linear magnetic field coils will easily meet requirements and will be designed after the more demanding second order coil specifications are met. The magnetic field gradients produced by the second order magnetic field coil will be strongest for a coil which is closest to the sample, so this coil is designed such that it will be the first wound 
onto the former.

A $\sin \{2 \theta\} \hat{z}$ current distribution will create a $1^{\text {st }}$ order $B_{y}$ field, and a current distribution of $\sin \{3 \theta\} \hat{z}$ will produce a $2^{\text {nd }}$ order $B_{y}$ field. To approximate these current distributions, straight wire segments running along the axial direction of the former may be used. Wires wound along the length of the former spaced $\pi / 3$ radians apart, with alternating direction of electrical current flow, approximates the desired $\sin \{3 \theta\} \hat{z}$ current distribution required for a second order $B_{y}$ field.

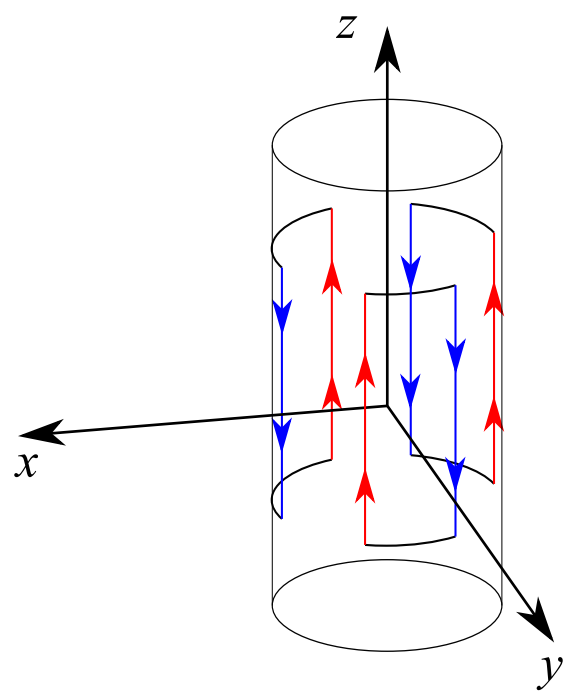

Figure 6.3: Wiring pattern for an $B_{y}=\frac{C}{2}\left(x^{2}-y^{2}\right)$ coil. Loops of wire are spaced such that a $\sin \{3 \theta\} \hat{z}$ current distribution is approximated.

Here it is noted that for fabrication purposes, these lengths of wire may not be infinitely long and must join one another at some stage such that there is a single input and output connection for the entire coil. The lengths of wire which connect the poles will alter the desired magnetic field at the sample, and there will also exist lengths of wire connecting the loops shown in Fig. 6.3. The magnetic field resulting from the connecting paths between the three loops may be cancelled out by the final return path of the wire running directly along side of it to produce a local current density which approaches zero. Choosing a radius of $42 \mathrm{~mm}$ such that the wires lie on the surface of the former, and a loop length of $250 \mathrm{~mm}$ along the axis of the former, the magnetic field produced by a 

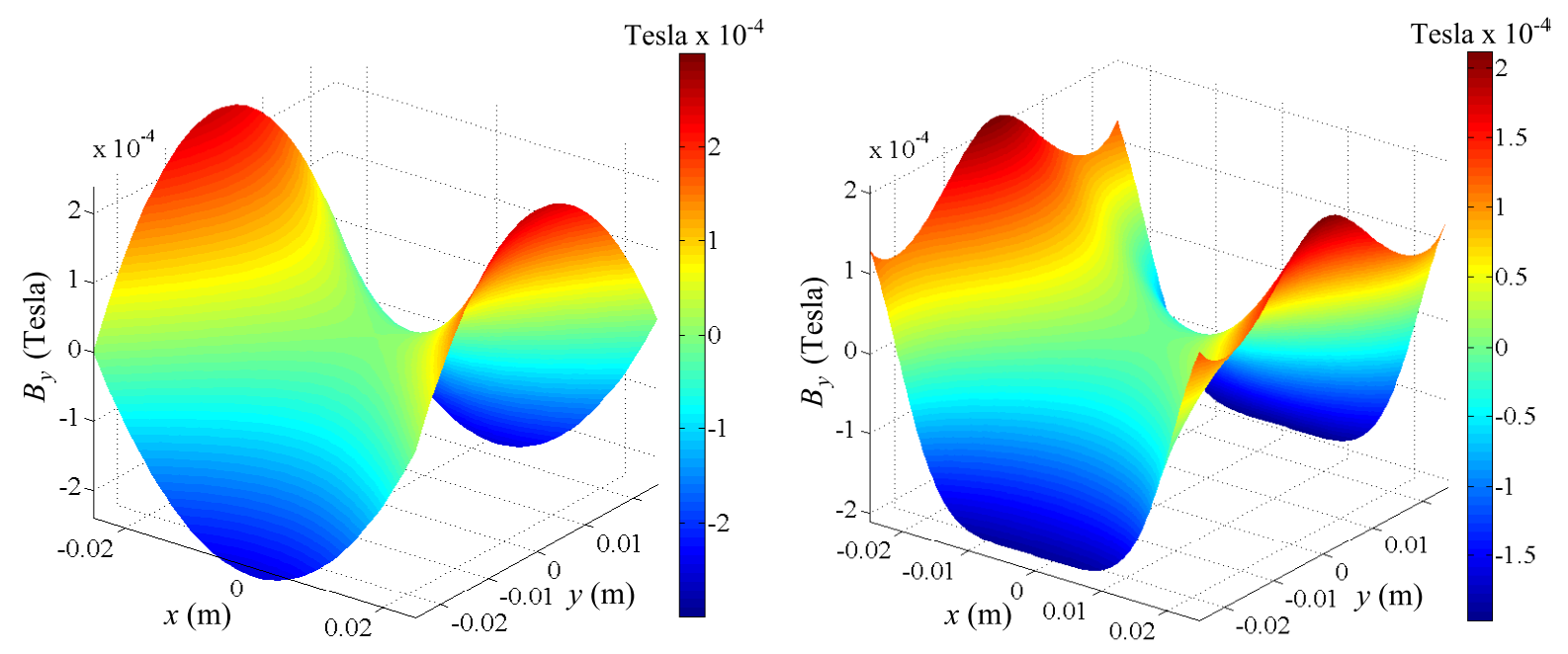

Figure 6.4: Calculated magnetic fields. Left: Ideal field in the $x y$-plane where $B_{y}=$ $\frac{C_{0}}{2}\left(x^{2}-y^{2}\right)$. Right: $B_{y}$ field in the $x y$-plane resulting from the wiring pattern specified in Fig. 6.3 using 25 turns of wire. Note: $C_{0}$ for the figure on the left is taken as the gradient per meter at the center of the sample from the figure on the right.

single turn of wire along the three loops is modelled. Using the Biot-Savart law

$$
\mathbf{B}=\frac{\mu_{0}}{4 \pi} \int_{P} \frac{I d \mathbf{l} \times \mathbf{r}}{|\mathbf{r}|^{3}}
$$

where $\mu_{0}$ is the vacuum permeability and the line integral is taken over the path of the wire $P$, a magnetic field profile (Fig. 6.4) is calculated. The rate of change of the gradient of the $B_{y}$ component of this field is found to be $C /$ turn $\approx 0.027 \mathrm{~T} / \mathrm{m}^{2} \mathrm{~A}$ at the center of the sample. With the minimum specification for $C$ of $0.23 \mathrm{~T} / \mathrm{m}^{2} \mathrm{~A}$, if 25 turns of wire are chosen, this will easily satisfy the already overestimated gradient requirement, setting $C \approx 0.810 \mathrm{~T} / \mathrm{m}^{2} \mathrm{~A}$.

To quantify the deviation of the field produced by the wiring pattern in Fig. 6.3 from the ideal $B_{y}=\frac{C}{2}\left(x^{2}-y^{2}\right)$ field, the uniformity of $C$ is investigated. The uniformity of $C$ is calculated by numerically evaluating the total gradient per meter from the modelled 

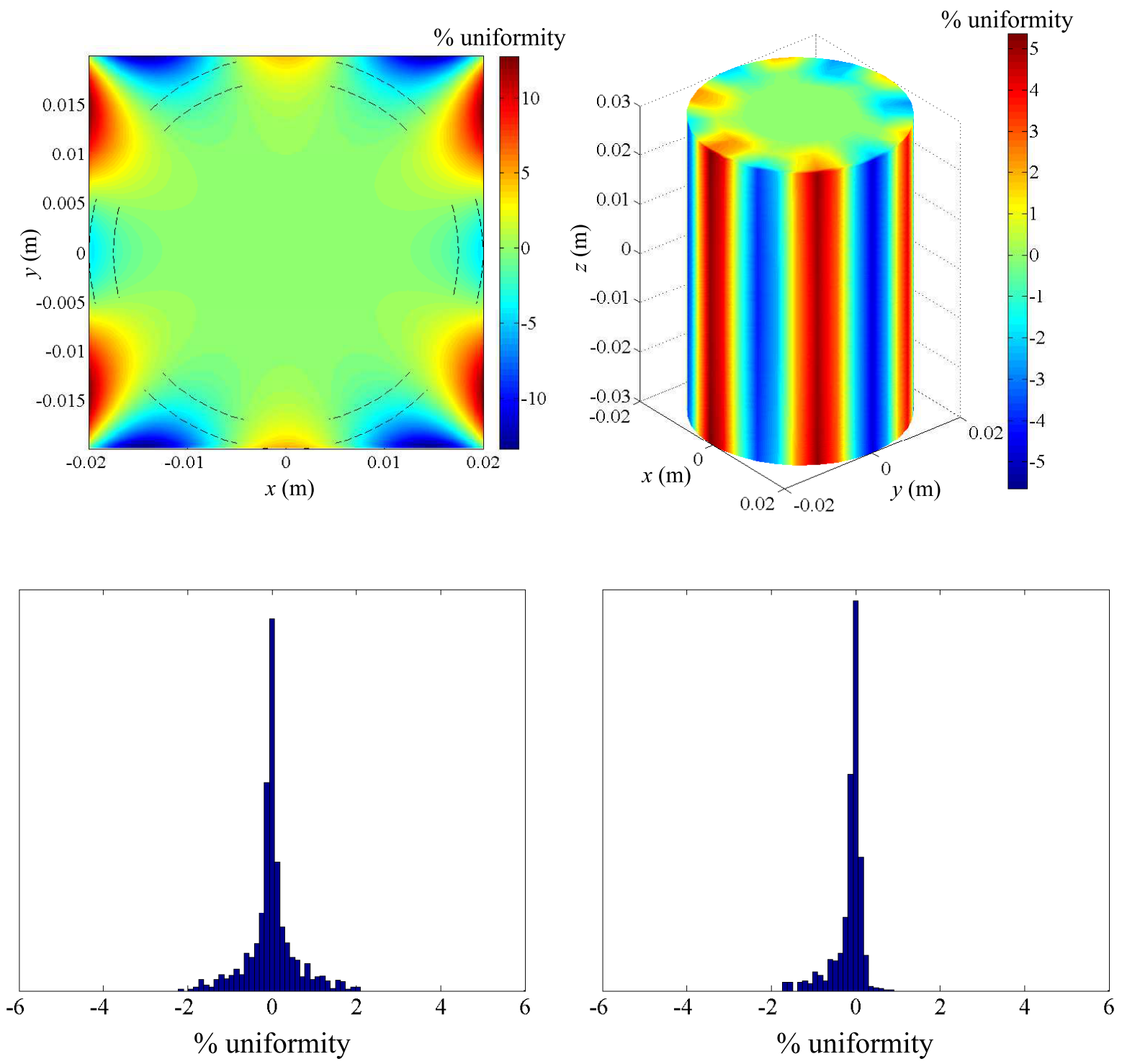

Figure 6.5: Calculated percent uniformity of $C(\mathbf{r})$ with respect to $C_{0}$ over a cylindrical sample volume of $\varnothing=35 \mathrm{~mm}$, length $=60 \mathrm{~mm}$. Top left: Percent uniformity in the $x y$ plane. Top right: Percent uniformity on the surface of the sample volume. Bottom left: Histogram of percent uniformity of $C$ for volume elements of the entire sample volume. Bottom right: Percent uniformity histogram with only a $10 \mathrm{~mm} x$-slice selection volume considered. 
field as a function of position

$$
C(\mathbf{r})=\frac{|\mathbf{g}(\mathbf{r})|}{\sqrt{x^{2}+y^{2}}},
$$

where

$$
\mathbf{g}(\mathbf{r})=\nabla B_{y}(\mathbf{r})
$$

and then finding the percent deviation from the coil rating $C$ taken at the center of the sample

$$
\% \text { Deviation }=100 \times\left(1-C(\mathbf{r}) / C_{0}\right)
$$

As illustrated in Fig. 6.5, the uniformity of $C(x, y)$ is within the $5 \%$ tolerance for the desired sample volume, and the majority of deviation $C(x, y, z)$ from $C_{0}$ occurs at the surface of the sample.

To determine whether 25 turns of wire is a reasonable for this coil, the space required for such a winding is determined for a wire diameter giving a total resistance of the coil $0.5 \Omega \leq R \leq \Omega$, the desired resistance against which the amplifiers should be driving. Calculating the total length of the coil to be $l \approx 44 \mathrm{~m}$, a reasonable wire diameter of $\varnothing_{\text {wire }}=0.67 \mathrm{~mm}$ gives a resistance of $2.15 \Omega$. Although this is at the upper range of the desired resistance, a larger diameter wire would occupy more space in the radial direction, which is undesirable since two first order coils must be wound on top of the second order coil.

Taking spatial restrictions into consideration, the amount of radial space this coil will consume in the gradient stack must be determined. These windings should be placed such that they form a $5 \times 5$ grid on the surface of the former such that all wires reside as close as possible to the position of the modelled wiring pattern, but it can be assumed that this will not occur as they will be hand wound with solid core copper wire. Furthermore, the wire may not be perfectly straight as it is unwound from a spool, resulting in less perfect stacking. Assuming a mean effective wire diameter $\varnothing_{\text {eff }}=1 \mathrm{~mm}$ due to kinks in the wire, and a 7 layer stacking height, a safe assumption is made that $7 \mathrm{~mm}$ of radial space will be consumed by this coil (Fig. 6.6).

Next, the amount of radial space which will be left to design the two first order magnetic 

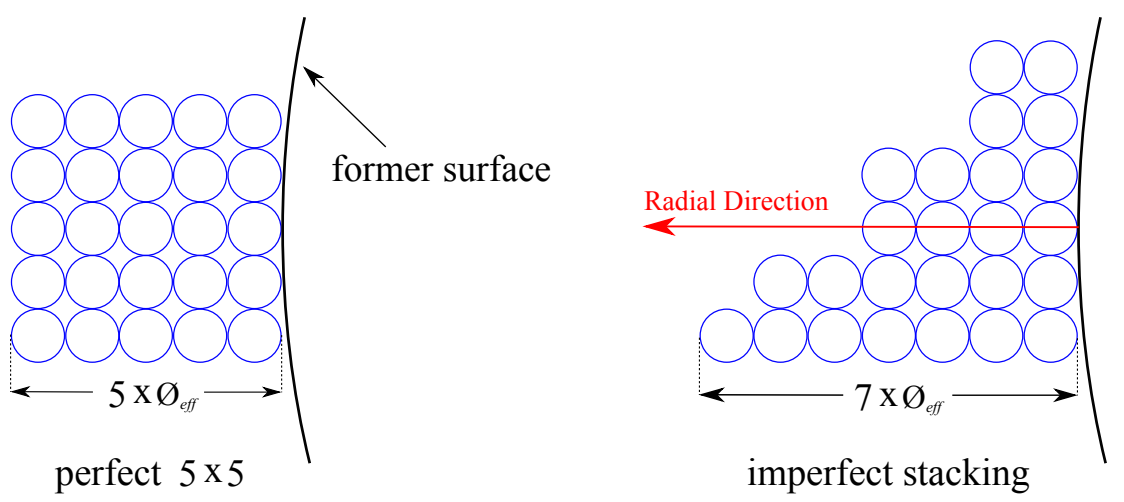

Figure 6.6: Left: An ideal stacking of the 25 required turns for each loop on the second order magnetic field coil. Right: Wire stacking scenario resulting from a more likely, imperfect winding of turns on the coil.

field gradient coils is calculated. Beginning with $18 \mathrm{~mm}$ of radial space, and assuming $7 \mathrm{~mm}$ of radial space is consumed by the second order field coil, allowing a $3 \mathrm{~mm}$ gap between any wire and the surface of the gradient stack, $8 \mathrm{~mm}$ of space remains for the two first order field coils, or $4 \mathrm{~mm}$ per remaining coil. This will put the surfaces which the first order field coils will be wound at radii of $49 \mathrm{~mm}$ and $53 \mathrm{~mm}$.

The next gradient coil in the stack, a first order coil producing an $x$-gradient, should have a current density which varies as $I_{z}(\theta)=\sin \{2 \theta\} \hat{z}$. Using straight lengths of wire spaced $\pi / 6$ radians apart provides a good approximation of the desired current density profile about the circumference of the former.

Using the Biot-Savart calculation to determine the magnetic field produced by the wiring pattern, choosing a loop length of $270 \mathrm{~mm}$ along the axial direction of the gradient stack and a coil radius of $49 \mathrm{~mm}$, a gradient field strength of $g \approx 0.580 \mathrm{mT} / \mathrm{m} / \mathrm{A} / \mathrm{turn}$ is calculated. The coil requirement is only $0.588 \mathrm{mT} / \mathrm{m} / \mathrm{A}$ for this coil, and the amplifier would like to be driving against a resistance of $0.5 \Omega \leq R \leq 2 \Omega$. Therefore the number of turns on this gradient coil is chosen such that the same thickness of wire used for the second order field coil results in a $1 \Omega$ resistance. Using 10 turns of wire for this coil, the resistance is calculated to be $R \approx 1.25 \Omega$ with a gradient rating of $g \approx 5.80 \mathrm{mT} / \mathrm{m} / \mathrm{A}$, and the coil should occupy a maximum of $4 \mathrm{~mm}$ of radial space.

The final gradient coil to be wound on this stack is to produce a first order magnetic 


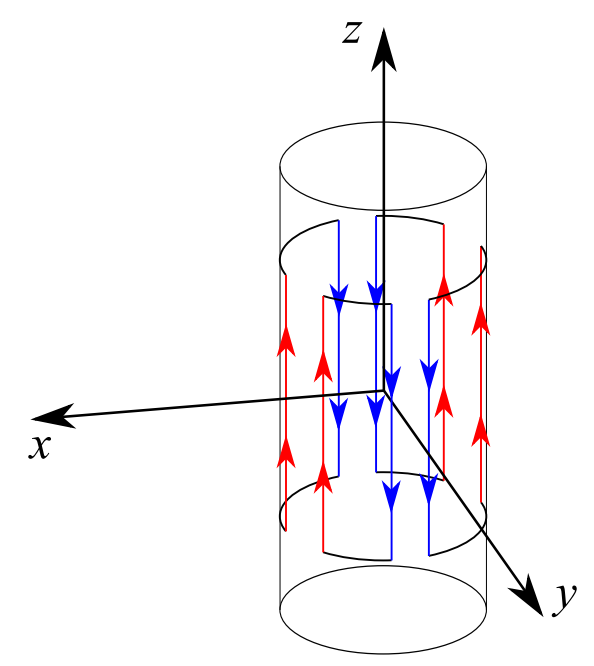

Figure 6.7: Wiring pattern for an $x$-gradient coil for Halbach geometry. Loops of wire are spaced such that a $\sin \{2 \theta\} \hat{z}$ current distribution is approximated.

field and $y$-gradient. Using the same approximation for a $\sin \{2 \theta\} \hat{z}$ current distribution as for the $x$-gradient, the same number of turns and wire diameter, and by rotating the wiring pattern by $\pi / 4$ radians, the design for the $y$-gradient is obtained. Calculating the resistance and magnetic field from this pattern for a coil radius of $53 \mathrm{~mm}$ and loop length of $290 \mathrm{~mm}$, it is found that $R \approx 1.35 \Omega$ with a gradient rating of $g \approx 4.95 \mathrm{mT} / \mathrm{m} / \mathrm{A}$.

To better assess the magnetic fields produced by these coils, the gradient uniformity is evaluated over the sample surface. Since the most extreme deviation from the gradient strength at the center of the sample will occur at the surface of the sample, the surface is used as a worst case scenario for uniformity constraints. The percent deviation of gradient uniformity with respect to the central gradient $g_{0}$ is expressed as

$$
\% \text { Deviation }=100 \times \frac{|\mathbf{g}(\mathbf{r})|}{\left|\mathbf{g}_{0}\right|},
$$

where

$$
\mathbf{g}(\mathbf{r})=\nabla B_{y}(\mathbf{r}),
$$

and $\mathbf{g}_{0}$ is the gradient taken at the center of the sample. As shown in Fig. 6.8, the gradient uniformity for both first order magnetic field coils are well within the desired 

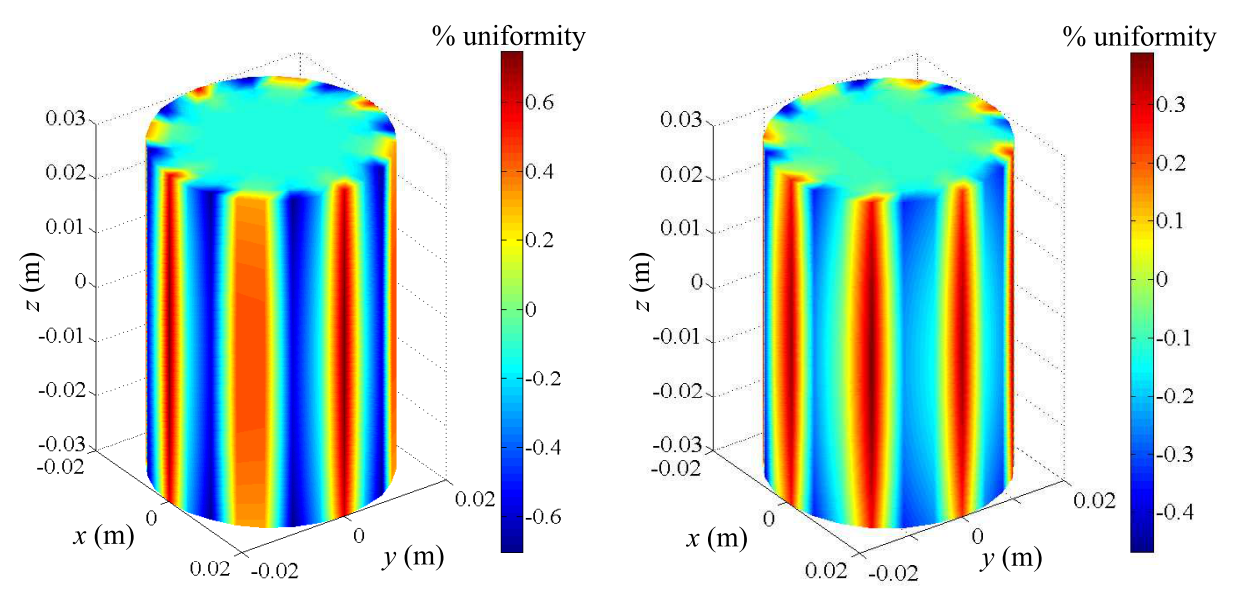

Figure 6.8: Calculated gradient uniformities. Left: Gradient uniformity over a $\varnothing=$ $40 \mathrm{~mm}$, length $=60 \mathrm{~mm}$ sample surface for the $x$-gradient. Right: Gradient uniformity over the same sample surface for the $y$-gradient.

tolerance.

\subsubsection{Fabrication \& Mapping}

The central component of this gradient stack onto which all material will be laid is the gradient coil former. The material chosen for this component is e-glass, a fibre glass material whose fibres are laid down in ordered sheets, impregnated with a resin, and then baked to cure the resin. This material was chosen because the ordered fibres give it more strength than normal fibre glass, and the material itself does not conduct electricity, is non-magnetic, and does not emit an NMR signal which is capable of interfering with the experiments presented in this research due to its extremely short $T_{2}$.

The former was ordered from CTSMI, a company in the yachting industry which manufactures fibre glass and carbon fibre tubes. A tube of outer diameter $84 \mathrm{~mm}$ and inner diameter $80 \mathrm{~mm}$ with a length of $385 \mathrm{~mm}$ was made. This former varied slightly by $\pm 0.2 \mathrm{~mm}$ in diameter.

As shown in Fig. 6.9, the coils to be built are composed of wire loops. The easiest way to wind these loops is to define the points at which the wires bend. This is done by fixing 

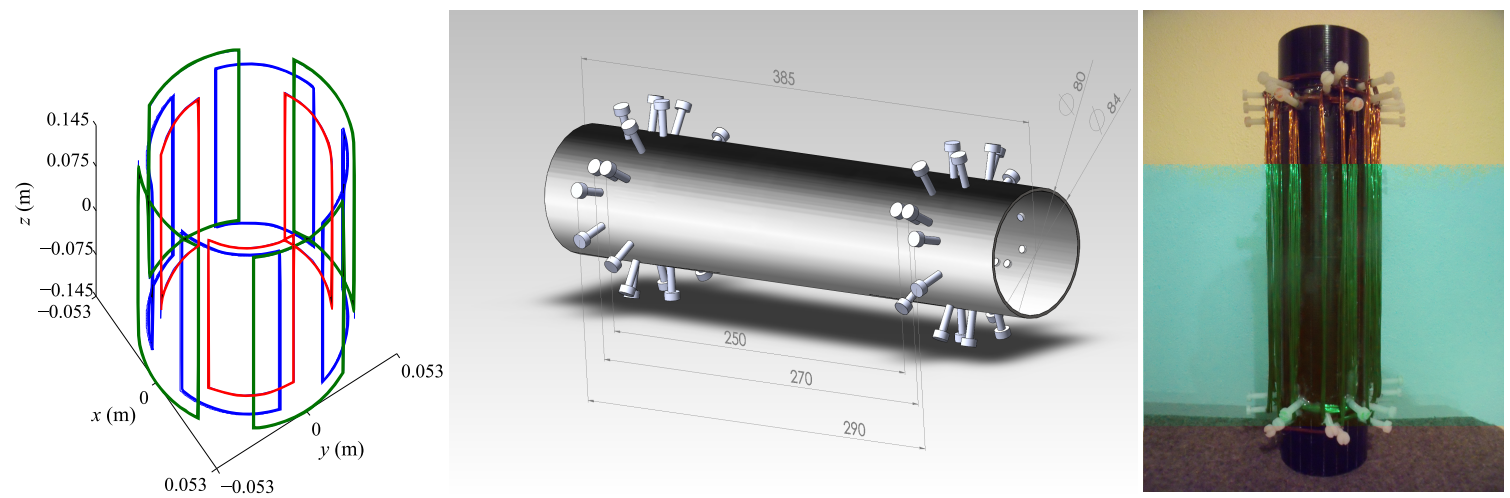

Figure 6.9: Left: Wiring patterns for all 3 gradient coils to be wound in the gradient stack, drawn to scale. The $x^{2}-y^{2}$ coil is drawn in red, the $x$-gradient in blue, and the $y$-gradient in green. Centre: Drawing of peg placement in the former defining the vertices for the coil windings. Right: Fully wound gradient stack.

a pivot point at each of the corners of the loops at the appropriate height and radial distance from the axis of the former. To accomplish this, the former is drilled and tapped such that the edge of each tapped hole corresponds to a vertex of each wire loop to be wound. Nylon screws are then screwed into each hole, giving a pivot for the wiring of each loop as shown in Fig. 6.9.

The coils were then wound starting with the second order magnetic field coil. This first coil was wound tight against the surface of the former, making winding very straight forward. The next coil wound was the $x$-gradient coil. To define its radius from the axis of the former, grooves were cut into the nylon screws at the appropriate distance which the wire was wound into. Lastly, the $y$-gradient was wound, again using grooves cut into the nylon screws to define its radius from the centre of the former.

Before potting the gradient coils in an epoxy resin for thermal and mechanical stability, the magnetic fields they produce are mapped to ensure they have been wound properly. The field mapping was performed by a robotic control arm holding a three axis hall probe, with the magnetic field measured at points on the surface of a sphere. With the magnetic field data for the surface of a sphere, a spherical harmonic decomposition can be used to reconstruct the field for any grid of points whose range is reasonably close to the range over which the spherical field map was acquired. 


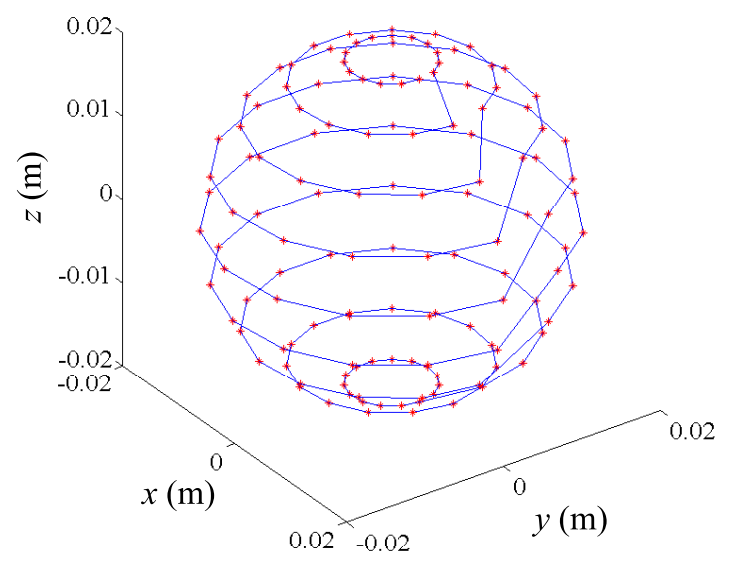

Figure 6.10: Path of the 3 axis hall probe shown in blue, with the coordinates for magnetic field measurements shown in red.

To acquire the field map on the surface of a sphere, a list of coordinates is loaded into the Prospa software (Magritek Ltd.), and used to control the robotic mapper. A DC current of $2 \mathrm{~A}$ is run through the coil while the probe records the resulting magnetic field components at each coordinate. The path of the hall probe and positions for data acquisition are shown in Fig. 6.10.

Taking the spherical harmonic decomposition of the $B_{y}$ component of the magnetic field, the field is reconstructed over a grid with a range of $-20 \mathrm{~mm}<x<20 \mathrm{~mm}$, $-20 \mathrm{~mm}<y<20 \mathrm{~mm}$, and $-30 \mathrm{~mm}<z<30 \mathrm{~mm}$. The field is then interpolated for the surface of a cylindrical sample, and analysis of the field for each coil is performed.

For the second order magnetic field coil, Fig. 6.11 shows the $B_{y}$ component of the magnetic field in the $x y$-plane and the uniformity of $C$ over the surface of a cylindrical sample with radius $20 \mathrm{~mm}$ and length $60 \mathrm{~mm}$.

It was found that the rating of $C_{0} / \mathrm{A}$ is $0.731 \mathrm{~T} / \mathrm{m}^{2} \mathrm{~A}$, slightly less than the modelled value of $0.810 \mathrm{~T} / \mathrm{m}^{2} \mathrm{~A}$, but more than the required $0.23 \mathrm{~T} / \mathrm{m}^{2} \mathrm{~A}$. This may be attributed to the fact that the windings are actually a stacked array of 25 wires at varying radii and angles about the modelled wiring pattern, but were modelled as a single wire with $25 \mathrm{~A}$ of current. The most important information extracted from this mapping process is that the uniformity of $C$ appears to be better than the modelled uniformity, with a maximum 

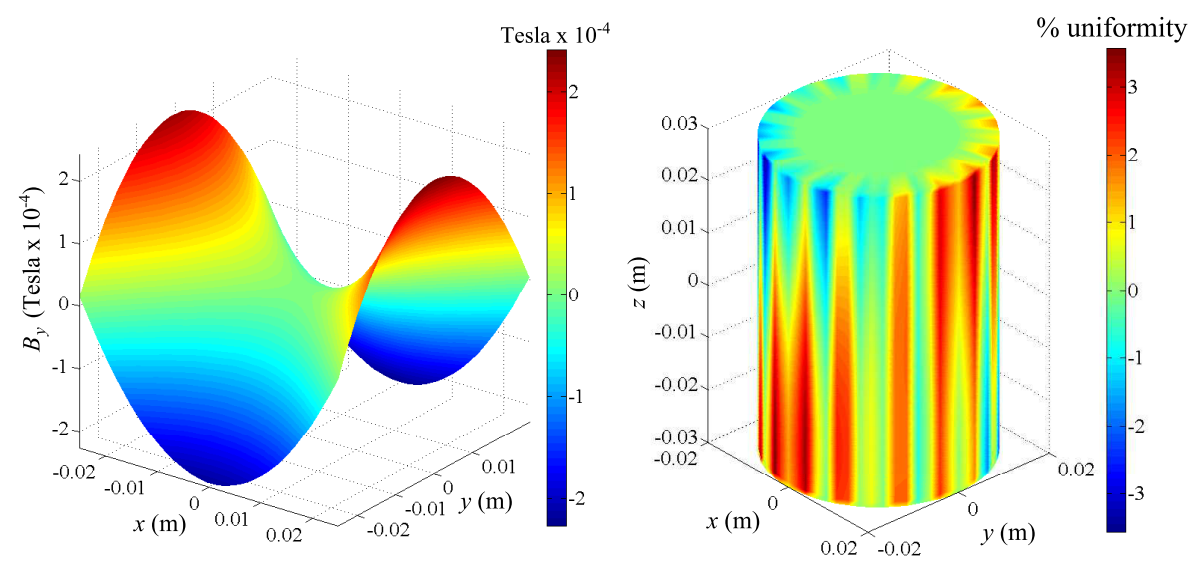

Figure 6.11: Analysis of the field mapping data for the second order magnetic field gradient coil. Left: $B_{y}$ in the $x y$-plane. Right: Uniformity of $C$ over a cylindrical sample surface of radius $20 \mathrm{~mm}$ and length $60 \mathrm{~mm}$.

deviation from $C_{0}$ of less than $4 \%$ over the $20 \mathrm{~mm}$ radius volume, making this field fit for experimental purposes.

Next, the field maps of the first order magnetic field gradient coils were taken. The same mapping approach was used as for the second order magnetic field coil, but this time the gradient strength and its uniformity are analysed (Fig. 6.12). It was found that for the $y$-gradient, the gradient strength at the center of the sample is $5.58 \mathrm{mT} / \mathrm{m} / \mathrm{A}$, stronger than the modelled $4.95 \mathrm{mT} / \mathrm{m} / \mathrm{A}$. This is attributed to the windings positioned at a smaller radius than modelled. It was also found that the gradient uniformity varies between $-2.2 \%$ and $+3.5 \%$ for a $\varnothing=40 \mathrm{~mm}$ sample, where the modelled uniformity predicted values between $-0.5 \%$ and $+0.4 \%$ for a $\varnothing=35 \mathrm{~mm}$ sample. This is also, in part, attributed to the windings located at a smaller radius, but more likely due to the stacking of the wires resulting in random variation in their position, and the resulting current density distribution deviating from the desired current density.

For the $x$-gradient much greater deviation from the modelled field is found upon mapping the field. The gradient strength at the center of the sample is measured to be $6.13 \mathrm{mT} / \mathrm{m} / \mathrm{A}$, stronger than the predicted $5.8 \mathrm{mT} / \mathrm{m} / \mathrm{A}$. This may again be attributed to the windings sitting at a radius smaller than the modelled value. It was also found that 

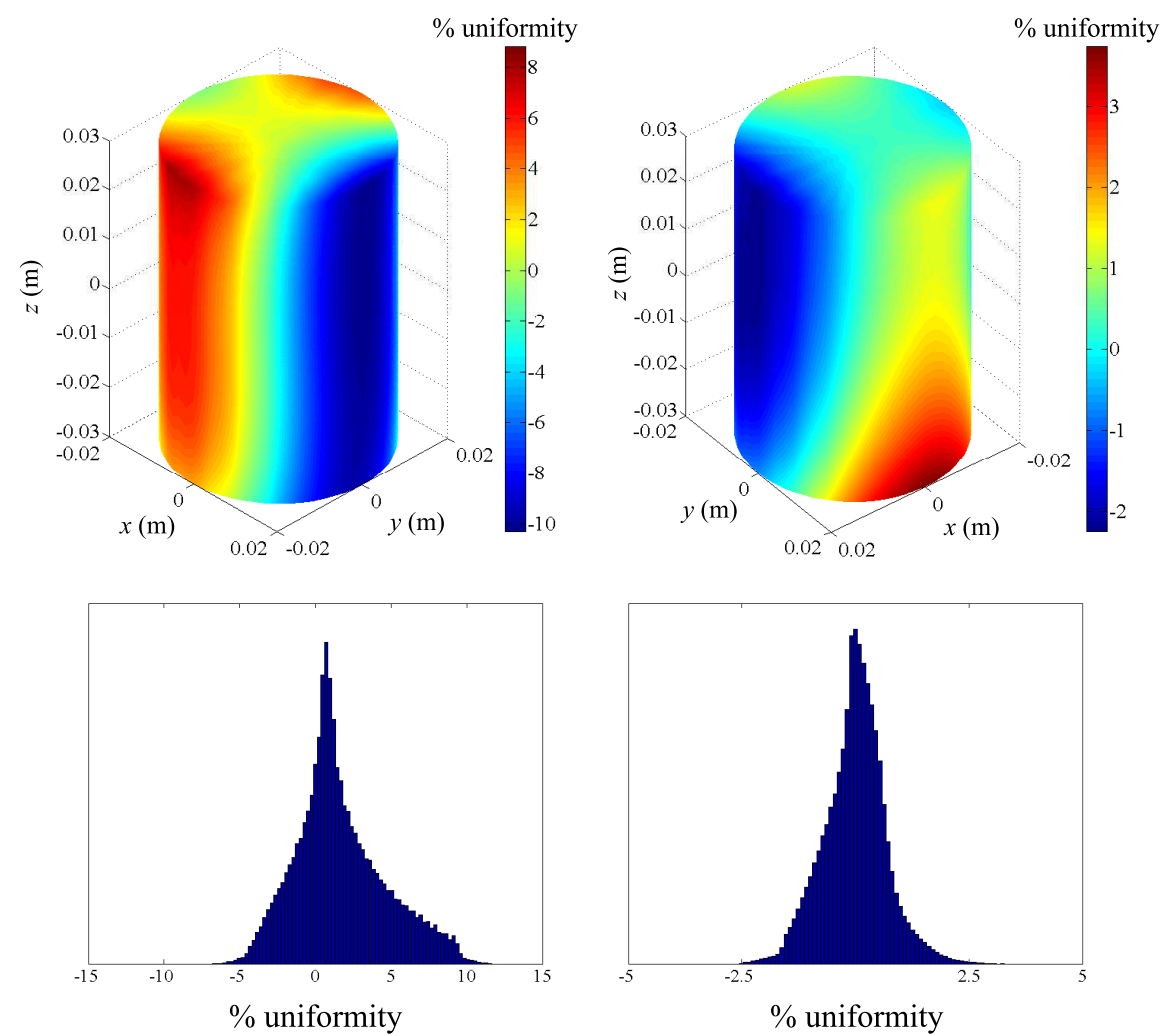

Figure 6.12: Measured gradient uniformity maps for the $x$ and $y$-gradient coils. Left: $x$-gradient percent uniformity over a $\varnothing=40 \mathrm{~mm}$, length $=60 \mathrm{~mm}$ sample surface and histogram over the sample volume. Right: $y$-gradient uniformity analysis for the same sample size.

the uniformity is far worse than modelled range of $-0.7 \%$ to $+0.7 \%$ for a $\varnothing=35 \mathrm{~mm}$ sample, and instead appears to be between $-10 \%$ and $+10 \%$ for a $\varnothing=40 \mathrm{~mm}$ sample. This additional lack of uniformity can be, in part, attributed to the windings positioned at a smaller radius than modelled and a slightly larger sample diameter being analysed. Looking at the histogram of gradient uniformity over the entire sample volume, a large majority of the sample experiences a gradient within $5 \%$ of the central gradient, with less than $15 \%$ of the sample volume experiencing a gradient which deviates from the central gradient strength by more than $5 \%$. Upon visual inspection, the windings do not appear to have more variation in position when compared to the windings of the $y$-gradient coil. 
This large range of uniformity is less than desirable, but because the full uniformity of the coil is not necessary for the imaging methods for which it is intended, it should suit the experimental purposes. Once potted, this coil may be used to acquire images such that the actual effect on the imaging components of the proposed techniques can be determined, and assess whether or not this mapping is truly representative of the magnetic field the coil produces.

Before pulsing high currents through the coils in this gradient stack, precautions must be taken to prevent overheating and mechanical failure. Due to the $\mathbf{B}_{\mathbf{0}}$ field experienced by the gradient stack, the wires in each coil will experience Lorentz forces when current is pulsed through them, and will also experience heating due to resistive power dissipation. Both thermal and mechanical stability are accomplished by potting the windings in a thermally conductive epoxy resin.

The potting resin used for this coil is ER-2183 (Electrolube). The thermal conductivity of this resin resembles that of a plastic, with a value of $1.1 \mathrm{~W} / \mathrm{mK}$. It is an electrical insulator possessing a volume resistivity of $10^{15} \Omega \mathrm{cm}$ and dielectric strength of $10 \mathrm{kV} / \mathrm{mm}$, and very hard with a tensile strength and compressive strength of $60 \mathrm{MPa}$ and $120 \mathrm{MPa}$ respectively. The operational temperature is rated from -40 to $130{ }^{\circ} \mathrm{C}$.

After potting the gradient stack using a mould, it was mounted in the $2 \mathrm{MHz}$ system. A 2-D phase encoded image was obtained using this gradient stack to further characterise the fields produced by the coils. A plastic square prism container, $26.8 \mathrm{~mm}$ wide, 50 $\mathrm{mm}$ tall, filled with copper sulfate doped water, was imaged using the $x$ and $y$ gradient coils. With a small DC current running through the second order coil, the 2-D phase encoded image yields an echo for each pixel in space, whose phase evolution in time gives the corresponding field strength at that position due to the second order field coil. This is similar to chemical shift imaging [113] and NMR field mapping [114] where a spectrum is obtained for each pixel. The image in Fig. 6.13 shows the field strength of the second order coil in the $x y$-plane averaged over the $z$-direction. The uniformity of the $x$-gradient degrades at the edge of the sample and thus distorts the image. Despite this lack of uniformity in the $x$-gradient at the edge of the sample, all fields produced by this gradient stack are more than adequate for the proposed experiments, with the values for $C$ obtained by this image and the 3 -axis probe agreeing well where no image distortion 


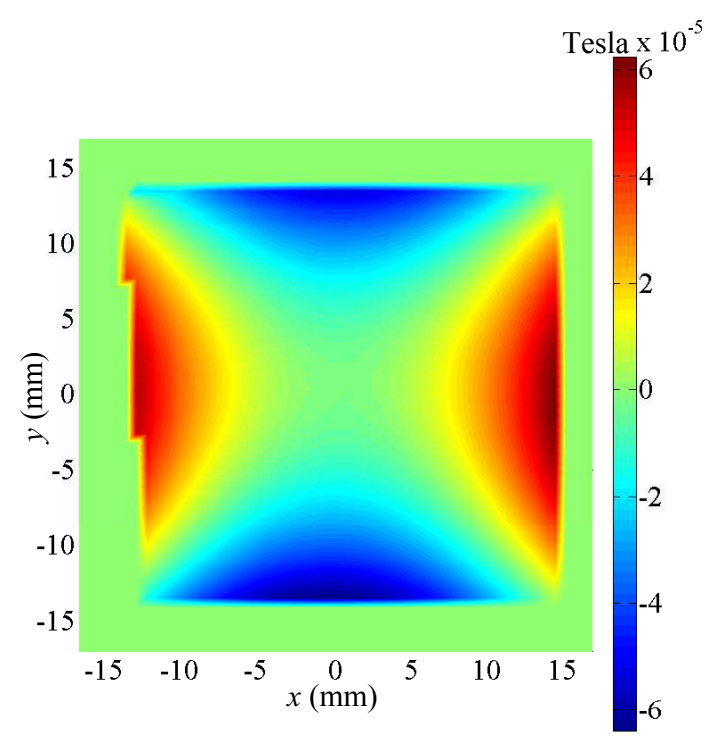

Figure 6.13: 2D phase encoded image of a $26.8 \mathrm{~mm}$ wide $50 \mathrm{~mm}$ tall square prism sample. The intensity of the image shown on the colour scale represents the field strength due to the second order magnetic field coil.

is observed.

\subsection{Coil Design for Superconducting Geometry}

\subsubsection{Design Restrictions}

The design process for the second order field coil for experiments in the $1.5 \mathrm{~T}$ magnet with superconducting geometry are analogous to that for the process discussed for the Halbach geometry. For this reason, this author spares the reader the detailed analysis presented for the Halbach geometry, with the understanding the same processes can be followed for this case. For superconducting geometry, the desired second order magnetic field is $B_{z}=\frac{C}{2}\left(x^{2}-z^{2}\right)$. This field is generated by using the same design as for the second order coil for Halbach geometry, with the coil rotated $90^{\circ}$ about the $x$ axis such that the $y$ component of the magnetic field becomes the $z$ component. This is convenient in the sense that the same design is used, but now different spatial restrictions are imposed due 
to the coil former no longer being concentric with the NMR magnet bore. For maximum
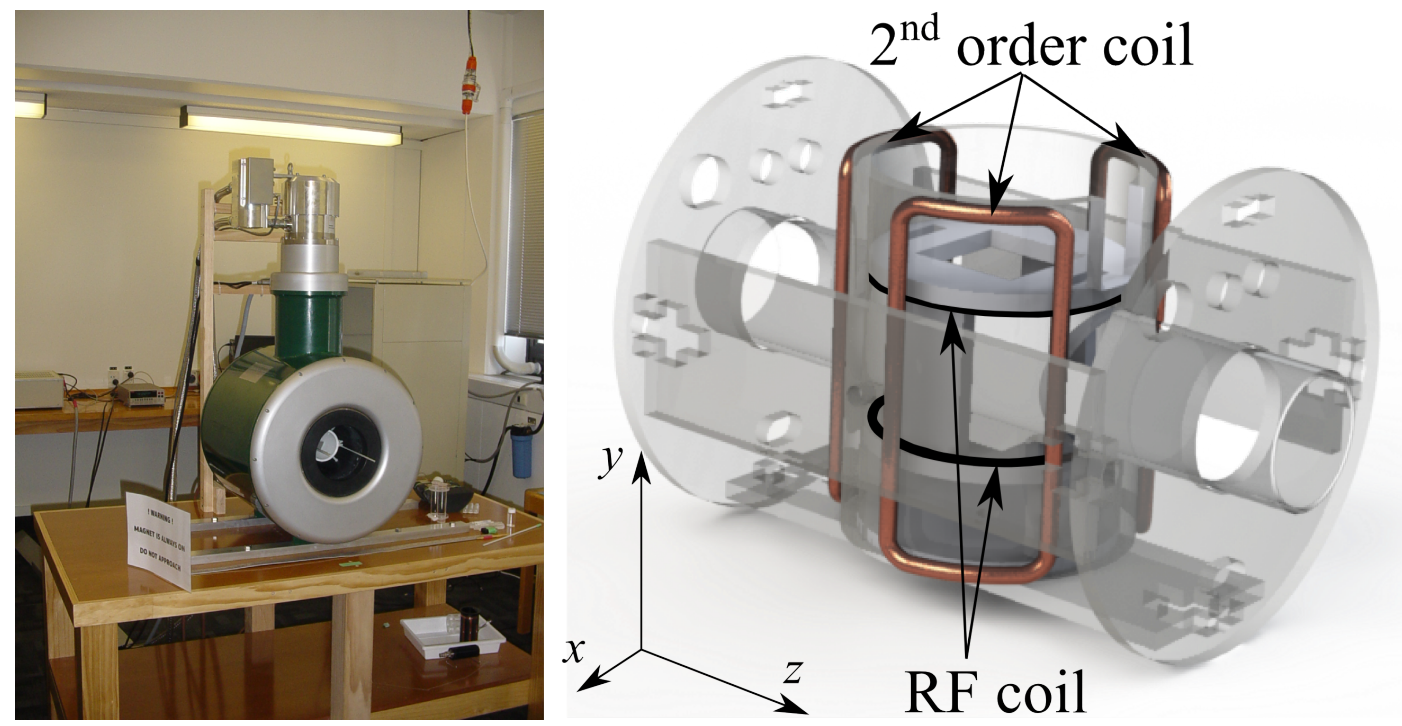

Figure 6.14: Left: $1.5 \mathrm{~T}$ cryogen free superconducting magnet from Cryogenics. Right: Second order and RF coil insert for the superconducting system shown to the left.

uniformity of the field curvature $C$, a large coil diameter and length are desirable. Since the gradient former and magnet bore are no longer concentric, the second order field coil cannot be lengthened without reducing its diameter, and vice versa. The spatial restraint for this design is the magnet bore diameter of $80 \mathrm{~mm}$. An optimal coil diameter of 50 $\mathrm{mm}$ and length of $60 \mathrm{~mm}$ was found which produced a uniformity of $C$ better than $5 \%$ over a $20 \times 20 \times 20 \mathrm{~mm}$ sample volume.

A total of ten turns per loop of $0.475 \mathrm{~mm}$ diameter solid core copper wire was chosen for winding. This results in a resistance of $0.4 \Omega$ which is too low for the amplifier to drive in a controlled manner, and a $1 \Omega$ resistor was placed in series outside of the system to increase the resistance. A rating of $C=1.35 \mathrm{~T} / \mathrm{m}^{2} \mathrm{~A}$ was determined through modelling, and produces the desired diffusive attenuation at the edge of the sample when driven by a B-AFPA 40 amplifier using the experimental parameters considered in subsection 6.3.3 for the low field Halbach magnet. 


\subsubsection{Fabrication}

The carriage and coil former for the second order and RF coils are all composed of perspex. The carriage was laser cut from perspex sheets, and the former cut from a tube of perspex. To wind the second order field coil, holes were drilled and tapped, and nylon screws inserted to form the vertices for the coil windings. The wire was wound onto the former, glued to itself and the former surface, and the screws removed as to not impede its insertion into the carriage and the NMR system.

To allow for sample insertion and removal for the superconducting geometry hardware, holes were drilled through the centre of the second order field coil former. A Helmholtz $\mathrm{RF}$ coil consisting of two loops of wire separated by $23 \mathrm{~mm}$ was then wound on the inner surface of the gradient former with its turns directly above and below the holes. This $\mathrm{RF}$ geometry produces a uniform field in the $x$-axis over the desired sample volume, and still allows for insertion and removal of samples.

It was found that a strong coupling existed between the second order field coil and the RF coil due to their close proximity. When the RF coil was pulsed, there was a significant DC response from the amplifier driving the second order coil of up to $8 \mathrm{~A}$. For this reason, the $\mathrm{RF}$ coil was removed, and a thin copper shield placed on the inner surface of the second order coil former. The RF coil was then insulated from the shield and wound back to its previous location. With the RF shield grounded, this reduced the DC response of the amplifier driving the second order coil by more than two orders of magnitude when the RF coil was pulsed. Without this shield in place, experiments were nearly impossible to perform.

\subsection{Conclusions}

This chapter has presented the design, fabrication, and characterisation for hardware able to produce gradients strengths strong and uniform enough to perform the new experimental techniques presented in chapter 5 . With the well defined hardware built to the desired specifications, the next chapter presents the experimental implementation of the new measurement techniques, along with their limitations and experimental results. 


\section{Chapter 7}

\section{Experimental Results}

\subsection{Introduction}

In this chapter, the experimental methods proposed in chapter 5 are implemented with the custom hardware designed and built in chapter 6 to provide a proof of concept for well defined samples and systems. By using systems which are well understood theoretically, the experimental results in this chapter are compared against theoretical predictions to validate the accuracy of these measurement techniques, and their viability for characterising unknown systems or processes which could not otherwise be resolved.

\subsection{Low Field Parallel Acquisition of $q$-Space \& Single- Shot Diffusion Measurements}

\subsubsection{Introduction}

In this section the pulse sequence for the parallel acquisition of $q$-space as described in subsection 5.3.1 is implemented on the $2 \mathrm{MHz}$ Halbach geometry NMR system to make single-shot diffusion measurements both with signal averaging using the slice selection, and with a true single scan, single-shot diffusion measurement without slice selection. The average propagator for diffusion is also measured using the parallel acquisition of 
$q$-space using signal averaging. Although some of the results presented in this section rely on signal averaging, they are performed to provide a proof of concept, with the understanding that hyperpolarised fluids or higher field strengths may alleviate the need for signal accumulation.

\subsubsection{Diffusion Measurements with Slice Selection}

The parallel acquisition of $q$-space and single shot diffusion measurement as previously described in subsection 5.3.1 and illustrated in Fig. 5.1, is again shown in Fig. 7.1. This new method was first performed using a $2 \mathrm{MHz}$ Rock Core Analyser (Magritek Ltd.). The parameters used for this new measurement technique were slice selections along the $x$ direction of 2,3 , and $5 \mathrm{~mm}$ on a $26.8 \mathrm{~mm}$ wide, $50 \mathrm{~mm}$ tall square prism distilled water sample. The sample was oriented such that the length of the prism was aligned and centred along the $z$ axis, and its faces were centred and normal to the $x$ and $y$ axes of the linear and constant gradient fields (Fig. 7.2). This sample geometry was chosen

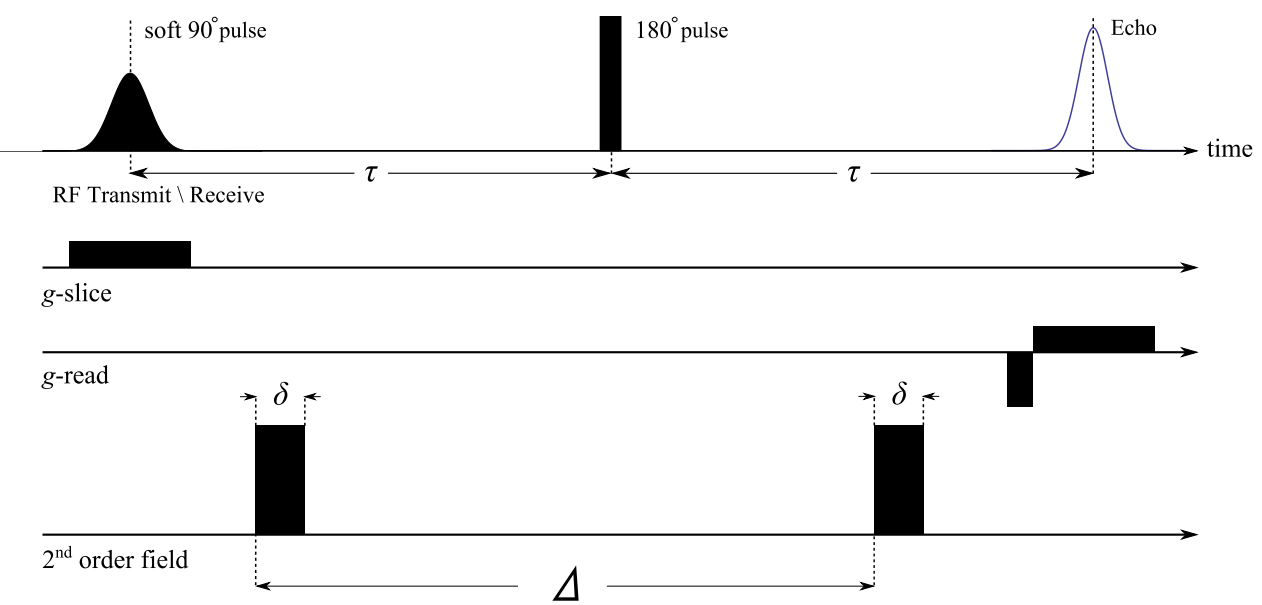

Figure 7.1: Pulse sequence for the parallel acquisition of $q$-space.

such that the image modulation function $A(\rho, d)$ from Eq. 5.11 is constant across the sample for any slice thickness, and a Gaussian fit could be applied directly to the image to obtain a diffusion measurement as described in Eq. 7.1. If the more common cylindrical sample geometry was used, this modulation function would no longer be constant along 


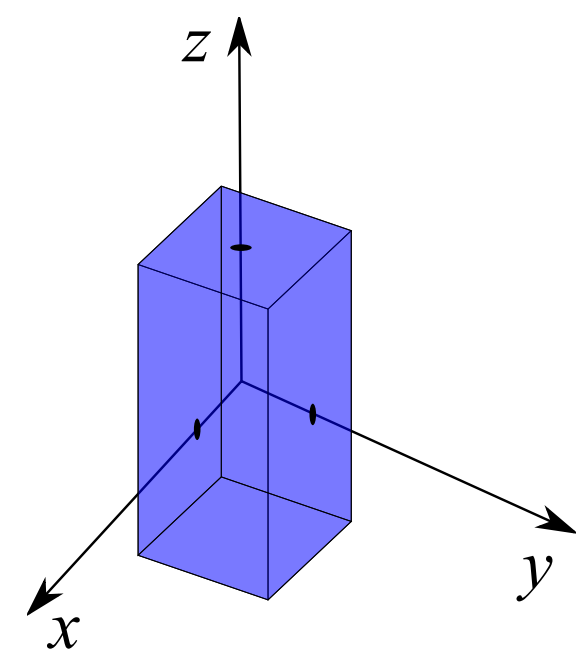

Figure 7.2: Square prism, distilled water sample. The faces of the prism are aligned normal to the $x, y$, and $z$ axes of the linear and constant gradient fields, and the sample is centred at the origin inside the linear and constant gradient coils.

the direction of the read image, and the fitting function applied to the image to obtain the diffusion measurement would be the product of $A(\rho, d)($ Eq. 5.10) and a Gaussian as described in Eq. 5.11. An observation time of $\Delta=30 \mathrm{~ms}$ was used with second order field pulses of $4 \mathrm{~ms}$, and $C=14 \mathrm{Tm}^{-2}$. These parameters were chosen such that the values $\delta, C$, and $\Delta$ result in full diffusive attenuation of the signal at the edge of the sample, $\delta$ is still small compared to $\Delta$, and the amplifier driving the second order field coil is not strained to produce the necessary pulse amplitude. These parameters were chosen such that $T_{2}$ relaxation does not significantly affect the signal amplitude, and eddy currents do not interfere with the measurement. The characteristic length scale of the second order field for these parameters is calculated as $l_{c}=365 \mu \mathrm{m}$, and the diffusion length $d_{l}=12 \mu \mathrm{m}$, making $l_{c} \gg d_{l}$, and the condition in Eq. 5.20 easily holds. This allows the diffusion encoded image to be related to the diffusion coefficient through the relationship given by Eq. 5.23. Since this is such a significant relationship for this chapter, Eq. 5.23 is provided again as

$$
E_{N}(y)=\exp \left\{-(\gamma \delta C y)^{2} D(\Delta-\delta / 3)\right\}
$$


A $y$-read gradient of $4.7 \mathrm{mT} / \mathrm{m}$ was used with 64 points acquired, and a dwell time of $100 \mu$ s per point, yielding 32 pixels across the entire sample. This results in the read image spread out over a bandwidth which is roughly 70 times the spectral linewidth of a Hahn echo, and any line shape effects due to the $B_{0}$ inhomogeneities do not distort the read image. For these parameters, the ratio of the diffusion length to pixel width is $\sqrt{2 D \Delta} / p w=1.4 \times 10^{-2}$, and any effects arising from diffusion between pixels can also be ignored. Since these experiments were performed on a $2 \mathrm{MHz}$ system, signal to noise was insufficient for such thin slice volumes, and 32 scans were used strictly for signal averaging without phase cycling, adhering to the single-shot approach.

The sequence was run first without the second order field pulses to collect a one dimensional image of the slice, and again with the second order field pulses such that the diffusion information was encoded into the image. Here it is noted that the reference image only demonstrates the imaging portion of the pulse sequence is working as expected, and plays no part in the acquisition of $q$-space data or the diffusion measurement. In Fig. 7.3, the reference images are shown as solid curves and the diffusion encoded image plotted as data points, both normalised with respect to the mean reference image amplitude. By mapping position onto $q$-space through the relationship $q=-\gamma \delta C y$, a Gaussian curve is fit to the image to extract the diffusion coefficient.

The distilled water sample at a temperature of $27^{\circ} \mathrm{C}$ has a known diffusion coefficient [115] of $D=2.42 \times 10^{-9} \mathrm{~m}^{2} / \mathrm{s}$, and it was found that all images, under a Gaussian fit, agree well with this value. These data sets are also presented in the more familiar StejskalTanner plot shown in the bottom right of Fig. 7.3, with each data set normalised with respect itself, and the theoretical signal attenuation shown in dashed red.

If the field experienced by a selected slice from the second order field pulses is well approximated by a parabola, the diffusion encoded image normalised with respect to the reference image should obey the relationship in Eq. 7.1. In the $2 \mathrm{~mm}$ slice experiment, no noticeable signal attenuation of the diffusion encoded image is observed at $y=0$ with respect to the reference image. This is a good indicator that the gradient strength at $y=0$ is $g(y=0) \approx 0$, the parabolic field approximation should hold, and a well defined mapping between real space and $q$-space exists. For the $3 \mathrm{~mm}$ slice, a very slight attenuation around $y=0$ was observed as the parabolic field approximation is less accurate. For the 

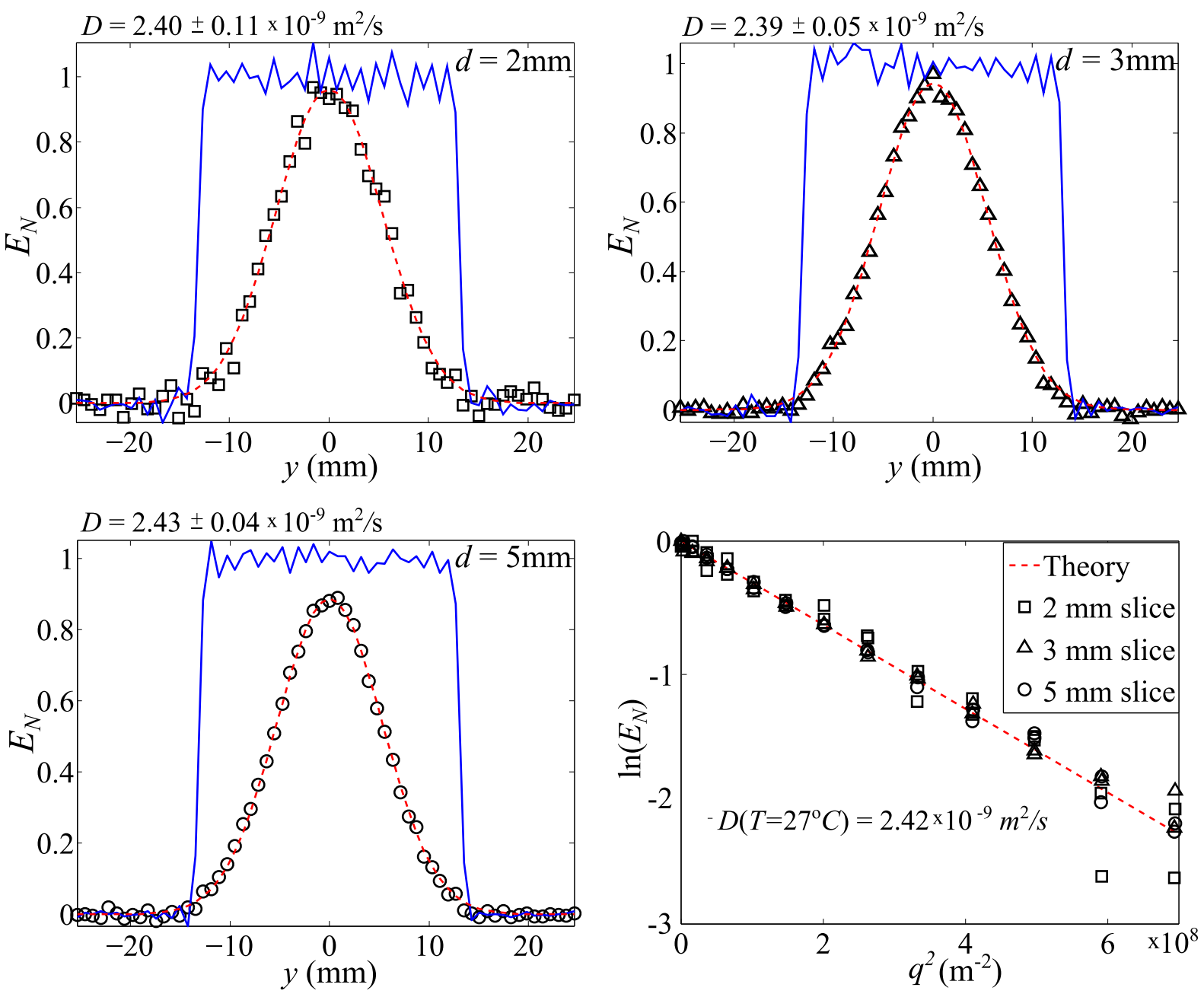

Figure 7.3: Reference and diffusion encoded images for varying slice thickness $d$ at an observation time of $\Delta=30 \mathrm{~ms}$. Solid curves: reference images. Points: Diffusion encoded images. Dashed curves: Gaussian fits to diffusion encoded images. Bottom right: Stejskal-Tanner plot of diffusion data sets as points, and theoretical plot as a dashed curve. 
$5 \mathrm{~mm}$ slice, it becomes apparent that the parabolic field approximation is not accurate as the diffusion encoded image is noticeably attenuated at $y=0$, showing the thickness of the slice has sampled significant non-zero gradient values, and the mapping between real space and $q$-space is no longer well defined. Here it is noted that as predicted from the previous analysis in subsection 5.3.2, the diffusion measurement is immune to the range of gradient strengths across the thickness of the slice, and the only effects of increasing slice thickness are an increase in signal to noise, and increased overall attenuation of the diffusion encoded image with respect to the reference image.

\subsubsection{Echo as the Diffusion Propagator}

With no apparent attenuation of the diffusion encoded image with respect to the reference image at the origin, the parabolic field approximation is accurate. The diffusion encoded image may then be interpreted as $E(\mathbf{q})$, and its Fourier transform is the average propagator $\bar{P}(\mathbf{R}, \Delta)$, making the echo the average propagator $E(t) \propto \bar{P}(\mathbf{R}, \Delta)$ as discussed in section 5.4 .

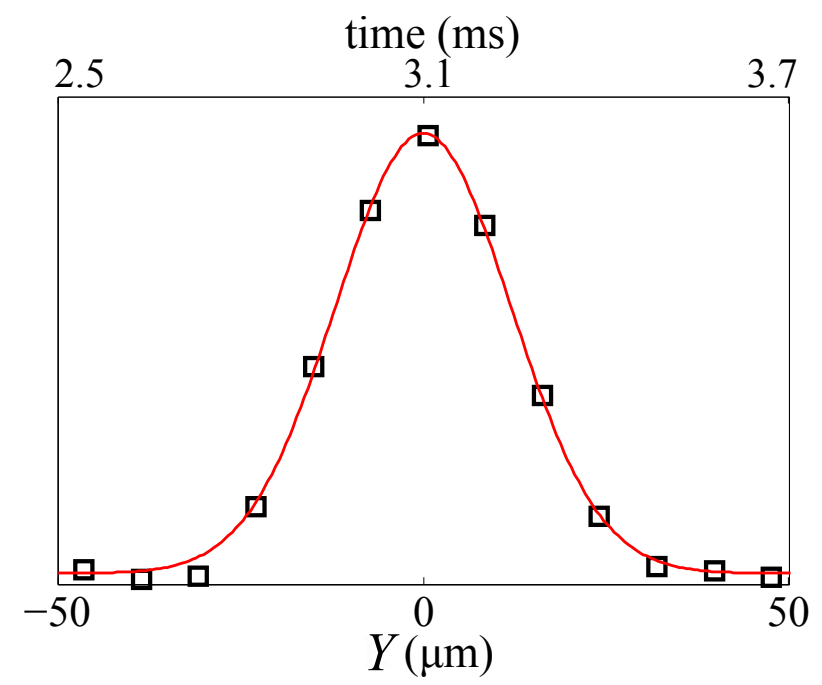

Figure 7.4: Truncated echo data plotted as points versus displacement space and time. The solid curve represents the theoretical propagator for free diffusion for the observation time $\Delta=30 \mathrm{~ms}$, calculated using the measured diffusion coefficient from the diffusion encoded image for the $2 \mathrm{~mm}$ slice experiment shown in Fig. 7.3. 
Since it was previously determined in subsection 7.2.1 that the parabolic field approximation for the $2 \mathrm{~mm}$ slice experiment holds, and therefore a well defined mapping between real space and $q$-space exists, the echo for this experiment can be mapped onto displacement space. Setting the acquisition time equal to the FOD defined in subsection 5.4.3, and normalising the echo signal such that its integral is unity, the average propagator is obtained (Fig. 7.4). In this case displacement space, or $\mathbf{R}$, is taken as the displacement along the $y$-axis such that $R=Y$. The solid curve represents the theoretical propagator calculated using the measured diffusion coefficient from the diffusion encoded image, and the Gaussian propagator for free diffusion given by Eq. 2.11.

\subsubsection{Diffusion Measurements Without Slice Selection}

As the slice thickness increases, a larger range of gradients is sampled along the slice direction for each pixel in the diffusion encoded image. This results in the loss of a meaningful real space to $q$-space mapping, with an increase in signal to noise, while retaining the ability to measure the diffusion coefficient. In this section the proposed single-shot diffusion measurement was employed in a true single-shot fashion to measure the diffusion coefficient in a single scan at low field for multiple second order field pulse amplitudes $C$. As with conventional PGSE experiments, increasing the range of diffusive signal attenuation over which data is acquired will help to reduce error in the diffusion measurement. In the slice selective experiments, parameters were chosen such that attenuation down to noise level was achieved at the edge of the sample in the read direction, ensuring a reliable measurement of $D$. Here, the pulse amplitude of the second order field is varied from experiment to experiment to assess the accuracy of the diffusion measurement over different ranges of diffusive attenuation.

In the left panel of Fig. 7.5, each image was acquired using a single scan, with the second order field pulse amplitude varied from experiment to experiment. The corresponding diffusion coefficients for each scan are plotted in the right panel of Fig. 7.5 against the amount of diffusive attenuation experienced at the edge of the sample with respect to the signal amplitude at the centre of the sample. The experimental parameters used were $\Delta=30 \mathrm{~ms}, \delta=4 \mathrm{~ms}$, with a $y$-read gradient of $4.7 \mathrm{mT} / \mathrm{m}$, acquiring 128 points with a 

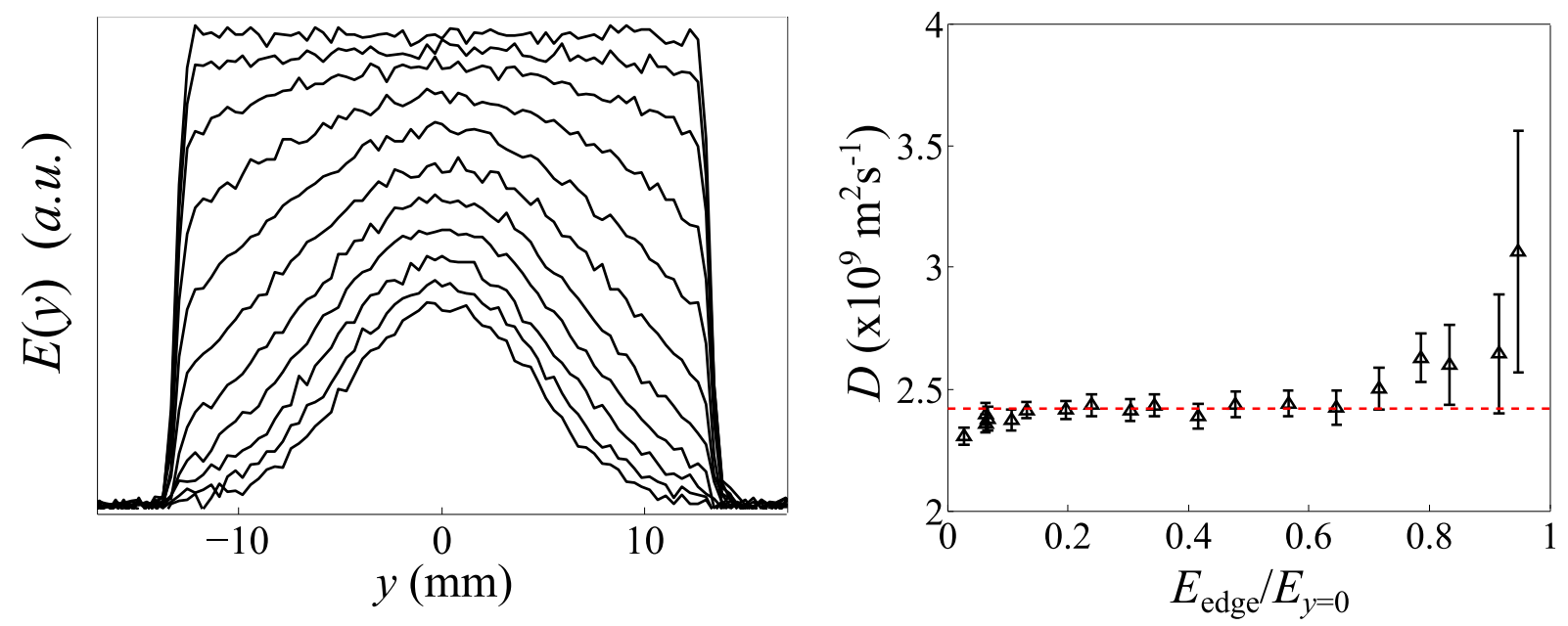

Figure 7.5: Left: Single-scan diffusion encoded images for varying second order field pulse amplitudes, with pulse amplitude defined by the curvature of the second order field $(C)$. Overall attenuation is increased for increased pulse amplitude. Each scan yields an image. Each image yields a diffusion measurement. Only a subset of the images are shown to avoid crowding the plot. Right: Single-scan / single-shot diffusion measurements for varying second order magnetic field pulse amplitudes $(C)$. Each diffusion measurement corresponds to a single image acquired in a single scan.

dwell time of $100 \mu$ s per point, yielding 64 pixels across the entire sample. In contrast to the slice selective experiments, the entire sample is now excited and a higher signal to noise is available. Since the signal to noise of each pixel is equal to the entire signal divided by the number of pixels in the image, the number of pixels in these imaged are increased with respect to the slice selective experiments, while retaining sufficient signal to noise in each pixel for a reliable diffusion measurement.

As the amplitude of the second order field pulse was increased, an increase in overall image attenuation was observed (left panel Fig. 7.5), accompanied by a decrease in measurement error (right panel Fig. 7.5) which appears to reach a minimum. This effect is due to the overall diffusive attenuation of the image for a thick slice. This overall attenuation, combined with the noise level for a single scan, limits the relative attenuation at the edge of the image with respect to the center, and therefore limits the accuracy of the measurement. 
The relative attenuation of the diffusion encoded image is the ratio of the signal amplitude at the edge of the image

$$
E_{y=e d g e}=A(\rho, d) \exp \left\{-\left(\gamma \delta C y_{\text {edge }}\right)^{2} D(\Delta-\delta / 3)\right\}
$$

to the signal amplitude at the origin

$$
E_{y=0}=A(\rho, d)
$$

where

$$
A(\rho, d)=\int_{-d / 2}^{d / 2} \rho(\mathbf{r}) \exp \left\{-(\gamma \delta C x)^{2} D(\Delta-\delta / 3)\right\} d x .
$$

Taking the ratio of $E_{y=e d g e}$ to $E_{y=0}$, using Eq. 7.2 and Eq. 7.3, the relative range of attenuation may be expressed as

$$
\frac{E_{y=e d g e}}{E_{y=0}}=\frac{A(\rho, d) \exp \left\{-\left(\gamma \delta C y_{\text {edge }}\right)^{2} D(\Delta-\delta / 3)\right\}}{A(\rho, d)} .
$$

In the case of infinite signal to noise, the terms $A(\rho, d)$ in the numerator and denominator will cancel and the relative range of attenuation can be expressed as

$$
\frac{E_{y=e d g e}}{E_{y=0}}=\exp \left\{-\left(\gamma \delta C y_{\text {edge }}\right)^{2} D(\Delta-\delta / 3)\right\}
$$

In the actual case where signal to noise is limited, noise level considerations prevent the term $A(\rho, d)$ in the numerator and denominator of Eq. 7.5 from cancelling. As $C$ is increased, the expression for $E_{y=e d g e}$ in Eq. 7.2 will reach its minimum as the signal becomes lost in the noise. Once the value of $C$ is increased past this point, the expression for $E_{y=0}$ in Eq. 7.3 will continue to decrease towards the noise level while $E_{y=e d g e}$ remains constant at the noise level. Therefore, increasing $C$ above the amplitude necessary to attenuate the signal at the edge of the image into the noise will begin to yield a lesser range of relative attenuation across the image. To increase the maximum range of relative attenuation, an increase in signal to noise is necessary, either through signal averaging or a stronger $B_{0}$ field. 


\subsection{Low Field Diffusion Measurements Using the Mod- ified Difftrain Pulse Sequence}

\subsubsection{Introduction}

In this section the modified Difftrain pulse sequence described in subsection 5.3.4 is used to make diffusion measurements at multiple observation times in a single experiment using signal averaging in the $2 \mathrm{MHz}$ Halbach geometry NMR system, providing the surface to volume ratio and tortuosity of well characterised porous media as described in subsection 2.4.2. Although multiple scans are required to obtain sufficient signal to noise to performed the desired measurements, the results provide a proof of concept for the technique with the understanding that hyperpolarised fluids or higher field strengths may alleviate the need for signal accumulation.

\subsubsection{Samples}

Three different samples were used for experimental purposes in this section, which are characterised in this subsection for further reference. The samples used are labelled sample A, B, and C and are defined as follows.

Sample A consists of a square prism $26.8 \mathrm{~mm}$ wide, $50 \mathrm{~mm}$ tall, filled with distilled water. Sample B consists of a square prism $26.8 \mathrm{~mm}$ wide, $50 \mathrm{~mm}$ tall, filled with 125-150 $\mu \mathrm{m}$ diameter polyethylene beads (Cospheric), with the pore space saturated with methanol. A CPMG sequence [73] was used to acquire an echo train, to which an exponential was fit to the amplitude of the echo train, and the signal amplitude extrapolated at the initial point of spin excitation. This process was also performed for a volume of methanol equal to the total volume of the saturated bead pack. The ratio of the signal amplitudes gives the ratio of methanol present in the pore space to the entire sample volume, and the porosity of the bead pack. The porosity was calculated as $\phi=0.36 \pm 0.01$. For a monodisperse bead pack, the surface to volume ratio is given by

$$
S / V=6(1-\phi) / \phi b
$$


where $b$ is the bead diameter[14]. An estimate can be made for the surface to volume ratio of this bead pack using Eq. 7.7 and the bead diameter and porosity ranges. This method predicts the surface to volume ratio of sample B as

$$
6.8 \times 10^{4} \mathrm{~m}^{-1} \leq S / V \leq 8.9 \times 10^{4} \mathrm{~m}^{-1}
$$

The tortuosity $\alpha$ of this bead pack can be estimated [116] using the approximation $1 / \alpha \approx \sqrt{\phi}$. Using the measured porosity, this predicts $0.59 \leq 1 / \alpha \leq 0.61$.

Sample C consists of a square prism $26.8 \mathrm{~mm}$ wide, $50 \mathrm{~mm}$ tall, filled with $215-250 \mu \mathrm{m}$ diameter polyethylene beads (Cospheric), with the pore space saturated with methanol. The same method was used to estimate the porosity of the sample, and was measured as $\phi=0.36 \pm 0.01$. This predicts the surface to volume of sample $\mathrm{C}$ as

$$
4.1 \times 10^{4} \mathrm{~m}^{-1} \leq S / V \leq 5.2 \times 10^{4} \mathrm{~m}^{-1}
$$

\subsubsection{Free Diffusion Measurements}

The modified Difftrain pulse sequence described in subsection 5.3.4 and previously shown in Fig. 5.4 is again illustrated in Fig. 7.6. This pulse sequence was run using sample A to measure the free diffusion of water. Experimental parameters were set to observation times at multiples of $30 \mathrm{~ms}, \delta=4 \mathrm{~ms}, C=6 \mathrm{Tm}^{-2}$, a $y$-read gradient of $4.7 \mathrm{mT} / \mathrm{m}$, 38 points taken across the sample, a $100 \mu$ s dwell time, with 10 stimulated echoes in the pulse sequence. These experimental parameters give a maximum diffusion length of $d_{l}=36 \mu \mathrm{m}$, and a characteristic length scale of $l_{c}=558 \mu \mathrm{m}$ such that $l_{c} \gg d_{l}$, and Eq. 7.1 holds. Due to the large number of echoes and limited signal to noise at such a low field, 64 scans were used to signal average without phase cycling.

The recall RF pulse amplitudes were varied such that the reference images shown as dashed lines in the left plot of Fig. 7.7 were of slightly more equal amplitude than for a constant recall tip angle. It is noted that the reference images play no role in the diffusion measurement, and are provided only to illustrate that the imaging portion of the pulse sequence is functioning as expected. Since the first second order field pulse sets the range of $q$ values which will be sampled for each image at all observation times, 


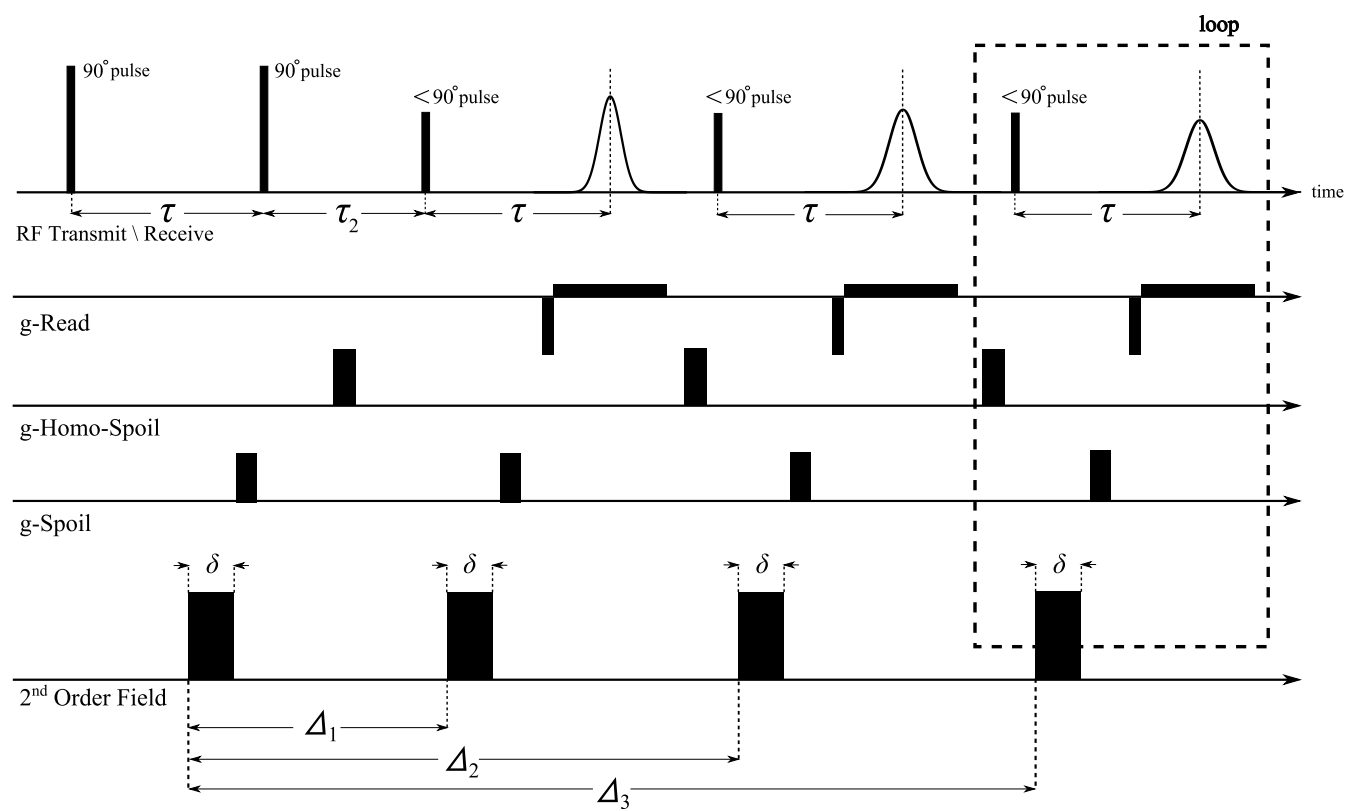

Figure 7.6: The modified DiffTrain pulse sequence including components enabling measurement of the apparent diffusion coefficient at multiple encoding times.

there is a balance between obtaining enough signal attenuation at the short observation times for an accurate diffusion measurement while not fully attenuating the signal at the longest observation time. Since the range of observation times is large, a fairly small range of $q$ is chosen with the first second order field pulse such that signal is not completely attenuated for the largest observation time. This means the attenuation across the image for the smallest observation time is small, and more signal to noise is necessary to obtain an accurate diffusion measurement. Therefore, the images obtained at shorter observation times are left with larger amplitude than those at longer observation times. With this scheme, the error observed in the diffusion measurements when fitting a Gaussian to each diffusion encoded image remains relatively stable across all observation times. As expected, since there is no restriction experienced by the diffusing molecules, the measured diffusion coefficient remains constant independent of observation time (right Fig. 7.7), and agrees well with the known value[115] of $2.22 \times 10^{-9} \mathrm{~m}^{2} \mathrm{~s}^{-1}$ at $23.5^{\circ} \mathrm{C}$ for all measurements. This experiment shows that the pulse sequence is working as expected, and is able to measure the apparent diffusion coefficient at multiple observation times in 

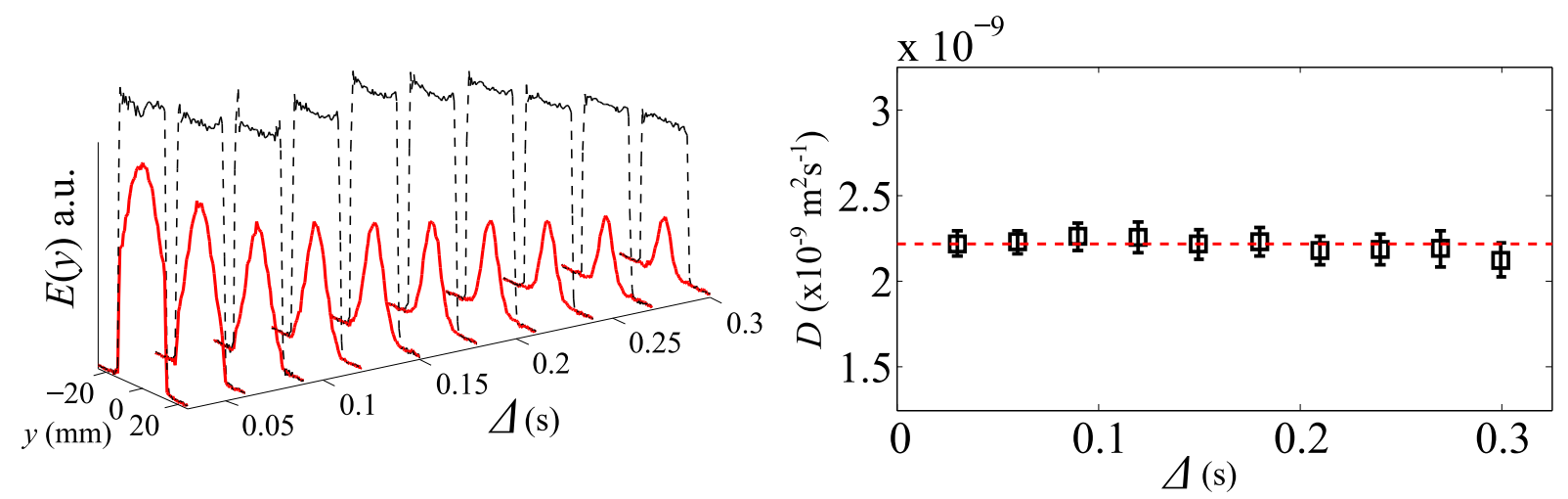

Figure 7.7: Free diffusion measurements of bulk water using the modified Difftrain pulse sequence from Fig. 7.6. Left: Dashed curves represent reference images acquired without the second order field pulses, and solid curves represent the diffusion encoded images acquired with the second order field pulses. Right: Diffusion measurements made from curve fitting to the diffusion encoded images. The dashed line represents the known value for free diffusion of water at $23.5^{\circ} \mathrm{C}$. As expected, the measured diffusion coefficients are independent of observation time.

a single experiment given enough signal to noise is present.

\subsubsection{Restricted Diffusion Measurements Spanning the Short and Long Time Limits}

For the single-shot surface to volume and tortuosity measurement at $2 \mathrm{MHz}$, the pulse sequence in Fig. 7.6 was run using sample B. With low signal to noise due to a measured porosity of $\phi=0.36$, and low $B_{0}$ field strength, only 20 points were taken across the sample to increase the signal to noise per pixel, and 128 scans used without phase cycling, strictly for signal averaging. This field strength is commonly used in commercial instrumentation for the characterisation of porous media because it reduces the impact of internal magnetic field gradients. Therefore, a proof of concept is presented for surface to volume ratio and tortuosity measurements in this and the following subsection using signal accumulation, with the understanding that hyperpolarised fluids or higher field strength may alleviate the need arising from a low signal to noise ratio at $2 \mathrm{MHz}$ for signal averaging. 

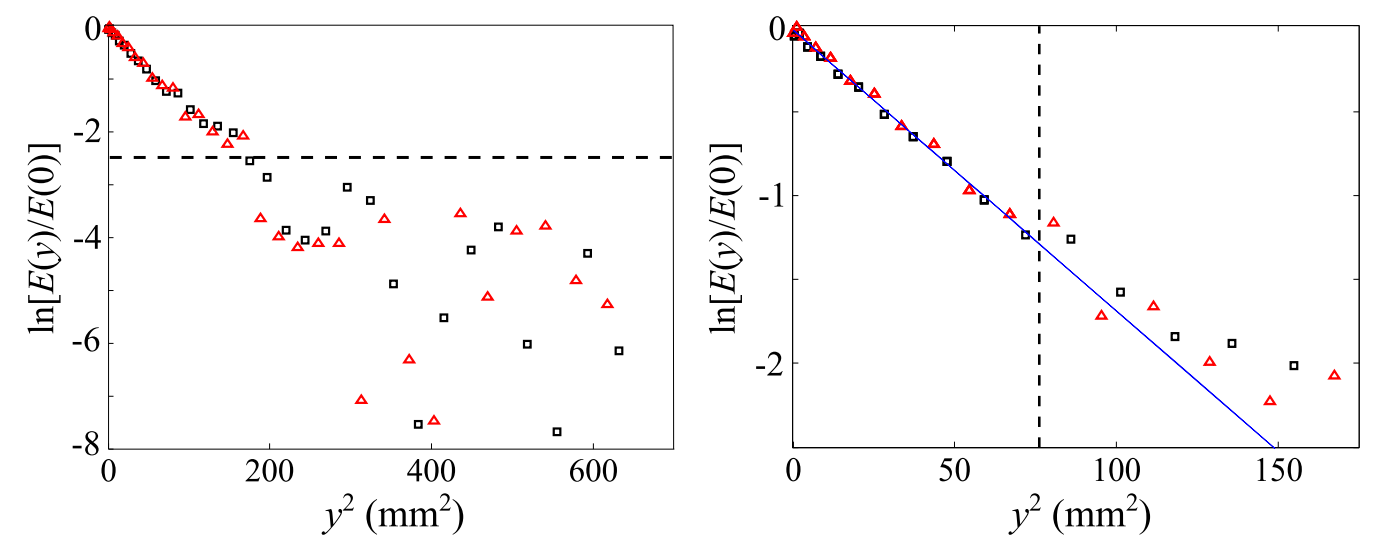

Figure 7.8: Example of data analysis for restricted diffusion. Left: The natural logarithm of the data set from the diffusion encoded image at $\Delta=210 \mathrm{~ms}$ is plotted against the square of position along the $y$ direction. The triangles represent the data points for negative $y$ values, and squares for positive $y$ values. Right: The data set has been truncated to remove data at or below the noise level. The linear fit and dashed vertical line show where the data no longer obeys the linear trend and relationship in Eq. 7.10.

When fitting a Gaussian curve to the diffusion encoded images to obtain the apparent diffusion coefficient, care must be taken to only include the data points which obey the Gaussian relationship between the signal amplitude and position along the read direction from Eq. 7.1. This is most easily done by plotting the natural logarithm of the image data against the square of the position in the read direction. Taking the natural log of Eq. 7.1, the equation describing this linear trend is obtained as

$$
\ln \left(\frac{E(y)}{E(0)}\right)=-(\gamma \delta C y)^{2} D(\Delta-\delta / 3)
$$

An example of the data analysis is shown in Fig. 7.8, where the normalised data from the $7^{\text {th }}$ stimulated echo image is processed. In the left plot of Fig. 7.8, the natural log of the data is plotted against against the squared position along the read direction, with data points corresponding to negative $y$ values plotted as squares, and positive $y$ values as triangles. The horizontal dashed line represents the noise level where the data set is first truncated.

The right plot in Fig. 7.8 shows the data after truncation using the signal to noise 
threshold. The vertical dashed line in the right plot of Fig. 7.8 represents where the data was further truncated as it no longer follows the linear trend given by Eq. 7.10.

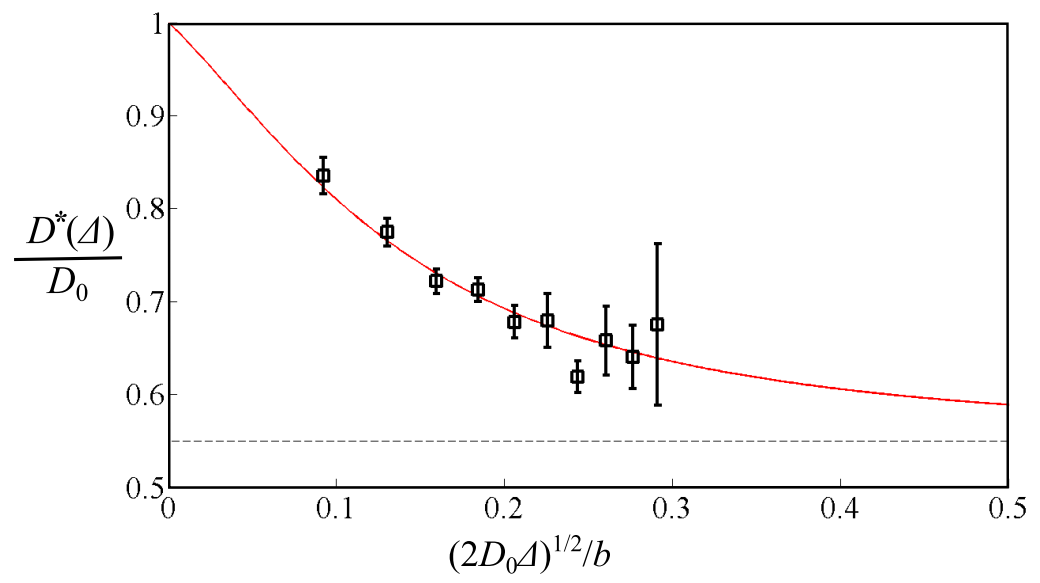

Figure 7.9: The apparent diffusion coefficient is measured at multiple observation times in a single-shot experiment, and is plotted against the diffusion length normalised by the mean bead diameter $b$. The measurements are shown as data points and a two point Padé approximation fit to the data as a solid curve. The slope of the curve in the short time limit reveals the surface to volume ratio of the bead pack. The asymptotic value shown by the dashed line gives the inverse of the tortuosity of the bead pack.

The apparent diffusion coefficients were normalised with respect to the free diffusion coefficient and plotted against a diffusion length normalised by the mean bead diameter in Fig. 7.9. The slope of this data in the short time limit yields a surface to volume ratio [13] of $7.2 \pm 0.5 \times 10^{4} \mathrm{~m}^{-1}$, which agrees well with the predicted value. A two point Padé approximation was fit to the data to estimate the tortuosity in the long time limit. This estimated a value of $1 / \alpha=0.56 \pm 0.05$, and is also in good agreement with the predicted value.

The observation times for this experiment do not sufficiently probe the short time limit where $D_{\text {app }}(\Delta) / D_{0}>0.8$, and rely on an approximation to measure the surface to volume ratio. As previously shown by Latour et al.[14], this is a threshold marking the point where the short time limit approximation resulting in $D(\Delta) \propto \sqrt{\Delta}$ becomes less valid, and higher order terms of $\Delta$ begin to significantly contribute. To provide a more reliable measurement of the surface to volume, observation times were decreased and the number 
of echoes reduced to probe only the short time diffusive behaviour.

\subsubsection{Short Time Limit Restricted Diffusion Measurements}

To measure the surface to volume ratio of sample B in a more robust fashion, the number of stimulated echoes in the pulse sequence illustrated in Fig. 7.6 was reduced to 5, and RF pulse amplitudes adjusted to distribute all stored magnetisation among the 5 echoes. With the signal to noise per scan still too low to reliably perform the surface to volume measurement in a single scan, 64 scans were used without phase cycling to signal average. Experimental parameters were set to $C=8 \mathrm{Tm}^{-2}, \delta=3 \mathrm{~ms}$, observation times at multiples of $18 \mathrm{~ms}$, and the same acquisition and read gradients as before. These parameters give a maximum diffusion length of $d_{l}=20 \mu \mathrm{m}$, and a characteristic length scale of $l_{c}=558 \mu \mathrm{m}$ making $l_{c} \gg d_{l}$, and Eq. 7.1 holds. The images in the left plot of Fig. 7.10 show reference read images as dashed lines at the multiple observation times, and the diffusion encoded images as solid lines. Upon curve fitting to the diffusion encoded images, 5 diffusion measurements are obtained, and shown in the right plot of Fig.
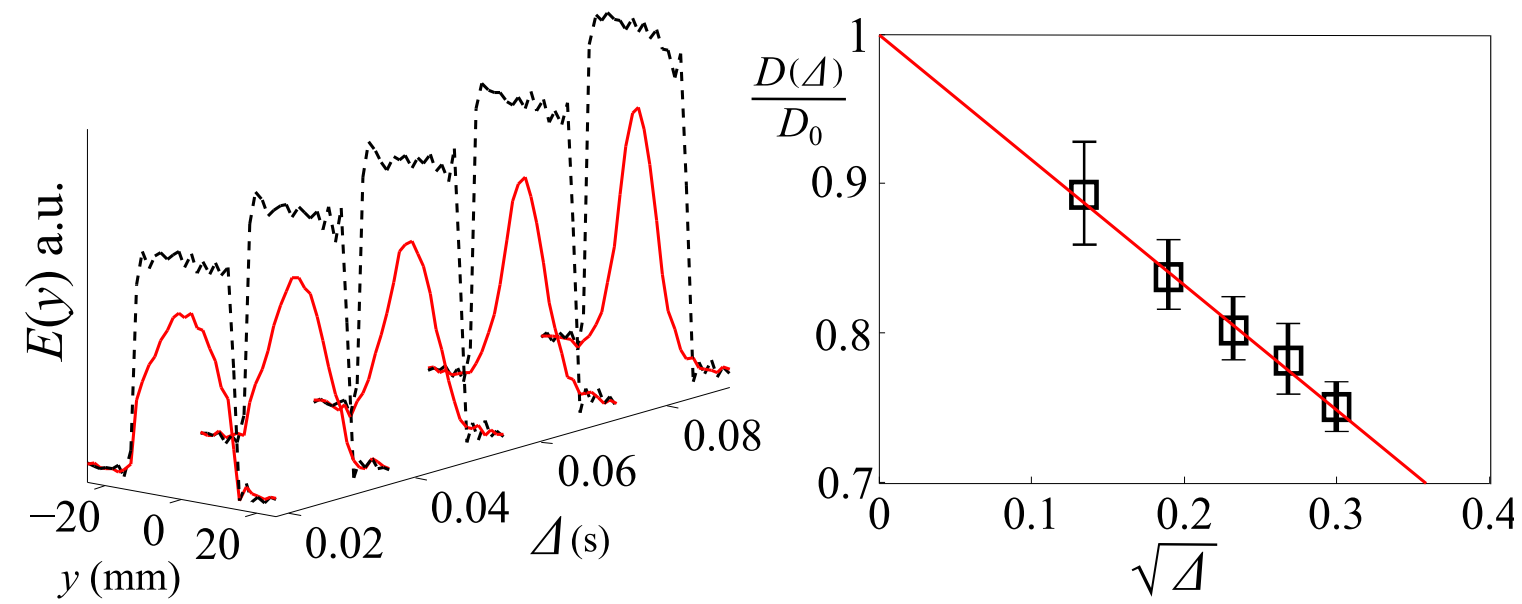

Figure 7.10: Left: Reference images (dashed lines), and diffusion encoded images (solid lines), are acquired at multiple observation times. Right: Diffusion measurements are made by curve fitting to the diffusion encoded images, and are plotted against $\sqrt{\Delta}$. A linear fit is made to the diffusion measurements to extract the surface to volume ratio of the $125-150 \mu \mathrm{m}$ bead pack. 
7.10. The last two diffusion measurements are at or past the point where the short time limit approximation is valid. If the last two diffusion measurements shown in Fig. 7.10 are neglected and the linear fit made only to those data points where $D(\Delta) / D_{0}>0.8$, a value of $7.3 \times 10^{4} \mathrm{~m}^{-1}$ is obtained with an error of $\pm 0.3 \times 10^{4} \mathrm{~m}^{-1}$ when fitting strictly to the data points, and an error of $\pm 2.2 \times 10^{4} \mathrm{~m}^{-1}$ calculated using the error bars for each diffusion measurement, and is in good agreement with the predicted value.

Ideally, shorter observation times would be used for these diffusion measurements to ensure all observation times occur well within the short time limit. These experimental parameters marked the limit where the pulse sequence could be run reliably. When observation times were reduced below $18 \mathrm{~ms}$, spoiler gradient pulses and second order field pulses were in close proximity to RF pulses. Also, a decreased observation time resulted in the need for a shorter and stronger second order field pulses for the desired diffusive attenuation and gradient pulse profiles which were less perfect. As no coils are shielded, further decreasing the observation time resulted in interactions between the coils, and eddy current effects which disrupted the experiment. Since the observation time could not be reduced any further, a bead pack of larger diameter beads was used to modify the relationship between the available observation times and the short time limit approximation. With a larger bead diameter comes larger pore spaces, and therefore the diffusion length can be longer while still remaining small compared to the pore size. This results in a short time limit which is longer in duration, allowing all diffusion measurements to fall within this limit.

To further verify the performance of this method, sample $\mathrm{C}$ was characterised using the same experimental parameters as for the previous 5 echo experiment. The reference images of the sample obtained without the second order field pulses, and diffusion encoded images obtained with the second order field pulses, are shown in the left plot of Fig. 7.11. The diffusion measurements made by curve fitting to the diffusion encoded images are shown in the right plot of Fig. 7.11. With the larger diameter beads and larger pore spaces, the diffusion measurements lie within the short time limit such that $D(\Delta) / D_{0}>0.8$ for all $\Delta$. A linear fit was made to these diffusion measurements to reveal the surface to volume ratio of the bead pack, giving a value of $4.1 \times 10^{4} \mathrm{~m}^{-1}$ with an error of $\pm 0.3 \times 10^{4} \mathrm{~m}^{-1}$ fitting strictly to the data points, and an error of $\pm 1.5 \times 10^{4} \mathrm{~m}^{-1}$ 

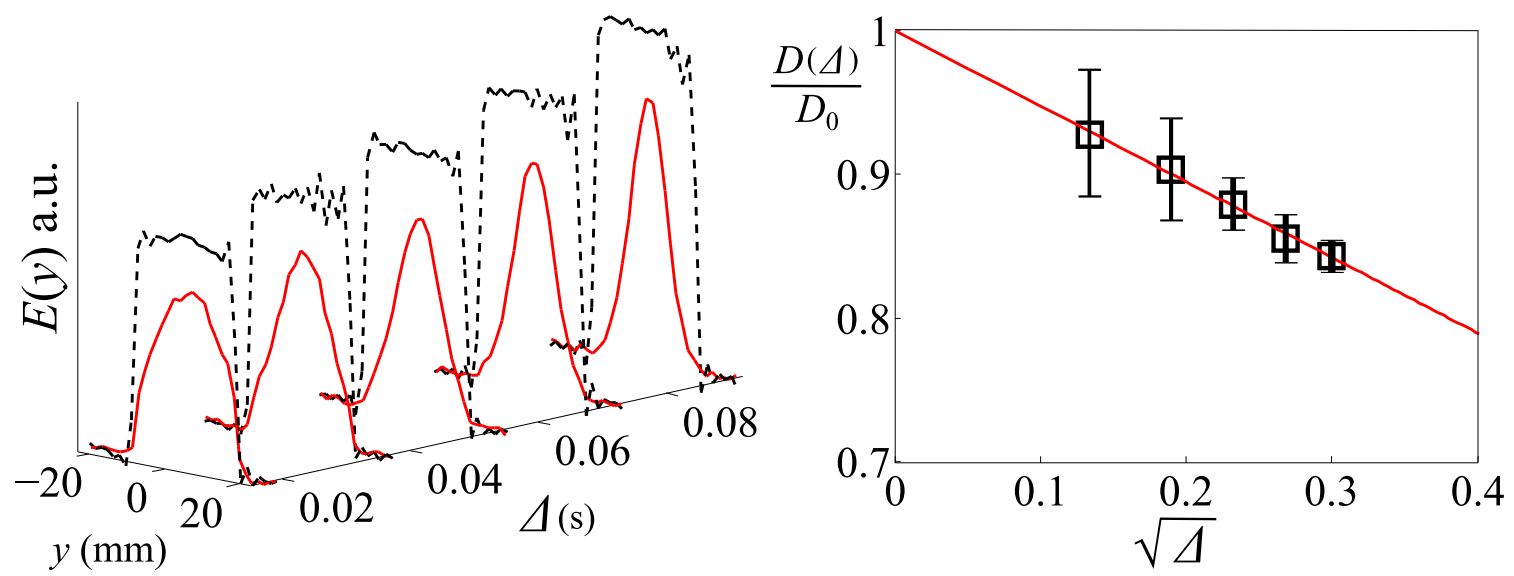

Figure 7.11: Left: Reference images (dashed lines), and diffusion encoded images (solid lines), acquired at multiple observation times. Right: Diffusion measurements are plotted against $\sqrt{\Delta}$. A linear fit is made to the data to extract the surface to volume ratio of the 215-250 $\mu \mathrm{m}$ bead pack.

calculated using the error bars for each diffusion measurement, and is good agreement with the predicted value.

These results provide a proof of concept for the measurement of the apparent diffusion coefficient at multiple times in a single experiment, and a single-shot surface to volume ratio measurement. Although signal averaging is required in this case, no phase cycling was performed, and given a high enough signal to noise, this measurement can be made in a single scan. Using a higher field strength, this can be achieved.

\subsection{High Field Measurements Using the Modified Difftrain Pulse Sequence}

\subsubsection{Introduction}

In this section the modified Difftrain pulse sequence as described in subsection 5.3.5 is implemented on the $1.5 \mathrm{~T}$ superconducting geometry NMR system to make a true single scan, single-shot surface to volume ratio measurement for a well defined porous medium. 
This result provides a proof of concept for real-time surface to volume ratio measurements, as well as multiple molecular mobility measurements in a single scan which can all be correlated back to a single event in time.

\subsubsection{Sample Characterisation}

The sample used for restricted diffusion measurements and a true single-shot surface to volume ratio measurement in the $1.5 \mathrm{~T}$ superconducting magnet was a $24 \mathrm{~mm}$ long, 18 mm wide cylinder filled with 215-250 $\mu$ m polyethylene beads (Cospheric), with the pore space saturated with methanol. The sample was oriented such that the axis of the sample cylinder was centred and aligned along the $z$ axis. This sample geometry and alignment was chosen such that a uniform spin density exists in the $z$ direction, and when applying a $z$-read gradient, a Gaussian can again be fit directly to the images to perform diffusion measurements. The same process used to measure the porosity and predict the surface to volume ratio of the pore space was used as described for the low field samples. The porosity was measured again as $\phi=0.36 \pm 0.01$. This yields a predicted surface to volume ratio of $4.1 \times 10^{4} \mathrm{~m}^{-1} \leq S / V \leq 5.2 \times 10^{4} \mathrm{~m}^{-1}$.

\subsubsection{Short Time Limit Restricted Diffusion Measurements}

The 13-Interval modified Difftrain pulse sequence as previously described in subsection 5.3.5 and illustrated in Fig. 5.5, is again shown in Fig. 7.12. This pulse sequence was run for the bead pack described in subsection 7.4.2 using experimental parameters of $C=9 \mathrm{Tm}^{-2}, \delta=4 \mathrm{~ms}$, observation times at multiples of $30 \mathrm{~ms}$, a $z$-read gradient of $11.7 \mathrm{mT} / \mathrm{m}, 50 \mu \mathrm{s}$ dwell time, and 20 points taken across the sample. These parameters give a maximum diffusion length of $d_{l}=26 \mu \mathrm{m}$ and a characteristic length scale of $l_{c}=456 \mu \mathrm{m}$, making $l_{c} \gg d_{l}$, and the condition in Eq. 5.22 holds with $z$ replacing $y$ due to the use of superconducting geometry instead of Halbach geometry. This results in the relationship from Eq. 7.1 between the normalised read image normalised and the diffusion coefficient represented in terms of $z$ such that

$$
E_{N}(z)=\exp \left\{-(\gamma \delta C z)^{2} D(\Delta-\delta / 3)\right\}
$$


For this experiment, the observation times were increased from those used for the low field experiments due to the complexity of the modified pulse sequence, eddy currents, and strong coupling between the RF coil and the second order field coil. When the RF

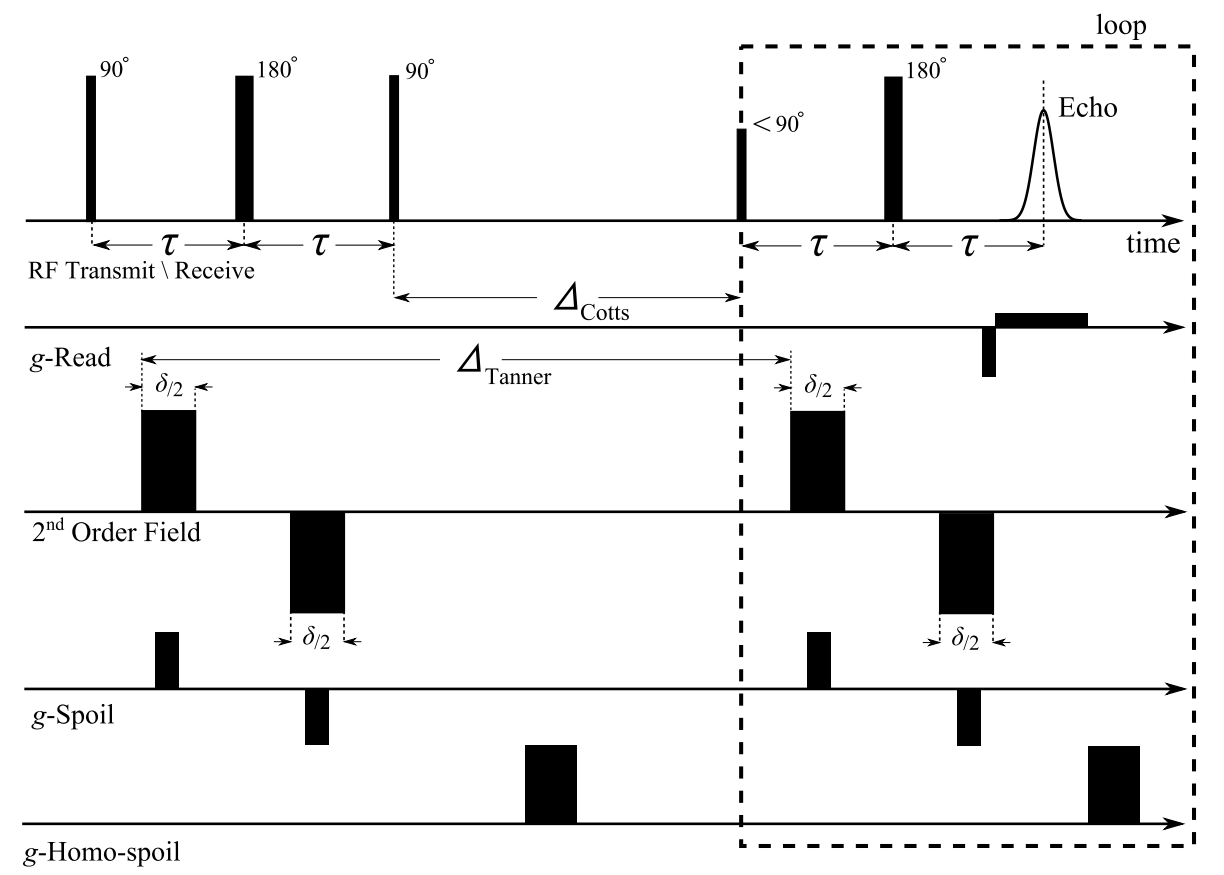

Figure 7.12: Modified Difftrain pulse sequence for a single-shot measurement of $D_{a p p}(\Delta)$ at multiple $\Delta$ with incorporation of a 13-interval pulse sequence.

coil was pulsed, the close proximity between the RF and second order field coil resulted in a coupling which induced a DC response from the amplifier driving the second order coil. A thin sheet of copper was placed on the inner surface of the second order coil former, grounded, and insulated from the RF coil to form an RF shield and minimise this effect. Long $(1 \mathrm{~ms})$, weak RF pulses were also used to reduce this response by over an order of magnitude. The remaining coupling between the second order and $\mathrm{RF}$ coil, and eddy currents from the multiple spoiler, RF, and second order field pulses resulted in necessary gradient pulse ramping times and delays between gradient and $\mathrm{RF}$ pulses which prevented shorter observation times for reliable performance of the pulse sequence. For these reasons, the observation times were set at multiples of $30 \mathrm{~ms}$, a safe and conservative value for operation of the pulse sequence, and still places the last of 5 
diffusion measurements just at the limit of the short time approximation for a surface to volume ratio measurement.

The bead pack was loaded into the second order and RF coil insert (Fig. 6.14), placed in the magnet, and the experiment performed with 1 scan. In the left panel of Fig. 7.13, the reference and diffusion encoded images are shown. The pulse sequence was run first without the application of the second order field pulses, and the resulting reference images shown as dashed curves. The pulse sequence was then run with the second order field pulses to obtain the diffusion encoded images shown as solid curves. By fitting a Gaussian curve to these images, the apparent diffusion coefficients are obtained for the corresponding observation times. These diffusion measurements were plotted and a linear

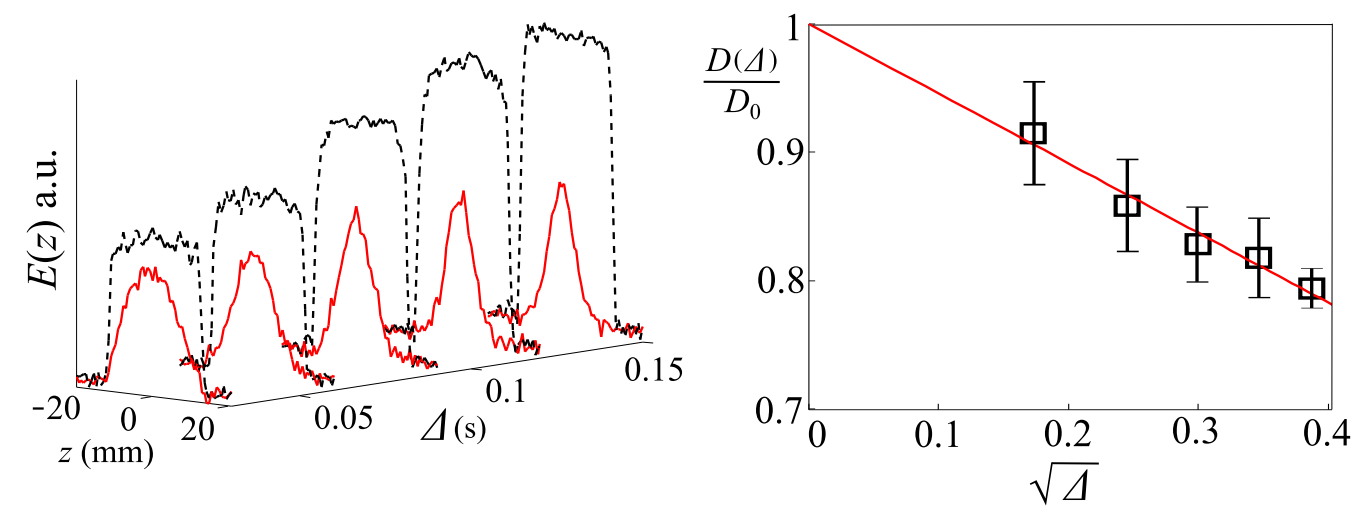

Figure 7.13: Measurement of the apparent diffusion coefficient in the short limit for a 215-250 $\mu \mathrm{m}$ bead pack. 5 echoes are acquired to provide 5 measurements of $D_{\text {app }}(\Delta)$. Left: Images acquired from the 5 stimulated echoes. The dashed lines show the reference images obtained in the absence of the second order field pulses. The solid lines show the diffusion encoded images which experience attenuation due to the second order field pulses. Right: The diffusion measurements are plotted against $\sqrt{\Delta}$, and a linear fit made to approximate the surface to volume ratio of the bead pack.

fit made to the data to estimate a surface to volume ratio of $4.3 \times 10^{4} \mathrm{~m}^{-1}$ with an error of $\pm 0.4 \times 10^{4} \mathrm{~m}^{-1}$ fitting strictly to the data points, and error $\pm 2.5 \times 10^{4} \mathrm{~m}^{-1}$ calculated using the error bars for each diffusion measurement (right Fig. 7.13). The measured value is in good agreement with the predicted value for both cases. This measurement was performed in a single scan, single-shot experiment, remarkably, with a total experimental 
time and temporal resolution of only $150 \mathrm{~ms}$.

\subsection{High Field Single-Shot Propagator Measurements}

\subsubsection{Introduction}

In this section the single-shot propagator measurement as described in section 5.4 is implemented on the $1.5 \mathrm{~T}$ superconducting geometry NMR system. Average propagator measurements are performed in real-time with a single scan for each average propagator measurement for various flow rates through a cylindrical tube. These results present a proof of concept for real-time characterisation of flow with temporal resolution of tens of milliseconds, and verify the phase evolution, complex mapping, and echo condition theories derived in section 5.4.

\subsubsection{Hardware and Experimental Set-Up}

A $1 \mathrm{~m}$ long glass pipe of inner diameter $18 \mathrm{~mm}$ was run through the second order and RF coil insert, and the entire apparatus loaded into the $1.5 \mathrm{~T}$ superconducting magnet (Fig. 7.14). The length of pipe was centred in the system such that the middle of the pipe was

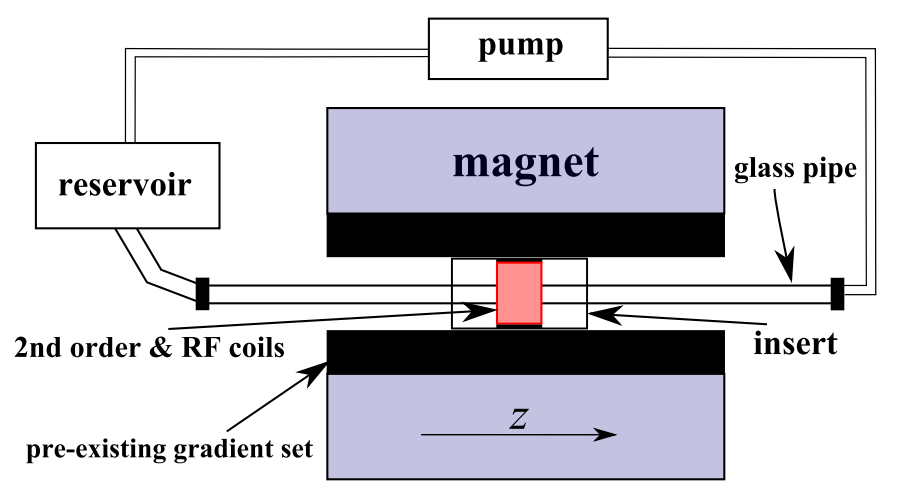

Figure 7.14: Experimental set-up for flow through experiments, and cross section of the magnet. The pump circulates the water through the glass pipe. The glass pipe runs through the magnet, existing gradient stack, and the second order field and RF coil insert. 
located in the RF coil. The entrance length $L_{e}$ defines the distance at which laminar flow is developed from the entrance of the pipe. This length can be approximated by $L_{e} \approx a R e$, where $a$ is the radius of the pipe, and $R e$ is the Reynold's number. Using a maximum flow rate of $50 \mathrm{~mL} / \mathrm{min}$, and a pipe radius of $9 \mathrm{~mm}$, it is calculated that $L_{e} \approx 0.53 \mathrm{~m}$, and the assumptions used for the theoretical propagator calculation from Eq. 2.21 will hold. This maximum flow rate corresponds to an average velocity of $3.3 \mathrm{~mm} / \mathrm{s}$, and a peak velocity at the centre of the pipe of $6.5 \mathrm{~mm} / \mathrm{s}$. A pump was connected to the outflow of the pipe, and a reservoir attached to the inflow of the pipe. The outflow of the pump was fed back into the reservoir. The pre-existing gradient stack used for slice selection and the read gradient is also shown in the cross sectional diagram of the magnet in Fig. 7.14.

\subsubsection{Verifying Experimental Requirements for the Echo as the Propagator}

In order for the echo to be correctly interpreted as the propagator with a mapping from time to displacement space, the afore mentioned conditions in subsection 5.4.8 must be fulfilled. Firstly, full attenuation of the encoded read image be must be attained within the homogeneous regions of the magnetic fields. To verify this, a cylindrical distilled water sample of inner diameter $18 \mathrm{~mm}$ and length $22 \mathrm{~mm}$ was placed in the coil insert with its axis centred and aligned with the $z$ axis, and imaged with the pulse sequence previously illustrated in Fig. 5.6, shown again in Fig. 7.15, with experimental parameters set to $C=16 \mathrm{Tm}^{-2}, \delta=5 \mathrm{~ms}, \Delta=35 \mathrm{~ms}$, and an $x$-slice thickness of $F W H M=2.67 \mathrm{~mm}$. In Fig. 7.16, a 1D reference read image of the slice without the second order field pulses is shown as a dashed line. The image provides an accurate profile of the sample, showing the sample resides in a homogeneous region of the magnetic fields. The encoded read image resulting from the pulse sequence when run with the second order field pulses is shown as a solid line. Full diffusive attenuation of the encoded read image is achieved at the edge of the sample, and within the homogeneous regions of the fields.

The amplitude of the encoded read image at $z=0$ shows no apparent attenuation with respect to the reference read image. Since the attenuation experienced by the encoded 


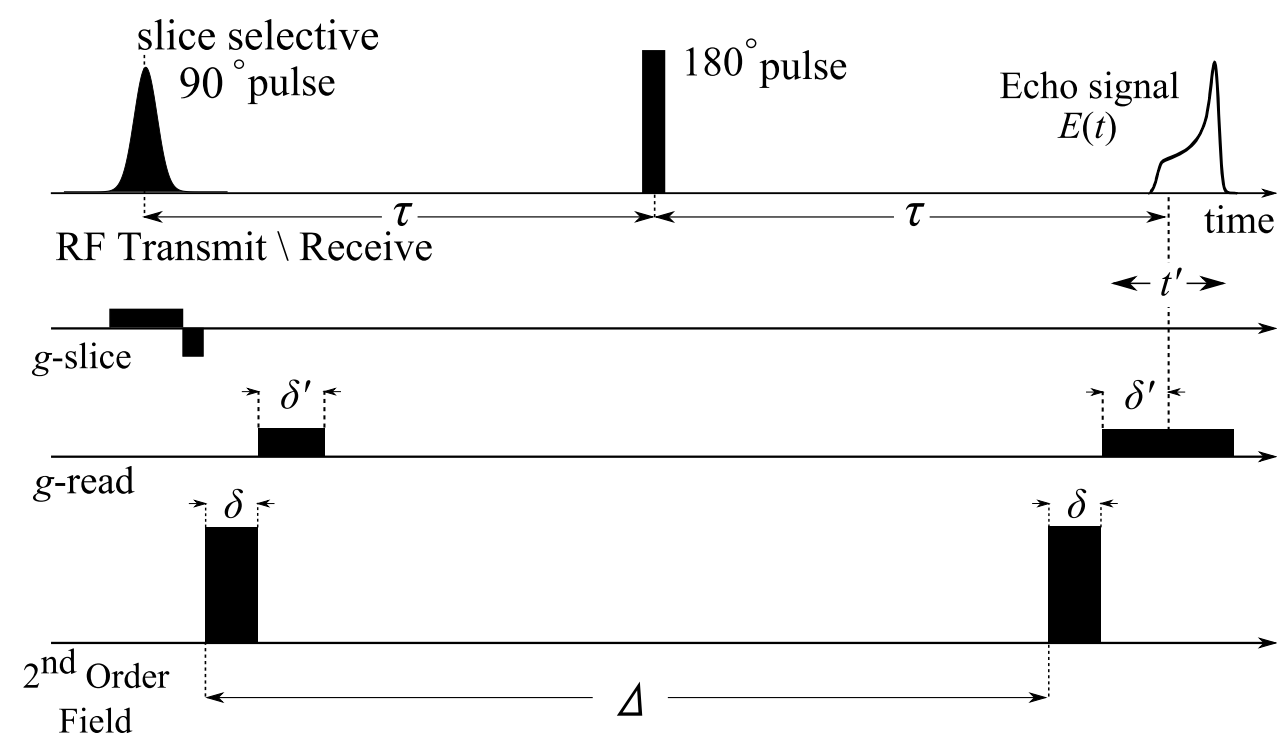

Figure 7.15: Single-shot PGSE-imaging sequence for propagator measurement.

read image is due to diffusion, it will obey the relationship

$$
E(z) \propto \exp \left\{-(\gamma \delta g)^{2} D(\Delta-\delta / 3)\right\}
$$

The gradient strength due to the pulsed second order field can be expressed as

$$
g=C \sqrt{x^{2}+z^{2}} .
$$

The lack of attenuation at $z=0$ in Fig. 7.16 shows that no significant gradient strengths have been sampled across the slice thickness in the $x$ direction, and the gradient strength $g$ should be a function of $z$ alone such that

$$
g \approx C z
$$

This is an indicator that the mapping between real space and $q$-space is well defined, and therefore the mapping between time and displacement space is also well defined. With all conditions met, the echo will represent the average propagator for these experimental parameters. 


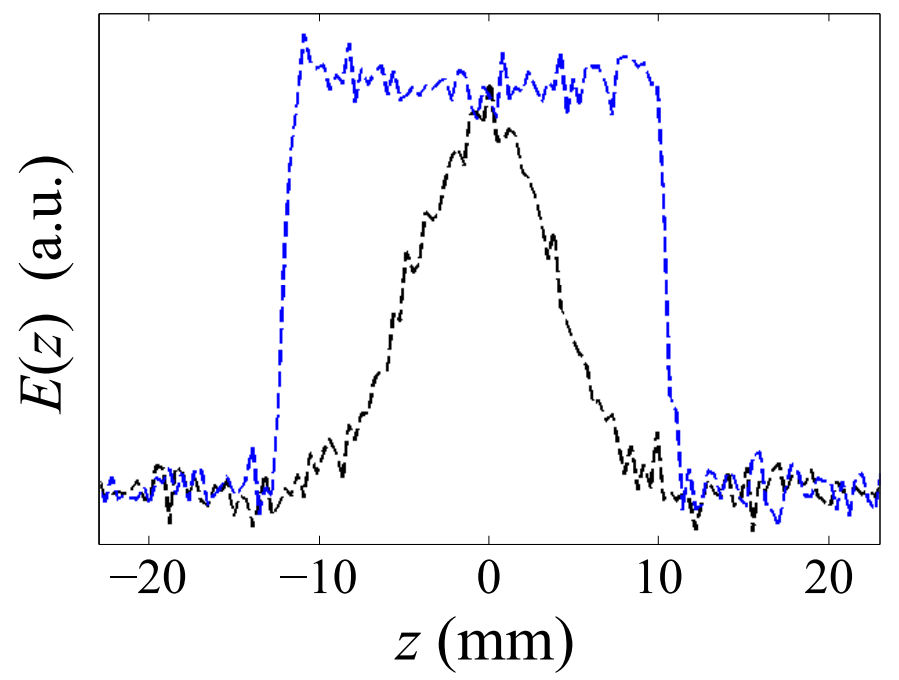

Figure 7.16: Reference read image without second order field pulses (dashed blue), and encoded read image with second order field pulses (dashed black), of an $18 \mathrm{~mm}$ diameter, $22 \mathrm{~mm}$ long, cylindrical distilled water sample. Full attenuation of the image is achieved at the edge of the sample, and no diffusive attenuation is apparent at the centre of the sample.

This process is crucial to performing the flow experiments. Once a long pipe filled with water is introduced, the sample will extend far into the inhomogeneous regions of the magnetic fields, and the resulting image distortions will make it difficult to determine whether or not full attenuation of the image occurs within the homogeneous regions of the coils.

In Fig. 7.17, the encoded read image from the water filled pipe (solid red curve) and the cylindrical water sample (dashed black curve) are plotted on top of one another. As expected, because the same experimental parameters have been used, they appear identical. Since the encoded read image from the cylindrical sample did not experience any diffusive attenuation at $z=0$ with respect to its reference image, the second order field experienced by the slice for the water filled pipe can be well approximated by a parabolic field, and the echo will represent the average propagator. 


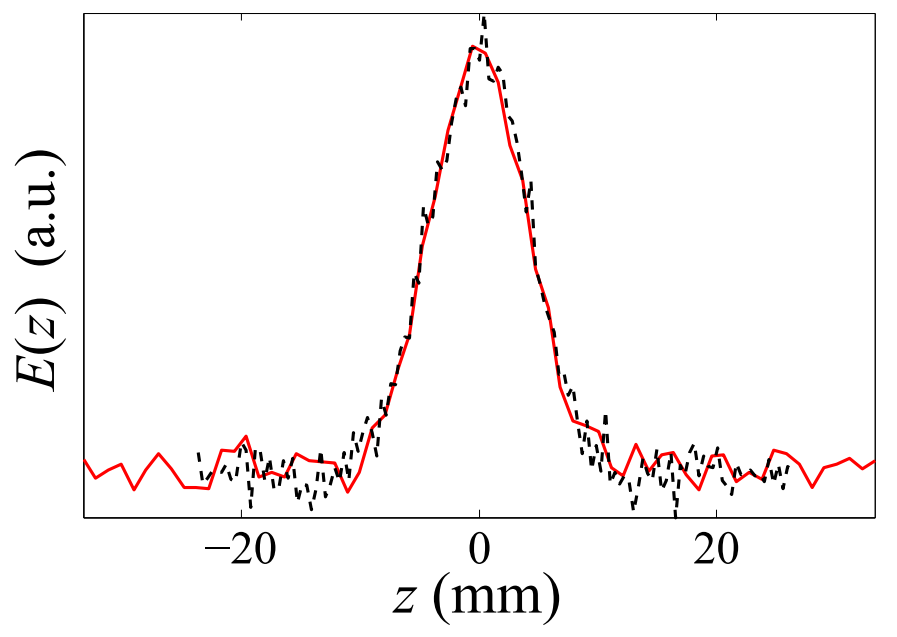

Figure 7.17: The encoded read images for the cylindrical water sample (dashed black) and water filled pipe (solid red) are plotted together. As expected, the images are identical, showing attenuation of the encoded image is achieved within the homogeneous regions of the fields.

\subsubsection{Propagator Measurements for Ramped Flow}

This technique was verified using pipe flow of water through the NMR system. The experiment was performed by acquiring a single echo for each flow rate, with the flow rate ramped from 0 to $50 \mathrm{~mL} / \mathrm{min}$. The echoes were phased with the time dependent third order phase correction from Eq. 5.58, and the real component of the echo plotted as a series in the left of Fig. 7.18. The theoretical propagators for the corresponding flow rates are plotted along side this in the right of Fig. 7.18, and agree well with the experimental results. To better illustrate the validity of this technique, individual echoes for various flow rates from the series in Fig. 7.18 are plotted as solid lines, with the theoretical propagators as dashed lines in Fig. 7.19. Here, both the time and displacement space axes are plotted in the horizontal direction using the relationship between time and displacement from Eq. 5.39, and the probability for displacement in the vertical direction in arbitrary units.

The echo shown in Fig. 7.19 for a flow rate of $0 \mathrm{~mL} / \mathrm{min}$ had no phase correction applied, but still agrees with the theoretically predicted average propagator. This is due to only small displacements $Z$ occurring where $l_{c} \gg|Z|^{\text {max }}$, and proper experimental set-up 

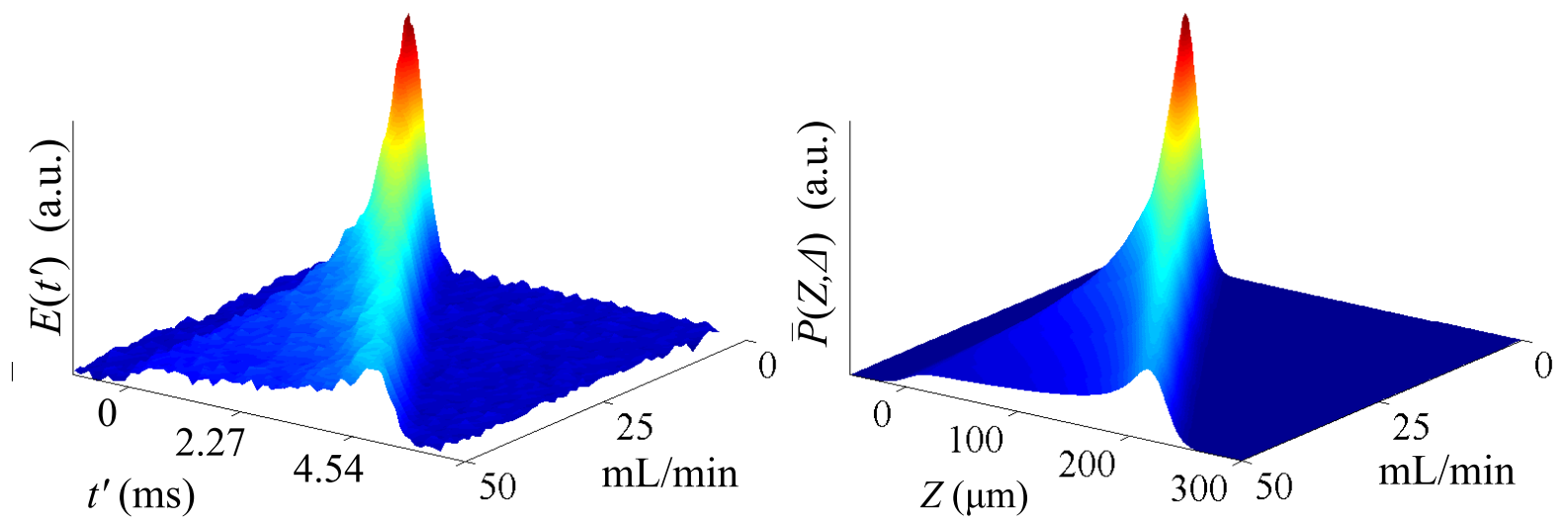

Figure 7.18: Left: A series of echo signals $E\left(t^{\prime}\right)$ phased with the phase correction from Eq. 5.58, and plotted against time $t^{\prime}$ for flow rates ranging from 0 to $50 \mathrm{~mL} / \mathrm{min}$. Each echo was acquired in a single scan. Right: Theoretical average propagators are plotted against displacement $Z$ for flow rates ranging from 0 to $50 \mathrm{~mL} / \mathrm{min}$.

which ensures the receiver is set on resonance and in phase with the echo signal. As the flow rate is increased, a time dependent phase shift will occur across the echo signal, and as shown in Fig. 7.19, the phase mapping from Eq. 5.58 appears to provide the appropriate correction. To further investigate the validity of the time dependent phase correction from Eq. 5.58, signal averaging of the echo signal for the largest flow rate is performed in the next subsection. 


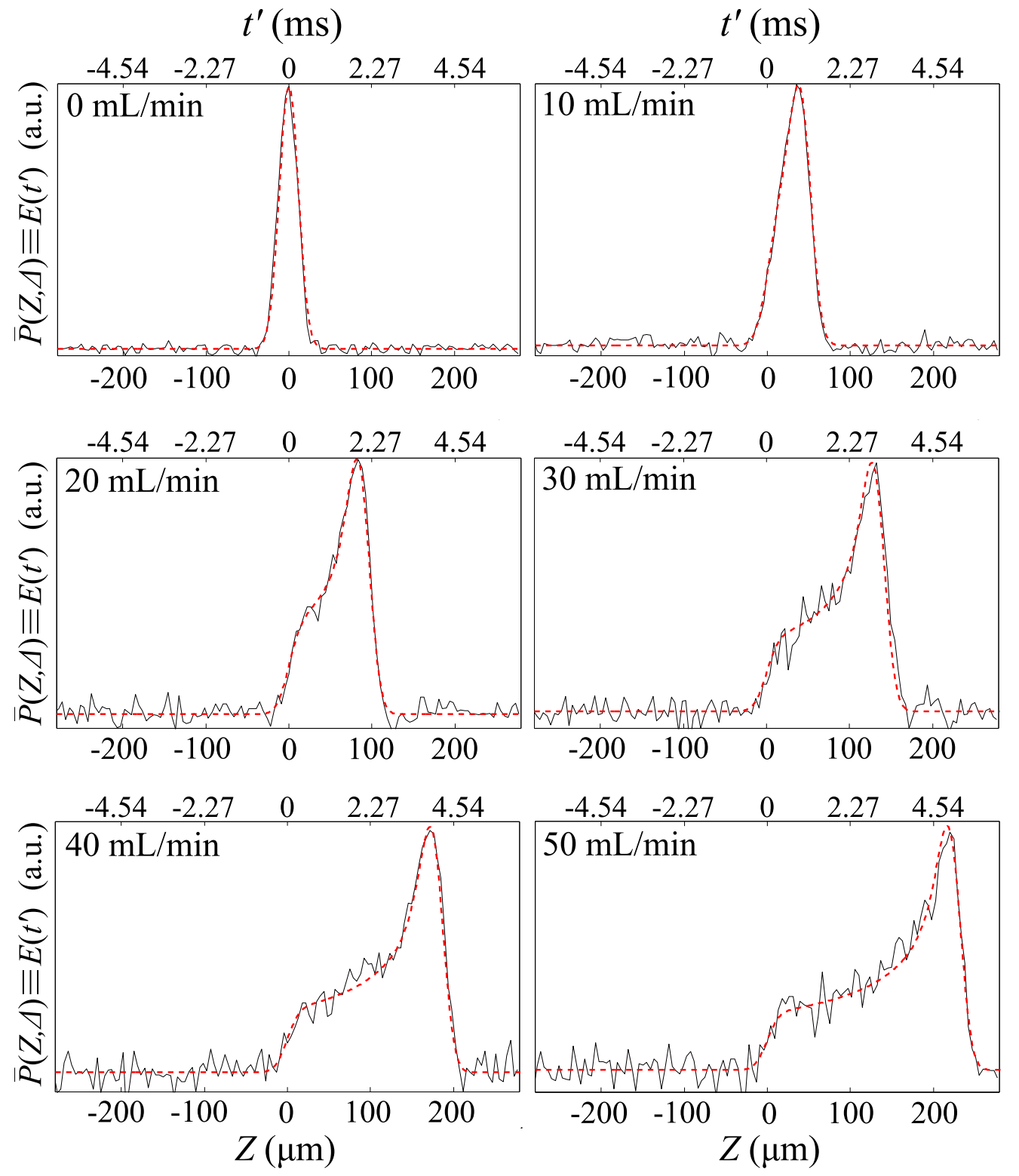

Figure 7.19: Echoes resulting from the single-shot propagator measurement illustrated in Fig. 7.15 for selected flow rates from Fig. 7.18. The solid lines represents the measurements, and the dashed lines the theoretical propagators for the given flow rates. The real part of the echo data $E\left(t^{\prime}\right)$ is plotted against time $t^{\prime}$ at the top of each plot after applying the phase correction from Eq. 5.58, and also against displacement $Z$ using the mapping from Eq. 5.39 at the bottom of each plot. Under the time to displacement mapping, the echo signal $E\left(t^{\prime}\right)$ becomes the average propagator $\bar{P}(Z, \Delta)$ shown on the vertical axis. 


\subsubsection{Complex Mapping for Large Displacements}

If $l_{c} \gg|Z|^{\text {max }}$, the mapping between time and displacement space will be real, but if not, the full complex mapping can be used to obtain a purely real valued propagator. The value of $|Z|^{\max }$ for a given flow rate is obtained by considering a particle which is moving at the maximum velocity at the centre of the pipe, and has also undergone a displacement due to diffusion in the same direction as the flow. For laminar flow through a pipe and a parabolic flow velocity profile, the maximum velocity $v_{0}$ at the centre of the pipe is given by

$$
v_{0}=2 \bar{v}
$$

where $\bar{v}$ is the mean velocity, which is equal to the volume flow rate divided by the cross sectional area of the pipe. Using a maximum flow rate of $50 \mathrm{~mL} / \mathrm{min}$, the maximum velocity is multiplied by the observation time $\Delta$, and adding displacement due to diffusion of the diffusion length $d_{l}=\sqrt{2 D \Delta}$, the maximum displacement without dispersion is found to be

$$
|Z|^{\max }=2 \bar{v}+\sqrt{2 D \Delta} \approx 241 \mu \mathrm{m}
$$

The experimental parameters yield a value of $l_{c}=306 \mu \mathrm{m}$, and $l_{c} \ngtr|Z|^{\text {max }}$. Therefore, it is expected that the full complex mapping between time and displacement space will be necessary for such a flow rate.

To test the validity of the phase mapping approximation from Eq. 5.58, 128 scans were taken without phase cycling to signal average the echo for a flow rate of $50 \mathrm{~mL} / \mathrm{min}$. The unphased complex echo signal from this experiment is shown in Fig. 7.20a. The solid curve represents the real part, and the dashed curve the imaginary part. In Fig. 7.20b, the phase of the echo is shown as a solid curve, and the theoretical phase approximation from the third order polynomial in Eq. 5.58 shown as a dashed curve.

The approximated phase mapping (Eq. 5.58) and time to displacement space mapping (Eq. 5.39) were applied to Fig. 7.20a to obtain the propagator shown in Fig. 7.20c. The solid curve represents the real part after the phase mapping, and the dashed curve represents the imaginary part. The data points in Fig. 7.20c represent the theoretical propagator. The phase of Fig. 7.20c is shown in Fig. 7.20d. As illustrated, the phase 

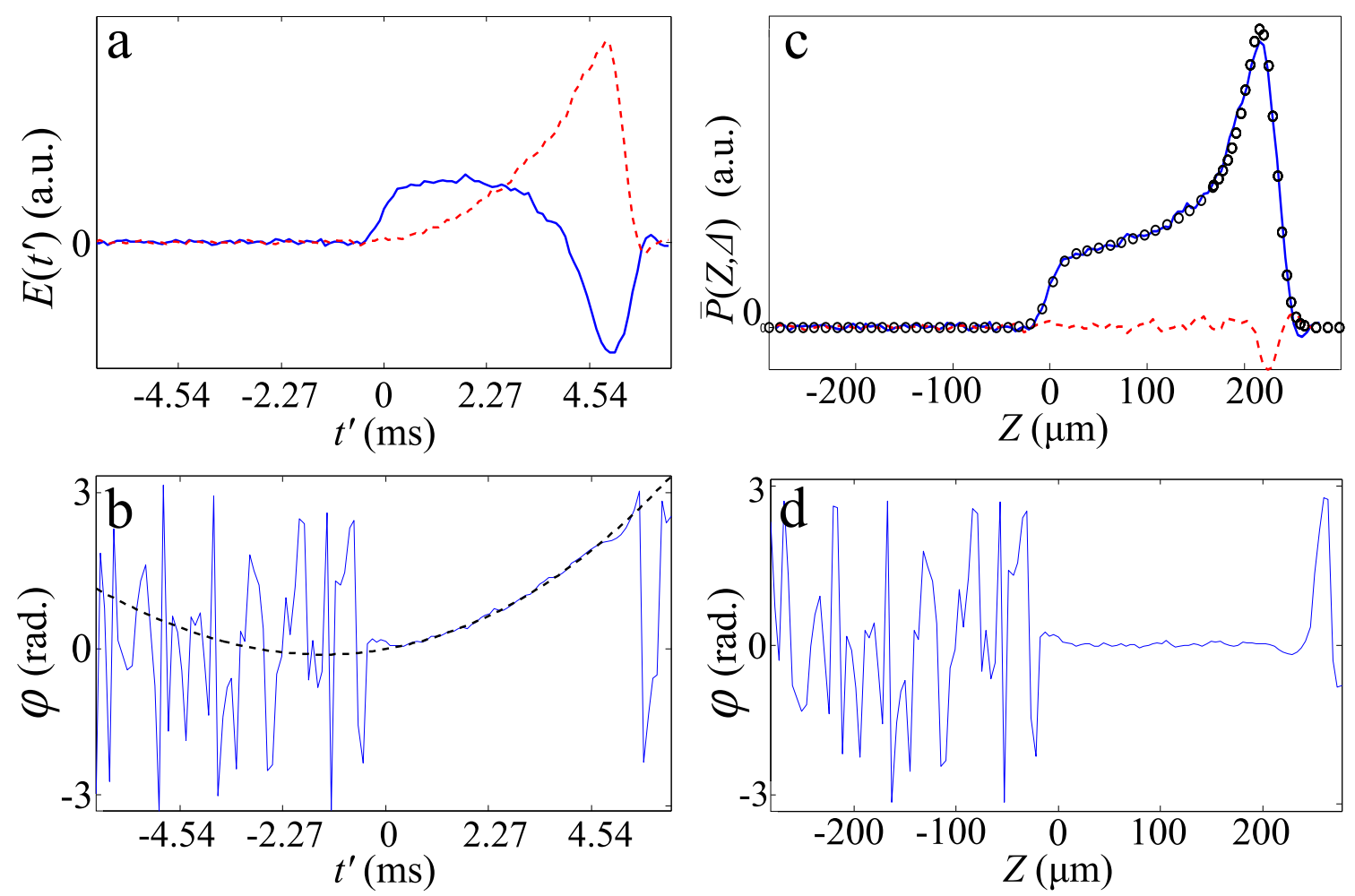

Figure 7.20: a: The unphased echo signal for a flow rate of $50 \mathrm{~mL} / \mathrm{min}$. showing a time dependent phase shift. The dashed curve is the imaginary part of the echo signal, and the solid curve is the real part. b: The phase of the echo from panel-a is shown as a solid curve, and the approximated time dependent phase shift from Eq. 5.58 is shown as a dashed curve. c: The phased echo after applying the phase correction from Eq. 5.58. The real part of the phased echo is shown as a solid curve, the imaginary part as a dashed curve, and the theoretically calculated propagator from Eq. 2.21 as circles. The approximation accurately phases the echo for displacements within the flow regime where $0 \leq Z \leq v_{0} \Delta$. d: The phase of the corrected echo. As shown in panel-c, the phase is now equal to zero, and the signal entirely real for displacements within the flow regime. 
correction under the assumption of constant velocity works well within the flow regime $0 \leq Z \leq v_{0} \Delta$, where $v_{0} \Delta$ is calculated to be $215 \mu \mathrm{m}$ for the measured flow rate.

The ability of this method to present the average propagator directly in the time domain without the use of any Fourier transforms in remarkable. It also allows an increase in displacement resolution without affecting the temporal or spatial resolution of the measurement by simply decreasing the dwell time per data point in the time domain and increasing the number of data points acquired. Of course there must be some drawback from this result. The drawback from this increased resolution is an increase in the bandwidth of noise allowed into the receiver coil, which roughly scales as the square root of the bandwidth, which is still preferable to imaging techniques which result in a linear decrease in signal to noise for increased spatial resolution. When compared to conventional PGSE, this method is very advantageous as experimental time for conventional PGSE typically scales linearly with the number of data points desired for displacement resolution, with the repetition time for each consecutive data point limited by $T_{1}$ relaxation. 



\section{Chapter 8}

\section{Conclusion}

\subsection{Results Presented in this Thesis}

\subsubsection{Parallel Acquisition of $q$-Space}

A proof of concept for the parallel acquisition of $q$-space data has been presented for both flow and diffusion. Although simple systems were analysed, this was of great importance in testing this technique as accurate theoretical predictions provided a comparison for the experimental results, and a method for validating the accuracy of the proposed techniques. The second order field coil fabrication and characterisation was vital in applying a field whose gradient strength varied linearly with respect to position. Care was taken not only in designing the coil to well defined specifications, but also in characterisation of its field to ensure experiments were performed and interpretation of results were made in a justifiable and correct fashion.

The mapping between real space and $q$-space also relied on precise spatial encoding through pulsing first order magnetic field gradient coils. These coils were also designed, fabricated, and characterised such that data analysis and interpretation of results held under the theory derived in this thesis. 


\subsubsection{Diffusion Measurements}

The diffusion measurements presented in this thesis were developed in a sequential process where complexity of the experiment and sample were increased in an iterative manner to ensure all methods performed well under scrutiny of their building blocks.

Free diffusion for a single-shot experiment was first performed for a mapping between real space and $q$-space. With precise measurements made for signal averaged experiments, relaxation of the slice selection requirement revealed the diffusion measurement could still be made without a slice selection. This agreed with the theory derived for the diffusion encoded image in the absence of slice selection.

Implementation of these methods into Difftrain without slice selection was performed after the basic case of single-shot diffusion had been rigorously tested. The modified Difftrain technique was then tested using ten echoes for free diffusion of water, giving the expected result of an apparent diffusion coefficient which is independent of observation time. With this proof of concept, restricted diffusion measurements were made on well characterised samples, with all experimental results shown to agree well with theoretically predicted values of tortuosity and the surface to volume ratio. This section of the research was concluded with a true single-shot surface to volume ratio measurement performed in real-time with one single scan.

The measurement limitations of the hardware used for single-shot diffusion measurements in this thesis will, in part, depend on the curvature $C$ of the second order field, a property determined by the second order field coil and the amplifier driving it. Since the smallest mean squared displacements, and hence smallest diffusion coefficients, are measured using the largest gradients, and the curvature of the second order field limits the maximum gradient applied, increasing the curvature will result in the ability to measure smaller diffusion coefficients. To achieve a higher second order field curvature, a stronger current amplifier could be used, the practical limit being on the order of $100 \mathrm{~A}$, as well as building a second order coil of a much higher curvature per amp. Such a system would most likely be smaller in size than that which is presented in this thesis, and would benefit from a stronger $\mathbf{B}_{0}$ field to increase the available signal to noise. 


\subsubsection{Propagator Measurements}

The average propagator was first measured for free diffusion using a thin slice selection for water by acquiring the spin echo signal and mapping from time to displacement space. The echo yielded an average propagator which was in excellent agreement with the theoretical propagator calculation. With the most basic proof of concept performed, flow propagators were then measured.

The flow propagator measurements were made in a long cylindrical pipe, with flow ramped from 0 to $50 \mathrm{~mL} / \mathrm{min}$. Before making the average propagator measurements, using a finite sample contained well within the homogeneous regions of all magnetic fields, care was taken to define experimental parameters which ensured the echo would accurately represent the average propagator. With the first average propagator measurement made for no flow, the echo which yielded the average propagator was analysed to again give the known diffusion coefficient for water. The flow rate was then increased, with each echo yielding the average propagator for corresponding experimental conditions. Theory was developed for a calculation of the expected average propagator for the selected slice volume of flow. When the theoretical propagators were compared with the experimental measurements, they were found to be in excellent agreement.

\subsection{Future Work}

\subsubsection{Different Samples and Systems}

This method can be applied to any system or sample which is homogeneous on the length scale of the encoded image pixel width. Some steady-state applications would include diffusive and flow diffraction $[9,55,117]$ and magnetic resonance pore imaging (MRPI) $[11,33]$ for determination of the structure factor of the porous medium, dispersion measurements $[5,6,8,9,15,18,65]$, as well as measurement of the non-local dispersion tensor $[26,118,119]$.

To enable single-shot surface to volume ratio or tortuosity measurements in low field systems, hyperpolarised fluids could be used to increase the signal to noise available, potentially alleviating the need for signal averaging. Hyperpolarised fluids can also experience 
polarisation fluctuations in time, making reference measurements necessary such that diffusive attenuation can be measured against the current level of polarisation throughout a series of experiments. With all diffusion data acquired in a single scan for the methods presented in this research, the polarisation level is constant across the diffusion data, and no reference measurements would be necessary.

The high temporal resolution available with all techniques presented in this thesis could allow characterisation of systems which are changing rapidly in time such as average propagators for pulsed flow, unsteady flow fields, start-up flow, and transient behaviours in non-Newtonian fluids. The rapid measurement of the surface to volume ratio and tortuosity provides a method for characterising porous media where the pore boundaries are evolving in time. Such media would include emulsions, systems where pulsed flow and high pressure may affect the geometry of the pore space, and biological systems where cells are growing, dying, or are affected by the introduction of some external variable such as a chemical, light, or protein which induces a time dependent behaviour.

\subsubsection{Different Methods and Techniques}

This method of mapping real space to $q$-space can be applied to any NMR measurement technique in which $q$-space data is required, as long as the sample is homogeneous on the length scale of the pixel width. Since all displacement information is encoded into a single spin echo, echo trains could be utilised both for enhancement in signal to noise of the measurement and also for multidimensional encoding. Using multiple $180^{\circ} \mathrm{RF}$ pulses to form an echo train, and the echo acquired during a read gradient, echoes could be summed to enhance the signal to noise for each measurement. An echo train after the formation of a diffusion encoded image could also be used to make a two dimensional $D-T_{2}$ map using an inverse Laplace transformation.

Another possibility is measuring distributions of diffusion coefficients and the use of higher order pulsed fields. If a fourth order field coil was built and pulsed, a larger range of gradient strengths could be applied in a single pulse compared to a pulsed second order field. This could allow a large range of diffusion coefficients to be measured in a single-shot experiment. 


\subsection{Final Thoughts}

The ability of second order magnetic fields to transform the acquisition of $q$-space data from a serial process to a parallel process results in an extremely fast measurement technique allowing real-time fluid transport measurements. One of the best results of pulsing second order fields is that a single, well defined observation time is used, and gradient strengths ranging from zero to a value inducing complete diffusive signal attenuation are available in one single pulse. In turn, this has enabled the first single-shot surface to volume ratio measurement and first single-shot average propagator measurement. For the single-shot surface to volume and average propagator measurements, and the parallel acquisition of $q$-space data in general, a decrease in experimental time of orders of magnitude has been achieved while increasing the displacement resolution. This should enable the characterisation and real-time monitoring of processes which could not previously be resolved. 



\section{Bibliography}

[1] J. Mitchell and M. L Johns. Rapid measurements of diffusion using PFG: Developments and applications of the Difftrain pulse sequence. Concepts in Magnetic Resonance Part A, 34A(1):1-15, January 2009.

[2] R. W. Mair, P. N. Sen, M. D. Hürlimann, S. Patz, D. G. Cory, and R. L. Walsworth. The Narrow Pulse Approximation and Long Length Scale Determination in Xenon Gas Diffusion NMR Studies of Model Porous Media. Journal of Magnetic Resonance, 156(2):202-212, June 2002.

[3] R. W. Mair, G. P. Wong, D. Hoffmann, M. D. Hurlimann, S. Patz, L. M. Schwartz, and R. L. Walsworth. Probing porous media with gas diffusion NMR. Physical Review Letters, 83(16):3324-7, October 1999.

[4] S. Capuani, M. Alesiani, F. M. Alessandri, and B. Maraviglia. Characterization of porous media structure by non linear NMR methods. Magnetic resonance imaging, 19(3-4):319-23, 2001.

[5] B. Manz, P. Alexander, and L. F. Gladden. Correlations between dispersion and structure in porous media probed by nuclear magnetic resonance. Physics of Fluids, 11(2):259, 1999.

[6] B. Manz, L. F. Gladden, and P. B. Warren. Flow and dispersion in porous media: Lattice-Boltzmann and NMR studies. AIChE Journal, 45(9):1845-1854, September 1999.

[7] L. F. Gladden and J. Mitchell. Measuring adsorption, diffusion and flow in chemical engineering: applications of magnetic resonance to porous media. New Journal of Physics, 13(3):035001, March 2011.

[8] J. D. Seymour and P. T. Callaghan. Generalized approach to NMR analysis of flow and dispersion in porous media. AIChE Journal, 43(8):2096-2111, August 1997. 
[9] J. D. Seymour and P. T. Callaghan. "Flow-Diffraction" Structural Characterization and Measurement of Hydrodynamic Dispersion in Porous Media by PGSE NMR. Journal of Magnetic Resonance, Series A(122):90-93, 1996.

[10] N. Spindler, P. Galvosas, A. Pohlmeier, and H. Vereecken. NMR velocimetry with 13-interval stimulated echo multi-slice imaging in natural porous media under low flow rates. Journal of Magnetic Resonance, 212(1):216-23, September 2011.

[11] S. Hertel, M. Hunter, and P. Galvosas. Magnetic resonance pore imaging, a tool for porous media research. Physical Review E, 87(3):030802, March 2013.

[12] P. P. Mitra, P. N. Sen, and L. M. Schwartz. Short-time behavior of the diffusion coefficient as a geometrical probe of porous media. Physical Review B, 47(14):85658574, 1993.

[13] P. P. Mitra, P. N. Sen, L. M. Schwartz, and P. Le Doussal. Diffusion propagator as a probe of the structure of porous media. Physical Review Letters, 68(24):3555-3558, 1992.

[14] L. L. Latour, P. P. Mitra, R. L. Kleinberg, and C. H. Sotak. Time-Dependent Diffusion Coefficient of Fluids in Porous Media as a Probe of Surface-to-Volume Ratio. Journal of Magnetic Resonance, Series A, 101(3):342-346, February 1993.

[15] D. L. Koch and J. F. Brady. A non-local description of advection-diffusion with application to dispersion in porous media. Journal of Fluid Mechanics, 180:387-403, April 1987.

[16] P. J. Barrie. Characterization of Porous Media With NMR Methods. Annual Reports on NMR Spectroscopy, 41:265-316, 2000.

[17] L. L. Latour, R. L. Kleinberg, P. P. Mitra, and C. H. Sotak. Pore-Size Distributions and Tortuosity in Heterogeneous Porous Media. Journal of Magnetic Resonance, Series A, 112(1):83-91, January 1995.

[18] A. A. Khrapitchev and P. T. Callaghan. Reversible and irreversible dispersion in a porous medium. Physics of Fluids, 15(9):2649, 2003.

[19] D. Bergman and K. Dunn. NMR of diffusing atoms in a periodic porous medium in the presence of a nonuniform magnetic field. Physical Review E, 52(6):6516-6535, December 1995. 
[20] C. Vogt, P. Galvosas, N. Klitzsch, and F. Stallmach. Self-diffusion studies of pore fluids in unconsolidated sediments by PFG NMR. Journal of Applied Geophysics, 50(4):455-467, July 2002.

[21] R. W. Mair, M. S. Rosen, R. Wang, D. G. Cory, and R. L. Walsworth. Diffusion NMR methods applied to xenon gas for materials study. Magnetic Resonance in Chemistry, 40(13):S29-39, December 2002.

[22] C. J. Davies, J. D Griffith, A. J. Sederman, L. F. Gladden, and M. L Johns. Rapid surface-to-volume ratio and tortuosity measurement using Difftrain. Journal of Magnetic Resonance, 187(1):170-5, July 2007.

[23] P. N. Sen. Time-dependent diffusion coefficient as a probe of geometry. Concepts in Magnetic Resonance, 23A(1):1-21, September 2004.

[24] B. M. Goodson. Nuclear magnetic resonance of laser-polarized noble gases in molecules, materials, and organisms. Journal of Magnetic Resonance, 155(2):157216, April 2002.

[25] L. F. Gladden. Magnetic Resonance : Ongoing and Future Role in Chemical Engineering Research. AIChE Journal, 49(1):2-9, 2000.

[26] M. Hunter and P. Callaghan. NMR Measurement of Nonlocal Dispersion in Complex Flows. Physical Review Letters, 99(21):210602 1-4, November 2007.

[27] P. Mitra and P. Sen. Effects of microgeometry and surface relaxation on NMR pulsed-field-gradient experiments: Simple pore geometries. Physical Review B, 45(1):143-156, January 1992.

[28] F. Cros, J.-P. Korb, and L. Malier. Spectroscopic Mesopore Size Characterization and Diffusion Measurement in Closed Porosity by Xenon NMR. Langmuir, 16(26):10193-10197, December 2000.

[29] L. L. Latour, K. Svoboda, P. P. Mitra, and C. H. Sotak. Time-dependent diffusion of water in a biological model system. Proceedings of the National Academy of Sciences of the United States of America, 91(4):1229-33, February 1994.

[30] A.-M. Oros and N. J. Shah. Hyperpolarized xenon in NMR and MRI. Physics in Medicine and Biology, 49(20):R105-R153, October 2004.

[31] R. C. Wayne and R. M. Cotts. Nuclear-Magnetic-Resonance Study of Self-Diffusion in a Bounded Medium. Physical Review, 151(1):264-272, November 1966. 
[32] F. Laun, T. Kuder, W. Semmler, and B. Stieltjes. Determination of the Defining Boundary in Nuclear Magnetic Resonance Diffusion Experiments. Physical Review Letters, 107(4):2-5, July 2011.

[33] F. Laun, T. Kuder, A. Wetscherek, B. Stieltjes, and W. Semmler. NMR-based diffusion pore imaging. Physical Review E, 86(2):1-23, August 2012.

[34] P. P. Mitra and B. Pesaran. Analysis of dynamic brain imaging data. Biophysical Journal, 76(2):691-708, February 1999.

[35] C. J. Elkins and M. T. Alley. Magnetic resonance velocimetry: applications of magnetic resonance imaging in the measurement of fluid motion. Experiments in Fluids, 43(6):823-858, October 2007.

[36] R. Bandelli and K.R. Rajagopal. Start-up flows of second grade fluids in domains with one finite dimension. International Journal of Non-Linear Mechanics, 30(6):817-839, November 1995.

[37] N. B. Wood, S. J. Weston, P. J. Kilner, A. D. Gosman, and D. N. Firmin. Combined MR imaging and CFD simulation of flow in the human descending aorta. Journal of magnetic resonance imaging : JMRI, 13(5):699-713, May 2001.

[38] R. Codina. Stabilized finite element approximation of transient incompressible flows using orthogonal subscales. Computer Methods in Applied Mechanics and Engineering, 191(39-40):4295-4321, August 2002.

[39] C. Fetecau and C. Fetecau. Starting solutions for some unsteady unidirectional flows of a second grade fluid. International Journal of Engineering Science, 43(10):781789, June 2005.

[40] K. Kose. One-shot velocity mapping using multiple spin-echo EPI and its application to turbulent flow. Journal of Magnetic Resonance, 92:631-635, 1991.

[41] P. T. Callaghan, C. D. Eccles, and Y. Xia. NMR microscopy of dynamic displacements: k-space and q-space imaging. Journal of Physics E: Scientific Instruments, 820-822:1-4, 1988.

[42] B.J. Balcom, R.P. Macgregor, S.D. Beyea, D.P. Green, R.L. Armstrong, and T.W. Bremner. Single-Point Ramped Imaging withT1Enhancement (SPRITE). Journal of Magnetic Resonance, Series A, 123(1):131-134, November 1996. 
[43] P. Mansfield. Multi-planar image formation using NMR spin echoes. Journal of Physics C: Solid State Physics, 10(3):L55-L58, February 1977.

[44] C. B. Ahn, J. H. Kim, and Z. H. Cho. High-speed spiral-scan echo planar NMR imaging-I. IEEE Transactions on Medical Imaging, 5(1):2-7, January 1986.

[45] A. J. Sederman, M. D. Mantle, C. Buckley, and L. F. Gladden. MRI technique for measurement of velocity vectors, acceleration, and autocorrelation functions in turbulent flow. Journal of Magnetic Resonance, 166(2):182-189, February 2004.

[46] E.i Fukushima. Nuclear Magnetic Resonance as a Tool to Study Flow. Annual Review of Fluid Mechanics, 31:95-123, 1999.

[47] E. O. Stejskal and J. E. Tanner. Spin Diffusion Measurements: Spin Echoes in the Presence of a Time-Dependent Field Gradient. The Journal of Chemical Physics, 42(1):288, 1965.

[48] M. L. Johns. NMR studies of emulsions. Current Opinion in Colloid and Interface Science, 14(3):178-183, June 2009.

[49] M. L. Johns and K. G. Hollingsworth. Characterisation of Emulsion Systems Using NMR and MRI. Progress in Nuclear Magnetic Resonance Spectroscopy, 50(2-3):5170, 2007.

[50] J Karger and W Heink. The propagator representation of molecular transport in microporous crystallites. Journal of Magnetic Resonance, 51:1-7, 1983.

[51] P. N. Sen. Time-dependent diffusion coefficient as a probe of geometry. Concepts in Magnetic Resonance, 23A(1):1-21, September 2004.

[52] M. D. Hurlimann, K. G. Helmer, L. L. Latour, and C. H. Sotak. Restricted Diffusion in Sedimentary Rocks. Determination of Surface-Area-to-Volume Ratio and Surface Relaxivity. Journal of Magnetic Resonance, Series A, 111(2):169-178, December 1994.

[53] R. M. Cotts. Diffusion and diffraction. Nature, 351(6326):443-444, June 1991.

[54] P. T. Callaghan, A. Coy, and D. MacGowan. Diffraction-like effects in NMR diffusion studies of fluids in porous solids. Nature, 351:467-469, 1991.

[55] A. Coy and P. T. Callaghan. Pulsed Gradient Spin-Echo NMR" Diffusive Diffraction" Experiments on Water Surrounding Close-Packed Polymer Spheres. Journal of Colloid and Interface Science, 168:373-379, 1994. 
[56] J. Bear. Dynamics of Fluids in Porous Media. American Elsevier Publishing Company Inc., New York, 1972.

[57] F. A. L. Dullien. Porous Media. Fluid Transport and Pore Structure. Academic Press, New York, second edi edition, 1992.

[58] R. Brown. A Brief Account of the Microscopical Observations Made in the Months of June, July and August, 1827, on the Particles Contained in the Pollen of Plants; and on the General Existence of Active Molecules in Organic and Inorganic Bodies. Philosophical Magazine, 4:161-173, 1829.

[59] A. Einstein. The Motion of Elements Suspended in Static Liquids as Claimed in the Molecular Kinetic Theory of Heat. Annalen der Physik, 17:549-560, 1905.

[60] J. Perrin. The Origin of the Brownian Movement. Comptes rendus Hebdomadaires des Seances de l'Academie des Sciences, 147:530-532, 1908.

[61] J. Perrin. Brownian Motion and Molecular Reality. Annales de Chimie et de physique, 18:5-114, 1909.

[62] J. Perrin. Stokes Law and Brownian Motion. Comptes rendus Hebdomadaires des Seances de l'Academie des Sciences, 147:475-476, 1908.

[63] J. Perrin. Molecular Agitation and the Brownian Movement. Comptes rendus Hebdomadaires des Seances de l'Academie des Sciences, 146:967-970, 1908.

[64] A. Fick. Über Diffusion. Philosophical Magazine, 10:31-39, 1855.

[65] S. L. Codd, B. Manz, J. D. Seymour, and P. T. Callaghan. Taylor dispersion and molecular displacements in Poiseuille flow. Physical Review E, 60(4 Pt A):R3491-4, October 1999.

[66] P. T. Callghan. Translational Dynamics 8 Magnetic Resonance. Oxford University Press, New York, 1st edition, 2011.

[67] C. H. Neuman. Spin echo of spins diffusing in a bounded medium. The Journal of Chemical Physics, 60(11):4508, 1974.

[68] F. Bloch. Nuclear Induction. Physical Review, 70(7-8):460-474, October 1946.

[69] P. T. Callaghan. Principles of Nuclear Magnetic Resonance Microscopy. Clarendon Press, Oxford, New York, 1991. 
[70] A. Abragam. Principles of Nuclear Magnetism. Oxford University Press, New York, 1961.

[71] M. H. Levitt. Spin Dynamics: Basics of Nuclear Magnetic Resonance. Wiley, West Sussex, 2001.

[72] E. Hahn. Spin Echoes. Physical Review, 80(4):580-594, November 1950.

[73] H. Y. Carr and E. M. Purcell. Effects of Diffusion on Free Precession in Nuclear Magnetic Resonance Experiments. Physical Review, 94(3):630-638, May 1954.

[74] P. B. Kingsley. Methods of Measuring Spin-Lattice (T-1) Relaxation Times : An Annotated Bibliography. Concepts in Magnetic Resonance, 11(4):243-276, 1999.

[75] Bernhard Blümich. NMR Imaging of Materials. Clarendon Press, Oxford, New York, 2000.

[76] P. Mansfield and P. K. Grannell. NMR 'diffraction' in solids? Journal of Physics C: Solid State Physics, 6(22):L422-L426, November 1973.

[77] P. T. Callaghan, C. D. Eccles, and Y. Xia. NMR microscopy of dynamic displacements: k-space and q-space imaging. 820, 1988.

[78] P. J. Basser, J. Mattiello, and D. LeBihan. MR diffusion tensor spectroscopy and imaging. Biophysical Journal, 66(1):259-67, January 1994.

[79] Y. Shrot and L. Frydman. The effects of molecular diffusion in ultrafast twodimensional nuclear magnetic resonance. The Journal of Chemical Physics, 128(16):164513, April 2008.

[80] L. Frydman, T. Scherf, and A. Lupulescu. The acquisition of multidimensional NMR spectra within a single scan. Proceedings of the National Academy of Sciences of the United States of America, 99(25):15858-62, December 2002.

[81] Y. Shrot and L. Frydman. Single-scan 2D DOSY NMR spectroscopy. Journal of Magnetic Resonance, 195(2):226-31, December 2008.

[82] R. Freeman and E. Kupce. New methods for fast multidimensional NMR. Journal of Biomolecular NMR, 27(2):101-13, October 2003.

[83] M. J. Thrippleton, N. M. Loening, and J. Keeler. A fast method for the measurement of diffusion coefficients: one-dimensional DOSY. Magnetic Resonance in Chemistry, 41(6):441-447, June 2003. 
[84] N. M. Loening, J. Keeler, and G. A. Morris. One-dimensional DOSY. Journal of Magnetic Resonance, 153(1):103-12, November 2001.

[85] P. Gelderen, A. Olson, and C. Moonen. A Single-Shot Diffusion Experiment. Journal of Magnetic Resonance, Series A, 103:105-108, 1993.

[86] Y.-Q. Song and X. Tang. A one-shot method for measurement of diffusion. Journal of Magnetic Resonance, 170(1):136-48, September 2004.

[87] S. J. Doran and M. Décorps. A robust, single-shot method for measuring diffusion coefficients using the "Burst" sequence. Journal of Magnetic Resonance, Series A, 117:311-316, 1995.

[88] S. Peled, C. H. Tseng, A. A. Sodickson, R. W. Mair, R. L. Walsworth, and D. G. Cory. Single-shot diffusion measurement in laser-polarized Gas. Journal of Magnetic Resonance, 140(2):320-4, October 1999.

[89] L. Li and C. H. Sotak. Diffusion measurements by pulsed field-gradient multiple spin echoes. Journal of Magnetic Resonance (1969), 92(2):411-420, April 1991.

[90] J. P. Stamps, B. Ottink, J. M. Visser, J. P. van Duynhoven, and R. Hulst. Difftrain: a novel approach to a true spectroscopic single-scan diffusion measurement. Journal of Magnetic Resonance, 151(1):28-31, July 2001.

[91] W. S. Price and P. W. Kuchel. Effect of nonrectangular field gradient pulses in the stejskal and tanner (diffusion) pulse sequence. Journal of Magnetic Resonance (1969), 94(1):133-139, August 1991.

[92] J. Jovicich, S. Czanner, D. Greve, and et al. Reliability in multi-site structural MRI studies: effects of gradient non-linearity correction on phantom and human data. NeuroImage, 30(2):436-43, May 2006.

[93] A. Janke, H.i Zhao, G. J. Cowin, G. J. Galloway, and D. M. Doddrell. Use of spherical harmonic deconvolution methods to compensate for nonlinear gradient effects on MRI images. Magnetic resonance in medicine : official journal of the Society of Magnetic Resonance in Medicine / Society of Magnetic Resonance in Medicine, 52(1):115-22, July 2004.

[94] C.-M. Lai. Reconstructing NMR images under nonlinear field gradients. Journal of Physics E: Scientific Instruments, 16(1):34-38, January 1983. 
[95] S. Langlois, M. Desvignes, J. M. Constans, and M. Revenu. MRI geometric distortion: a simple approach to correcting the effects of non-linear gradient fields. Journal of Magnetic Resonance Imaging, 9(6):821-31, June 1999.

[96] R. Bammer, M. Markl, A. Barnett, B. Acar, M. T. Alley, N. J. Pelc, G. H. Glover, and M. E. Moseley. Analysis and generalized correction of the effect of spatial gradient field distortions in diffusion-weighted imaging. Magnetic Resonance in Medicine, 50(3):560-9, September 2003.

[97] P. Bendel. Spin-echo attenuation by diffusion in nonuniform field gradients. Journal of Magnetic Resonance, 86:509-515, 1990.

[98] P. T. Callaghan and J. Stepisnik. Spatially-distributed pulsed gradient spin echo NMR using single-wire proximity. Physical Review Lettersetters, 75(24), 1995.

[99] J. P. Stockmann, P. A. Ciris, G. Galiana, L. Tam, and R. T. Constable. O-space imaging: Highly efficient parallel imaging using second-order nonlinear fields as encoding gradients with no phase encoding. Magnetic Resonance in Medicine, 64(2):447-56, August 2010.

[100] J. P. Stockmann, G. Galiana, L. Tam, T. Nixon, and R. T. Constable. First OSpace images using a high-power, actively-shielded, 12-cm Z2 gradient insert on a human 3T scanner. In 19th Annual Proceedings of the International Society for Magnetic Resonance in Medicine, volume 19, page 717, Montreal, 2011.

[101] W. C. Kittler, P. Galvosas, and M. W. Hunter. Parallel acquisition of q-space using second order magnetic fields for single-shot diffusion measurements. Journal of Magnetic Resonance, 244C:46-52, April 2014.

[102] O. Posnansky, R. Huang, and N. J. Shah. Translational free random walk of spins in the presence of a parabolic magnetic field. Journal of Magnetic Resonance, 173(1):1-9, March 2005.

[103] P. Le Doussal and P. N. Sen. Decay of nuclear magnetization by diffusion in a parabolic magnetic field: An exactly solvable model. Physical Review B, 46(6), 1992.

[104] R. M. Cotts, M. J. R. Hoch, T. Sun, and J. T. I. Markert. Pulsed Field Gradient Stimulated Echo Methods for Improved NMR Diffusion Measurements in Heterogeneous Systems. Journal of Magnetic Resonance, 83(2):252-266, 1989. 
[105] J. E. Tanner. Use of the Stimulated Echo in NMR Diffusion Studies. The Journal of Chemical Physics, 52(5):2523, 1970.

[106] L. F. Gladden and A. J. Sederman. Recent advances in flow MRI. Journal of Magnetic Resonance, 229:2-11, April 2013.

[107] C. J. Davies, A. J. Sederman, C. J. Pipe, G. H. McKinley, L. F. Gladden, and M. L. Johns. Rapid measurement of transient velocity evolution using GERVAIS. Journal of Magnetic Resonance, 202(1):93-101, January 2010.

[108] M. W. Garrett. Axially Symmetric Systems for Generating and Measuring Magnetic Fields. Part I. Journal of Applied Physics, 22(9):1091, 1951.

[109] M. J. E. Golay. Field Homogenizing Coils for Nuclear Spin Resonance Instrumentation. Review of Scientific Instruments, 29(4):313, 1958.

[110] W. A. Anderson. Electrical Current Shims for Correcting Magnetic Fields. Review of Scientific Instruments, 32(3):241, 1961.

[111] D. G. Norris and J. M. S. Hutchison. Concomitant magnetic field gradients and their effects on imaging at low magnetic field strengths. Magnetic Resonance Imaging, 8(1):33-37, January 1990.

[112] D. A. Yablonskiy, A. L. Sukstanskii, and J. J. H. Ackerman. Image artifacts in very low magnetic field MRI: The role of concomitant gradients. Journal of Magnetic Resonance, 174(2):279-286, June 2005.

[113] T. R. Brown, B. M. Kincaid, and K. Ugurbil. NMR chemical shift imaging in three dimensions. Proceedings of the National Academy of Sciences of the United States of America, 79(11):3523-6, June 1982.

[114] A. A. Maudsley, H. E. Simon, and S. K. Hilal. Magnetic field measurement by NMR imaging. Journal of Physics E: Scientific Instruments, 17(3):216-220, March 1984.

[115] M. Holz, S. R. Heil, and A. Sacco. Temperature-dependent self-diffusion coefficients of water and six selected molecular liquids for calibration in accurate $1 \mathrm{H}$ NMR PFG measurements. Physical Chemistry Chemical Physics, 2(20):4740-4742, 2000.

[116] D. Johnson, T. Plona, and C. Scala. Tortuosity and Acoustic Slow Waves. Physical Review Letters, 49(25):1840-1844, 1982. 
[117] P. T. Callaghan, S. L. Codd, and J. D. Seymour. Phenomena Arising from Translational Spin Motion in Gradient Spin Echo. Concepts in Magnetic Resonance, 11(4):181-202, 1999.

[118] M. W. Hunter, A. N. Jackson, and P. T. Callaghan. Nuclear magnetic resonance measurement and lattice-Boltzmann simulation of the nonlocal dispersion tensor. Physics of Fluids, 22(2):027101, 2010.

[119] M. W. Hunter, A. N. Jackson, and P. T. Callaghan. PGSE NMR measurement of the non-local dispersion tensor for flow in porous media. Journal of Magnetic Resonance, 204(1):11-20, May 2010. 